\title{
Dynamics of NorTh AtlantiC Western Boundary CURREnTs
}

\author{
by \\ Isabela Astiz Le Bras \\ B.A., University of California, Berkeley (2010) \\ Submitted in partial fulfillment of the requirements for the degree of \\ Doctor of Philosophy \\ at the \\ MASSACHUSETTS INSTITUTE OF TECHNOLOGY \\ and the \\ WOODS HOLE OCEANOGRAPHIC INSTITUTION
}

February 2017

(C)2017 Isabela Astiz Le Bras. All rights reserved.

The author hereby grants to MIT and WHOI permission to reproduce and to distribute publicly paper and electronic copies of this thesis document in whole or in part in any medium now known or hereafter created.

Author

Joint Program in Physical Oceanography Massachusetts Institute of Technology \& Woods Hole Oceanographic Institution

December 23, 2016

Certified by

John M. Toole

Senior Scientist in Physical Oceanography

Woods Hole Oceanographic Institution

Thesis Supervisor

Accepted by

Lawrence J. Pratt

Chair, Joint Committee for Physical Oceanography Massachusetts Institute of Technology Woods Hole Oceanographic Institution 


\title{
Dynamics of NorTh Atlantic \\ Western Boundary CURRENTS
}

by

Isabela Astiz Le Bras

Submitted to the MIT-WHOI Joint Program in Physical Oceanography on December 23, 2016, in partial fulfillment of the requirements for the degree of Doctor of Philosophy in Physical

Oceanography

\begin{abstract}
The Gulf Stream and Deep Western Boundary Current (DWBC) shape the distribution of heat and carbon in the North Atlantic, with consequences for global climate. This thesis employs a combination of theory, observations and models to probe the dynamics of these two western boundary currents.

First, to diagnose the dynamical balance of the Gulf Stream, a depth-averaged vorticity budget framework is developed. This framework is applied to observations and a state estimate in the subtropical North Atlantic. Budget terms indicate a primary balance of vorticity between wind stress forcing and dissipation, and that the Gulf Stream has a significant inertial component.
\end{abstract}

The next chapter weighs in on an ongoing debate over how the deep ocean is filled with water from high latitude sources. Measurements of the DWBC at Line W, on the continental slope southeast of New England, reveal water mass changes that are consistent with changes in the Labrador Sea, one of the sources of deep water thousands of kilometers upstream. Coherent patterns of change are also found along the path of the DWBC. These changes are consistent with an advective-diffusive model, which is used to quantify transit time distributions between the Labrador Sea and Line W. Advection and stirring are both found to play leading order roles in the propagation of water mass anomalies in the DWBC.

The final study brings the two currents together in a quasi-geostrophic process model, focusing on the interaction between the Gulf Stream's northern recirculation gyre and the continental slope along which the DWBC travels. We demonstrate that the continental slope restricts the extent of the recirculation gyre and alters its forcing mechanisms. The recirculation gyre can also merge with the DWBC at depth, and its adjustment is associated with eddy fluxes that stir the DWBC with the interior. This thesis provides a quantitative description of the structure of the overturning circulation in the western North Atlantic, which is an important step towards understanding its role in the climate system.

Thesis Supervisor: John M. Toole

Title: Senior Scientist in Physical Oceanography

Woods Hole Oceanographic Institution 


\section{ACKNOWLEDGMENTS}

First and foremost, I thank my advisor, John Toole. John gave me the freedom to explore my own ideas, guided me with his thought-provoking questions, and was always available for discussions. I strive to emulate John's careful and creative approach to science as well as his kindness. I am ever thankful for the support and faith he showed me throughout my thesis and look forward to our continued collaborations.

My committee was a great help and a pleasure to interact with. I thank Amy Bower for many exciting conversations about the North Atlantic, and for reminding me to keep my language precise. Glenn Flierl offered many deep insights as my work developed, and his dedication to science and students is inspiring. Steve Jayne provided invaluable assistance with the QG model he started writing as a WHOI Summer Student Fellow, and found time to meet and help me keep perspective, even during hurricane season. Mike Spall's thoughtful questions and suggestions have shaped this thesis and helped me learn to identify interesting and tractable scientific problems. Finally, I thank Young-Oh Kwon for chairing my defense, for access to his computer server and his enthusiastic teaching.

The WHOI PO department and MIT EAPS departments provided stimulating research environments over the last 5 years. Magdalena Andres and Jake Gebbie have been excellent mentors and I have benefitted enormously from conversations and classes with Joe Pedlosky, Ken Brink, Amala Mahadevan, Terry Joyce, Susan Lozier, Stephanie Waterman, and John Marshall. Thanks are also due to Patrick Heimbach, Chris Hill and Diana Lees Spiegel for providing the ECCO state estimate fields analyzed in Chapter 2.

I am grateful for the opportunities I had to go to sea with John Toole, Ruth Curry, Magdalena Andres, and Leah Trafford. The Line W observations presented in Chapter 3 are the result of many years of hard work by many people, including Michael McCartney, Terry Joyce, Dan Torres, Scott Worrilow, Brian Hogue, the WHOI mooring group, and the crews of the Knorr and Oceanus.

I was also fortunate enough to visit England and Germany over the course of my Ph.D. and form connections with scientists at Southhampton, Kiel, Hamburg and Bremen. In particular, I thank Martin Visbeck, Jürgen Fischer and Patricia Handmann for hosting my visit to Kiel. I thank Igor Yashayaev for providing the Labrador Sea data used in Chapter 3 , and many interesting email conversations.

I have had a lot of fun in the Joint Program. My PO peers, Becca Jackson, Joern Callies, Alec Bogdanoff and Deepak Cherian provided many interesting scientific discussions, laughs and support. My roommates, Julie van der Hoop and Sophie Chu were like sisters to me,

and the many other houses of friends in Woods Hole, at Hinckley, Fern, and Huettner, as well as the far reaches of Falmouth and Cambridge made the long winters full of potluck-fun. 
I also wish to thank Harriet for her love and encouragement, and for making Figure 4-10. I look forward to many years full of science, cooking and windsurfing together.

Finally, I thank my family. In particular, I thank my brothers and my parents, Luciana Astiz and Ronan Le Bras, for their tireless support and for inspiring me to pursue a career in science. Gracias mama and merci papa!

My research was funded by National Science Foundation grants OCE-0241354, OCE0726720 and OCE-1332667 as well as a graduate fellowship from the American Meteorological Society. Support for travel and educational supplies was also provided by the MIT Houghton Fund and the WHOI Academic Programs Office. Special thanks are due to APO and administrators at MIT and the WHOI PO department for running the Joint Program seemlessly. 


\section{Contents}

1 Introduction $\quad 15$

1.1 Historical background $\ldots \ldots \ldots \ldots \ldots$

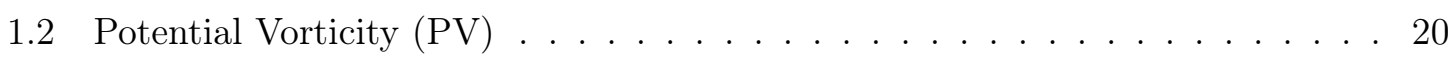

1.3 Scientific context . . . . . . . . . . . . . . . . . . 22

1.4 Thesis Overview . . . . . . . . . . . . . . . . . . . 25

2 A Vorticity Budget for the Western North Atlantic Based on Observations 27

2.1 Abstract . . . . . . . . . . . . . . . . . . . . . 28

2.2 Introduction . . . . . . . . . . . . . . . . . . . . . 29

2.3 Mathematical framework . . . . . . . . . . . . . . . . . . . . 33

2.4 Wind Stress Forcing . . . . . . . . . . . . . . . . . . . . . . . . 42

2.5 Advective vorticity flux . . . . . . . . . . . . . . . . . . 46

2.6 Remaining budget terms . . . . . . . . . . . . . . . 54

2.7 Conclusions . . . . . . . . . . . . . . . . . . . 56

3 Water Mass Properties in the Deep Western Boundary Current $\quad 61$

3.1 Abstract . . . . . . . . . . . . . . . . . . . . . 62

3.2 Introduction . . . . . . . . . . . . . . . . . 63

3.3 Datasets . . . . . . . . . . . . . . . . . . . 68

3.3 .1 Line $\mathrm{W} \ldots \ldots \ldots \ldots \ldots$

3.3 .2 Central Labrador Sea . . . . . . . . . . . . . . . . . . 69

3.4 Line $\mathrm{W}$ mooring $\theta-S$ shifts . . . . . . . . . . . . . . . . . . . . 70

3.5 Coherence between hydrographic anomalies in neutral density space . . . . . . 74

3.6 Water mass class averages . . . . . . . . . . . . . . . . . . 80

3.7 Quantification of water mass transit time distributions . . . . . . . . . . 87

3.7.1 Introduction to model framework . . . . . . . . . . . . . . 87

3.7 .2 Analytical model . . . . . . . . . . . . . . . . . . 88

3.7 .3 Forward model . . . . . . . . . . . . . . . . . . . . . . . . 98

3.8 Conclusions . . . . . . . . . . . . . . . . . . . . . . . . . 101

4 A Model of the Interaction Between the Gulf Stream Northern Recirculation Gyre and the Deep Western Boundary Current 107

4.1 Abstract . . . . . . . . . . . . . . . . . . . . . . . 108 
4.2 Introduction . . . . . . . . . . . . . . . . . . . . 109

4.3 Model setup . . . . . . . . . . . . . . . . . . . . 111

4.4 Jet evolution . . . . . . . . . . . . . . . . . . . . . . . . . . . . . 119

4.5 The meridional extent of recirculation gyres . . . . . . . . . . . . 120

4.6 PV budget analysis . . . . . . . . . . . . . . . . . . . . . . 124

4.7 Varying model parameters . . . . . . . . . . . . . . . . . . . . . 129

4.7 .1 Varying jet instability . . . . . . . . . . . . . . 136

4.7.2 Varying the distance between the jet and the slope . . . . . . . . . . 140

4.8 Discussion . . . . . . . . . . . . . . . . . . . . . . . . . . . . . . . . 142

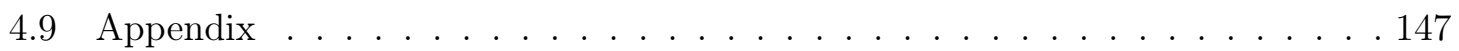

4.9 .1 Numerical method . . . . . . . . . . . . . . . . . . . . . . . . 147

4.9.2 Differences with Waterman and Jayne (2011) model setup . . . . . . . 147

4.9.3 Sensitivities to fixed model parameters . . . . . . . . . . . . . . . . 148

4.9.4 Apparent interference from the southern sponge . . . . . . . . . . . . 149

4.9.5 Estimating the time rate of change of $q_{2} \ldots \ldots \ldots \ldots$

5 Conclusions $\quad 157$

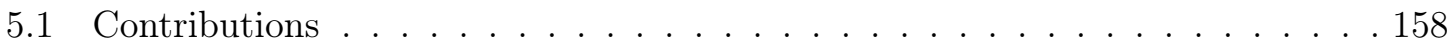

5.2 Implications and Outlook . . . . . . . . . . . . . . . 160 


\section{List OF Figures}

1-1 Chart of the Gulf Stream by Benjamin Franklin. . . . . . . . . . . . . 17

1-2 Oxygen concentrations from 2000-3000 m measured on the 1925-1927 Meteor

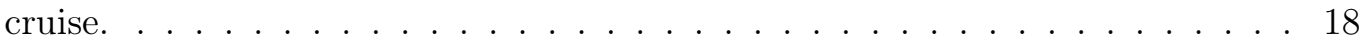

1-3 A schematic of Atlantic circulation from Stommel (1957). . . . . . . . . . . 19

2-1 Map of WOCE/CLIVAR A22 cruise tracks and the ECCO boundary. . . . . . 32

2-2 Depth of the study volume in the Gouretski and Koltermann (2004) climatology and ECCO state estimate. . . . . . . . . . . . . . . . . . 34

2-3 Average wind stress in the regions of interest from four different products. . . 41

2-4 Time series of wind stress forcing term from satellite data products. . . . . . . 43

2-5 Time series of the wind stress forcing and vorticity flux terms in the ECCO model. . . . . . . . . . . . . . . . . . . . . . . . . 44

2-6 Components of calculation of vorticity flux across the A22 cruise track . . . . 48

2-7 As in Figure 2-6 for the ECCO state estimate analysis. . . . . . . . . . . . . . 52

2-8 Time series of the remaining significant terms in the vorticity budget and demonstration of approximate balance in the ECCO model. . . . . . . . . . . 53

2-9 Maps showing the relationship of $\mathrm{f} / \mathrm{h}$ contours, time mean depth-averaged flow and lateral friction for the study volume in the ECCO model. . . . . . . 57

3-1 Map of dataset locations along the path of the Deep Western Boundary Current 64

3-2 Time evolution of a) potential temperature and b) salinity profiles in the central Labrador Sea. . . . . . . . . . . . . . . . . . . . . . . 66

3-3 Map of the Labrador Sea highlighting observation locations used to form the annual average central Labrador Sea profiles. . . . . . . . . . . . . . . . 70

3-4 $\theta-S$ properties in the central Labrador Sea, at Line W moorings and at BATS. 71

3-5 Evolution of $\theta-S$ properties at Line $\mathrm{W}$ moored array from 2005 to 2013 . . . . 73

3-6 Water mass property anomaly time series and mean profiles in the central Labrador Sea and at Line $\mathrm{W}$ in neutral density space. . . . . . . . . . . . . 76

3-7 Results from lagged correlation analysis between Labrador Sea and Line W layer thickness, potential temperature and salinity anomalies within neutral density bins. . . . . . . . . . . . . . . . . . . . . . 79

3-8 Mean salinity within water masses for datasets along the path of the DWBC. 82

3-9 Mean salinity within water masses measured by Line $\mathrm{W}$ moorings. . . . . . . 83 
3-10 As in Figure 3-8 but for water mass layer thickness. . . . . . . . . . . . . . 85

3-11 As in Figure 3-9 but water mass layer thickness as measured by Line W moorings. . . . . . . . . . . . . . . . . . 86

3-12 Transit time distributions and sample sinusoidal signals for different limits of the Waugh and Hall (2005) model. . . . . . . . . . . . . . . . . . . . 91

3-13 Sinusoidal fits to central Labrador Sea and Line W mean dLSW and NEADW potential temperature, salinity and layer thickness. . . . . . . . . . . . . . . . 92

3-14 Parameter range of sinusoidal fits to Labrador Sea and Line W potential temperature and salinity data shown in relative amplitude and phase lag space. 93

3-15 Solutions to parameter space sensitivity analysis. . . . . . . . . . . . . . 96

3-16 Range of potential transit time distributions solutions from the Labrador Sea to Line $\mathrm{W}$ for dLSW. . . . . . . . . . . . . . . . . . . . . . . 97

3-17 Normalized cost functions for forward model fits to Line W dLSW potential temperature, salinity and layer thickness time series. . . . . . . . . . . . . 99

3-18 Best fits from forward model to Line W dLSW potential temperature, salinity and layer thickness anomaly times series. . . . . . . . . . . . . . . . 100

4-1 Schematic of the barotropic circulation in the western North Atlantic from Zhang and Vallis (2007), adapted from Hogg (1992). . . . . . . . . . . . . 110

4-2 Meridional structure of the streamfunction, velocity, potential vorticity and meridional potential vorticity gradient at the model western boundary. . . . . 114

4-3 Mean streamfunction $(\bar{\psi})$ and PV $(\bar{q})$ in the upper and lower layers for the basic model setup. . . . . . . . . . . . . . . . . . . . . . . . . . . 115

4-4 Steepness of bathymetry along the continental slope, and comparison of Line W and model bathymetry and steepness. . . . . . . . . . . . . . . . . 118

4-5 Normalized meridional PV gradients and meridional eddy PV fluxes for averages in sections of the recirculation gyre depicted in Figure 4-3 . . . . . . . . 120

4-6 Time-mean profiles of lower layer PV, $\overline{q_{2}}$, relative vorticity, $\overline{\zeta_{2}}$, thickness, $\left(\overline{\psi_{1}}-\overline{\psi_{2}}\right) / S_{2}$, and zonal velocity, $\overline{u_{2}}$, at the zonal position of jet stabilization,

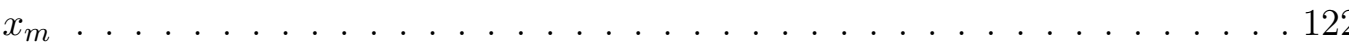

4-7 Analytic recirculation gyre meridional extent prediction plotted against measured meridional extent in the QG model (both in $\mathrm{km}$ ) for model configurations with different inflowing jet velocities. . . . . . . . . . . . . . . . 123

4-8 Time-mean zonal velocity profiles in the upper model layer at the zonal position of jet stabilization. . . . . . . . . . . . . . . . . . . . . . . . . . . . . 124

4-9 Profiles of meridional eddy PV flux integrated zonally across the recirculation gyre for model configurations without a slope or DWBC (blue), with a slope, but no DWBC (green), and with both a slope and DWBC (red) . . . . . . . 125

4-10 Schematic illustration of the system PV budget in the model lower layer. . . . 126 
4-11 Cumulative eddy and mean PV flux convergences in the lower layer, integrated northward from the jet axis, $y_{j} \ldots \ldots \ldots$. . . . . . . . . . . 127

4-12 PV budget synthesis for model configurations without a slope or DWBC, with a slope, but no DWBC, and both a slope and DWBC. . . . . . . . . . . . 128

4-13 The time mean streamfunction in the lower layer, $\overline{\psi_{2}}$, for model configurations with varying initial jet strength and proximity to bathymetric slope. . . . . . 132

4-14 Lower layer meridional eddy PV fluxes integrated zonally along the jet axis for model configurations shown in Figure 4-13, with an explanatory schematic.133

4-15 Upper layer PV $\left(q_{1}\right)$ snapshots for the model configurations shown in Figures

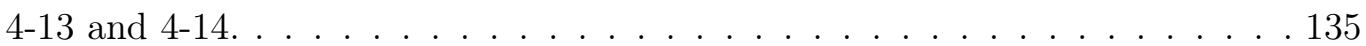

4-16 Growth rate as a function of zonal wavenumber for the model configurations shown in Figures 4-13-4-15 for the inflowing jet profile, and the time mean profiles at 1000 and $1600 \mathrm{~km}$ downstream. . . . . . . . . . . . . 136

4-17 As in Figure 4-6, for the model configurations with varying inflowing jet velocities, and hence jet instability. . . . . . . . . . . . . . 137

4-18 Analytic meridional extent prediction against meridional extent in the QG model (both in $\mathrm{km}$ ) for model configurations with varying jet velocities, with a slope and DWBC. . . . . . . . . . . . . . . . . . . . 138

4-19 PV budget synthesis for model configurations with varying inflowing jet velocities, and hence jet instability. . . . . . . . . . . . . . . . . . 138

4-20 Recirculation gyre and DWBC transports for model configurations with varying inflowing jet velocities, and hence jet instability. . . . . . . . . . . . . . 139

4-21 As in Figure 4-6, for model configurations with different distances between the unstable jet and DWBC. . . . . . . . . . . . . . . . . . . . . . 141

4-22 PV budget synthesis for model configurations with different distances between the unstable jet and DWBC. . . . . . . . . . . . . . . . . . . . . 142

4-23 Recirculation gyre and DWBC transports for model configurations with different distances between the unstable jet and DWBC. . . . . . . . . . . . . . 143

4-24 Nondimensional value of sponge region linear friction coefficient, $R_{\text {sponge }}$ in the model domain. . . . . . . . . . . . . . . . . . . . . . . . . . 147

4-25 Mean lower layer streamfunction, $\overline{\psi_{2}}$, for different values of the viscosity coefficient, A . . . . . . . . . . . . . . . . . . . . . 149

4-26 Meridional profiles of integrate meridional eddy PV flux, and time series of domain integrated enstrophy and psi variance for model runs in which a slope and DWBC are added sequentially. . . . . . . . . . . . . . . . . . . 151

4-27 As in Figure 4-26, but for model configurations with different inflowing jet velocities. . . . . . . . . . . . . . . . . . . . . . . . . . 152 
4-28 Time series of domain integrated enstrophy and $\psi$ for model configurations with inflowing jet velocity of $0.63 \mathrm{~ms}^{-1}$ and $1.44 \mathrm{~m} \mathrm{~s}^{-1}$. . . . . . . . . . . 153

4-29 As in Figure 4-26, but for model configurations with different distances between the jet and the center of the bathymetric slope. . . . . . . . . . . . . 154

4-30 Estimates of $\iint\left(\overline{d q_{2} / d t}\right) d A$ during three different time periods, for base case runs in which a slope and DWBC are added sequentially. . . . . . . . . . . 155

4-31 As in Figure 4-30 but for model configurations with different inflowing jet velocities. . . . . . . . . . . . . . . . . . . . . 156

4-32 As in Figure 4-30 but for model configurations with different distances between the jet and the center of the bathymetric slope. . . . . . . . . . . . . 156 


\section{LIST OF TABLES}

2-1 Summary of wind stress forcing and advective vorticity flux budget terms in both observations and in the ECCO state estimate. . . . . . . . . . . . . . . 46

2-2 Summary of all time-mean vorticity budget terms in the ECCO state estimate and their mean/eddy breakdown. . . . . . . . . . . . . . . . . 56

3-1 Water mass definitions. . . . . . . . . . . . . . . . . 67

3-2 Trends in dLSW water mass properties measured by Line W moorings. . . . . 87

3-3 Parameters of sinuisoidal fits to mean dLSW properties and resulting solutions. 90

3-4 As in table $3-3$ but for NEADW. . . . . . . . . . . . . . . . . . . . . 94

4-1 Dimensionalization of variables f . . . . . . . . . . . . . . . . . 113 
Chapter 1

\section{INTRODUCTION}


In the North Atlantic, two large-scale ocean circulation patterns redistribute heat, salt and carbon, with significant consequences for marine ecosystems (Schmittner, 2005), global sea level (Vellinga and Wood, 2008) and climate (Wunsch, 2005; Kwon et al., 2010): the winddriven and overturning circulations. The wind-driven circulation is forced by the prevailing global wind belts, the easterlies, westerlies and trade winds, forming a subtropical and subpolar gyre in the North Atlantic, each thousands of kilometers wide. The overturning circulation in the North Atlantic is composed primarily of cold water which sinks at high northern latitudes and travels toward the other pole at depth over tens of thousands of kilometers, and a warmer return flow at the surface.

Both of these circulation patterns include relatively strong and narrow $(O 100 \mathrm{~km})$ currents confined to the basin's western boundary, termed western boundary currents. This thesis is focused on the western boundary current of the North Atlantic subtropical gyre, the Gulf Stream, and the western boundary current of the North Atlantic overturning circulation, the Deep Western Boundary Current (DWBC).

Using a combination of observations and models, in this thesis I investigate the dynamics of the forcing of the Gulf Stream, the propagation of water mass properties in the DWBC and the interaction between the two currents. In this chapter, I will provide background and motivation to contextualize this thesis, as well as a road map for the remaining thesis chapters.

\subsection{Historical background}

Because of its significant impact on navigation in the western North Atlantic, seafarers have had a sense of the Gulf Stream since the 16th century (MacLeish, 1989). It was famously charted by Benjamin Franklin, who acknowledges whaling Captain Folger for informing him of the course, strength and extent of the stream (see Figure 1-1).

The deep ocean circulation was more elusive. In an essay at turn of the 19th century, Rumford (1800), showed an early appreciation for the high-latitude source of the cold water below the thermocline throughout the world ocean. Yet, as Deacon (1954) wrote, "We have known for 150 years that there is water, nearly ice-cold, at the bottom of the tropical Atlantic Ocean, and that it must flow there from the Antarctic, but we can still argue whether it took 18 years or 1800, and there is as much uncertainty about the forces which move it there." With the Meteor Expedition, from 1925 to 1927, came the first indication that the equatorward spreading of high-latitude water was western intensified. As shown in Figure 12, they measured a core of high oxygen water on the west of the Atlantic basin at 2000-3000 m depth (Wüst, 1935).

The basic mathematical theory governing both of these large-scale circulation patterns was proposed by Henry Stommel in the 1940's and 50's (Stommel, 1948; Stommel et al., 


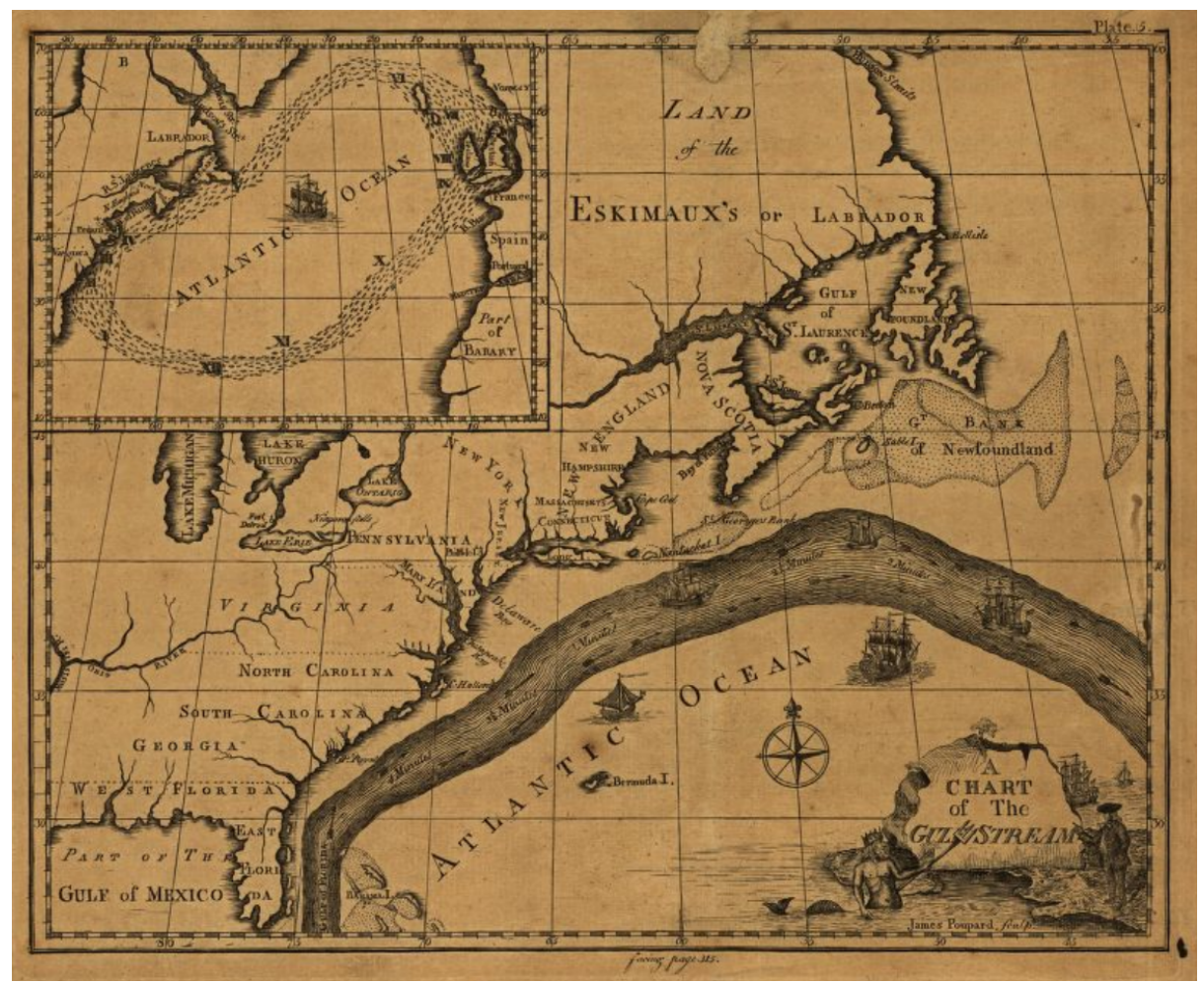

Figure 1-1: Chart of the Gulf Stream by Benjamin Franklin. Appeared in Transactions of the American Philosophical Society, 1786. Image courtesy of the Library of Congress, mounted on cloth.

1958). Our basic understanding of the circulation in the North Atlantic continues to be shaped by his insights, and his schematic of the superposition of the wind-driven and overturning circulations in Atlantic, Figure 1-3, remains relevant to our current understanding of the large-scale Atlantic circulation (Richardson, 2008). At the heart of both of these theories is the significance of planetary rotation on the large-scale movement of fluids, which can be conveniently framed in terms of Potential Vorticity (PV). 


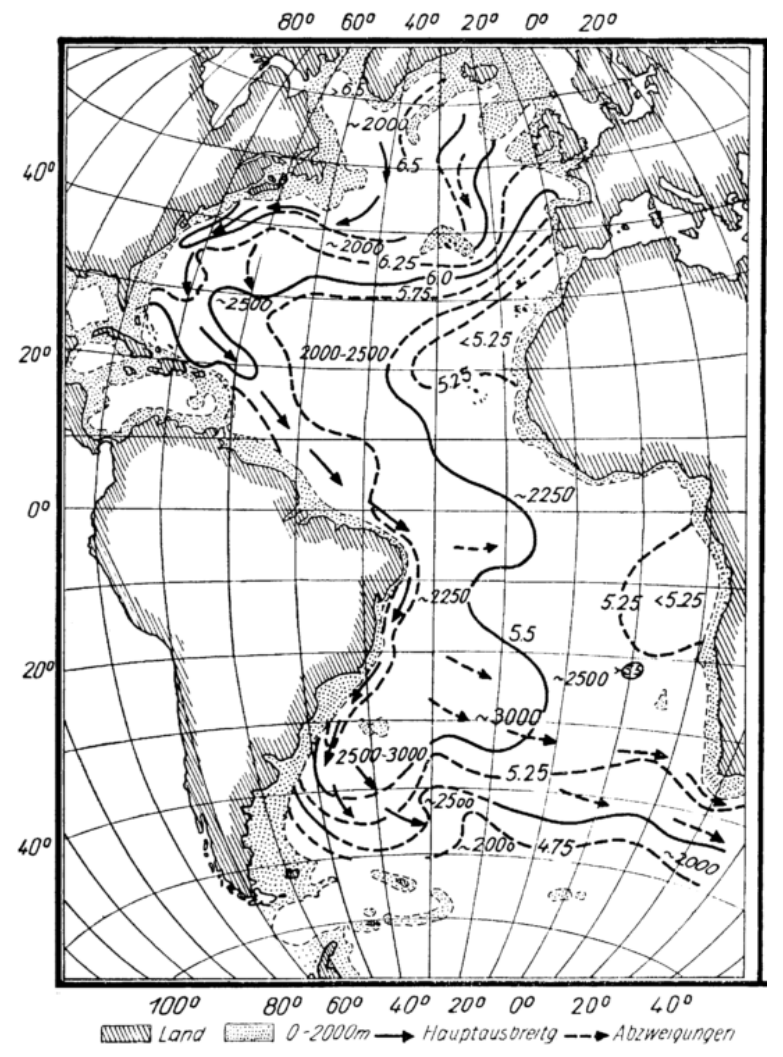

Figure 1-2: Oxygen concentrations from 2000-3000 m measured on the 1925-1927 Meteor cruise. Image from Richardson (2008). 

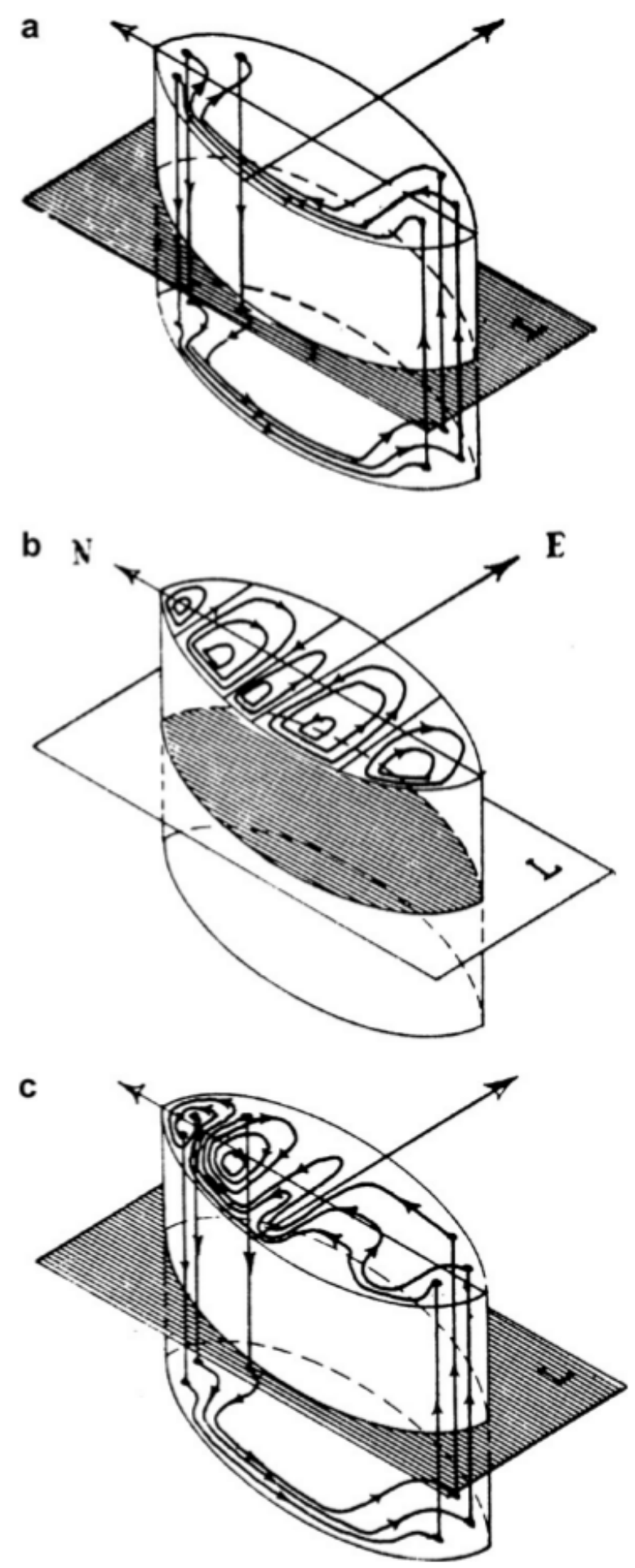

Figure 1-3: A schematic of Atlantic circulation from Stommel (1957). His caption: A schematic interpretation of the circulation in the Atlantic Ocean constructed by superposition of an internal model associated with flow across a level surface L at mid-depth (a) and a purely wind-driven circulation in the surface layers (b). The sum of these two is shown in figure (c). 


\section{$1.2 \quad$ Potential Vorticity (PV)}

PV is a quantity with enormous utility in physical oceanography. It is a scalar field that emerges when the momentum and buoyancy equations are combined and sheds light on the essential forcing mechanisms that control the circulation. Because it features in each of the main thesis chapters, in this section I will describe its derivation and highlight the different forms of PV that will appear throughout.

The dynamics considered in this thesis are in the low Rossby number limit. The Rossby number describes the relative magnitude of advective and Coriolis terms in the momentum equation and hence the importance of rotation. The Rossby number is

$$
R o=\frac{U}{f L}
$$

where $U$ is a characteristic velocity scale, $L$ a characteristic length scale and $f$ is the Coriolis parameter, which is twice the frequency of the earth's rotation multiplied by the sine of the latitude, to project the vector of the earth's rotation onto the vertical direction of the local coordinate frame. A low Rossby number means that the frequency of the described motions is smaller than the frequency of rotation in the local coordinate frame, which can mean that the described motions are slow or occur over large scales. In general, this corresponds to motions in the mid- and high-latitude ocean that occur over time scales longer than weeks and spatial scales larger than hundreds of kilometers.

In the low Rossby number limit, the primary balance of horizontal momentum reduces to the geostrophic balance:

$$
f \times \mathbf{u}=-\frac{\nabla p}{\rho_{0}}
$$

where $\mathbf{u}$ is the horizontal velocity vector, $p$ is pressure, $\rho_{0}$ is a reference density and cross products and gradients are in the horizontal plane. In geostrophic balance, flow follows contours of pressure. This principle enables the use of sea surface height to infer flow patterns, for example.

By taking the curl of the horizontal momentum equations to form a vorticity equation, this first order steady state balance is eliminated, as the curl of a gradient is zero. This draws the smaller terms in the momentum equation to the forefront, such as forcing and dissipation. Though the dominant balance can be eliminated through the vorticity equation to gain insight into forcing mechanisms, it can be difficult to interpret the vorticity equation as it describes a vector field. To overcome this, the vorticity equation can be spatially integrated to form an equation for a scalar field, the circulation. In Chapter 2, we construct a circulation equation and diagnose the size of its terms to gain insight into the large-scale vorticity balance of the Gulf Stream. 
However, the terms in a circulation equation depend on the integration volume. The PV equation, which emerges from the combination of the momentum and buoyancy equations, is more general because it describes a full scalar field. Holland et al. (1984) summed up the power of PV effectively in their statement, "A theory of the scalar fields q (PV) and $\rho$ (density) is tantamount to a theory of the general circulation."

The most general form of PV is due to Ertel (1942),

$$
q=\frac{2 \boldsymbol{\Omega}+\boldsymbol{\omega}}{\rho} \cdot \nabla \rho
$$

where $\boldsymbol{\Omega}$ is the earth's rotation vector and $\omega=\nabla \times \mathbf{u}$ is the vorticity of the flow, referred to as the relative vorticity; where $\nabla, \mathbf{u}$ and $\omega$ are all 3-D vectors. Ertel's PV does not require a small Rossby number, but it does require that $\rho$ be conserved. The conservation equation for $\mathrm{PV}$ is

$$
\frac{\partial q}{\partial t}+\mathbf{u} \cdot \nabla q=F
$$

so that PV is conserved following the flow, barring forcing or dissipation, $F$.

In this thesis we focus on the shallow water limit, in which horizontal length scales are much larger than vertical length scales, so that vertical gradients dominate. This limit is relevant to this thesis as we focus on phenomena with large spatial scales $0(100 \mathrm{~km})$ relative to the depth of the ocean $(\approx 5 \mathrm{~km})$. We further assume that the ocean is in hydrostatic balance and that isopycnals are material surfaces, so that PV reduces to

$$
q=\frac{f+\zeta}{\rho} \frac{\partial \rho}{\partial z}=\frac{f+\zeta}{h}
$$

where $\mathrm{h}$ is the vertical distance between isopycnal surfaces, and $\zeta=\frac{\partial v}{\partial x}-\frac{\partial u}{\partial y}$, is the vertical component of the relative vorticity. In Chapter 3, we further approximate PV by $\frac{f}{h}$, and use changes in layer thickness between isopycnals to diagnose changes in PV.

The model used in Chapter 4 is in the quasi-geostrophic limit, in which the flow is assumed to be nearly in geostrophic balance, and perturbations in layer depth are much smaller than the total water depth. The small departures from geostrophy allow the system to evolve in time. Quasi-Geostrophic PV (QGPV) can be approximated from shallow-water $\mathrm{PV}$, as shown in Vallis (2006):

$$
\frac{f+\zeta}{H\left(1+h^{\prime} / H\right)} \approx \frac{1}{H}(f+\zeta)\left(1-h^{\prime} / H\right) \approx \frac{1}{H}\left(f_{0}+\beta y+\zeta-f_{o} h^{\prime} / H\right)
$$

where $H$ is the mean depth of a layer with constant density, $h^{\prime}$ denotes deviations from this mean, $f_{0}$ is the mean Coriolis parameter and $\beta$ is the linearized Coriolis gradient. In this approximation we have used that $h^{\prime} / H \ll 1$, and only kept terms that are $O\left(f_{0} R o\right)$ or 
larger. Since $f_{0} / H$ is a constant, the QGPV is commonly defined as

$$
Q G P V=\beta y+\zeta-f_{o} h^{\prime} / H
$$

QGPV is conserved along geostrophic flow contours absent forcing or dissipation, and is a linear function of the geostrophic streamfunction, so that QGPV can be inverted to determine the flow. The quasi-geostrophic framework facilitates the interpretation of dynamics in terms of PV, and because of their simplicity they were the first equations used to model the effects of eddies on the mean circulation (Holland, 1978). In Chapter 4, we use the two-layer QGPV equations to study the effects of the circulation driven by Gulf Stream eddies on the DWBC.

The essence of western boundary current theory can be explained through the conservation of $\mathrm{PV}$. The conservation of $\mathrm{PV}$ implies that flow cannot cross contours of $f$, or lines of latitude, without a compensating change in another component of $\mathrm{PV}$, or the action of forcing or dissipation. In western boundary current theory, changes in $f$ are allowed by dissipation of PV from friction in the western boundary current. For example, the Gulf Stream's northward motion, and hence increasing $f$ is delivered by the positive PV (vorticity) associated with friction acting to counter the Gulf Stream. Importantly, the PV injected by friction on the western boundary is the correct sign to balance change in planetary PV associated with meridional motion regardless of the direction of the current or hemisphere. Indeed, western boundary currents are observed in each ocean basin in each hemisphere.

\subsection{Scientific context}

\section{The wind-driven circulation}

In Stommel's theory for the wind-driven circulation, the wind forcing over the ocean basin is dissipated in an intense western boundary current by bottom friction. Stommel (1948)'s theory and its extension by Munk (1950), were so successful because of their simplicity and ability to reproduce the main features of the circulation, but they underestimated the Gulf Stream's transport. Both theories also assumed a homogeneous ocean with flat bathymetry, in order to make the problem tractable.

Several theoretical developments addressed these limitations. Fofonoff (1954) showed that inertial basin modes can arise when the nonlinear advective term is included, without bounds on the magnitude of transport. Holland (1972) suggested that adding stratification and bathymetry would correct the Gulf Stream transport. Finally, Holland and Rhines (1980) demonstrated that eddies could force recirculations about an unstable jet, which can also contribute to the transport of the Gulf Stream.

There is an appreciation for all of these developments in the recent literature, and ad- 
vances in modeling the ocean have enabled the detailed diagnosis of PV transformations in ocean models (Czaja and Hausmann, 2009; Deremble et al., 2014; Yeager, 2015; Schoonover et al., 2015). However, the connection between the classical balances of Stommel and Munk and the detailed PV maps in ocean models is not straightforward, with the interpretation of the dynamical balance depending on the mathematical framework (Jackson et al., 2006). It also remains a challenge to quantify large-scale balances from observations (Wunsch, 2011; Gray and Riser, 2014).

In Chapter 2 we consolidate the classical theories of Stommel and Munk with corrections for non-linearity, stratification, and bathymetry. We derive a budget framework for the depth-averaged vorticity, which simplifies to shallow-water PV in the limit of a homogeneous ocean layer, facilitating comparison with classical theories formulated in flat, homogeneous oceans. We diagnose the size of budget terms from observations and a state estimate model that incorporates observations, and find that a source of positive vorticity, such as friction is necessary to balance the negative advective vorticity flux of the Gulf Stream provided by wind stress forcing over the subtropical gyre. At the same, we find that the Gulf Stream has a significant inertial component.

\section{The deep circulation}

In the remaining chapters, the focus turns to the deep circulation, where the ocean is shielded from direct forcing by the atmosphere, and the PV structure is shaped by high-latitude sources, bathymetry and the effects of eddies.

As described in Section 1.2, PV is conserved along isopycnals. The PV of a particular isopycnal can only be altered at its boundaries, where it intersects with the sea surface or bathymetry. This principle was the focus of the classical thermocline theory of Luyten et al. (1983), in which deep motions and properties are set at their high-latitude outcrops, and unventilated regions are quiescent. At the same time, there was growing appreciation for the ability of eddies to transfer momentum to the deep ocean and homogenize PV within closed PV contours. In particular, Holland and Rhines (1980) demonstrated that eddies shed by an unstable Gulf Stream could force recirculation gyres at its flanks. Within these recirculation gyres, which formed closed contours of PV, PV was homogenized by the eddies' weak diffusion (Rhines and Young, 1982).

PV maps charted from observations indicated the relevance of both of these viewpoints. In their maps of PV on isopycnals, McDowell et al. (1982) highlighted the homogenization of PV at depth, but also pointed out that PV is set at isopycnal outcrops. This PV landscape is particularly relevant to the DWBC. The DWBC is composed of several water masses that are formed in the high latitude North Atlantic, referred to collectively as North Atlantic deep water. As these newly formed deep waters travel southward, they are subducted and shielded from atmospheric forcing. 
Talley and McCartney (1982) showed that Labrador Sea Water, one of the water masses in the DWBC, could be traced along the western boundary of the North Atlantic into the tropics using its low PV signature. Their maps also showed the spreading of Labrador Sea Water properties into the interior North Atlantic and they hypothesized that mixing across the Gulf Stream was contributing to this spreading. Recently, Bower et al. (2009) highlighted the spreading of Labrador Sea Water at depth in observations from neutrallybuoyant floats, and the deep recirculation gyres with homogeneous PV are thought to play an important role in setting the pathways for this spreading (Gary et al., 2011). This work questioned the extent to which changes in water mass properties could be detected downstream in the subtropical gyre, because of the apparently diffuse patterns of Labrador Sea Water spreading.

In Chapters 3 and 4, we address questions that arose from this body of literature:

1. To what degree is the spreading of North Atlantic deep water affected by advective (mean) and diffusive (eddy) processes?

2. How do eddy-driven recirculation gyres affect the spreading of North Atlantic deep water?

First, in Chapter 3 we quantify travel times and stirring in the DWBC from a series of water mass observations. We focus on anomalously fresh and cold deep Labrador Sea Water that was formed in the early 1990s in the Labrador Sea and trace it along the path of the DWBC using temperature, salinity and layer thickness (or inverse PV) properties. The primary data sets we use are from the central Labrador Sea, where observations date back to the 1960 's, and at Line W, which is at $39^{\circ} \mathrm{N}$ on the continental slope southeast of New England, where observations are available since the mid 1990's. Because of the length of these datasets, we are able to interpret the data using an advective-diffusive model and show quantitatively that advection along the DWBC and stirring with the interior both play leading order roles in the spreading of Labrador Sea Water.

In Chapter 4, we use a quasi-geostrophic process model to isolate the interaction between the DWBC and the Northern Recirculation Gyre (NRG) of the Gulf Stream. The NRG coexists with the DWBC at depth and corresponds to a region of homogeneous PV directly off-slope from the DWBC (Hogg, 1983; Lozier, 1997; Gary et al., 2011). Using our model, we investigate how the eddy-driven NRG adjusts to the presence of a slope and how this adjustment could affect a DWBC on the slope. We find that the NRG can homogenize PV into the slope and that there is an off-slope eddy PV flux associated with this homogenization. This off-slope eddy PV flux could contribute to spreading of DWBC water into the interior observed in Chapter 3 and in the literature (Talley and McCartney, 1982; Hogg et al., 1986; Bower et al., 2009). The dynamical framework that we present in Chapter 4 could be used to inform future observations and realistic models. 


\subsection{Thesis Overview}

This thesis is comprised of three seemingly different studies of North Atlantic western boundary currents, the Gulf Stream and the Deep Western Boundary Current. However, each chapter deals with the basic question:

\section{"What shapes the large scale distribution of}

Potential Vorticity in the North Atlantic?"

Each chapter connects with this question in a different way:

- Chapter 2 is a description of the large scale balance of vorticity in the wind-driven subtropical gyre, which simplifies to a balance of PV for a homogeneous layer.

- In Chapter 3, we quantify advective and eddy stirring time scales for the spreading of North Atlantic deep water from observations, using PV as well as water mass composition as tracers. This analysis informs our understanding of the PV distribution along the path of the DWBC.

- Chapter 4 is a description of the PV dynamics of simplified subtropical North Atlantic, which includes only a Gulf Stream, its recirculation gyres and a DWBC on a slope.

Finally, Chapter 5 brings the results of each chapter together, with a discussion of their implications as well as potential future directions for this research. 


\section{Chapter 2}

\section{A Vorticity Budget for the Western North Atlantic Based on Observations}




\section{$2.1 \quad$ Abstract}

An integrated, time-averaged vorticity budget for the vertically averaged velocity is developed to diagnose dynamical features of the North Atlantic subtropical gyre. The mathematical framework simplifies to a budget for depth-integrated shallow-water Potential Vorticity (PV) in the limit that the study volume has homogeneous density. This renders our framework analogous to classical theories of gyre circulation in flat, barotropic oceans (e.g Stommel, 1948; Munk, 1950; Fofonoff, 1954). The focus is on two of the terms that persist in this limit: the lateral advective vorticity flux out of the volume and the wind stress forcing.

The study volume is west of the WOCE/CLIVAR section A22, nominally along $66^{\circ} \mathrm{W}$ and above the $\sigma_{0}=27 \mathrm{~kg} \mathrm{~m}^{-3}$ potential density anomaly surface; the Gulf Stream extension flows out of this volume in the north of the section. Advective vorticity fluxes out of the volume are quantified using hydrographic and ADCP velocity data from the A22 section. The contour integral of the wind stress term in the vorticity budget is calculated from a variety of data products, both for the western region and over the whole subtropical gyre.

Because the calculated advective vorticity flux is robustly negative, and the wind stress forcing over the western region is comparatively small, a positive source of vorticity in the western region is inferred. The advective vorticity flux out of the western region is on the same order as the wind stress forcing over the eastern portion of the gyre, consistent with a large-scale balance between a negative source of vorticity from wind stress forcing and a positive source of vorticity in the western region. Additionally, the form of the vorticity flux indicates that the Gulf Stream has a significant inertial component.

An analogous budget is calculated from the ECCO state estimate, and the terms are found to vary significantly in time. The ECCO model is also used to diagnose the size of the significant remaining budget terms, and we find a primary balance between advective vorticity flux and lateral friction in the western region in the ECCO model. Taken together, the observations and ECCO model indicate that while the Gulf Stream is very inertial, a source of positive vorticity in the west, such as friction, is necessary to close its vorticity budget. 


\subsection{Introduction}

In 1948, Henry Stommel proposed an elegant explanation for the westward intensification of ocean currents in gyres. This explanation can be conveniently framed in terms of Potential Vorticity (PV), a dynamical tracer that is conserved barring mechanical forcing or dissipation. In the interior of subtropical gyres, Sverdrup (1947) had predicted that the anticyclonic wind pattern caused convergence in the Ekman layer, causing squashing of the water column, and southward flow throughout the ocean depth. Stommel (1948) showed that this southward flow could be returned in an intense northward jet on the western side of the basin, where the change of PV in this intense northward jet is balanced by bottom friction. More generally, in a flat ocean, only on the western side of the basin does friction inject the correct sign of PV to allow meridional motion in compensation for the Sverdrup interior circulation.

A few years layer, Munk (1950) extended this theory, replacing linear bottom friction with lateral eddy viscosity. The viscosity required to obtain a realistic solution corresponds to the viscosity of eddies that are the same size as the Gulf Stream width. Indeed, eddies of this size are created by Gulf Stream meanders. Ironically, this linear theory pointed to the importance of non-linearity in the system.

These early theories were linear so that analytic solutions were tractable, but the importance of nonlinear terms and inertia were also recognized. To demonstrate that purely inertial gyre solutions could be found, Fofonoff (1954) assumed a linear relationship between streamfunction and potential vorticity and derived inertial basin modes. Holland (1972) demonstrated that topography and stratification could act in tandem to allow inertial recirculation gyres. Marshall and Nurser (1986) later showed that steady inertial solutions also existed in a stratified quasi-geostrophic ocean. However, inertial theories do not predict the east-west asymmetry of ocean gyres, nor do they discount the possibility that friction plays a dynamically important role. In order to quantify the inertial component of the Gulf Stream, Charney (1955) diagnosed an inertial boundary current width for the Florida Current. He also argued that once it leaves the coast, the Gulf Stream is no longer steady and cannot be considered a boundary layer.

The relative sizes of the frictional and inertial boundary layers can be compared to diagnose the relative roles of friction and inertia in a gyre circulation. As discussed in Fox-Kemper and Pedlosky (2004), in ocean models with constant viscosity, if the inertial boundary layer is larger than the frictional boundary layer, i.e. the advection of PV is stronger than the dissipation of PV, the inertial boundary layer expands until it fills the entire domain. Fox-Kemper and Pedlosky (2004) overcame this "inertial runaway" solution by increasing the viscosity at the western boundary, to represent unresolved boundary dissipation and interaction with topography. In their model, realistic barotropic gyre strengths 
can be achieved even when the viscous boundary layer is smaller than the inertial boundary layer (i.e. a viscous sublayer), as eddies flux PV into the high friction boundary layer, where it can be dissipated more effectively. Fox-Kemper and Pedlosky (2004) illustrate that inertia and friction can co-exist effectively in a balanced gyre circulation, and that friction is necessary even in a strongly inertial system.

Once stratification and topography are included in circulation models, the expression for PV becomes more complex. The PV for a flat-bottomed barotropic ocean is absolute vorticity, $f+\zeta$, where $f$ is the planetary vorticity and $\zeta$ the relative vorticity of the flow. The PV in an ocean with discrete layers of homogeneous density and/or bathymetry includes the effect of vortex stretching, as was first introduced in Rossby (1936). This form of PV is also known as shallow-water PV, and is written, $q=\frac{f+\zeta}{h}$, where $h$ is the layer thickness or water depth. In an ocean with continuous stratification and topography, the relevant form of PV is due to Ertel (1942), $q=(\mathbf{f}+\nabla \times \mathbf{u}) / \rho \cdot \nabla \rho$, where $\mathbf{f}$ is the full planetary vorticity vector, $\mathbf{u}$ is the velocity vector and $\rho$ is density. In this case, the stretching term is rendered continuous and contributions from horizontal vorticity may become significant. In a stratified ocean, the upper water column is shielded from the ocean floor, complicating the extraction of PV through interaction with topography and friction. In a flat-bottomed barotropic model ocean at rest, PV contours correspond to $f$ contours. When bathymetry is added, the contours of PV become $f / H$ contours, where $\mathrm{H}$ is the full ocean depth.

Several studies have diagnosed the fluxes of Ertel PV in the North Atlantic. Marshall et al. (2001a) calculate PV fluxes on isopycnal surfaces in a model of the North Atlantic and Polton and Marshall (2003) calculate vertical PV fluxes on depth levels in an idealized gyre model. Czaja and Hausmann (2009) calculate PV fluxes through the surface of the North Atlantic using climatologies and Deremble et al. (2014) calculate PV fluxes in an eddy-resolving model. Despite their differences in approach, these studies agree on the basic balance of PV entry through the interior North Atlantic and PV exit in the western boundary. They also emphasize the significance of buoyancy forcing and recirculation, especially Deremble et al. (2014). Our estimates of vorticity flux from the western North Atlantic into the interior compare favorably with Deremble et al.'s, as we discuss in Section 2.7.

Our study is based around an equation for the vorticity of the depth-averaged flow, as derived in Section 2.3, not full Ertel PV. If our study volume were a layer of homogeneous density, our budget of vorticity for the depth-averaged flow would simplify to a budget of depth-integrated shallow-water PV. In this limit, only wind stress forcing, vorticity advection and friction remain. Given the success of models with a low number of discrete density layers in reproducing the main features in the North Atlantic, such as the thermocline theory of Luyten et al. (1983), we use this limit to guide the interpretation of our results.

The vorticity equation for the depth-averaged flow is notably different than the barotropic vorticity equation which forms the basis of studies that argue that western boundary currents 
are inviscid (e.g. Hughes and de Cuevas, 2001; Lu and Stammer, 2004; Jackson et al., 2006; Yeager, 2015; Schoonover et al., 2015). In the barotropic vorticity framework, the basin scale wind stress torque is balanced by bottom pressure torques, gradients of bottom pressure along isobaths. Jackson et al. (2006) and Yeager (2015) explain the relationship between barotropic vorticity budgets and PV budgets; barotropic vorticity budgets highlight the relative contributions of the vorticity and thickness components of PV to PV conservation. In Section 2.3 we will detail the mathematical relationship and further discuss the connection between the vorticity equation for depth-averaged flow, which is our focus and analogous to $\mathrm{PV}$, and the vorticity of the depth-integrated flow, which is the focus of barotropic vorticity studies.

Other large scale balances have focused on testing the validity of the Sverdrup balance, a vorticity balance in which wind input of vorticity causes depth integrated meridional transport (e.g. Sverdrup, 1947; Leetmaa et al., 1977; Wunsch and Roemmich, 1985; Schmitz et al., 1992; Hautala et al., 1994; Wunsch, 2011; Thomas et al., 2014). Most recently, Gray and Riser (2014) tested Sverdrup balance using Argo data, concluding (as did Wunsch (2011) and Thomas et al. (2014)) that although it is not axiomatic, Sverdrup balance is relevant in the interior subtropical and tropical ocean. Wunsch and Heimbach (2013b) emphasize that Sverdrup balance must fail in a basin-wide sense in order for the flow field to be consistent with the measured meridional heat transport.

In this work, we turn from the vorticity balance in the interior to focus on the western boundary. Our analysis is based around the A22 WOCE/CLIVAR (World Ocean Circulation Experiment/Climate Variability \& Predictability) hydrographic section. The A22 cruise track lies nominally along $66^{\circ} \mathrm{W}$ (see Figure 2-1) and cuts off a portion of the North Atlantic to its west. Because A22 is the only lateral oceanic boundary of this western region, we can calculate the advective vorticity flux out of the region from A22 cruise data. The other boundaries of the study volume are the ocean surface, the American coastline, and an isopycnal that lies within the main pycnocline. We calculate the mean wind stress input of vorticity at the surface from a variety of wind stress products over the region to the west of A22, as well as over the full subtropical North Atlantic to provide a point of reference. The remaining terms cannot reliably be assessed from observations and their significance and potential contribution to the vorticity budget are discussed in Section 2.6.

To complement this calculation, we develop an analogous vorticity budget using data from the ECCO v4 (Estimating the Circulation and Climate of the Ocean) state estimate. ECCO $v 4$ is a roughly $1^{\circ}$ resolution global adjoint state estimate on a cubed-sphere grid with 50 vertical levels that is based on the Massachusetts Institute of Technology general circulation model (MITgcm) and incorporates most available ocean data (Wunsch and Heimbach, 2013a). Despite its relatively coarse resolution, the ECCO model has been shown to reproduce the essential features of the North Atlantic circulation, in particular the trans- 


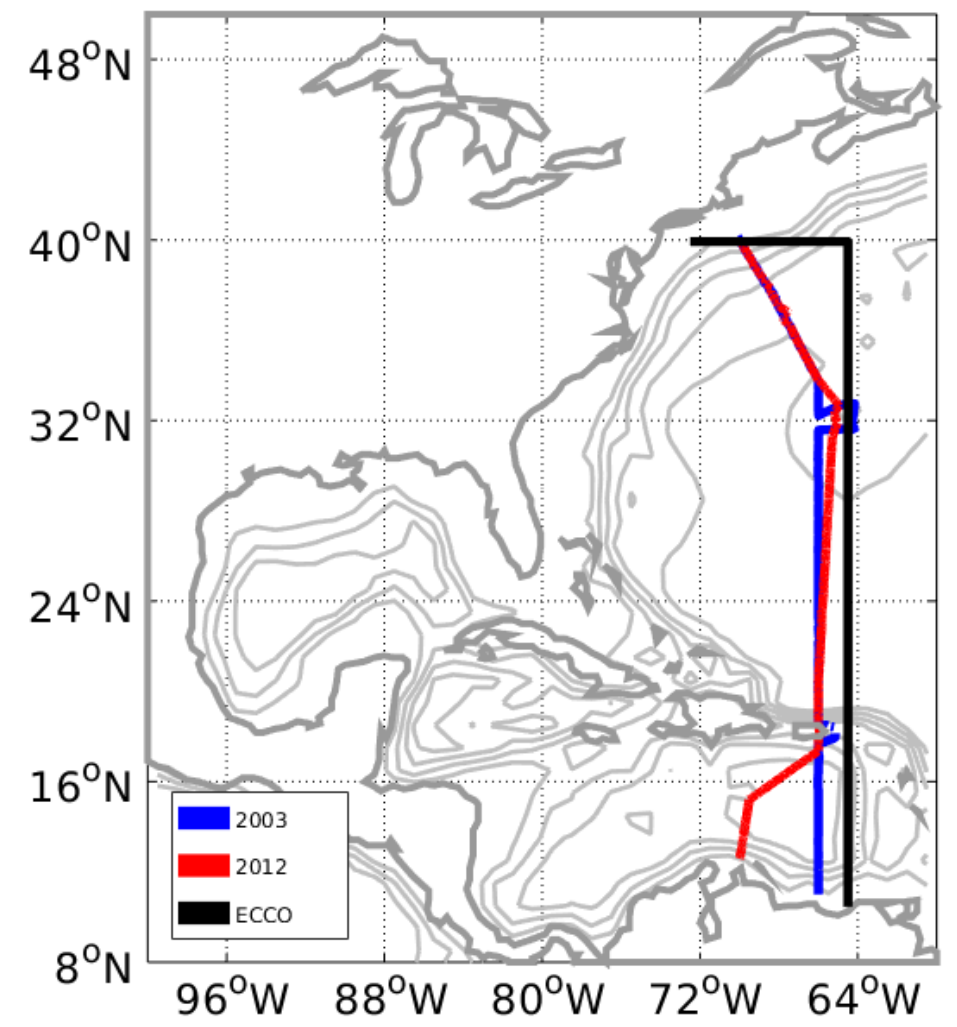

Figure 2-1: Map of WOCE/CLIVAR A22 cruise tracks and the ECCO boundary. The 2003 cruise track is in blue, 2012 in red, and the ECCO boundary in black. Light grey lines are bathymetric contours starting at $1000 \mathrm{~m}$ depth in intervals of $1000 \mathrm{~m}$.

ports of the Gulf Stream and full overturning circulation (Wunsch and Heimbach, 2013b). The run period is 19 years (1992-2010), and we analyze monthly averages. We focus on the volume west of $64.5^{\circ} \mathrm{W}$ in ECCO so as to avoid the control volume boundary intersecting islands in the Caribbean. We compare the relative sizes of the terms in the ECCO state estimate to the relative sizes of the terms in the observations and use ECCO to gauge the level of temporal variability in the budget terms. We also use the ECCO data to diagnose the size of remaining budget terms, as detailed in Section 2.6.

We choose an isopycnal as the lower boundary of our study volume because diapycnal velocities tend to be smaller than vertical velocities in the subtropical ocean. This choice minimizes fluxes through the lower boundary. So, although buoyancy forcing does not enter our vorticity budget explicitly, it does enter in the sense that it can change the position of the isopycnal which bounds our study volume from below. The isopycnals we choose as the lower boundary are the shallowest isopycnal that do not outcrop in the study region over the course of the year. If the isopycnal were to outcrop, that would introduce a lateral 
boundary along which we do not have observations. By choosing an isopycnal that does not outcrop in the region of study, we ensure that the vorticity flux across the A22 hydrographic section, and corresponding section in the ECCO model are the sole lateral fluxes out of our study volume. We also want to take full advantage of the A22 data and ensure that the relevant features of the wind-driven gyre (e.g. the Gulf Stream) are included above the isopycnal boundary that we select. With these considerations in mind, we select the $\sigma_{0}=27 \mathrm{~kg} \mathrm{~m}^{-3}$ potential density anomaly surface relative to 0 dbar, an isopycnal which does not outcrop in the region, according to the mixed layer climatology presented in Holte

and Talley (2009). In the ECCO model, we use the $\sigma_{0}=27.15 \mathrm{~kg} \mathrm{~m}^{-3}$ potential density anomaly surface as the lower boundary of the study volume as the ECCO density field is biased light compared to the observations in this range. The depth of the isopycnal used in the ECCO model compares well with the Gouretski and Koltermann (2004) climatological value, which we use to calculate wind stress forcing from observational data products, as can be seen in Figure 2-2. Our choices of bounding isopycnal are similar to that used in Gray and Riser (2014) and Rhines and Young (1982), who approximated the top layer in Luyten et al.'s 1983 ventilated thermocline theory.

The rest of this chapter details the calculation of budget terms and their interpretation. We begin by presenting the mathematical framework in Section 2.3. In Section 2.4, we discuss the contribution of wind stress to the vorticity budget, in Section 2.5 the lateral flux of vorticity out of the western region and in Section 2.6 the remaining budget terms. We discuss the implications of our results in Section 2.7 .

\subsection{Mathematical framework}

\section{Derivation of depth-averaged vorticity framework}

To diagnose important elements of the large scale balance of vorticity in the western subtropical North Atlantic, we calculate the size of terms in a horizontally-integrated equation for the vorticity of the vertically-averaged flow. The volume we analyze spans the distance between the air-sea interface and the depth of the $\sigma_{0}=27 \mathrm{~kg} \mathrm{~m}^{-3}$ potential density anomaly surface, at $z=-h(x, y, t)$. We make the rigid-lid approximation, so that the air-sea interface is at $z=0$. We begin with the horizontal, Boussinesq momentum equations,

$$
\mathbf{u}_{t}+u \mathbf{u}_{x}+v \mathbf{u}_{y}+w \mathbf{u}_{z}+f \mathbf{k} \times \mathbf{u}=-\frac{\nabla p}{\rho_{0}}+\frac{\boldsymbol{\tau}_{z}}{\rho_{0}}+A_{h} \nabla^{2} \mathbf{u}
$$

where $\mathbf{u}=(u, v, 0)$ is the horizontal velocity vector and $\nabla=\left(\partial_{x}, \partial_{y}, 0\right)$ is the horizontal gradient operator. The vertical speed is $w, \mathbf{k}$ is the unit vector in the vertical direction, $\rho_{0}$ is a constant reference velocity, $p$ is pressure, $\boldsymbol{\tau}$ is stress and $A_{h}$ is the laplacian viscosity coefficient. Derivatives are denoted by subscripts. To obtain an equation for the vorticity of 

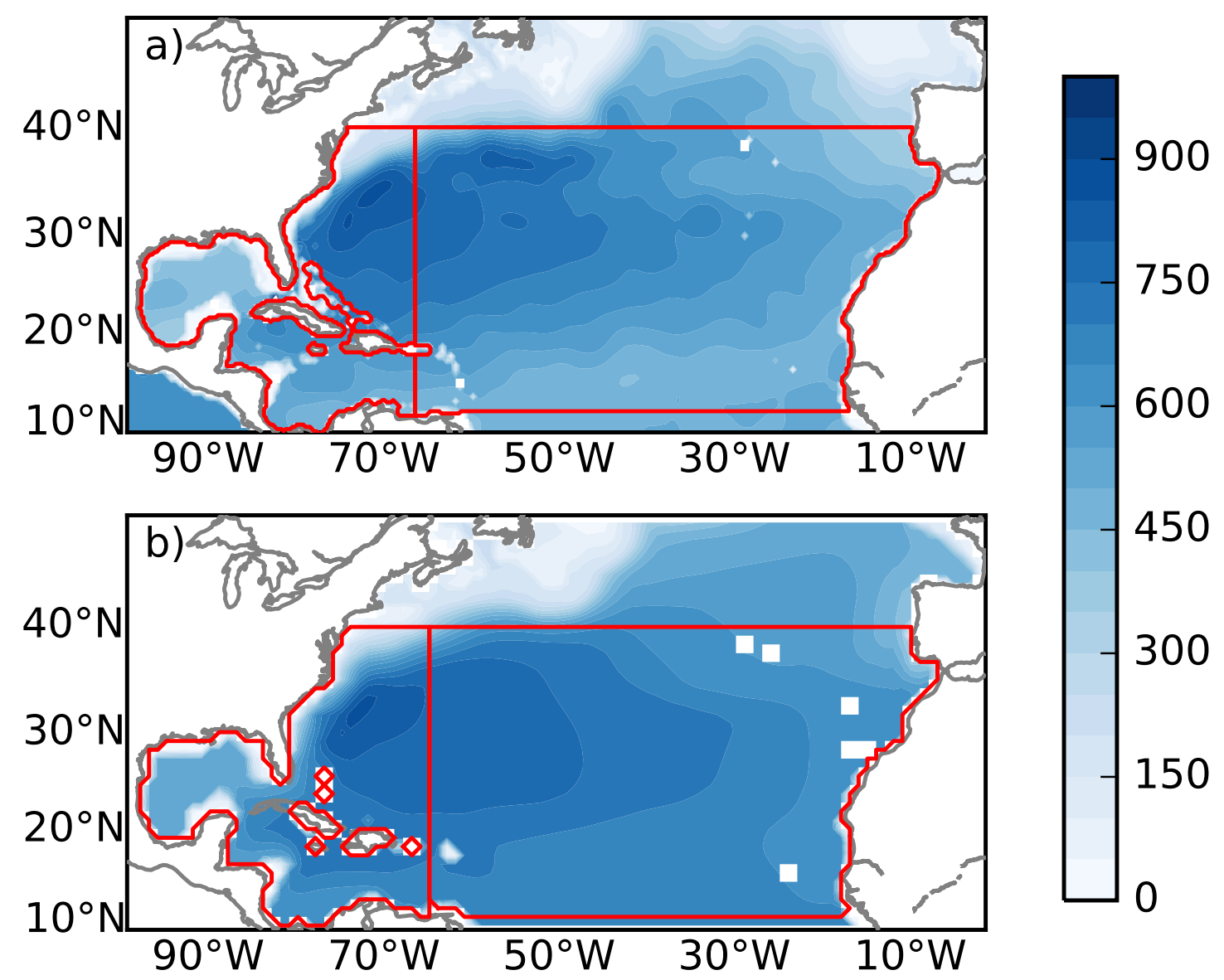

Figure 2-2: Depth of the study volume in the Gouretski and Koltermann (2004) climatology and ECCO state estimate. Contour lines are every $50 \mathrm{~m}$. The red lines show the contours used for calculation of the wind stress forcing budget term. The minimum layer thickness used is $5 \mathrm{~m}$, the shallowest gridpoint in the ECCO state estimate. a) Mean layer thickness from the surface to the $\sigma_{0}=27 \mathrm{~kg} \mathrm{~m}^{-3}$ potential density anomaly surface in the Gouretski and Koltermann (2004) climatology, used to calculate wind stress forcing from SCOW, ERA and MERRA wind stress products. b) Mean layer thickness from the surface to the $\sigma_{0}=27.15 \mathrm{~kg} \mathrm{~m}^{-3}$ potential density anomaly surface in the ECCO state estimate.

the depth-averaged flow in our study volume, we will integrate equation 2.1 vertically from $z=-h(x, y, t)$ to $z=0$, divide by $h(x, y, t)$ and then integrate around the horizontal area of the volume to derive the area integrated vorticity equation for the depth-averaged velocity.

Several auxiliary equations will be useful when integrating the momentum equation vertically from the isopycnal at $z=-h(x, y, t)$. Namely, the equation of motion for the isopycnal at $z=-h(x, y, t)$ :

$$
h_{t}+\nabla \cdot(h \overline{\mathbf{u}})=\omega
$$

where $\omega(x, y, t)$ is a diapycnal velocity through the isopycnal at $z=-h(x, y, t)$ and the overline denotes the vertical average, e.g. $\overline{\mathbf{u}}=\frac{1}{h} \int_{-h}^{0} \mathbf{u} d z$. Further, for any variable $\boldsymbol{\theta}(x, y, z, t)$, 
the vertical integral of the time derivative and advective terms may be written as

$$
\int_{-h}^{0}\left[\boldsymbol{\theta}_{t}+u \boldsymbol{\theta}_{x}+v \boldsymbol{\theta}_{y}+w \boldsymbol{\theta}_{z}\right] d z=(h \overline{\boldsymbol{\theta}})_{t}+(h \overline{u \boldsymbol{\theta}})_{x}+(h \overline{v \boldsymbol{\theta}})_{y}-\omega \boldsymbol{\theta}^{h}
$$

where superscripts denote the $\mathrm{z}$ level at which a variable is evaluated, i.e. $\boldsymbol{\theta}^{h}$ is $\boldsymbol{\theta}$ evaluated at $z=-h(x, y, t)$. Equation 2.3 can be proved using 2.2 and the Leibniz integral rule, which is, for reference,

$$
\int_{a(x)}^{b(x)} f(x, z)_{x} d z=\left(\int_{a(x)}^{b(x)} f(x, z) d z\right)_{x}+f(b(x), x) b_{x}-f(a(x), x) a_{x} .
$$

Integrating the first four terms in equation 2.1 vertically, using equations 2.2 and 2.3 to simplify, yields

$$
\begin{gathered}
\int_{-h}^{0}\left[\mathbf{u}_{t}+u \mathbf{u}_{x}+v \mathbf{u}_{y}+w \mathbf{u}_{z}\right] d z=(h \overline{\mathbf{u}})_{t}+(h \overline{u \mathbf{u}})_{x}+(h \overline{v \mathbf{u}})_{y}-\omega \mathbf{u}^{h} \\
=h_{t} \overline{\mathbf{u}}+h \overline{\mathbf{u}}_{t}+h(\overline{\mathbf{u}} \cdot \nabla) \overline{\mathbf{u}}+\overline{\mathbf{u}} \nabla \cdot(h \overline{\mathbf{u}})+\nabla \cdot\left(h \overline{\mathbf{u}^{\prime} \mathbf{u}^{\prime}}\right)-\omega \mathbf{u}^{h} \\
=h\left(\overline{\mathbf{u}}_{t}+(\overline{\mathbf{u}} \cdot \nabla) \overline{\mathbf{u}}\right)+\nabla \cdot\left(h \overline{\mathbf{u}^{\prime} \mathbf{u}^{\prime}}\right)+\overline{\mathbf{u}}\left(h_{t}+\nabla \cdot(h \overline{\mathbf{u}})\right)-\omega \mathbf{u}^{h} \\
=h\left(\overline{\mathbf{u}}_{t}+(\overline{\mathbf{u}} \cdot \nabla) \overline{\mathbf{u}}\right)+\nabla \cdot\left(h \overline{\mathbf{u}^{\prime} \mathbf{u}^{\prime}}\right)+\omega\left(\overline{\mathbf{u}}-\mathbf{u}^{h}\right),
\end{gathered}
$$

where

$$
\nabla \cdot\left(h \overline{\mathbf{u}^{\prime} \mathbf{u}^{\prime}}\right)=\left(\left(h \overline{u^{\prime} u^{\prime}}\right)_{x}+\left(h \overline{u^{\prime} v^{\prime}}\right)_{y}\right) \mathbf{i}+\left(\left(h \overline{u^{\prime} v^{\prime}}\right)_{x}+\left(h \overline{v^{\prime} v^{\prime}}\right)_{y}\right) \mathbf{j}
$$

Hence the vertical integral of equation 2.1 is

$$
\begin{aligned}
& h\left(\overline{\mathbf{u}}_{t}+(\overline{\mathbf{u}} \cdot \nabla) \overline{\mathbf{u}}\right)+\nabla \cdot\left(h \overline{\mathbf{u}^{\prime} \mathbf{u}^{\prime}}\right)+\omega\left(\overline{\mathbf{u}}-\mathbf{u}^{h}\right)+f \mathbf{k} \times h \overline{\mathbf{u}} \\
& =-\frac{1}{\rho_{0}}\left(\nabla(\bar{p} h)-p^{h} \nabla h+\boldsymbol{\tau}^{0}-\boldsymbol{\tau}^{h}\right)+\int_{-h}^{0}\left(A_{h} \nabla^{2} \mathbf{u}\right) d z,
\end{aligned}
$$

where we have applied the Leibniz rule to the pressure gradient term, which results in the form stress term, $p^{h} \nabla h . \bar{p}$ is the vertical average of pressure, $\bar{p}=\frac{1}{h} \int_{-h}^{0} p d z$.

Dividing by $h(x, y, t)$ to get the vertical average of equation 2.1 yields

$$
\begin{gathered}
\overline{\mathbf{u}}_{t}+(\overline{\mathbf{u}} \cdot \nabla) \overline{\mathbf{u}}+f \mathbf{k} \times \overline{\mathbf{u}}+\frac{1}{h}\left(\nabla \cdot\left(h \overline{\mathbf{u}^{\prime} \mathbf{u}^{\prime}}\right)+\omega\left(\overline{\mathbf{u}}-\mathbf{u}^{h}\right)\right) \\
=-\frac{1}{\rho_{0} h}\left(\nabla(\bar{p} h)-p^{h} \nabla h+\boldsymbol{\tau}^{0}-\boldsymbol{\tau}^{h}\right)+\overline{A_{h} \nabla^{2} \mathbf{u}}
\end{gathered}
$$

Integrating equation 2.6 around a contour gives a circulation equation for the vertically av- 
eraged velocity. We denote the vertical component of the vorticity of the vertically averaged relative velocity with a tilde, i.e. $\widetilde{\zeta}=\bar{v}_{x}-\bar{u}_{y}$, as well as its circulation, $\widetilde{\Gamma}=\iint \widetilde{\zeta} \mathbf{k} \cdot \mathbf{d} \mathbf{A}=$ $\oint \overline{\mathbf{u}} \cdot \mathbf{d s}$, where $\mathbf{d s}$ is the unit vector parallel to the contour. Throughout the manuscript we will refer to the vorticity of the depth-averaged flow, $\widetilde{\zeta}$, as vorticity for simplicity, but it is worth noting that the vorticity of the depth-averaged flow is not equivalent to its depth-averaged vorticity as

$$
\bar{\zeta}=\frac{1}{h} \int_{-h}^{0}\left(v_{x}-u_{y}\right) d z=\bar{v}_{x}-\bar{u}_{y}-v^{h} h_{x}+u^{h} h_{y}
$$

by the Leibniz integral rule.

The contour integral of the advective and Coriolis terms can be simplified by taking advantage of the identity

$$
(\overline{\mathbf{u}} \cdot \nabla) \overline{\mathbf{u}}=\frac{1}{2} \nabla|\overline{\mathbf{u}}|^{2}+\widetilde{\zeta} \mathbf{k} \times \overline{\mathbf{u}}
$$

so that

$$
\oint((\overline{\mathbf{u}} \cdot \nabla) \overline{\mathbf{u}}+f \mathbf{k} \times \overline{\mathbf{u}}) \cdot \mathbf{d} \mathbf{s}=\oint\left(\frac{1}{2} \nabla|\overline{\mathbf{u}}|^{2}+\widetilde{\zeta} \mathbf{k} \times \overline{\mathbf{u}}+f \mathbf{k} \times \overline{\mathbf{u}}\right) \cdot \mathbf{d} \mathbf{s}
$$

Grouping like terms, using Stokes' theorem and the fact that the curl of a gradient is zero, equation 2.7 can be simplified to

$$
=\oint\left(\frac{1}{2} \nabla|\overline{\mathbf{u}}|^{2}+(f+\widetilde{\zeta}) \mathbf{k} \times \overline{\mathbf{u}}\right) \cdot \mathbf{d} \mathbf{s}=\iint(\nabla \times(f+\widetilde{\zeta}) \mathbf{k} \times \overline{\mathbf{u}}) \cdot \mathbf{k} d A
$$

Applying the vector calculus identity $(\nabla \times Z) \cdot \mathbf{k}=\nabla \cdot(\mathbf{k} \times Z)$, as $\nabla \times \mathbf{k}=0$, and Gauss' theorem, we simplify equation 2.7 further to

$$
\begin{aligned}
\iint \nabla \cdot((f+\zeta) \mathbf{k} & \times \mathbf{u} \times \mathbf{k}) d A=\iint \nabla \cdot(f+\widetilde{\zeta}) \overline{\mathbf{u}} d A \\
= & \int(f+\widetilde{\zeta}) \overline{\mathbf{u}} \cdot \mathbf{d} \mathbf{n}
\end{aligned}
$$

where $\mathbf{d} \mathbf{n}$ is the unit vector normal to the integration contour, and we have also used the vector identities, $A \times(B \times C)=(A \cdot C) B-(A \cdot B) C$ and $\mathbf{k} \cdot \mathbf{k}=1$. The resulting term is the flux of the absolute vorticity of the depth-averaged velocity by the depth-averaged velocity.

The contour integral of the pressure gradient terms can also be simplified and related to the Joint Effect of Baroclinicity and Relief or "JEBAR" term (Sarkisyan and Ivanov, 1971). Expanding the pressure gradient terms in equation 2.5,

$$
-\frac{1}{\rho_{0} h}\left(\nabla(\bar{p} h)-p^{h} \nabla h\right)=-\frac{1}{\rho_{0} h}\left(h \nabla \bar{p}-\left(\bar{p}-p^{h}\right) \nabla h\right)=-\frac{1}{\rho_{0}} \nabla \bar{p}+h\left(\bar{p}-p^{h}\right) \nabla \frac{1}{h},
$$


where we have used that $\nabla(1 / h)=-\nabla h / h^{2}$. Taking the contour integral of equation 2.8 ,

$\frac{1}{\rho_{0}} \oint\left(-\nabla \bar{p}+h\left(\bar{p}-p^{h}\right) \nabla \frac{1}{h}\right) \cdot \mathbf{d} \mathbf{s}=\frac{1}{\rho_{0}} \iint \nabla\left(h\left(\bar{p}-p^{h}\right)\right) \times \nabla\left(\frac{1}{h}\right) \cdot \mathbf{d} \mathbf{A}=\iint J\left(\chi, \frac{1}{h}\right) d A$,

where $\chi=h\left(\bar{p}-p^{h}\right) / \rho_{0}$, as in Mertz and Wright (1992), for example, and we have used the fact that the curl of a gradient is zero as well as $\nabla \times(A \nabla B)=\nabla A \times \nabla B$. $J$ is the Jacobian operator, $J(A, B)=A_{x} B_{y}-A_{y} B_{x}$. As discussed in Mertz and Wright (1992), the JEBAR term can be interpreted as the "geostrophic component of the correction to the topographic stretching term to account for the fact that the bottom velocity, not the depthaveraged velocity, yields topographic vortex-tube stretching". We will clarify its connection to bottom pressure torques in the next subsection. Note that in our framework, $h$ only corresponds to the ocean bathymetry where the isopycnal at $z=-h$ has grounded, on the western boundary, for example. This term is only identical to JEBAR where this is the case.

In sum, the total integral of equation 2.6 around a fixed surface contour is

$$
\begin{aligned}
& \widetilde{\Gamma}_{t}=-\int(f+\widetilde{\zeta}) \overline{\mathbf{u}} \cdot \mathbf{d} \mathbf{n}-\oint \frac{1}{h}\left(\nabla \cdot\left(h \overline{\mathbf{u}^{\prime} \mathbf{u}^{\prime}}\right)+\omega\left(\overline{\mathbf{u}}-\mathbf{u}^{h}\right)\right) \cdot \mathbf{d} \mathbf{s} \\
& \quad+\iint J\left(\chi, \frac{1}{h}\right) d A+\oint \frac{1}{\rho_{0} h}\left(\boldsymbol{\tau}^{0}-\boldsymbol{\tau}^{h}\right) \cdot \mathbf{d} \mathbf{s}+\oint \overline{A_{h} \nabla^{2} \mathbf{u}} \cdot \mathbf{d s} .
\end{aligned}
$$

This circulation equation for the depth-averaged velocity forms the basis of this work. From left to right, the terms are the time derivative of the circulation of the depth-averaged velocity, the depth-averaged flux of the vorticity of the depth-averaged velocity, a correction due to correlated velocity deviations in depth, an entrainment stress due to diapycnal velocities, the JEBAR term, wind and bottom stress, and lateral viscosity.

If the study volume were a layer of homogeneous density and hence had no vertical shear, this equation would simplify to

$$
\Gamma_{t}=-\int(f+\zeta) \mathbf{u} \cdot \mathbf{d} \mathbf{n}+\oint \frac{1}{\rho_{0} h}\left(\boldsymbol{\tau}^{0}-\boldsymbol{\tau}^{h}\right) \cdot \mathbf{d} \mathbf{s}+\oint \overline{A_{h} \nabla^{2} \mathbf{u}} \cdot \mathbf{d} \mathbf{s}
$$

where terms have canceled and tildes and overlines have been removed because $\mathbf{u}^{\prime}=0$, and $\overline{\mathbf{u}}=\mathbf{u}=\mathbf{u}^{h}$ in this limit. The JEBAR term is zero for a layer of homogeneous density because

$$
\chi=-\frac{1}{2} \widehat{\rho} g h^{2} / \rho_{0}
$$

for a homogeneous layer, where $\widehat{\rho}$ is the constant density of the layer. The only element of $\chi$ that is a function of $x$ and $y$ in this limit is $h$, so that

$$
J E B A R=-\frac{1}{\rho_{0}} \oint \chi \nabla \frac{1}{h} \cdot \mathbf{d} \mathbf{s}=-\oint \frac{1}{2} \frac{\widehat{\rho} g h^{2}}{\rho_{0}}\left(-\frac{\nabla h}{h^{2}}\right) \cdot \mathbf{d} \mathbf{s} \propto \oint \nabla h \cdot \mathbf{d} \mathbf{s}=0 .
$$


Manipulating the first two terms in equation 2.10 sheds some light on its meaning:

$$
\Gamma_{t}+\int(f+\zeta) \mathbf{u} \cdot \mathbf{d} \mathbf{n}=\iint\left((f+\zeta)_{t}+\nabla \cdot(f+\zeta) \mathbf{u}\right) d A=\iint\left((q h)_{t}+\nabla \cdot(q h) \mathbf{u}\right) d A
$$

where $q=\frac{f+\zeta}{h}$. This shows that in the homogeneous layer limit, equation 2.9 reduces to a flux equation for the shallow-water PV of the layer. This limit also reveals the connection of this work to the impermeability theorem (Haynes and McIntyre, 1987), which states that PV cannot be fluxed across isopycnals. This particular form of the impermeability theorem, for shallow-water PV, and its connection of the impermeability theorem for full Ertel PV is clarified in the appendix of Marshall et al. (2001a). Note, however, that as explained in Rhines and Holland (1979), lateral eddy PV thickness fluxes correspond to vertical eddy momentum fluxes. As they warn, "one should not form the impression that eddies do not flux anything of importance across isopycnals".

In this work, we will focus on terms that we can calculate from observations, namely the advective vorticity flux,

$$
-\int(f+\widetilde{\zeta}) \overline{\mathbf{u}} \cdot \mathbf{d} \mathbf{n}
$$

which we calculate from shipboard observations along the eastern boundary of our study volume, and the wind stress forcing,

$$
\oint \frac{\tau^{0}}{\rho_{0} h} \cdot \mathbf{d} \mathbf{s}
$$

which we calculate from a variety of satellite products. We also diagnose these terms in the ECCO state estimate to assess the magnitude of their temporal variability, and calculate the size of the remaining terms in equation 2.9 to form a closed budget for this layer in the ECCO model.

\section{Mathematical connection to depth-integrated vorticity framework}

The meaning of our mathematical framework can be clarified through its connection to the barotropic vorticity equation which forms the basis of many recent studies that argue that western boundary currents are inviscid (e.g. Hughes and de Cuevas, 2001; Lu and Stammer, 2004; Jackson et al., 2006; Schoonover et al., 2015; Yeager, 2015). We can derive an equation that is analogous to the barotropic vorticity equation by taking the contour integral of equation 2.5 before dividing it by $\mathrm{h}$. While the barotropic vorticity equation in Hughes and de Cuevas (2001) is based on an integral over the full water column, here we integrate from the isopycnal at $z=-h(x, y, t)$ to the surface so that the result is comparable to our equation 2.9. 
Taking the contour integral of the advective and Coriolis terms in equation 2.5 yields

$$
\begin{aligned}
& \oint(h(\overline{\mathbf{u}} \cdot \nabla) \overline{\mathbf{u}}+f \mathbf{k} \times h \overline{\mathbf{u}}) \cdot \mathbf{d} \mathbf{s}=\oint\left(h\left(\frac{1}{2} \nabla|\overline{\mathbf{u}}|^{2}+\widetilde{\zeta} \mathbf{k} \times \overline{\mathbf{u}}\right)+f \mathbf{k} \times h \overline{\mathbf{u}}\right) \cdot \mathbf{d} \mathbf{s} \\
& =\oint\left(\frac{h}{2} \nabla|\overline{\mathbf{u}}|^{2}+(f+\widetilde{\zeta}) \mathbf{k} \times h \overline{\mathbf{u}}\right) \cdot \mathbf{d} \mathbf{s}=\oint \frac{h}{2} \nabla|\overline{\mathbf{u}}|^{2} \cdot \mathbf{d} \mathbf{s}+\int(f+\widetilde{\zeta}) h \overline{\mathbf{u}} \cdot \mathbf{d} \mathbf{n} .
\end{aligned}
$$

The contour integral of the Leibniz pressure terms in equation 2.5 is

$\frac{1}{\rho_{0}} \oint p^{h} \nabla h \cdot \mathbf{d} \mathbf{s}=\frac{1}{\rho_{0}} \iint \nabla \times\left(p^{h} \nabla h\right) d A=\frac{1}{\rho_{0}} \iint\left(\nabla p^{h} \times \nabla h\right) \cdot \mathbf{d} \mathbf{A}=\frac{1}{\rho_{0}} \iint J\left(p^{h}, h\right) d A$,

where $J\left(p^{h}, h\right)$ is the bottom pressure torque, torques that result from variations in pressure along isobaths. Jackson et al. (2006) shows that bottom pressure torques can also be interpreted as geostrophic flow at the seafloor since

$$
J\left(p^{H}, H\right)=\rho f \mathbf{u}^{H}=-\rho f w^{H}
$$

where $H$ is the full ocean depth. This relation follows from the geostrophic relation and by assuming that bottom flows follow topography. Jackson et al. (2006) also notes that when friction is allowed, this condition holds at the top of the bottom Ekman layer, so that bottom pressure torques may act as a communicator between wind stress torque and bottom stress torque, or can be thought of as a parameterization of frictional effects.

The contour integral of equation 2.5 can be written out as

$$
\begin{gathered}
\oint\left(h \overline{\mathbf{u}}_{t}+\frac{h}{2} \nabla|\overline{\mathbf{u}}|^{2}\right) \cdot \mathbf{d} \mathbf{s}+\int(f+\widetilde{\zeta}) h \overline{\mathbf{u}} \cdot \mathbf{d} \mathbf{n}+\oint\left(\nabla \cdot\left(h \overline{\mathbf{u}^{\prime} \mathbf{u}^{\prime}}\right)+\omega\left(\overline{\mathbf{u}}-\mathbf{u}^{h}\right)\right) \cdot \mathbf{d} \mathbf{s} \\
+\frac{1}{\rho_{0}} \iint J\left(p^{h}, h\right) d A-\oint \frac{1}{\rho_{0}}\left(\boldsymbol{\tau}^{0}-\boldsymbol{\tau}^{h}\right) \cdot \mathbf{d} \mathbf{s}=0 .
\end{gathered}
$$

In the special case of a layer of homogeneous density, equation 2.11 simplifies to

$$
\oint\left(h \mathbf{u}_{t}+\frac{h}{2} \nabla|\mathbf{u}|^{2}\right) \cdot \mathbf{d} \mathbf{s}+\int(f+\zeta) h \mathbf{u} \cdot \mathbf{d} \mathbf{n}+\frac{1}{\rho_{0}} \iint J\left(p^{h}, h\right) d A-\oint \frac{1}{\rho_{0}}\left(\boldsymbol{\tau}^{0}-\boldsymbol{\tau}^{h}\right) \cdot \mathbf{d} \mathbf{s}=0
$$

Rearranging the time derivative and vorticity flux terms in equation 2.12 facilitates comparison with equation 2.10:

$$
\oint h \mathbf{u}_{t} \cdot \mathbf{d} \mathbf{s}+\int(f+\zeta) h \mathbf{u} \cdot \mathbf{d} \mathbf{n}=\iint\left(\nabla \times\left(h \mathbf{u}_{t}\right)+\nabla \cdot(f+\zeta) h \mathbf{u}\right) d A
$$

but this can only be re-written as a flux equation for vorticity if the integral is taken over the full water column, so that the bottom boundary is not changing in time, i.e. if we replace $h$ by $H$, which is the ocean floor bathymetry, so that $H_{t}=0$. In this special case, 2.13 can 
be written in flux form as

$$
=\iint\left(((f+\zeta) H)_{t}+\nabla \cdot(f+\zeta) H \mathbf{u}\right) d A
$$

where we have also used that $f_{t}=0$. The result is a flux equation for the vertically-integrated vorticity of the full, barotropic water column.

The relationship between this vorticity equation for the vertically-integrated flow and the vorticity equation for the vertically-averaged flow can be clarified further by the relationship between their pressure gradient terms, bottom pressure torques and JEBAR respectively. As shown in Mertz and Wright (1992),

$$
\frac{1}{\rho_{0}} \nabla \times\left(p^{h} \nabla h\right)=\frac{1}{\rho_{0}} \nabla \times(\bar{p} \nabla h)-\nabla \times\left(\frac{\chi \nabla h}{h}\right),
$$

where the term on the left is proportional to the bottom pressure torque term and the second term on the right is proportional to the JEBAR term. This equation highlights that bottom pressure torques are given by the difference between the torque associated with the vertically-averaged pressure and JEBAR. In a homogeneous layer, the bottom pressure torques are equivalent to the torque associated with the vertically-averaged pressure and the JEBAR term is zero, as we showed in the previous subsection. Equivalently, JEBAR corrects for the fact that the bottom velocity, not the depth-averaged velocity, leads to vortex stretching (Cane et al., 1998).

As explained in Jackson et al. (2006), in our framework, which reduces to a budget of vertically-integrated shallow-water $\mathrm{PV}$ in the homogeneous limit, the role of topography is distributed amongst terms. In a PV budget, the characteristics are contours of $f / h$ and in the barotropic vorticity budget, the characteristics are contours of $f$. In the barotropic vorticity budget, bottom pressure torques force flow across $f$ contours so that it follows $f / h$ contours (Yeager, 2015); topography can allow flow across $f$ contours, but not PV contours. The primary balance that emerges in a barotropic vorticity budget is the partitioning of PV conservation into its planetary vorticity and layer thickness components, whereas PV budgets highlight processes which change the PV of a fluid.

The depth-integrated and depth-averaged frameworks we describe each come with their own set of assumptions. In the depth-integrated framework, the effect of topography on the vorticity budget is highlighted through the bottom pressure torque term. In the depthaveraged framework, the effect of stratification in adjusting to topography is highlighted through the JEBAR term. It goes without saying that the meaning of the budget quantity must be considered in choosing a budget framework. We focus on the vorticity of the depthaveraged velocity because it is analogous to PV, but this choice comes with its own caveats, such as the sensitivity of multiple budget terms on the budget layer depth. 


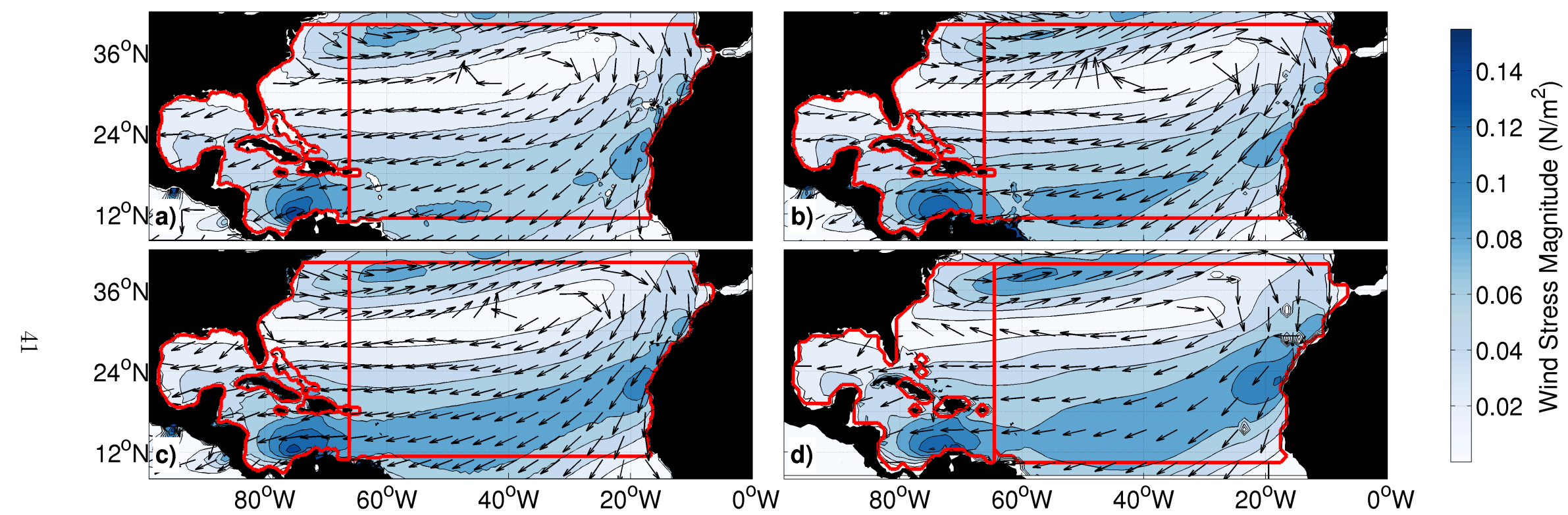

Figure 2-3: Average wind stress in the regions of interest from four different products. Color reflects the wind stress magnitude and arrows show direction. Contour lines are every $0.02 \mathrm{~N} \mathrm{~m}^{-2}$. a) Scatterometer Climatology of Winds. b) NASA's Modern-Era Retrospective Analysis for Research and Applications. c) ECMF's ERA-Interim Reanalysis. d) ECCO state estimate. a-d) The red lines are the contours on which the wind stress forcing terms are calculated, they are defined by the outermost grid point in the a-c) SCOW and d) ECCO products. An arrow is plotted on every 15 data points for panels $\mathbf{a}$ ) and $\mathbf{b}$ ), and every 5 data points for panels $\mathbf{c}$ ) and $\mathbf{d}$ ). 


\subsection{Wind Stress Forcing}

The westerlies and trade winds create a dominant anticyclonic pattern of wind stress curl over the subtropics, which corresponds to a source of negative vorticity for the subtropical North Atlantic. As part of the vorticity budget for the volume of interest, we quantify the wind stress forcing to the west of the A22 cruise track from a variety of wind stress products. To place this number in context, we also calculate the wind stress forcing to the east of the A22 cruise track; this gives an order of magnitude for the wind stress forcing over the entire subtropical gyre. Note that the intention is not to build a budget in the eastern region, but to provide a point of reference. The northern and southern boundaries of the eastern region are at $11^{\circ} \mathrm{N}$ and $40^{\circ} \mathrm{N}$ for all data products, as shown in Figures 2-2 and 2-3.

As derived in Section 2.3, the wind stress contribution to the vorticity budget is

$$
\oint \frac{\tau^{0}}{\rho_{0} h} \cdot \mathbf{d} \mathbf{s}
$$

where $\tau^{0}$ is the wind stress at the surface of the ocean, $z=0, \rho_{0}$ is a constant reference density, $1027 \mathrm{~kg} \mathrm{~m}^{-3}$, and $h$ is the layer thickness from the air-sea interface to the isopycnal that bounds the study volume from below. Taking the contour integral means that we need to know the depth of the isopycnal on the contour. For this, we use the average isopycnal depth from the Gouretski and Koltermann (2004) climatology, depicted in Figure 2-2a. The layer thickness does not vanish on the contour; the coastal points are defined as the shallowest bin where wind stress is estimated.

The wind stress products we analyze are the Scatterometer Climatology of Ocean Winds (SCOW), NASA's Modern-Era Retrospective Analysis for Research and Applications (MERRA) and the European Centre for Medium Range Weather Forecast's (ECMWF) interim reanalysis (ERA). SCOW is based on 10 years of QuikSCAT scatterometer observations. It has been smoothed using harmonic analysis in an attempt to remove extreme events and is said to represent the long term mean state (Risien and Chelton, 2008). SCOW provides data on a $0.25^{\circ}$ grid. MERRA and ERA-interim are satellite era (from 1979 onwards) re-analyses and have $0.6^{\circ} \times 0.5^{\circ}$ and $0.7^{\circ}$ resolution respectively (Rienecker et al., 2011; ECMWF, 2012; Dee et al., 2011). We take the time average of the MERRA and ERA wind stress products over the QuikSCAT era (September 1999-October 2009) to facilitate comparison.

To calculate the wind stress forcing from these wind stress products, we integrate around the contours defined by the outermost grid point in the SCOW product, shown in Figures 2-2a and 2-3a-c, integrating the wind stress anticyclonically around the approximate cruise track and coastlines and cylonically around the Caribbean islands to the west of the cruise track. We interpolate the climatological isopycnal depth to this same contour. The timeaveraged wind stress over the subtropical North Atlantic, shown in Figure 2-3, has the same broad-scale features regardless of the data product. There is generally an anticyclonic 
NASA MERRA, ERA-Interim and
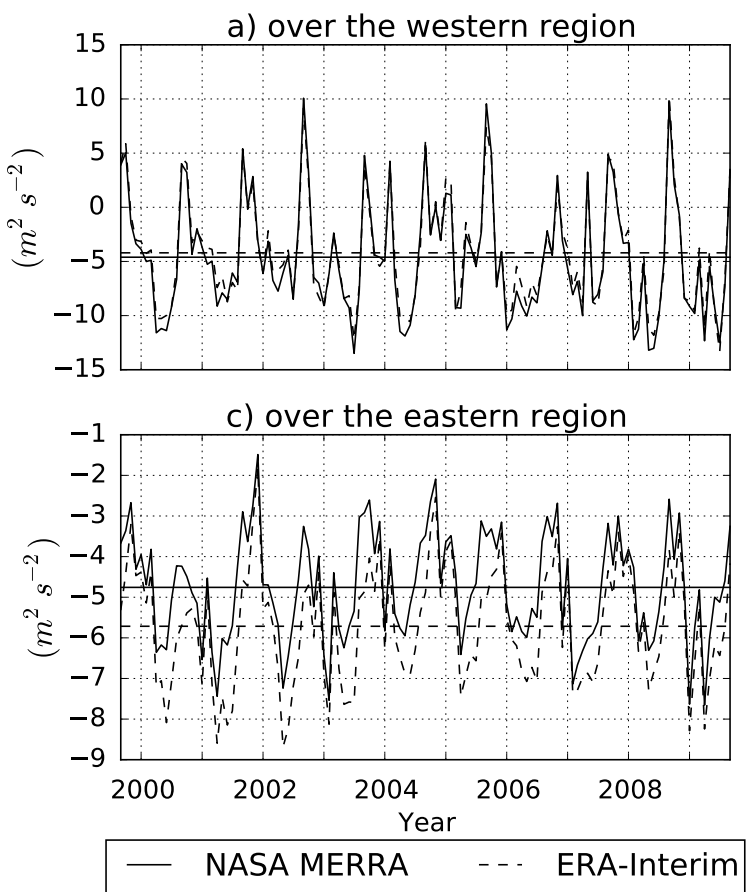

SCOW wind stress forcing

b) over the western region

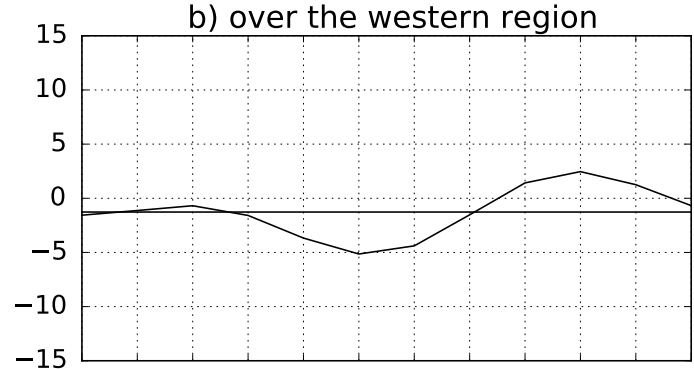

d) over the eastern region

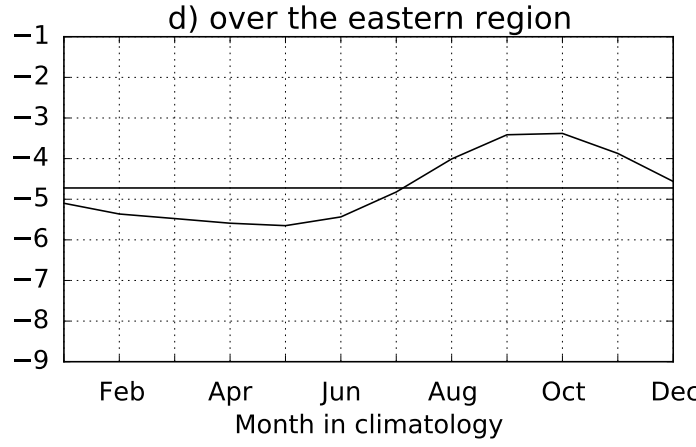

Figure 2-4: Time series of wind stress forcing budget term for (a, c) NASA-MERRA (solid line) and ERA-Interim (dashed line) data products, in the left column and $(\mathbf{b}, \mathbf{d})$ the SCOW climatology, in the right column. The top row $(\mathbf{a}, \mathbf{b})$ displays wind stress forcing time series over the western region, and the bottom row $(\mathbf{c}, \mathbf{d})$ over the eastern region, as shown in Figures 2-2a and 2-3a-c. The straight horizontal lines in each plot highlight the mean wind stress forcing value.

pattern of wind stress over the subtropical gyre, with localized regions of high wind stress in the southern Caribbean and eastern North Atlantic. The products differ in the details of their smaller scale features.

We calculate the wind stress forcing in the ECCO state estimate using a contour defined by the outermost grid point in the ECCO product, shown in Figures 2-2b and 2-3d, and the depth of the $\sigma_{0}=27.15 \mathrm{~kg} \mathrm{~m}^{-3}$ potential density anomaly in the ECCO model, as shown in Figure 2-2b.

The wind stress forcing results shown in Figure 2-4 confirm that the wind is generally a negative source of vorticity in the subtropical gyre. A seasonal cycle is apparent with the most negative wind stress forcing occurring early in the year. The wind stress forcing over the western region has higher variability, likely due to the fact that the western region is smaller than the eastern region and that the majority of the western contour is coastal while the eastern contour includes more deep ocean length.

The estimates of mean wind stress forcing, also quoted in Table 2-1, in the western region vary, ranging from $-1.3 \pm 1.3 \mathrm{~m}^{2} \mathrm{~s}^{-2}$ in the SCOW product, to $-4.6 \pm 1.0 \mathrm{~m}^{2} \mathrm{~s}^{-2}$ in MERRA and $-4.2 \pm 0.9 \mathrm{~m}^{2} \mathrm{~s}^{-2}$ in ERA, reported here with $95 \%$ confidence intervals. The mean 

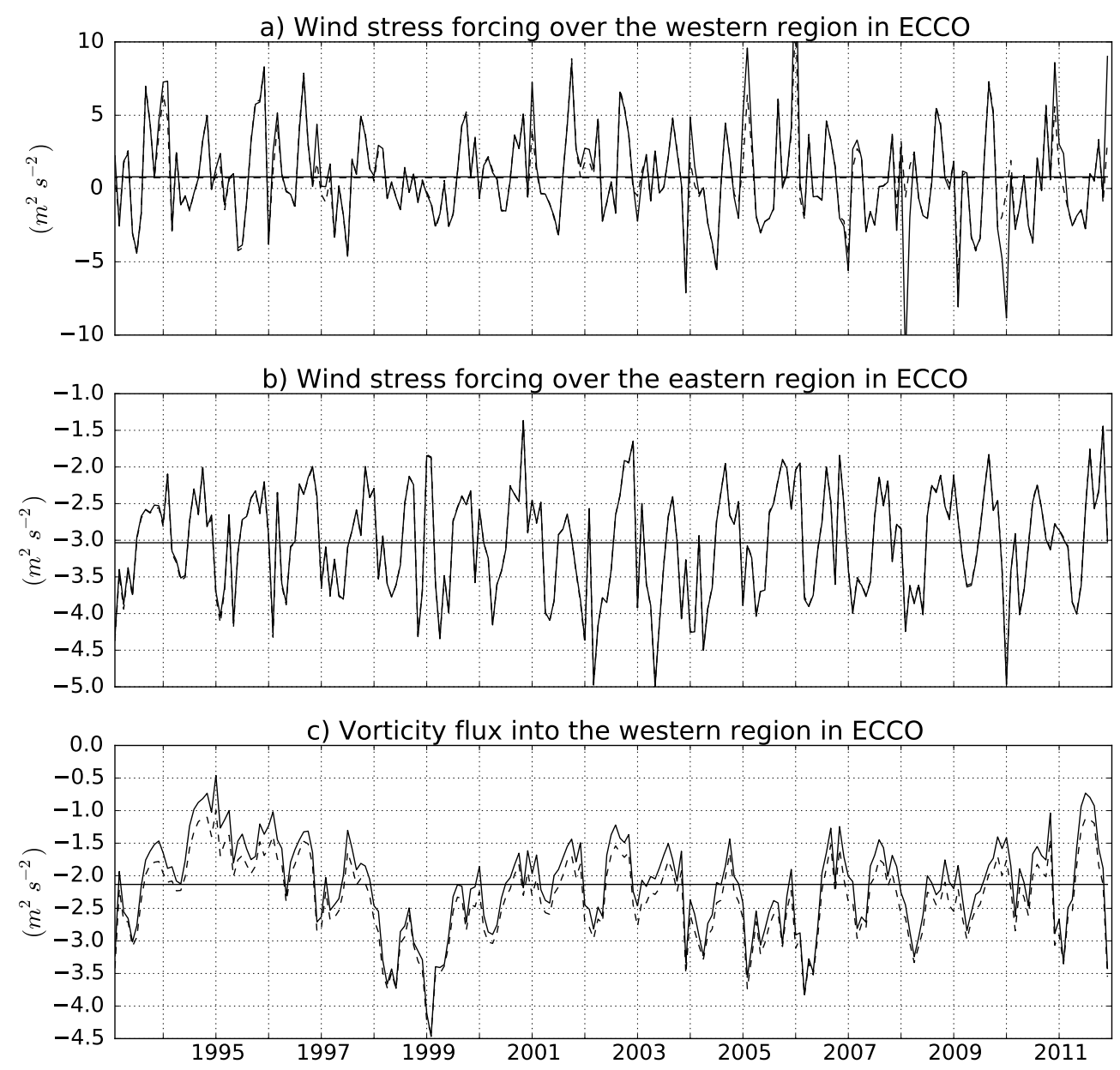

Figure 2-5: Time series (monthly averages from 1992-2010) of the wind stress forcing and vorticity flux terms in the ECCO model west of $64.5^{\circ} \mathrm{W}$ in the North Atlantic. a) Time series of the wind stress forcing budget term for the western region, $\mathbf{b}$ ) wind stress forcing budget term for the eastern region and c) the advective vorticity flux term for the western region. The dashed lines in a) and b) are wind stress forcing values calculated from ECCO mean layer thickness instead of the layer thickness at each time step. The dashed line is indistinguishable from the solid line in $\mathbf{b}$ ). The dashed line in c) is the vorticity flux that results if each month is corrected to be in mass balance. The full time mean of each term is depicted by a straight line through the time series; their magnitudes are listed in Table 2-1. 
wind stress forcing in the eastern region is more robustly negative, as one would expect given the dominant anticyclonic winds. The mean wind stress forcing over the eastern region in SCOW is $-4.7 \pm 0.5 \mathrm{~m}^{2} \mathrm{~s}^{-2}$, the MERRA value is comparable at $-4.8 \pm 0.2 \mathrm{~m}^{2} \mathrm{~s}^{-2}$ and the ERA value is $-5.7 \pm 0.3 \mathrm{~m}^{2} \mathrm{~s}^{-2}$.

Coastal winds are difficult to measure with satellite scatterometers (Yu and Jin, 2012), and this may be a drawback for this application of the SCOW product. The MERRA and ERA wind stress forcing agree well in the western region, and though their variability is similar in the eastern region, there is a systematic bias between them (Figure 2-4).

It is unclear which dataset provides the most relevant value and their spread reflects the degree of uncertainty that exists. The reported $95 \%$ confidence intervals were evaluated using a bootstrap method with 10,000 bootstrap samples (Efron and Gong, 1983). We use this technique to calculate confidence intervals throughout. Note that the SCOW record is a climatology based on the same ten years that are shown for the MERRA and ERA records, but provides just one seasonal cycle. We use the degrees of freedom in the seasonal cycle only, so that we likely underestimate the degrees of freedom in the data and hence overestimate the confidence intervals in our calculation from the SCOW data. There is also uncertainty from the climatological layer thickness product embedded in our result.

The wind stress in the ECCO model is based on QuikScat data where available and climatological QuikScat data otherwise, but is adjusted to fit ocean observations within ECCO's dynamically consistent framework (Wunsch and Heimbach, 2013a). The mean wind stress sources of vorticity in the ECCO model are $0.8 \pm 0.4 \mathrm{~m}^{2} \mathrm{~s}^{-2}$ and $-3.0 \pm 0.1 \mathrm{~m}^{2} \mathrm{~s}^{-2}$ for the western and eastern regions respectively, reported again with $95 \%$ confidence intervals. The full time series, displayed in Figure 2-5, shows a seasonal cycle similar to that in the other data products, with a peak occurring early in the year. The wind stress forcing over the western region is as variable as the MERRA and ERA products, but its mean is robustly positive. This could be a product of the adjustments performed in the ECCO model to fit ocean observations in a dynamically consistent way. Indeed, the positive wind stress forcing over the western region contributes to closing the vorticity budget in ECCO, as discussed in Section 2.6.

We also show, in the dashed lines, the wind stress forcing in ECCO calculated using the mean layer thickness over the full ECCO time series instead of the layer thickness at each time step. The two time series do not differ significantly, especially in the eastern region, where the difference between the lines is not perceptible and the wind stress forcing means are the same. In the western region, the mean wind stress forcing calculated using the mean layer thickness is $0.7 \pm 0.4 \mathrm{~m}^{2} \mathrm{~s}^{-2}$. The agreement between the two calculations indicates that seasonal variation in layer thickness does not influence the wind stress forcing term as much as wind stress variability does, so that it is reasonable to calculate wind stress forcing from the observational data products using a mean climatological layer thickness. 


\begin{tabular}{|l|l|l|}
\hline Vorticity budget term & Data product & $\left(m^{2} s^{-2}\right)$ \\
\hline Wind stress forcing & & \\
western region & SCOW & $-1.3 \pm 1.3$ \\
& NASA MERRA & $-4.6 \pm 1.0$ \\
& ERA-Interim & $-4.2 \pm 0.9$ \\
& ECCO & $0.8 \pm 0.4$ \\
\cline { 2 - 3 } eastern region & SCOW & $-4.7 \pm 0.5$ \\
& NASA MERRA & $-4.8 \pm 0.2$ \\
& ERA-Interim & $-5.7 \pm 0.3$ \\
& ECCO & $-3.0 \pm 0.1$ \\
\hline Advective vorticity flux & A22 2003 & $-2.4 \pm 2.5$ \\
& A22 2012 & $-5.3 \pm 2.2$ \\
& ECCO & $-2.1 \pm 0.1$ \\
\hline
\end{tabular}

Table 2-1: Summary of wind stress forcing and advective vorticity flux budget terms in both observations and in the ECCO state estimate. The wind stress forcing is calculated over the western and eastern regions depicted in Figure 2-3. The reported spread on all wind stress forcing and ECCO terms are $95 \%$ confidence intervals, the reported spread on the A22 vorticity flux terms are from a random error propagated through the calculation. ECCO wind stress and advective vorticity flux time series are shown in Figure 2-5. Remaining ECCO budget terms are shown in Figure 2-8 and their time means are reported in Table 2-2.

These wind stress forcing results set the stage for comparison with the advective vorticity flux.

\subsection{Advective vorticity flux}

The advective flux of vorticity out of the western study volume gives an integrated measure of vorticity sources in the western subtropical gyre. The advective vorticity flux is given by the depth-averaged velocity normal to the bounding contour multiplied by the absolute vorticity (planetary and relative vorticity) of the depth-averaged velocity on the contour,

$$
-\int(f+\widetilde{\zeta}) \overline{\mathbf{u}} \cdot \mathbf{d} \mathbf{n}=-\int\left(\frac{f+\widetilde{\zeta}}{h}\right) \mathbf{U} \cdot \mathbf{d} \mathbf{n}
$$

where $U$ is the depth-integrated velocity. For a layer with homogeneous density, this is equivalent to the depth-integrated PV flux. We calculate the advective vorticity flux across two occupations of the A22 section (Figure 2-1), done in October of 2003 and March of 2012 using CTD (Conductivity Temperature Depth) density, lowered ADCP (Acoustic Doppler Current Profiler) and shipboard ADCP velocity data. Note that we can only calculate 
the contribution of along-track variations of velocity to the relative vorticity from the section. The A22 section is part of the WOCE/CLIVAR observing effort, with CTD data and lowered ADCP data available at http://cchdo.ucsd.edu/ and shipboard ADCP data at http://ilikai.soest.hawaii.edu/sadcp/.

The CTD data are used to identify the $\sigma_{0}=27 \mathrm{~kg} \mathrm{~m}^{-3}$ potential density anomaly surface that bounds the study volume from below. As can be seen in Figure 2-6a, the layer depth profile is very similar from one occupation to the next. In general, the layer gradually deepens from south to north, due to the downwelling associated with the subtropical gyre, and shoals dramatically across the Gulf Stream, which is geostrophic to first order. The layer shoals at $18^{\circ} \mathrm{N}$ because the water depth is shallow in the vicinity of Puerto Rico.

We combine the shipboard and lowered ADCP data to calculate the velocity perpendicular to the section, shown in Figure 2-6b. The lowered ADCP data, available at CTD station locations, are used only to fill in velocities shallower than $30 \mathrm{db}$, which is the shallowest bin available from shipboard ADCP measurements. This is done in order to account for Ekman flux in the surface layer. Most of the velocity data is from the finer along-track resolution shipboard ADCP data. Using only velocity data from the shipboard ADCP and extrapolating uniformly to the surface yields vorticity flux values with the same integer value, indicating that the Ekman flux does not contribute significantly to the vorticity flux across this layer; however, we include the lowered ADCP data for completeness. We depth average the velocity shallower than the $\sigma_{0}=27 \mathrm{~kg} \mathrm{~m}^{-3}$ isopycnal. We then bin average the layer depth and velocity by averaging all data within $0.05^{\circ}$ of latitude bins of each point on a $0.025^{\circ}$ grid to dampen ageostrophic motions and instrumental noise while still resolving the width of the Gulf Stream. 
a) Isopycnal Depth, h $(m)$

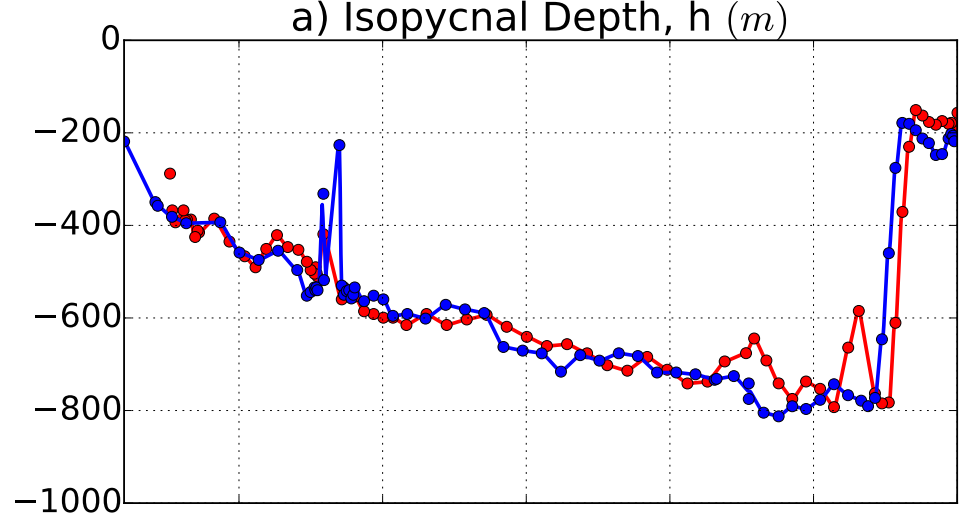

c) Vorticities, $f$ and $\zeta\left(s^{-1}\right)$

$\stackrel{\infty}{\infty}$

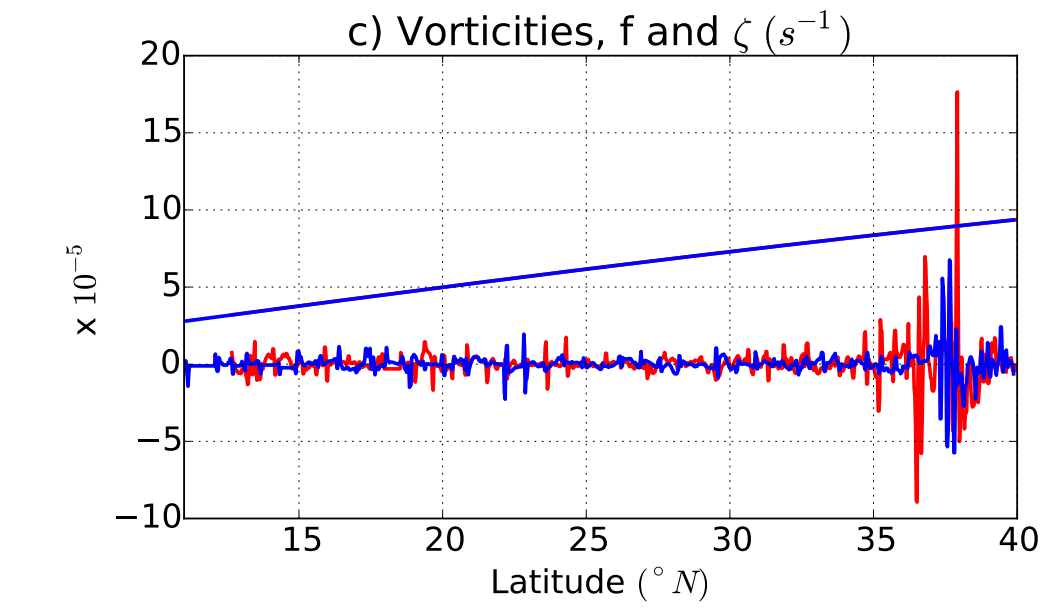

b) Perpendicular Velocity, $\mathrm{u}\left(\mathrm{ms}^{-1}\right)$

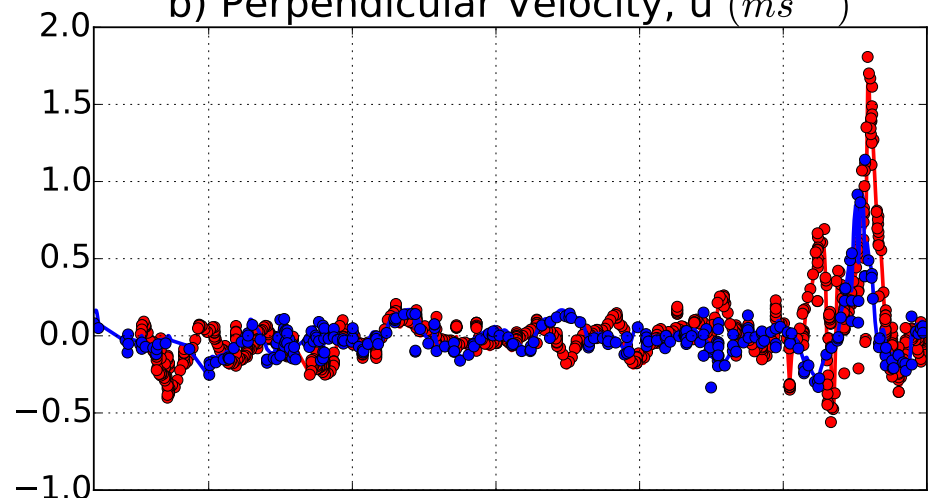

d) Cumulative Vorticity Flux $\left(m^{2} s^{-2}\right)$

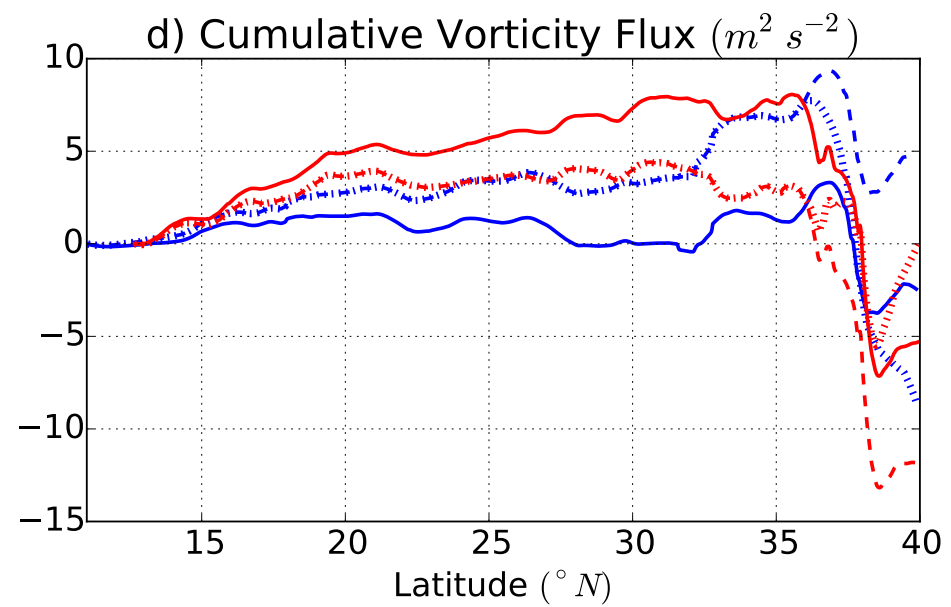

Figure 2-6: Components of calculation of vorticity flux across the A22 cruise track shown in Figure 2-1. WOCE/CLIVAR A22 2003 occupation data are in blue, 2012 in red. a) Depth of $\sigma_{0}=27 \mathrm{~kg} \mathrm{~m}^{-3}$ isopycnal along A22 track from CTD data. Dots indicate CTD data points, lines indicate data averaged into 0.05 degrees of latitude bins. b) Velocity perpendicular to the A22 cruise track averaged from the $\sigma_{0}=27 \mathrm{~kg} \mathrm{~m} \mathrm{~m}^{-3}$ isopycnal to the surface. Dots indicate ADCP data points, lines indicate data averaged into $0.05^{\circ}$ latitude bins. c) Vorticity along A22 track from bin and depth-averaged velocity data. The planetary vorticity, $f$, increases monotonically with latitude and the relative velocity, $\zeta$, is generally smaller and noisy. d) Solid lines are the cumulative vorticity flux across A22, which is the product of b) and the sum of $\mathbf{c}$ ) integrated from the south. Dashed lines are the cumulative vorticity flux for the velocities without a mass balance correction. Dotted lines are the cumulative vorticity flux with a mass balance correction applied only to data points in the Gulf Stream region. 
We apply a mass balance correction to the velocity, adding or subtracting a constant velocity to all bin averaged data points to force the across-track mass flux to zero. While the layer may be out of mass balance instantaneously, in this study we are not interested in the transient processes that would cause that, but want to characterize the time average flow field. The absolute correction that we apply is $-0.028 \mathrm{~m} \mathrm{~s}^{-1}$ to the 2003 dataset and $+0.030 \mathrm{~m} \mathrm{~s}^{-1}$ to the 2012 dataset. Alternatively, we applied the mass balance correction by adding a constant velocity to all bin averaged points in the Gulf Stream region only (North of $36^{\circ} \mathrm{N}$ ), where the largest errors may be expected to occur. In this case the corrections are very large, $-0.30 \mathrm{~m} \mathrm{~s}^{-1}$ and $+0.25 \mathrm{~m} \mathrm{~s}^{-1}$ in 2003 and 2012 respectively; these values are well above the $0.01 \mathrm{~m} \mathrm{~s}^{-1}$ uncertainty in our velocity measurements. Even if these mass imbalances were compensated for by diapycnal velocity, the "entrainment stress" term would still be one or two orders of magnitude smaller than the vorticity flux, as discussed in Section 2.6.

We calculate the total advective flux of vorticity across the track by multiplying the across-track depth-averaged velocity with the vorticity of the depth-averaged velocity and integrating along the track. Across-track velocity is defined as positive out of and negative into of the western region. We assume that the perpendicular velocity into the land boundaries is zero. The product of the across-track velocity and the vorticity is dominated by the large positive velocities of the Gulf Stream in the north, which are multiplied by a larger planetary vorticity than the compensating negative velocities to the south, resulting in a flux out of the western region, or a net negative advective vorticity flux across the section.

We are only able to calculate the along-track component of the relative vorticity from these observations, but expect that the full relative vorticity is well-approximated in this way as the track runs perpendicular to the Gulf Stream where most lateral variation in velocity occurs. Further, the vorticity of the depth-averaged velocity, $\widetilde{\zeta}=\bar{v}_{x}-\bar{u}_{y}$, makes a negligible contribution to the total advective flux of vorticity as it is only comparable in magnitude to changes in $f$ in the vicinity of the Gulf Stream (see Figure 2-6c). Because $\widetilde{\zeta}$ is opposite-signed on either side of the single-signed current, the contributions from either side of the current tend to cancel each other out in the cumulative vorticity flux.

As shown in Figure 2-6d, the total advective vorticity flux comes out to $-2.4 \pm 2.5 \mathrm{~m}^{2} \mathrm{~s}^{-2}$ and $-5.3 \pm 2.2 \mathrm{~m}^{2} \mathrm{~s}^{-2}$ for the 2003 and 2012 occupations respectively, as shown by the solid lines in Figure 2-6d. The uncertainty estimates reported are arrived at by propagating an error of $0.01 \mathrm{~m} \mathrm{~s}^{-1}$ in the velocity through the calculation. The dashed lines in Figure 2-6d are the across-track vorticity flux before velocities are corrected for mass balance, yielding +4.6 and $-11.8 \mathrm{~m}^{2} \mathrm{~s}^{-2}$ for the 2003 and 2012 occupations respectively. The dotted lines are the across-track vorticity flux with a mass balance applied to the Gulf Stream region only, giving -8.6 and $0.03 \mathrm{~m}^{2} \mathrm{~s}^{-2}$, for the 2003 and 2012 occupations respectively. The advective vorticity flux is very sensitive to the degree of mass balance across the section. 
Without applying the mass balance condition, most of the vorticity flux across these sections is associated with a layer mass flux across the section.

The fact that the advective vorticity flux is negative, together with the negative or small wind stress forcing found in Section 2.4, implies that there is a positive source contributing to the vorticity budget in the western region. Friction would provide such a source, as in the Stommel and Munk models of gyre circulation, for example. Furthermore, these advective vorticity fluxes are on the same order as the wind stress forcing values calculated over the eastern region (terms summarized in Table 2-1), which is also consistent with Stommel and Munk models, in which the negative wind stress forcing over the interior is balanced by friction in the west. Because we do not quantify the magnitude of the remaining terms in the budget from observations, we cannot establish conclusively that friction will be a sizable term in the real ocean, only that it is the most likely term to close the budget. The magnitude of the remaining terms can be diagnosed from the ECCO state estimate and this is discussed further in Section 2.6.

If the gyre dynamics were primarily inertial, the advective vorticity flux would be very small compared to the other terms in the budget as vorticity would be conserved along streamlines. As the vorticity flux is on the same order as the wind stress forcing over the subtropical gyre, inertia does not appear to dominate at first glance.

The relative size of the integrated advective vorticity flux versus its cumulative absolute value (i.e. $\left.\int_{0}^{L_{y}}|u(f+\zeta)| d y\right)$ gives a measure of how inertial the large-scale dynamics are regardless of the size of the other terms in the budget. In a primarily inertial balance, the net vorticity flux would be much smaller than its cumulative absolute value, as vorticity is conserved along inertial streamlines. The cumulative absolute vorticity fluxes are $21 \mathrm{~m}^{2} \mathrm{~s}^{-2}$ and $33 \mathrm{~m}^{2} \mathrm{~s}^{-2}$ for the 2003 and 2012 data respectively, which are one order of magnitude larger than the vorticity fluxes we calculate $\left(-2.4 \pm 2.5 \mathrm{~m}^{2} \mathrm{~s}^{-2}\right.$ and $-5.3 \pm 2.2 \mathrm{~m}^{2} \mathrm{~s}^{-2}$.) The cumulative absolute vorticity flux is much larger than the vorticity flux because the contributions from the relative vorticity component of the vorticity flux no longer cancel out on either side of the current, but compound. Hence, from this perspective, there is a significant inertial component in the advective vorticity flux. The inertial component of the flow has zero vorticity flux associated with it, so that the non-inertial component of the balance is highlighted in our framework. This does not mean that the inertial component is not significant, just that something akin to friction is necessary to close the system. As highlighted in Vallis (2006), even a dominantly inertial forced system requires friction in order to achieve a steady-state balance.

We calculate the advective vorticity fluxes in the ECCO state estimate across a boundary similar to the A22 cruise track (Figure 2-1), along $64.5^{\circ} \mathrm{W}$ and $40^{\circ} \mathrm{N}$ and present the results in Figure 2-7 in the same format as the A22 results are presented in Figure 2-6. Notable differences between the A22 and ECCO data are that the Gulf Stream is much wider and 
slower in ECCO due to its $1^{\circ}$ resolution, resulting in negligible relative vorticity. However, the Gulf Stream volume transport in ECCO is well matched to observations. The mean mass flux into the study volume in ECCO is zero, and if we apply mass balance at each time step in the same way as we did for the A22 data, the ECCO vorticity flux is not significantly altered, as shown by the dashed line in Figure 2-5. Instead of two occupations, ECCO provides 19 years of monthly data that reveal significant variability in the integrated vorticity flux. Though the vorticity flux calculated from A22 sections falls within the range of values calculated in the ECCO model, this calls the representative nature of each individual A22 section into question and emphasizes that our calculations from observations can only give an order of magnitude estimate. The ECCO vorticity flux has a distinct seasonal cycle that is associated with spinning up and down of the gyre. The mean vorticity flux calculated across $64.5^{\circ} \mathrm{W}$ in the ECCO model is $-2.1 \pm 0.1 \mathrm{~m}^{2} \mathrm{~s}^{-2}$, quoted here with a 95\% confidence interval found using the bootstrap method as before. The mean integrated vorticity flux in ECCO is less than a quarter of its cumulative absolute value, $8.6 \mathrm{~m}^{2} \mathrm{~s}^{-2}$, indicating that there is a non-negligible inertial component to the Gulf Stream in ECCO as well, albeit a smaller component than in the observations. This is to be expected based on the $1^{\circ}$ resolution of the ECCO state estimate and resulting broader Gulf Stream. The vorticity flux in ECCO is also on the same order as the mean wind stress forcing over the eastern region in the ECCO model, $-3.0 \pm 0.1 \mathrm{~m}^{2} \mathrm{~s}^{-2}$, consistent with a large-scale balance between wind stress forcing and a positive source of vorticity in the western part of the North Atlantic. 
a) Isopycnal Depth, $\mathrm{h}(m)$

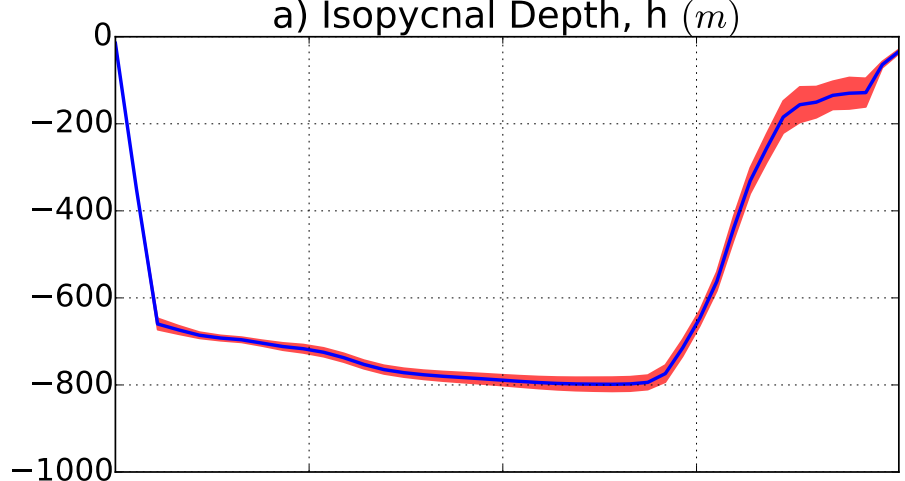

$-1000$

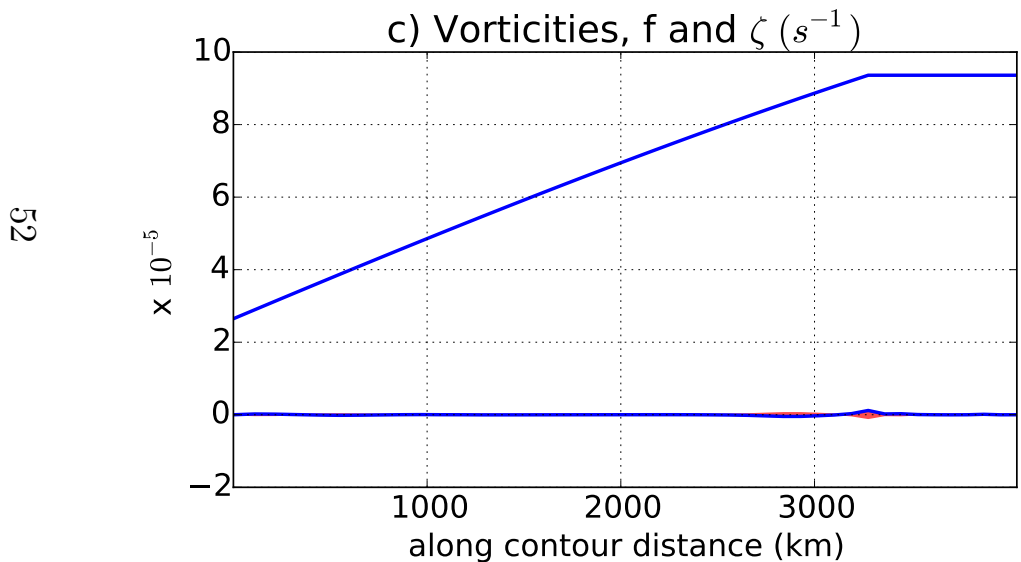

b) Perpendicular Velocity, $\mathrm{u}\left(\mathrm{ms}^{-1}\right)$
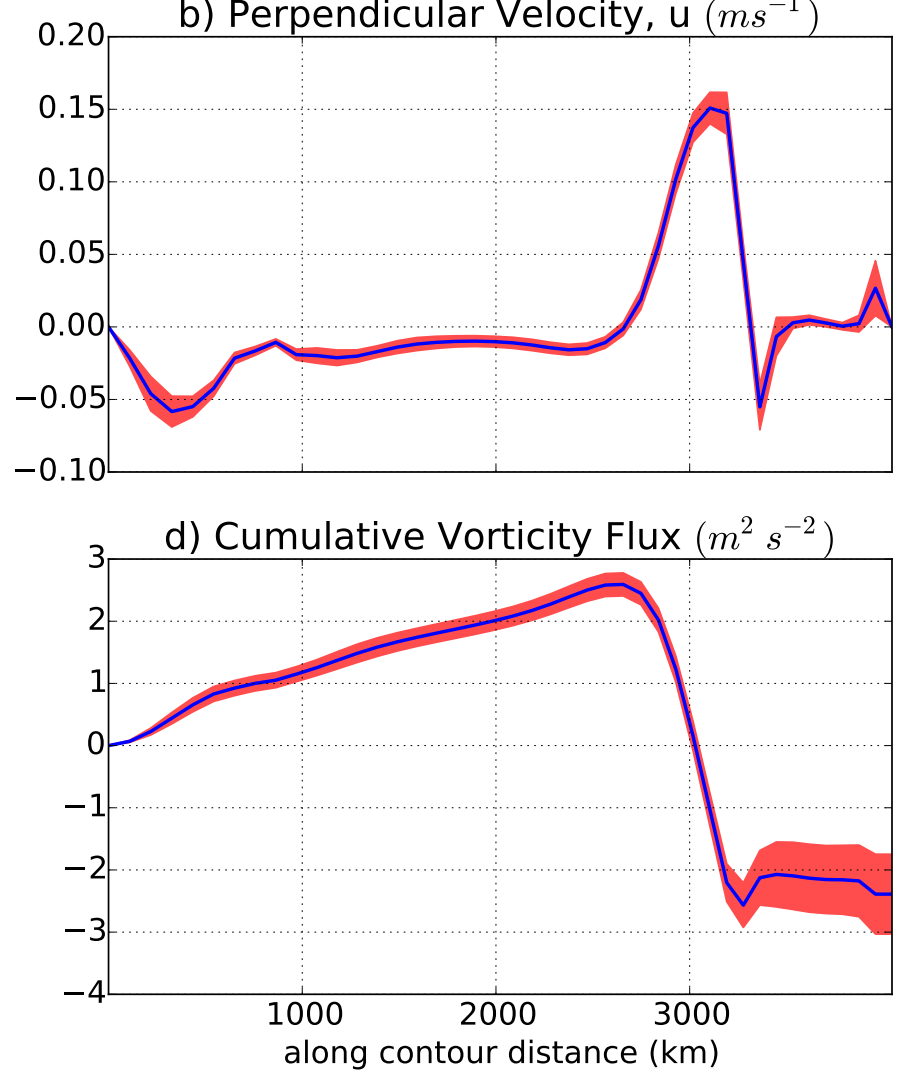

time mean \pm 1 std dev

Figure 2-7: As in Figure 2-6 for the ECCO state estimate analysis. The time mean is in blue, the red envelope shows the spread of one standard deviation from the mean for all ECCO time points (monthly averages from 1992-2010.) a) Depth of $\sigma_{0}=27.15 \mathrm{~kg} \mathrm{~m}^{-3}$ isopycnal along the ECCO boundary (shown in Figure 2-1). b) Zonal velocity across the ECCO boundary averaged from the $\sigma_{0}=27.15 \mathrm{~kg} \mathrm{~m}-3$ isopycnal to the surface. c) Vorticity along the ECCO boundary in the ECCO model. Mean planetary vorticity decreases at the end of the contour because the contour adapts to outcropping. The relative vorticity is much smaller than the planetary vorticity. d) Cumulative vorticity flux across the ECCO boundary, which is the product of $\mathbf{b}$ ) and $\mathbf{c}$ ) integrated from the south. 


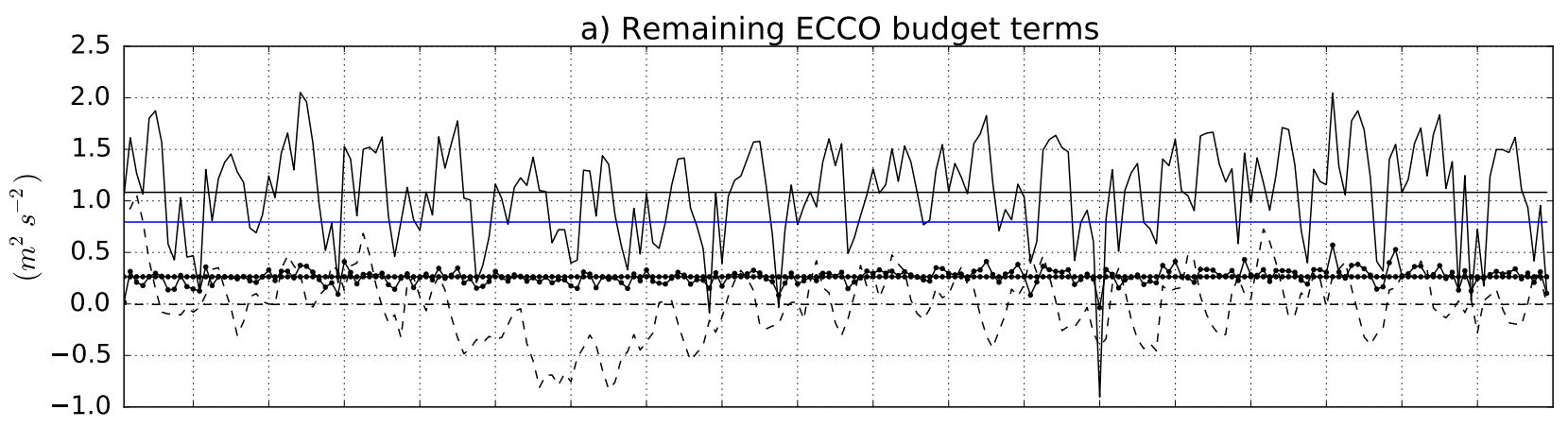

\section{- Lateral friction \\ - Bottom drag \\ - - "JEBAR" term \\ - Mean wind stress forcing}

b) Balance between remaining budget terms and vorticity flux

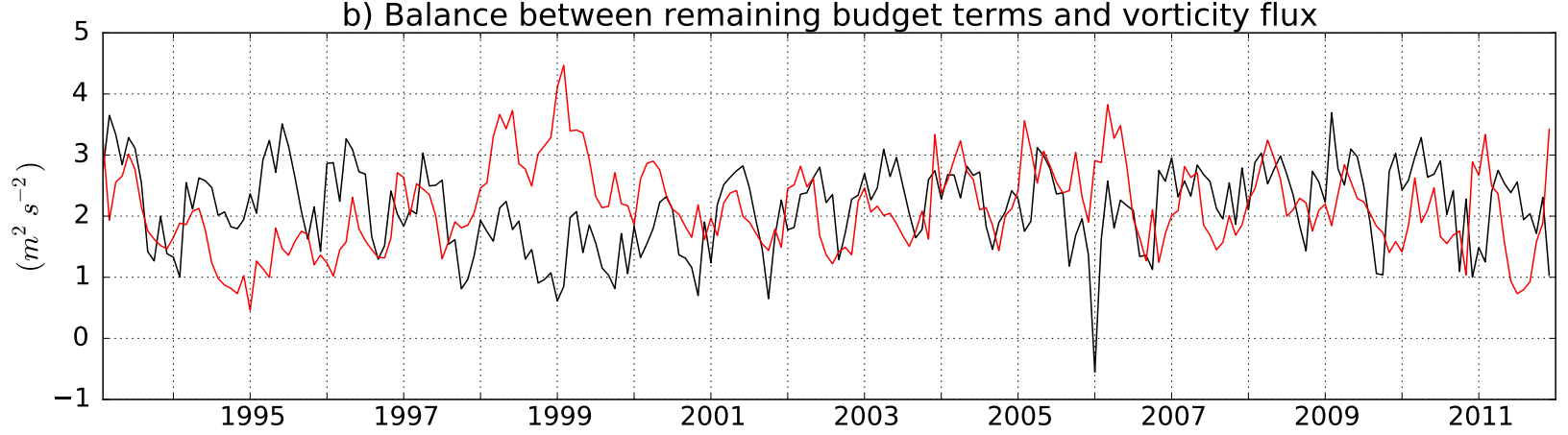

Figure 2-8: Time series (monthly averages from 1992-2010) of the remaining significant terms in the vorticity budget and demonstration of the approximate balance between them and the vorticity flux in the ECCO model west of $64.5^{\circ} \mathrm{W}$ in the North Atlantic. a) Lateral friction, bottom drag and JEBAR term time series are shown in solid, dotted and dashed lines respectively as shown in the legend. The full time mean of each term is depicted by a straight line through the time series. The full time mean of the wind stress forcing term over the western region is also shown for reference; its time series has a much larger range and is shown in Figure 2-5. b) The time series of the sum of the lateral friction, bottom drag, JEBAR and the mean of the wind stress forcing terms is depicted in black and the magnitude of the vorticity flux term in red. All term magnitudes are listed in Table 2-2. 


\subsection{Remaining budget terms}

The remaining terms in the budget of vorticity for the depth-averaged flow can be diagnosed in the ECCO model, and we find that lateral friction and bottom drag provide the remaining sources of positive vorticity to balance the budget in the ECCO model.

The bottom stress is parameterized as $\boldsymbol{\tau}^{h}=\rho_{0} c_{D}\left|\mathbf{u}^{h}\right| \mathbf{u}^{h}$ in the ECCO model, where $c_{D}$ is a bottom drag coefficient and the stress only applies where $h$ corresponds to bathymetry. Thus, the term in the vorticity equation is

$$
-\oint \frac{\tau^{h}}{\rho_{0} h} \cdot \mathbf{d s}=-\oint \frac{c_{D}\left|\mathbf{u}^{h}\right| \mathbf{u}^{h}}{h} \cdot \mathbf{d} \mathbf{s}
$$

Because the bottom velocity is primarily anticyclonic on our contour, this term is robustly positive, with a time mean of $0.26 \pm 0.01 \mathrm{~m}^{2} \mathrm{~s}^{-2}$. As indicated by its small $95 \%$ confidence intervals and the time series shown in Figure 2-8, the contribution from bottom drag is relatively constant.

The laplacian viscosity term,

$$
\oint \overline{A_{h} \nabla^{2} \mathbf{u}} \cdot \mathbf{d} \mathbf{s}
$$

is also positive, as it acts counter to the anticyclonic flow, adjusting it to the no-slip boundary conditions in the ECCO model. This lateral friction term has a time mean contribution of $1.08 \pm 0.06 \mathrm{~m}^{2} \mathrm{~s}^{-2}$, and has a seasonal cycle associated with the spinning up and down of the gyre: with higher values at the beginning of the year, when Gulf Stream velocities are larger. The co-variability of the lateral friction and vorticity flux terms is apparent in Figure $2-8$, though they are not statistically significantly correlated. The viscosity in the ECCO model is parameterizing smaller scale frictional processes, such as eddy decay and internal wave breaking.

Hence, in the ECCO model, there is a primary balance between the negative advective vorticity flux, and positive contributions from lateral friction, bottom drag and wind stress forcing over the western region, as will be discussed further in the final section.

The storage term,

$$
\widetilde{\Gamma}_{t}=(\oint \overline{\mathbf{u}} \cdot \mathbf{d} \mathbf{s})_{t}
$$

can be calculated from the ECCO state estimate, and is found to be a negligible term, on the order of $0.01 \mathrm{~m} \mathrm{~s}^{-1}$, with variability on the order of $0.1 \mathrm{~m} \mathrm{~s}^{-1}$.

The final terms vanish in the limit of homogeneous density, so that they can be thought of as corrections due to the fact that our study volume is stratified. They do not contribute significantly to the budget in the ECCO model and we estimate that they are negligible contributors to the budget from observations. 
The term that accounts for the effects of baroclinicity and interactions with topography,

$$
\iint J\left(\chi, \frac{1}{h}\right) d A
$$

where $\chi=h\left(\bar{p}-p^{h}\right) / \rho_{0}$, is analogous to an integrated "JEBAR" (Joint Effect of Baroclinicity and Relief) term. This analogy only holds directly when the study volume is bounded by topography from below, otherwise, the depth of the isopycnal that bounds our study volume from below is the depth that enters in our term and the analogy does not hold. This term, which can be thought of as a correction for the fact that it is the bottom velocity rather than the depth-averaged velocity that leads to vortex stretching, is depicted in Figure 2-8. Its time average is not distinguishable from zero, $0.01 \pm 0.04 \mathrm{~m}^{2} \mathrm{~s}^{-2}$.

We expect the velocity anomaly term,

$$
\oint \frac{1}{h}\left(\nabla \cdot\left(h \overline{\mathbf{u}^{\prime} \mathbf{u}^{\prime}}\right)\right) \cdot \mathbf{d} \mathbf{s}
$$

to be small compared to the vorticity flux, given that it scales roughly as the relative vorticity contribution to the vorticity flux, which we have shown to be small compared to the planetary vorticity contribution. In the ECCO model, this term is negligible, with a mean of $0.039 \pm 0.001 \mathrm{~m}^{2} \mathrm{~s}^{-2}$. If we make the simplifying assumptions that $\mathbf{u}=0$ along the coast, and that gradients across the track of our observations are much smaller than gradients along the track, then we can estimate the size of this term from observations as

$$
\int \frac{1}{h} \frac{\partial}{\partial s}\left(h \overline{\mathbf{u}^{\mathbf{s}} \mathbf{u}^{\mathbf{s} \prime}}\right) d s
$$

where $\mathbf{u}^{\mathbf{s} \prime}$ is the anomaly from the depth-averaged flow in the along-track direction and $\frac{\partial}{\partial s}$ is the derivative in the along-track direction. Calculating this term from the 2003 and 2012 A22 hydrographic sections yields 0.03 and $0.01 \mathrm{~m}^{2} \mathrm{~s}^{-2}$ respectively, which are two orders of magnitude smaller than the mean vorticity flux. The assumptions required to estimate this term are more stringent than the assumptions made to calculate the mean vorticity flux, but this order of magnitude estimate indicates that this term is likely negligible in observations.

We do not have the diagnostics necessary to estimate the entrainment stress in the ECCO model, but we expect that it is not a significant term in the budget. To get an upper bound estimate of the entrainment stress,

$$
\oint \frac{1}{h}\left(\omega\left(\overline{\mathbf{u}}-\mathbf{u}^{h}\right)\right) \cdot \mathbf{d} \mathbf{s}
$$

from observations, we can assume that the approximately $50 \mathrm{~Sv}$ imbalance across the section is compensated over the $5 \times 10^{12} \mathrm{~m}^{2}$ area of the western region by a diapycnal velocity of $10^{-5} \mathrm{~ms}^{-1}$. A conservative estimate of the layer thickness is $300 \mathrm{~m}$, and of $\left(\mathbf{u}-\mathbf{u}^{-h_{1}}\right)$ 


\begin{tabular}{|l|c|c|}
\hline ECCO vorticity budget term & $\begin{array}{c}\text { Full time mean term } \\
\left(m^{2} s^{-2}\right)\end{array}$ & $\begin{array}{c}\text { Time mean/eddy components } \\
\left(m^{2} s^{-2}\right)\end{array}$ \\
\hline $\begin{array}{l}\text { Wind stress forcing } \\
\text { western region } \\
\text { eastern region }\end{array}$ & $0.8 \pm 0.4$ & $0.7 / 0.1$ \\
\hline Advective vorticity flux & $-3.0 \pm 0.1$ & $-3.0 / 0$ \\
\hline Lateral friction & $-2.1 \pm 0.1$ & $-1.9 /-0.2$ \\
\hline Bottom drag & $1.08 \pm 0.06$ & $1.16 /-0.16$ \\
\hline JEBAR & $0.26 \pm 0.01$ & $0.26 / 0$ \\
\hline Sum of time mean terms & $-0.01 \pm 0.04$ & $-0.03 / 0.024$ \\
\hline
\end{tabular}

Table 2-2: Summary of all time-mean vorticity budget terms in the ECCO state estimate and their mean/eddy breakdown over the western region. The "full time mean term" is the mean of the full time series depicted in Figures 2-5 and 2-8. The reported spread on all terms are 95\% confidence intervals calculated using the bootstrap method. The time mean component of each term is found by using the time mean ECCO fields to calculate the size of each term (e.g. the time mean depthaveraged velocity and layer thickness), and the eddy component is the difference between the full time mean and the contribution from time mean fields.

is $0.1 \mathrm{~ms}^{-1}$. Integrating these estimates together gives an entrainment stress of order $0.01 \mathrm{~m}^{2} \mathrm{~s}^{-2}$, which is also two orders of magnitude smaller than the advective vorticity flux.

\subsection{Conclusions}

In this study, we diagnosed the large-scale balance of vorticity for the Gulf Stream through a vorticity budget for a control volume lying west of the A22 cruise track (nominally along $66^{\circ} \mathrm{W}$ ) and above the $\sigma_{0}=27$ or $27.15 \mathrm{~kg} \mathrm{~m}^{-3}$ isopycnal in the observations and ECCO state estimate respectively. We calculated the wind stress source of vorticity and the flux of vorticity out of the region from observations as well as in the ECCO v4 adjoint state estimate and placed the terms in context by diagnosing the wind stress forcing over the eastern portion of the basin.

The advective vorticity flux term is robustly negative and the wind stress forcing over the western region is zero or negative (all mean terms quoted in Table 2-1). The vorticity flux is on the same order as the wind stress forcing over the eastern region, consistent with a large-scale balance between wind stress forcing in the interior of the gyre and friction, or another source of positive vorticity, in the western region. Further insight can be gained from the calculation of advective vorticity flux alone, independently of the size of the other terms. The vorticity flux is an order of magnitude smaller than its cumulative absolute value, indicating that there is a significant inertial component to the gyre circulation. These 

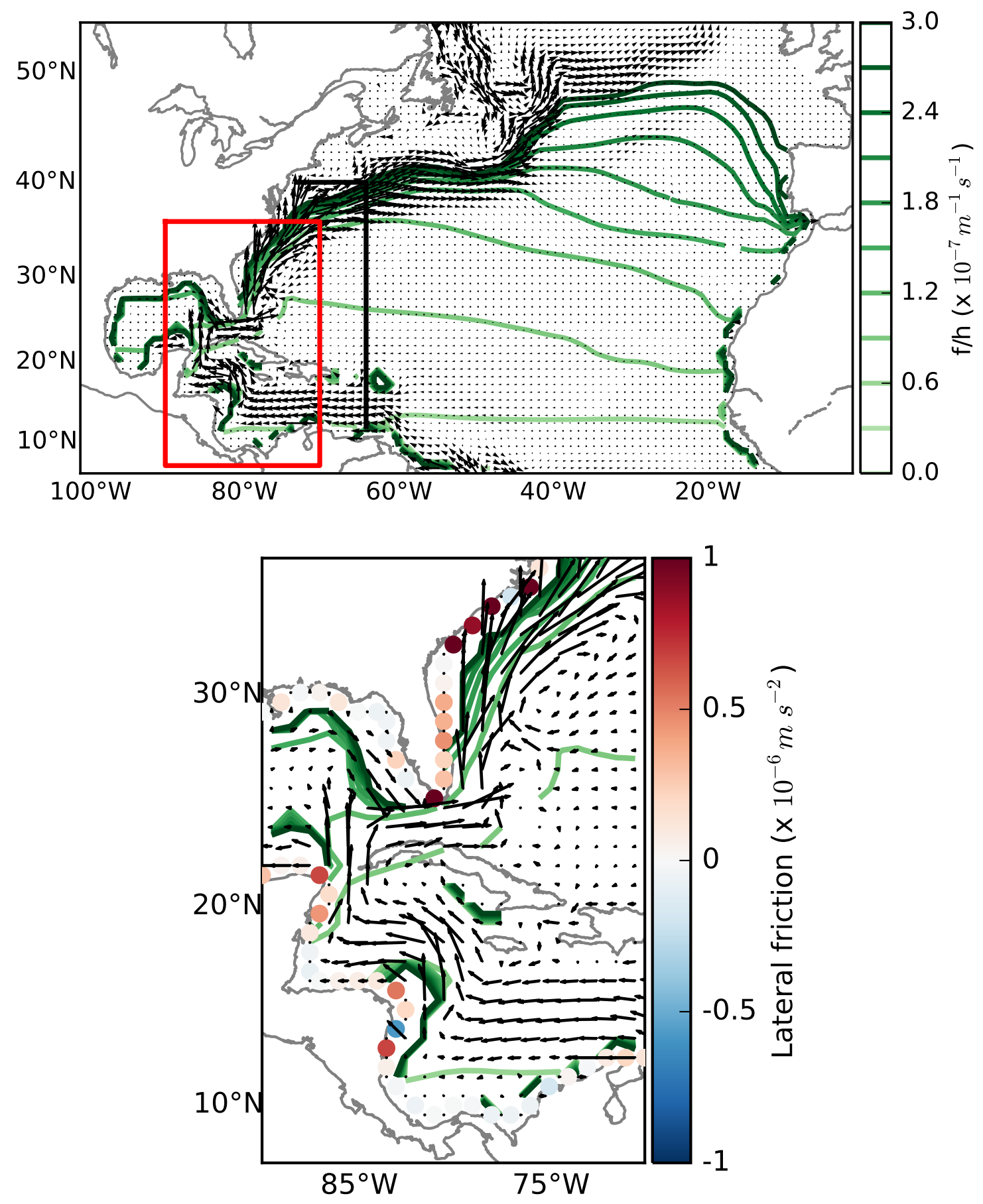

Figure 2-9: Maps showing the relationship of $\mathrm{f} / \mathrm{h}$ contours, time mean depth-averaged flow and lateral friction for the study volume in the ECCO model. Time mean $\mathrm{f} / \mathrm{h}$ contours are shown in green, as described in the legend, where $\mathrm{h}$ is the depth of the $\sigma_{0}=27.15 \mathrm{~kg} \mathrm{~m}^{-3}$ isopycnal in the ECCO model. Time mean velocity depth-averaged between the $\sigma_{0}=27.15 \mathrm{~kg} \mathrm{~m}^{-3}$ isopycnal and the ocean surface are shown in black arrows. Thick black line in the upper panel denotes the separation between the western and eastern regions in the ECCO model analysis. The red box in the upper panel denotes the area shown in the lower panel. The lower panel includes time mean lateral friction values along the integration contour, as described in the legend, with dark red values corresponding to large positive inputs which enable flow to higher $\mathrm{f} / \mathrm{h}$. 
results hold across our calculations from observations and the ECCO state estimate.

In the ECCO model, we can diagnose the size of the remaining terms and find a balance between the negative advective vorticity flux, and positive contributions from lateral friction, bottom drag and wind stress forcing over the western region, as summarized in Figure 2-8 and Table 2-2. Note that the wind stress forcing over the western region has the greatest variability of all terms, and its variability is not included in the sum of terms in Figure 2-8. The large variability in the wind stress forcing term is the reason that there is large uncertainty in the sum of all budget terms, $0 \pm 0.4 \mathrm{~m}^{2} \mathrm{~s}^{-2}$. The positive sign of the time mean wind stress forcing term over the west is likely unique to the ECCO model. The largest positive term in the ECCO budget is the lateral friction term, which is a parameterization of smaller scale processes, such as internal wave breaking and eddy decay that are likely significant in the ocean.

Because we diagnose the time series of each of these terms in the ECCO model, we can split the full time mean term into its component due to the time mean and eddy fields. For example, this decomposition for the advective vorticity flux would be

$$
<-\int(f+\widetilde{\zeta}) \overline{\mathbf{u}} \cdot \mathbf{d} \mathbf{n}>=-\int(f+<\widetilde{\zeta}>)<\overline{\mathbf{u}}>\cdot \mathbf{d} \mathbf{n}-\int<\widetilde{\zeta}^{\prime} \overline{\mathbf{u}}^{\prime}>\cdot \mathbf{d} \mathbf{n},
$$

where $\langle\cdot\rangle$ denotes temporal averages and primes are deviations from the time mean; correlations between these deviations lead to an eddy term. In the ECCO model we find that each term is dominated by its component due to the time mean fields, as shown in Table 2-2, but this is likely not the case in the ocean, which has more eddy variability than is present in the ECCO model.

Another way to think about our findings is in a Lagrangian framework. The robustly negative advective vorticity flux implies a source of positive vorticity in the volume, so that a parcel that enters the domain will acquire vorticity in the volume, through frictional or eddy processes, for example, and leave the domain with higher vorticity than it entered with, hence the net outward flux of vorticity.

Our results are consistent with the literature, in which both Stommel-Munk and inertial theories are thought to be relevant to gyre circulation, with inertial theories applying primarily to recirculation gyres (e.g. Cessi, 1990; Waterman and Jayne, 2011). Vallis (2006), for example, shows analytically that friction will always be necessary to balance wind stress forcing on the large scale, even in a nonlinear system. Fox-Kemper and Pedlosky (2004) further gave a specific instance in which friction and inertia can play leading order roles in the dynamical balance of a gyre, with increased friction in a small viscous sublayer.

Our budget of the vorticity of the depth-averaged flow is fundamentally different than the Hughes and de Cuevas (2001) budget of barotropic vorticity that highlights the role of topography, and claims that western boundary currents are inviscid. In the limit of homogeneous density, budgets of barotropic vorticity reduce to budgets of vorticity whereas 
budgets of the vorticity of depth-averaged vorticity reduce to budgets of PV. Barotropic vorticity budgets highlight how the layer thickness and vorticity components of PV adjust in order to conserve PV. An inertial barotropic vorticity balance indicates that PV is conserved to first order, but does not address the forcing and dissipation that alters PV.

Our budget framework can be thought of as highlighting the processes which allow the crossing of $\mathrm{f} / \mathrm{h}$ contours. As is evident in Figure 2-9, the time and depth-averaged velocity field follows $\mathrm{f} / \mathrm{h}$ contours to first order. Upon closer inspection, however, it is clear that the Gulf Stream crosses to higher $\mathrm{f} / \mathrm{h}$ contours from the point that it enters the Caribbean as it extends northward along the continental slope. This crossing of $f / h$ contours is facilitated in large part by lateral friction from the Florida Straits to Cape Hatteras, as highlighted in Figure 2-9. Figure 2-9 also shows that there is no clear Gulf Stream separation in the ECCO depth-averaged flow field, which is another critical difference in character between the "real" and ECCO Gulf Streams.

The relative consistency of the budget term sizes is notable given the limitations of our datasets. There are only two realizations of the A22 hydrographic line suitable for this analysis, and we can only calculate the relative vorticity associated with along-section variations in velocity. There is significant variability in the advective vorticity flux term in the ECCO model and it is questionable to what degree a synoptic section can represent the long term mean. Eddy contributions to the vorticity budget have similarly not been accounted for in the observations. There is also significant variability in the wind stress forcing term, especially over the western region (see Figure 2-5.) The wind stress forcing calculated from different wind stress products show a range of possible mean values. Though we showed using the ECCO state estimate that variations in layer thickness do not have a significant impact on the wind stress forcing term, the uncertainties in the Gouretski and Koltermann (2004) climatology are also present in our calculation of wind stress forcing. The ECCO state estimate gives an idea of the temporal variability of the budget, but it has significant limitations. Its one degree resolution means that its Gulf Stream is quite different than the observed Gulf Stream, for example, and that eddies are not resolved.

This study focuses on terms in a budget of the vorticity of the depth-averaged flow on the gyre scale. Though this framework simplifies to a budget of shallow-water PV in the limit of a layer of homogeneous density, we do not claim to accurately depict all transformations of PV that occur in the ocean. However, we can draw an interesting parallel to the recent work of Deremble et al. (2014) on PV budgets in the North Atlantic. Their focus is on Ertel PV flux and they discuss the circulation of PV in thin isopycnal layers. Their figure 5 shows how this circulation changes with the depth of the isopycnal: PV is advected in the anticyclonic subtropical gyre direction on the $\sigma_{0}=26 \mathrm{~kg} \mathrm{~m}^{-3}$ isopycnal, the strength of the advection weakens with depth and is in the reverse direction on the $\sigma_{0}=27 \mathrm{~kg} \mathrm{~m}^{-3}$ isopycnal because of thermobaric effects. In our study we have integrated from the $\sigma_{0}=27 \mathrm{~kg} \mathrm{~m}^{-3}$ isopycnal to 
the surface, so that the fluxes that we quote represent an average over the vertical face of the whole layer. Deremble et al. (2014) calculate the net westward flux of PV of about $-4 \mathrm{~m}^{2} \mathrm{~s}^{-2}$ in the $\sigma_{0}=26 \pm 0.25 \mathrm{~kg} \mathrm{~m}^{-3}$ isopycnal layer ${ }^{1}$, which is similar to the average vorticity fluxes out of the layer that we calculate $\left(-2.4 \pm 2.5 \mathrm{~m}^{2} \mathrm{~s}^{-2},-5.3 \pm 2.2 \mathrm{~m}^{2} \mathrm{~s}^{-2}\right.$, and $-3.0 \pm$ $0.1 \mathrm{~m}^{2} \mathrm{~s}^{-2}$ from the A22 2003, 2012 and ECCO products respectively). In their framework, this layer is representative of the interior of the subtropical gyre because it is deep enough to include the Gulf Stream throughout the year and shallow enough that it is not affected by thermobaricity. Our values are representative of the subtropical gyre circulation as a whole because we average over the entire layer. It is interesting that their representative value is on the same order as the one we calculated both from A22 observations and the ECCO model despite the differences in our approaches.

Our integrated budget of the vorticity of depth-averaged flow indicates that a positive source of vorticity is necessary in the western subtropical North Atlantic, and is consistent with the Stommel and Munk models of subtropical gyres in which wind stress forcing in the interior is balanced by friction in the western boundary. At the same time, we show through analysis of the form of the vorticity flux that there is a significant inertial component to the circulation. In the ECCO model, we found that the positive source of vorticity is composed principally of lateral friction, wind stress forcing and bottom drag. Precisely how this budget closes in the real ocean remains an open and interesting question.

\footnotetext{
${ }^{1}$ This value is the difference in flux between points 4 and 12 on their figure 6 , which are located slightly west of $60^{\circ} \mathrm{W}$.
} 
Chapter 3

\section{Water Mass Properties in the Deep Western BOUNDARY CURRENT}




\subsection{Abstract}

Observations of the DWBC at Line $\mathrm{W}$, on the continental slope about $39^{\circ} \mathrm{N}$, from 1995 to 2014 reveal water mass changes that are consistent with changes upstream in the Labrador Sea. This is most evident in the deep Labrador Sea Water (dLSW) water mass associated with intense winter convection in the Labrador Sea in the early 1990s. The intense deep convection period resulted in a cold, fresh, and thick anomaly in the dLSW range. The arrival of this cold-fresh-thick anomaly is evident 3-7 years later in shipboard measurements at Line W. Further, the transition from cold-fresh to warm-salty properties in the dLSW range and thinning of the dLSW layer is measured by the Line W moored array from 20042014 with statistical confidence. Additional datasets along the path of the DWBC provide further evidence for advection of the cold-fresh anomaly and indicate that stirring between the boundary and the interior increases south of the Flemish Cap. The consistency of the data with realistic advective and mixing time scales is assessed using the Waugh and Hall (2005) model framework. The data are found to be consistent with a mean transit time of approximately 5 years, with a leading order role for both advection and mixing. 


\subsection{Introduction}

The Deep Western Boundary Current (DWBC) carries cold, dense water from the highlatitude North Atlantic to the South Atlantic along the western boundary of the North Atlantic basin. The first indication of the DWBC appeared in water property measurements from the Meteor Expedition (1925-1927) in the Atlantic led by Alfred Merz and Georg Wüst (Wüst, 1935).

Stommel et al. (1958) proposed a dynamical explanation for the DWBC, which can be framed in terms of Potential Vorticity (PV), a dynamical tracer that is conserved barring forcing and dissipation. They hypothesized that vertical stretching associated with new deep water production led to poleward flow throughout the basin towards sources of deep water. The PV and mass balance of the system could only be achieved by an equatorward boundary current focused on the western edge of the basin. Several years later the DWBC was confirmed by Swallow and Worthington (1961) near Cape Hatteras using acoustically tracked floats and hydrography.

Since this time there have been many direct current measurements using moored instrumentation that confirm this equatorward flow pattern; in the subpolar gyre at $53^{\circ} \mathrm{N}$ (Dengler et al., 2006; Fischer et al., 2010, 2015) at $47^{\circ} \mathrm{N}$ (Rhein et al., 2011; Mertens et al., 2014) and at the Grand Banks (Schott et al., 2004, 2006), close to the inter-gyre boundary at Line $\mathrm{W}^{1}$ about $39^{\circ} N$ (see Figure 3-1) (Joyce et al., 2005; Toole et al., 2011; Peña-Molino et al., 2012), at Cape Hatteras (Pickart et al., 1990), at 26.5 N (Meinen et al., 2013a; Srokosz and Bryden, 2015), at $16^{\circ} \mathrm{N}$ (Kanzow et al., 2006, 2008; Köhler et al., 2014) and in the Southern Hemisphere at 8 and $11^{\circ} \mathrm{S}$ (Dengler et al., 2004; Hummels et al., 2015) and at $34^{\circ} \mathrm{S}$ (Meinen et al., 2013b; Dong et al., 2014).

As North Atlantic deep water spreads equatorward in the DWBC, it subducts and is shielded from atmospheric forcing. Consequently, deep water can be tracked throughout the world ocean using its PV signature, its characteristic temperature and salinity properties and anthropogenic tracer concentrations, such as Chloroflourocarbons (CFCs), which are imprinted on it by the atmosphere at its source. Many studies have taken advantage of these unique tracer properties to track the equatorward spreading of North Atlantic deep water along the western boundary of the North Atlantic (Lynn and Reid, 1968; Talley and McCartney, 1982; Pickart et al., 1989; McCartney, 1992; Doney and Jenkins, 1994; Molinari et al., 1998; Smethie, 1993; Smethie et al., 2000; Smethie and Fine, 2001; Stramma et al., 2004; LeBel et al., 2008; Kieke et al., 2009; Rhein et al., 2002, 2015; Van Sebille et al., 2011). CFC studies of the DWBC, such as Pickart et al. (1989) found along-boundary tracer spreading rates that were several times slower than mean speeds in the DWBC $(O)$

\footnotetext{
${ }^{1}$ Line $\mathrm{W}$ is named for L. Valentine Worthington, for his many significant contributions to measuring and understanding North Atlantic current systems.
} 


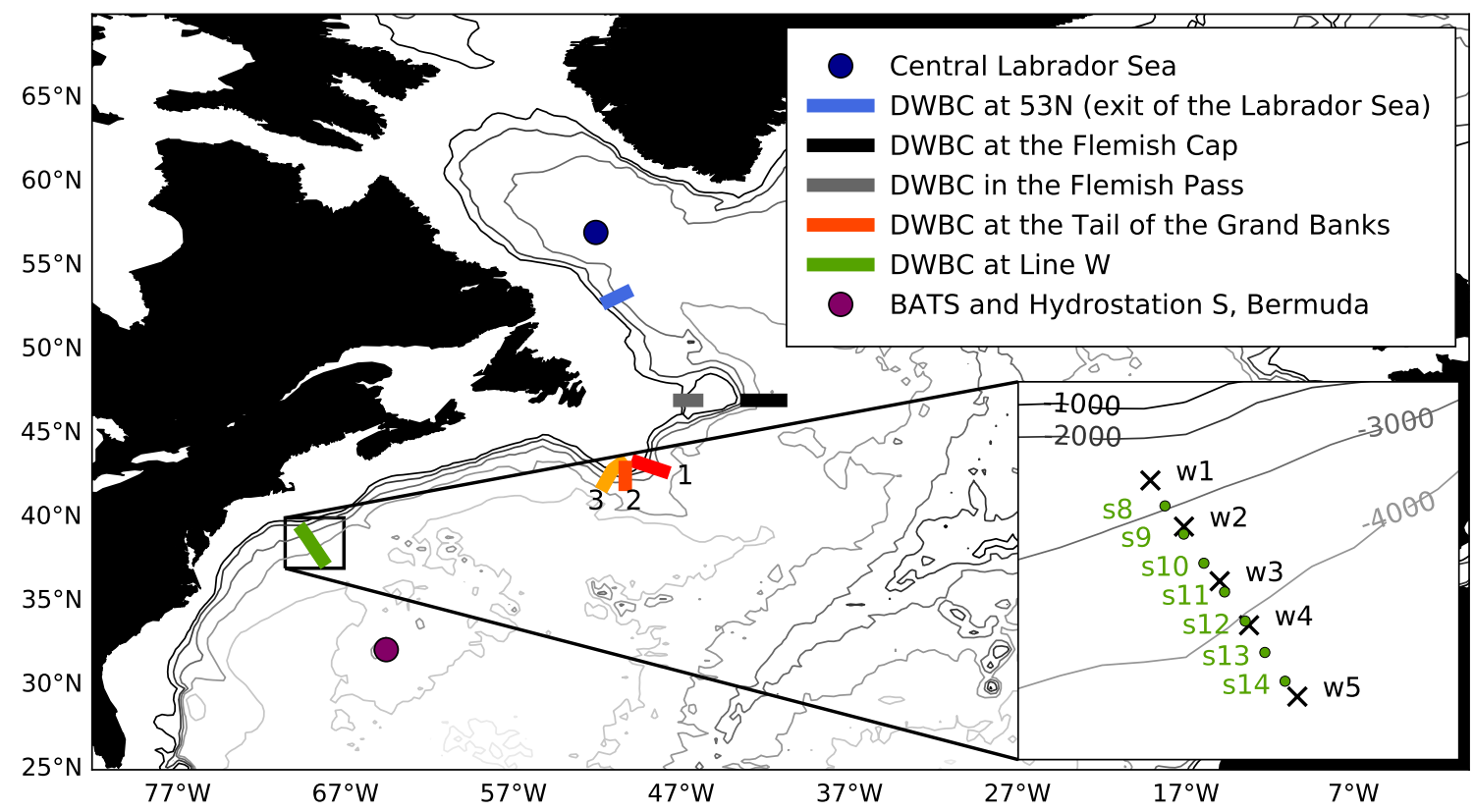

Figure 3-1: Map of dataset locations along the path of the Deep Western Boundary Current as described in the legend. Inset in the lower-right shows Line W mooring positions (in black) and station positions used in this study (in green). Bathymetric contours are drawn every $1000 \mathrm{~m}$ and labeled in the inset.

$\left.2 \mathrm{~cm} \mathrm{~s}^{-1}\right)$ and $O\left(5-10 \mathrm{~cm} \mathrm{~s}^{-1}\right)$ respectively); indicating stirring with the interior as well as recirculation. This discrepancy between effective spreading rates and mean DWBC speeds was confirmed by Bower and Hunt (2000) using acoustically tracked floats.

This study reports on water mass property changes in the DWBC on the continental slope of New England (between 35 and $40^{\circ} \mathrm{N}$ ), where Line W moorings were operational from April 2001 to May 2014 (see Figure 3-1). The moored array was complemented by hydrographic surveys that date back to 1994 and include measurements of anthropogenic tracers, such as CFCs and ${ }^{129}$ I (Smith et al., 2016). The primary sources of observed variability at the Line W moorings are associated with topographic Rossby waves (Thompson and Luyten, 1976), warm core rings shed from Gulf Stream meanders (Peña-Molino et al., 2010) and deep cyclones (Andres et al., 2015), all of which have associated time scales on the order of months. In this work, we focus on water mass property changes on annual to decadal time scales. The DWBC at Line $\mathrm{W}$ has little vertical shear, typical velocities of 5 to $10 \mathrm{~cm} \mathrm{~s}$ and a mean deep water transport of $25 \mathrm{~Sv}$, with a range of comparable size (Toole et al., 2011). Upon averaging the mooring data, several velocity cores emerge, seemingly aligned with different water masses of the DWBC (Toole et al., 2011).

The DWBC is composed of water masses formed at high-latitude that are commonly differentiated by their origin. The intermediate water masses in the $\mathrm{DWBC}$ are formed in the Labrador Sea (found from about 500-2000 $\mathrm{m}$ at Line W). Labrador Sea Water is 
often split into upper Labrador Sea Water (uLSW) which may be formed in the boundary current of the Labrador Sea, and deep Labrador Sea Water (dLSW) which is formed in the center of the Labrador Sea (Pickart et al., 1997; Lazier, 2001; Stramma et al., 2004; Kieke et al., 2006; Rhein, 2000; Rhein et al., 2007). The strength of the convection and corresponding water mass signature in the central Labrador Sea can vary dramatically from year to year, so that the distinction between upper and deep LSW is not always welldefined or instructive (Yashayaev, 2007). In the early 1990s, for example, there was intense deep convection in the central Labrador Sea, during a period of very high North Atlantic Oscillation (NAO) index (Hurrell, 1995; Marshall et al., 2001b). The NAO is a strong pattern of decadal climate variability that is associated with changes in temperature, windiness, and precipitation over the North Atlantic (Hurrell and Deser, 2010). The NAO index is the difference of atmospheric pressure at sea level between the Icelandic low and the Azores high, and quantifies the severity of this pattern. When the NAO index is high, storms are focused in the subpolar North Atlantic, leading to an increase in deep convection.

During the high NAO period in the early 1990s, Labrador Sea convection reached $2000 \mathrm{~m}$ and the water mass formed was extremely cold and fresh. As apparent in Figure 3-2, the surface of the central Labrador Sea is fresh due to ice melt and river runoff (Yashayaev, 2007). When there is deep convection, this fresh water is mixed into the water column, forming a fresh anomaly at depth. In the second half of the 1990s, the NAO index decreased, convection reached shallower depths and the Labrador Sea became warmer and saltier throughout the water column.

The dLSW layer we define is associated with the extreme deep convection of the early 1990s, which is in keeping with other studies, such as Stramma et al. (2004). Our water mass definitions are broadly consistent with other definitions in the literature, and we compare them in Table 3-1. Note that although we describe water mass properties, in Section 3.5 we analyze properties in small uniform bins of neutral density, independent of water mass definitions. This analysis forms the basis of our choice of the uLSW/dLSW boundary.

The densest water mass classes of North Atlantic origin are formed in the Nordic Seas. These waters flow over sills into the subpolar North Atlantic and are often referred to as Overflow Waters (OWs) (Swift, 1984; Hansen and Osterhus, 2000; Dickson et al., 2002). OWs are separated into lighter Iceland-Scotland Overflow Waters (ISOW) and denser Denmark Strait Overflow Waters (DSOW), named for their origins. Iceland-Scotland Overflow Waters entrain saline Atlantic waters as they descend into the deep Icelandic basin and the resulting North East Atlantic Deep Waters (NEADWs) only consist of about 1/3 ISOW (Van Aken and De Boer, 1995; Yashayaev et al., 2007). NEADWs in the DWBC are also referred to as Gibbs Fracture Zone Water (GFZW), since they primarily cross the Mid-Atlantic Ridge through the Charlie Gibbs Fracture Zone (Saunders, 1994; Schott et al., 1999; Stramma et al., 2004) and ISOW (Toole et al., 2011). We only analyze the NEADW component of 

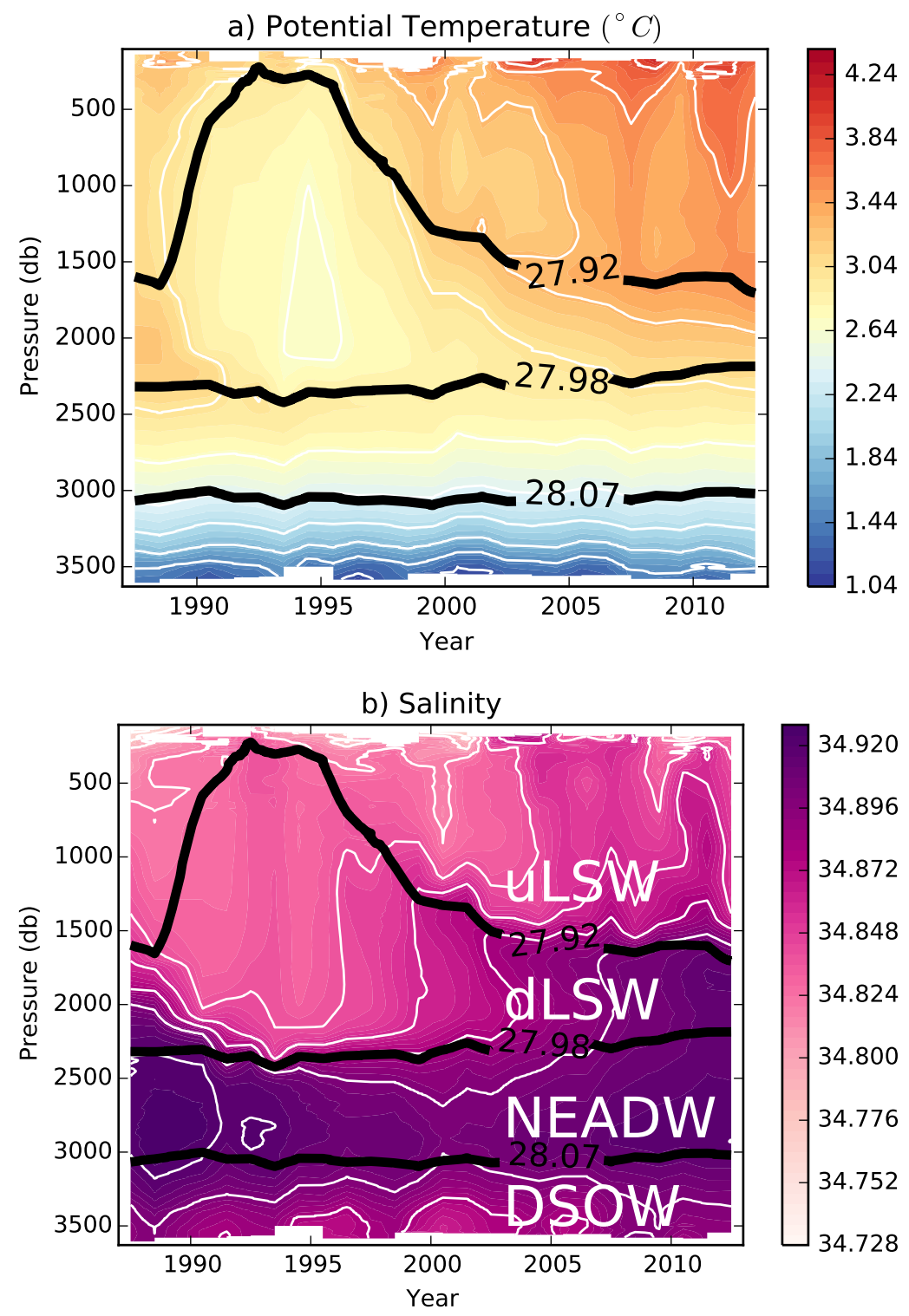

Figure 3-2: Time evolution of a) potential temperature and b) salinity profiles in the central Labrador Sea. Thick labeled lines highlight the boundaries of the Labrador Sea water mass we focus on. Water mass labels in b) apply to both panels. The layer is thickest, freshest and coldest in the early/mid 1990s and becomes thinner, saltier and warmer throughout the 2000s.

OW in this work, because it was measured at Line W every year, unlike DSOW, which was not measured by the early hydrographic surveys that did not sample sufficiently far offshore. Additionally, DSOW has been the focus of recent studies using the Line W CFC and ${ }^{129} \mathrm{I}$ tracer data (Andres et al., 2015; Smith et al., 2016). We use standard NEADW water mass definitions, derived from tracer and water mass properties, and these are also reported in Table 3-1.

Recent work has investigated the relative importance of the DWBC and interior pathways 


\begin{tabular}{|c|c|c|c|}
\hline & This work & $\begin{array}{c}\text { Toole et al. 2010 } \\
\text { Pena-Molino et al. 2011 }\end{array}$ & $\begin{array}{c}\text { Yashayaev et al. 2007 } \\
\text { van Sebille et al. 2011 }\end{array}$ \\
\hline $\mathrm{uLSW}$ & $\gamma_{n}=27.80(160 / 690)$ & & $\sigma_{2}=36.5(105 / 630)$ \\
$\mathrm{dLSW}$ & $\gamma_{n}=27.92(1180 / 1320)$ & $\gamma_{n}=27.897(890 / 1100)$ & $\sigma_{2}=36.82(300 / 960)$ \\
$\mathrm{NEADW}$ & $\gamma_{n}=27.98(2300 / 1940)$ & & $\sigma_{2}=36.97(2470 / 2150)$ \\
& $\gamma_{n}=28.07(3050 / 2790)$ & & $\sigma_{2}=36.98(2550 / 2240)$ \\
\hline
\end{tabular}

Table 3-1: Water mass definitions used in different studies in $\mathrm{kg} \mathrm{m}^{-3}: \gamma_{n}$ is neutral density and $\sigma_{2}$ is potential density referenced to $2000 \mathrm{~m}$. Values in parentheses are mean depths of the isopycnal in central Labrador Sea and at Line $\mathrm{W}$ in $m$. Central Labrador Sea mean depths calculated from 1988-2013 and Line W mean depths calculated from w3 data (Nov 2001- May 2014).

for the equatorward spreading of deep water, building on the findings of DWBC tracer studies that indicated a role for stirring and recirculation in setting DWBC transit times. In a set of experiments from 2003 to 2005 , less than $10 \%$ of acoustically tracked isobaric floats seeded in the DWBC in the LSW layer at $50^{\circ} \mathrm{N}$ rounded the Tail of the Grand Banks in the DWBC, pointing to the importance of exchange between the boundary current and the interior as well as interior pathways (Bower et al., 2009, 2011, 2013). Float deviations into the interior at the Grand Banks as well as the subsequent interior pathways were then found to be replicated in high-resolution models (Gary et al., 2011, 2012; Lozier et al., 2013). Recirculations at the exit of the Labrador Sea, which may be relevant to the ejection of floats from the DWBC (Xu et al., 2015), have been observed by Hogg (1983) and Lavender et al. (2000), and there is growing appreciation for the importance of this so-called "transition zone" near the Flemish Cap (Rossby, 1999; Pérez-Brunius et al., 2004; Rössler et al., 2015; Buckley and Marshall, 2015).

To assess the degree of connectivity in the DWBC, we compare salinity, potential temperature and layer thickness changes in the DWBC at Line W, which is downstream of this high-energy region, to changes upstream of it in the Labrador Sea. In Section 3.3 we introduce the Line W and central Labrador Sea datasets. This is followed, in Section 3.4, by an analysis of Line $\mathrm{W}$ mooring data in $\theta-S$ space which illustrates a shift in water mass properties consistent with changes in the Labrador Sea. We then turn to the shipboard data, in Section 3.5, and quantify the coherence between Labrador Sea and Line W hydrographic anomalies in neutral density space. In Section 3.6, we average within water mass layers and draw on additional observations of the DWBC (see Figure 3-1) to further investigate the evolution of the water mass properties along the path of the DWBC. Finally, in Section 3.7 we use the analytic solution to an advection-diffusion model to estimate the transit time 
distributions from the Labrador Sea to Line W. We discuss our results in Section 3.8.

\subsection{Datasets}

\subsubsection{Line $\mathrm{W}$}

The Line W moored array consisted of five moorings (w1-w5) deployed on the continental slope Southeast of Cape Cod from April 2004 to May 2014 spanning isobaths between 2200 and 4000m, with the central mooring (w3) in the water since November 2001 (see Figure 3-1). A sixth mooring was added to the array in May 2008, but does not enter our analysis as its water mass properties and variability are dominated by the Gulf Stream, whose mean position is between moorings w5 and w6 (Peña-Molino et al., 2010; Andres et al., 2015). Peña-Molino et al. analyzed water mass property changes at w3 from 2001-2008 and found significant changes in the potential vorticity, temperature and salinity, consistent with changes in water mass production at subpolar latitudes. Here, we extend this analysis, including all moorings that sample the DWBC (w1-w5) and data from 2008-2014, and compare directly with other datasets.

Between the two settings (2004-2008 and 2008-2014) of the Line W array, the distribution of moored instruments changed, complicating the construction of consistent time series. From 2004-2008, w1, w3 and w5 were equipped with McLane Moored Profilers (MMPs), profiling sensors which measure velocity, temperature and conductivity, and moorings w2 and w4 were equipped with fixed instruments: vector averaging current meters (VACMs) and Sea Bird Electronics temperature/conductivity sensors $(\mathrm{T} / \mathrm{S})$. Further details about the instrument setup from 2004-2008 can be found in Toole et al. (2011). In the 2008-2014 setting, this distribution was reversed: w2 and w4 were equipped with MMPs and w1, w3, w5 and w6 were equipped with fixed instruments.

The MMPs typically sampled in bursts of 4 one-way profiles every 5 days. Averaging the individual profiles in these bursts acts to filter out ageostrophic high-frequency signals because they were separated by 9.5 hours, which is approximately half the local inertial and three-quarters of the semi-diurnal tidal period (Silverthorne and Toole, 2009); the resulting dataset has 5 day temporal resolution and $2 \mathrm{db}$ vertical resolution. The fixed instruments have much higher temporal resolution, 15 minutes for $\mathrm{T} / \mathrm{S}$ and 30 minutes for VACM data, but their vertical resolution varies, and is on the order of $100 \mathrm{db}$.

To address the difference in temporal sampling, the fixed instrument data were low pass filtered and subsampled to 5 day intervals, which is more than sufficient for the purposes of this study. Because of the irregular positions of fixed instruments (for the purposes of measuring DWBC transport) the subsampling in depth was more involved. For each mooring, the MMP data were subsampled at the mean positions of the fixed instruments that preceded or followed them. The subsampled data were then interpolated vertically using a 
cubic spline and the mean properties (temperature, salinity, layer thickness, velocity) within water mass boundaries described in the introduction from the full and subsampled datasets were compared. Since the magnitude and variability of these water mass properties were well-reproduced by the subsampled data, the subsampled MMP dataset was used throughout to avoid measurement bias. The same cubic spline vertical interpolation was applied to the fixed sensor data.

Shipboard hydrographic measurements at fixed station positions which extend most of the way to Bermuda (see Figure 3-1) were occupied once or twice a year from 1994-1997 and 2001-2014. More details on the shipboard data may be found in Andres et al. (2015). The hydrographic parameters used in this work represent a mean in neutral density space weighted by layer thickness of all stations that lie between the locations of moorings w1 and w5 to facilitate comparison of moooring and hydrographic data.

All shipboard and mooring data are available through national data archives as well as at the Line W website, http://www.whoi.edu/science/PO/linew/.

\subsubsection{Central Labrador Sea}

Central Labrador Sea data sets were provided by Igor Yashayaev. What follows is his description of the construction of annual mean vertical profiles shown in Figure 3-2.

The locations of observations from ship surveys and Argo floats selected for our analysis, shown in Figure 3-3, represent the epicenter of deep convection in the Labrador Sea. From the west and southwest, the boundary is the $3250 \mathrm{~m}$ isobath on the Labrador slope. Each annual vertical profile was constructed by averaging temperature, salinity and pressure of all available vertically interpolated observations in $\sigma_{2}$ bins with $\Delta \sigma_{2}=0.005 \mathrm{~kg} \mathrm{~m}^{-3}$. If any $\sigma_{2}$ bin did not have sufficient data points to form reliable estimates, its size was expanded incrementally until it included the needed number of observations or it reached $0.020 \mathrm{~kg} \mathrm{~m}^{-3}$. Further details of the calculations of robust seawater property statistics involving two types of statistical weights applied to the values in each $\sigma_{2}$ bin may be found in the "Methods and definitions A: Construction of time series" section of Yashayaev (2007). The estimates found in the $\sigma_{2}$ bins were then vertically interpolated to every 5 dbar, forming a uniform time-pressure array. 


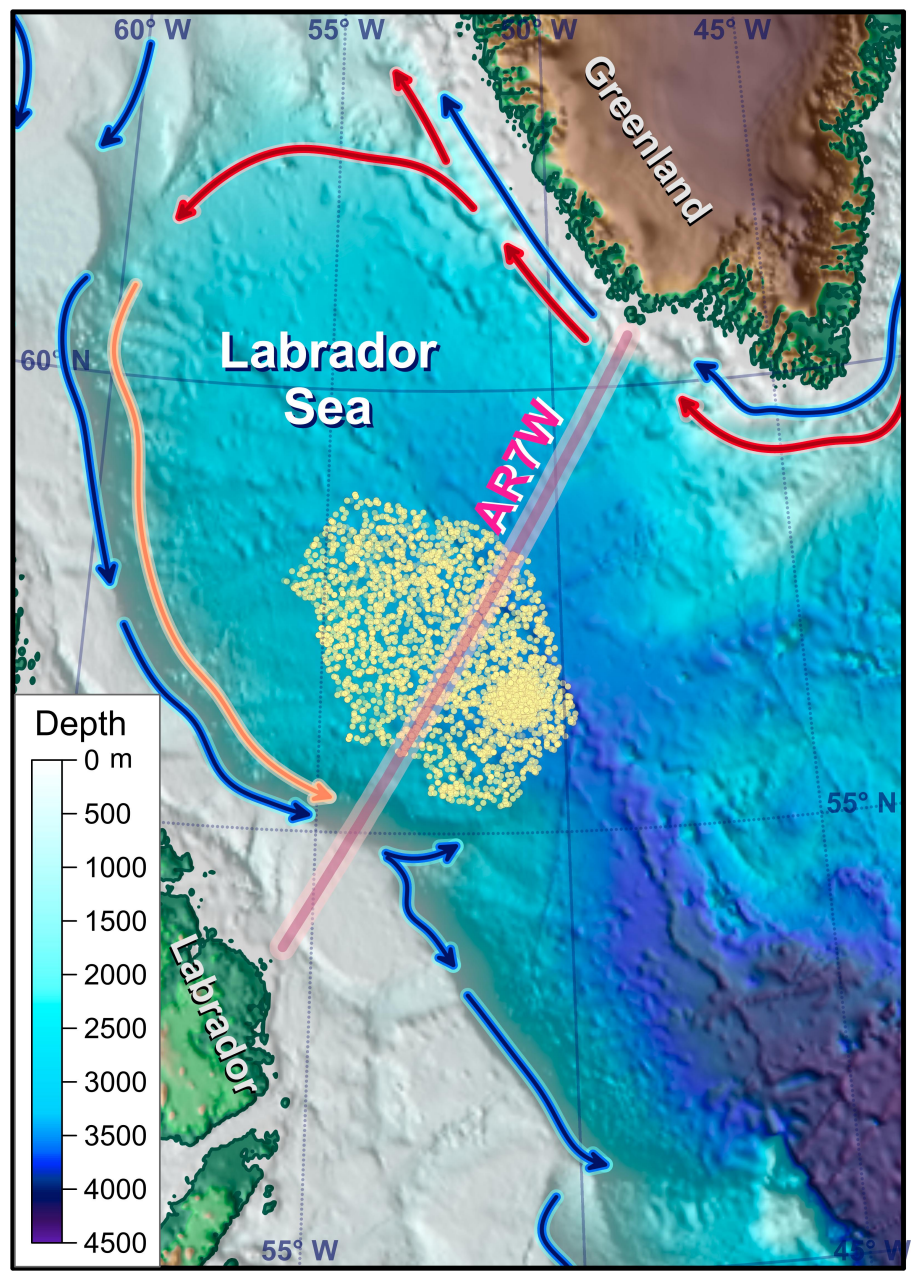

Figure 3-3: Map of the Labrador Sea highlighting observation locations used to form the annual average central Labrador Sea profiles (yellow points). Both shipboard and Argo profiles are included in this dataset. The AR7W repeat hydrography section is highlighted in pink and the arrows depict the mean circulation in the region schematically. Figure from Igor Yashayaev.

\subsection{Line $\mathbf{W}$ mooring $\theta-S$ shifts}

The Line $\mathrm{W}$ mooring $\theta-S$ properties lie between the central Labrador Sea $\theta-S$ properties and properties measured monthly at the Bermuda Atlantic Time Series (BATS), which is $75 \mathrm{~km}$ southeast of Bermuda, as shown in Figure 3-4. 


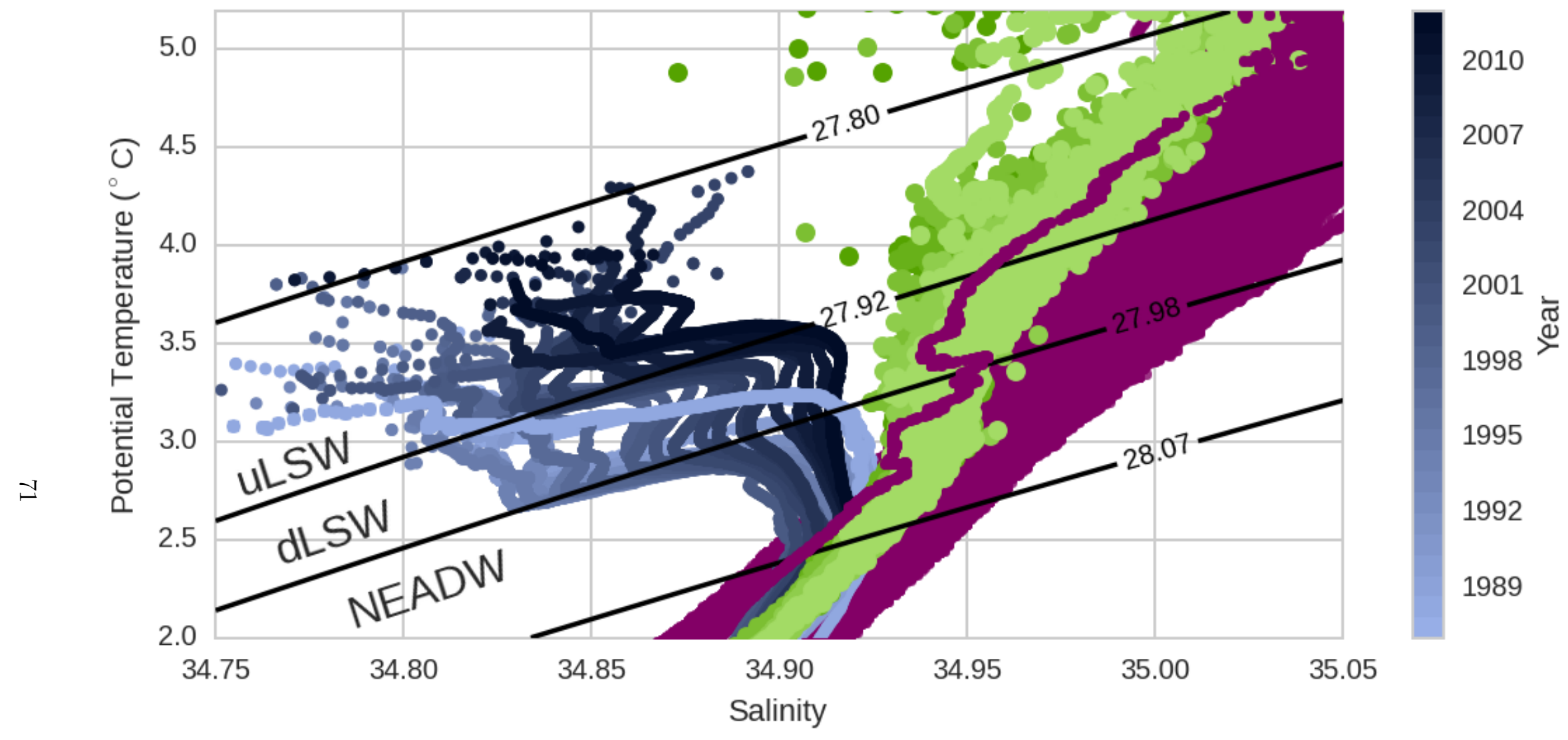

\section{Central Labrador Sea}

Figure 3-4: $\theta-S$ properties in the central Labrador Sea (blue), at Line W moorings (green) and BATS (magenta). Shades of green correspond to measurements at different Line W mooring sites as in Figure 3-5. Central Labrador Sea profiles are color-coded by year as in the colorbar. Line W mooring profiles include data from 2001 to 2014. BATS profiles include data from 1989 to 2015, measured monthly. The magenta line on top of the green Line W profiles is an example of a cold-fresh profile with apparent LSW influence at BATS. Black lines are neutral density boundaries (referenced to $1000 \mathrm{db}$ ) between water masses for reference. 
The waters formed in the Labrador Sea are the coldest and freshest in the LSW density range in the North Atlantic basin, so that deviations in the cold-fresh direction in the LSW range can be assumed to emanate from the Labrador Sea (Talley and McCartney, 1982). The LSW contribution to the $\theta-S$ properties can be seen in the cold-fresh bump visible in the shape of both the Line $\mathrm{W}$ and BATS datasets around $\theta=3.5, S=34.93$. $\theta-S$ properties at BATS are generally warmer and saltier than at Line $W$, because of the influence of Mediterranean Water (Talley and McCartney, 1982; Van Sebille et al., 2011) and the more circuitous path that LSW takes to get to BATS. However, the overlain cold-fresh profile observed at BATS shown in Figure 3-4 demonstrates that LSW influences $\theta-S$ properties there as well, as shown in Curry et al. (1998) and Phillips and Joyce (2007). Cold-fresh properties are frequently measured at BATS, as will be shown in Section 3.6. The property space between the cold-fresh profile depicted in Figure 3-4 and the remaining visible magenta BATS measurements is also filled with BATS measurements, but they are hidden by Line W measurements in Figure 3-4 to highlight the $\theta-S$ structure at Line W.

The dramatic changes in $\theta-S$ properties in the Labrador Sea from 1988 to 2014 are also illustrated in Figure 3-4. The extreme convection in the early 1990s resulted in a cold-fresh peak in the dLSW range. Thereafter, throughout the late 1990s and 2000s the water in the dLSW range became warmer and saltier. In their Figure 6, Peña-Molino et al. (2010) show that the annual mean $\theta-S$ properties at Line W's central mooring, w3, similarly change from cold-fresh to warm-salty within their dLSW layer from 2001-2008; reflecting the changes upstream in the Labrador Sea several years prior.

The general pattern of warming and salinifying in the dLSW range that continued at Line $\mathrm{W}$ to the end of the program is apparent at moorings w1-w5. In addition to this continuing change in annual mean properties from cold-fresh to warm-salty, we also show that there is a change in the range of $\theta-S$ properties present at Line $\mathrm{W}$ in the span of a year. To do this analysis, we use the subsampled mooring properties (see section 3.3.1).

As shown in Figure 3-5, in 2005 the $\theta-S$ properties measured over the year cover the full range of properties measured throughout the Line $\mathrm{W}$ record, from cold-fresh to warm-salty. This range progressively decreased and the annual mean became warmer and saltier in the LSW range. By 2013, the $\theta-S$ properties present were limited to the warm-salty edge of all sampled $\theta-S$ space. Because of our data subsampling and the fact that both MMP and fixed instrument measurements are present in both settings of the moored array, measurement bias has been minimized in this result. 

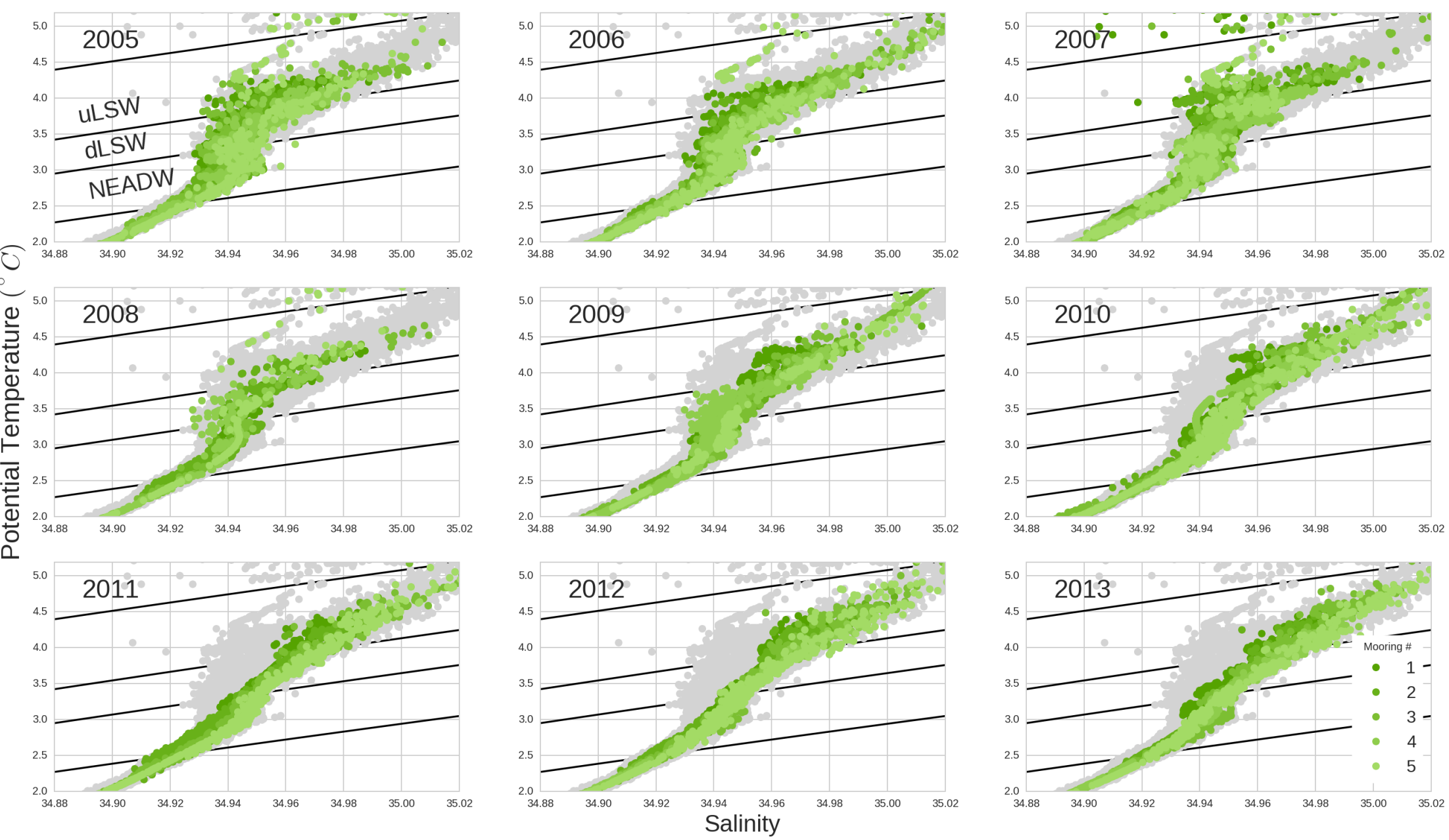

Figure 3-5: Evolution of $\theta-S$ properties at Line W moored array from 2005 to 2013. Grey background points are all profiles measured with 5 day resolution at Line $\mathrm{W}$ moorings w1-w5 and overlain green points are profiles measured in the year in the top left of the panel. Moorings are differentiated by shades of green as described in the legend. There is a progression from a broad set of profiles spanning the measured $\theta-S$ space in 2005 to a focused concentration on the warm-salty edge of the measured $\theta-S$ space in 2013 . There are fewer data in 2008 because of measurement gaps. Black lines are neutral density boundaries (referenced to 1000db) between water masses for reference. 
This transition from a broad set of $\theta-S$ properties at the beginning of the moored observations to a narrow set of $\theta-S$ properties on the warm-salty edge of $\theta-S$ space is due to the decreasing presence of the water formed during intense deep convection in the central Labrador Sea in the early 1990s. The narrow set of $\theta-S$ properties at the end of the record can be interpreted as the basic state of $\theta-S$ properties at Line W. When there is water present that was formed by intense deep convection in the Labrador Sea, anomalously coldfresh $\theta-S$ properties are observed. As discussed in more detail in section 3.7, the shift is from a large range of properties to a narrow range (instead of a monotonic shift in properties from cold-fresh to warm-salty) because there is a broad distribution of transit times from the Labrador Sea as well as stirring with the interior. These mixing processes cause the Line $\mathrm{W}$ properties to occupy less of a range in $\theta-S$ space than the Labrador Sea $\theta-S$ properties. Stirring with interior waters, which are generally warmer and saltier than the DWBC, accounts for the fact that the properties at Line $\mathrm{W}$ are warmer and saltier on the whole than the Labrador Sea $\theta-S$ properties.

\subsection{Coherence between hydrographic anomalies in neutral den- sity space}

Although a water mass property shift is observed at the Line $\mathrm{W}$ moorings that is consistent with changes in the Labrador Sea, the moored observations did not start early enough to record the first arrival of waters formed during the extreme deep convection event of the early 1990s. In order to quantify the coherence between the Labrador Sea and Line W datasets, we turn to the shipboard measurements, since there are at least yearly shipboard observations available in the late 1990s (1994-1997) as well as during the moored array period (2001-2014.) Notice that there is a three year gap between these two periods.

To gauge the coherence of the two datasets, we first convert to neutral density coordinates (Jackett and McDougall, 1997) so that the vertical coordinate for the Labrador Sea and Line $\mathrm{W}$ data are consistent and to remove the effects of heaving isopycnals. We calculate salinity and layer thickness within $0.01 \mathrm{~kg} \mathrm{~m}^{-3}$ neutral density bins and then subtract the record mean of the time series within each bin. The data shown in Figure 3-6 and used in the subsequent analysis have been linearly interpolated in time. We do not show potential temperature because it is compensated for by salinity changes within neutral density bins.

We expect coherence between these two datasets that are connected by the DWBC because salinity on neutral density surfaces acts as a passive tracer in the absence of mixing processes. Layer thickness between neutral density surfaces can also be treated as a tracer as it is proportional to the inverse of integrated planetary potential vorticity $\left(P P V=\frac{f}{\rho} \frac{\partial \sigma}{\partial z}\right)$, which is conserved in the absence of forcing and mixing. However, layer thickness is limited by the height of the water column and gradients in layer thickness are associated with 
geostrophic baroclinic flow.

Our choice of water mass layers is based on the coherence of the Labrador Sea and Line W datasets. In Figure 3-6, it is apparent that Line $\mathrm{W}$ properties and their variability are similar within water mass layers at Line $\mathrm{W}$, but that they are distinct from the layers above and beneath them, as one might expect. In the Labrador Sea, properties are relatively similar throughout the water column, particularly when dLSW is being formed and its properties are set by ventilation.

The largest signal in the Labrador Sea is associated with the intense deep convection in the early 1990s, as can be seen in Figure 3-6. During those years, the NAO was in an extreme positive phase (Hurrell, 1995), and the Labrador Sea was cold and stormy, so that fresh surface properties were mixed into the interior through convection. As the conditions for intense deep convection persisted, the fresh anomaly penetrated to larger densities, until about 1994. The persistent convection is also highlighted by the layer thickness anomalies in Figure 3-6; large positive layer thickness anomalies are seen at ever denser densities throughout the early 1990s. All bins within this dLSW layer became saltier and thinner through the 2000s. The dLSW layer in the Labrador Sea has a distinct maximum in mean bin layer thickness and its salinity is homogeneous and fresher than the underlying NEADW. In the NEADW layer in the Labrador Sea, a weaker decadal cycle is apparent. As is welldocumented (Yashayaev et al., 2007), there is a salinity maximum in the NEADW layer and, as it is not convectively formed, there is no signature in the mean bin layer thickness profile associated with this layer.

Throughout most of this record, the surface in the central Labrador Sea is denser than the uppermost bin in the uLSW layer, as shown by the grey shading in Figure 3-6. This is most apparent during the intense deep convection of the early 1990s, when the surface water is almost as dense as the upper boundary of dLSW. In the 2000s, other convection events are visible in the uLSW layer. Most notably, in the year 2000, convection reached $27.92 \mathrm{~kg} \mathrm{~m}^{-3}$, or $1000 \mathrm{~m}$ (Yashayaev, 2007). The unexpected return of deep convection in 2008 (Vage et al., 2008; Yashayaev and Loder, 2009) is also perceptible as a dense and fresh anomaly, though its signature is very weak compared to the intense convection of the early 1990s. In the uLSW layer there is a general thickening throughout the 2000s, though the anomalies are not as large as the deep convection event in the dLSW layer. There is a peak in mean bin layer thickness in the uLSW layer in the Labrador Sea, though it is smaller than the peak in the dLSW layer. The uLSW layer is the freshest part of the water column in the Labrador Sea. 

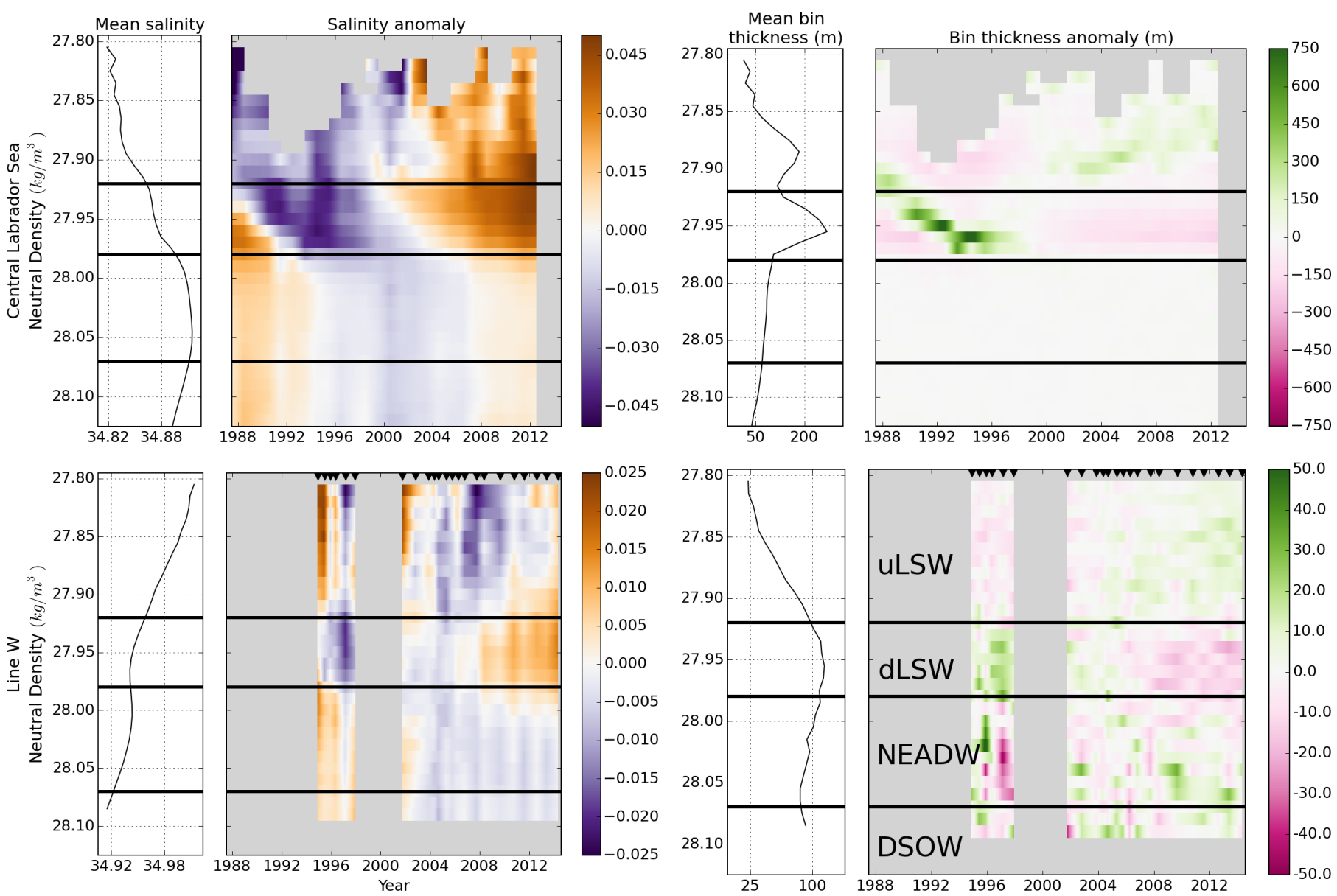

Figure 3-6: Water mass property anomaly time series and mean profiles in the central Labrador Sea (top row) and at Line W (bottom row) in neutral density space. Left panels show salinity anomalies within $0.01 \mathrm{~kg} \mathrm{~m}^{-3}$ neutral density bins and the corresponding mean profile on the left; the right panels show the same for the thickness of the neutral density bins. Horizontal black lines are neutral density boundaries between water masses, as labeled in the Line $\mathrm{W}$ thickness anomaly plot (bottom right). Black triangles at the top of Line $\mathrm{W}$ anomaly plots indicate when measurements were made. 
At Line W, both salinity and layer thickness anomalies are smaller than in the Labrador Sea: the salinity anomaly colorbars in Figure 3-6 differ by a factor of two and the layer thickness colorbars by an order of magnitude. Strictly, the two anomaly time series cannot be compared directly because the respective means are taken over different periods of time, and the Line $\mathrm{W}$ time series includes a 3 year gap in the middle. Despite this, some general similarities in pattern can be observed. In particular, there is a striking fresh anomaly in the dLSW layer at Line W in the late 1990s. Throughout the 2000s, the dLSW at Line W became saltier. There is also thinning within the dLSW layer at Line W throughout the record concurrent with the general pattern from fresh to salty. As in the Labrador Sea, there is a peak in mean bin layer thickness in the dLSW layer as well as a salinity minimum in the mean profile.

The NEADW layer freshens throughout the Line W record. The size of NEADW anomalies are of similar order as in the central Labrador Sea. Since NEADW does not have a convective origin, there is no PV or layer thickness tag associated with it, so that layer thickness changes at Line $\mathrm{W}$ are not coherent throughout the layer.

In the uLSW layer at Line W, the anomaly signals are much noisier and appear uncorrelated with anomalies in the Labrador Sea; this is at least partially because the uLSW layer is not always present in the central Labrador Sea. The largest anomaly in the record is a surface intensified fresh anomaly from 2005-2008 that may be associated with convection in the uLSW in the early 2000s. The layer thickness anomalies in the uLSW become larger throughout the Line W record, just as they do in the central Labrador Sea. Peña-Molino et al. (2010) find similar patterns in their analysis of PPV at Line W mooring w3. They find a migration of the PPV minimum at w3 from the dLSW layer to the uLSW layer over the course of the first setting of the moored record (2001-2008); this is the equivalent of a thinning dLSW layer and thickening uLSW layer.

To quantify the coherence between the anomaly time series shown in Figure 3-6, we took the lagged correlation between the datasets within each $0.01 \mathrm{~kg} \mathrm{~m}^{-3}$ neutral density bin, linearly interpolating across the data gap in Line W.

The Labrador Sea and Line W salinity and temperature anomalies are lag correlated with a coefficient greater than 0.75 for neutral densities below 27.92, as shown in Figure 3-7a. This encompasses the dLSW layer, as well as the NEADW layer. Layer thickness anomalies are not as well correlated in general, with no correlation in the NEADW layer; likely because there is no layer thickness signature in this layer in the Labrador Sea. The layer thickness anomalies do have correlations higher than 0.75 for the top of the dLSW layer, though. Note that none of these correlations are statistically significant since the anomaly time series are short compared to the event time scale and consequently have few degrees of freedom.

The lag which maximizes the correlation has a distinct structure in depth, as seen in 
Figure 3-7b, with smaller lags in the dLSW layer than in the underlying NEADW layer. The envelope around the correlation-maximizing lag shows the range of lags which give more than 0.95 times this maximum correlation, which indicates how sharp the peak in correlation is as a function of lag. The lag which maximizes the correlation between the Labrador Sea and Line $\mathrm{W}$ in the dLSW layer is 2-6 years. We only show the correlation maximizing lag for correlations that are higher than 0.75 . The lag calculated from layer thickness corresponds well with the lag calculated from salinity and temperature anomalies in the upper part of the dLSW layer, with smaller uncertainty.

The final element of this analysis, shown in Figure 3-7c, is the ratio of the magnitudes of lagged anomaly time series, again shown only where the correlation is higher than 0.75 . The standard deviations of the $\theta$ and salinity time series in the middle of the dLSW layer at Line $\mathrm{W}$ are 0.2 and 0.25 times their original size in the Labrador Sea respectively. The ratio of layer thicknesses in the dLSW layer ranges from 0.05 to 0.25 ; the ratio is smallest for the neutral density bin that has the highest mean thickness. This ratio increases to about 0.4 for $\theta$ and 0.5 for salinity in the NEADW layer. The large decrease in anomaly amplitude in the dLSW is to be expected, due to recirculation as well as stirring with other water masses on the way from the Labrador Sea to Line W. Because the extreme convection in the Labrador Sea was a unique input of a thick, cold and fresh layer into these intermediate densities, and because the lag we found is consistent with other studies, as we will discuss in Section 3.8, we can be sure that this signal is emanating from the Labrador Sea. The fact that the anomalies in the NEADW in the Labrador Sea are much smaller than in the dLSW layer, means that we can be less certain that the signal in this density class emanates from the Labrador Sea.

In summary, salinity and layer thickness anomalies in neutral density space show similar patterns in the central Labrador Sea and at Line W. A lagged correlation analysis between the Labrador Sea and Line W shows that dLSW salinity, temperature and layer thickness anomalies are well correlated with a lag of 2-6 years, though salinity and temperature anomalies are 4-5 times larger in the Labrador Sea. Potential temperature and salinity anomalies in the NEADW layer are only about 2 times smaller at Line $W$ than in the Labrador Sea, indicating less stirring of NEADW. NEADW salinity and temperature anomalies are correlated at lags of 5-10 years, but because the anomaly amplitudes are smaller, we have less confidence in these lag times. In the next section (Section 3.6), we include other datasets and describe the evolution of water mass averaged properties along the western boundary, leading towards quantification of transit time distributions that are consistent with the evolution of dLSW and NEADW properties in Section 3.7. 
$0.000 .05 \quad 0.100 .15 \quad 0.200 .250 .300 .35$
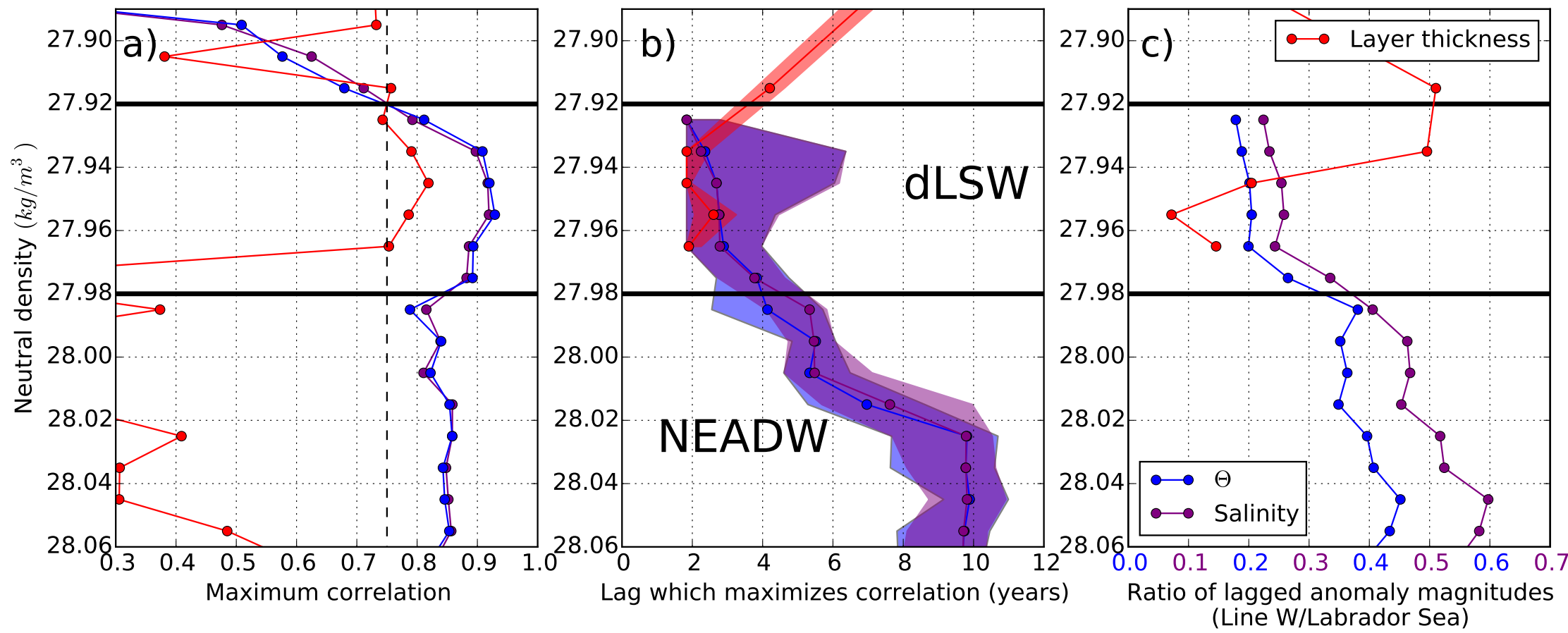

Figure 3-7: Results from lagged correlation analysis between Labrador Sea and Line W layer thickness, potential temperature and salinity anomalies within neutral density bins (shown in Figure 3-6). The legend in c) applies to all panels. a) Maximum correlation between Labrador Sea and Line W anomaly time series within the neutral density bins shown on the y axis. Vertical dashed line is at 0.75 ; only bins with correlations higher than 0.75 are shown in the next panels. b) The lag which yields the maximum correlation (shown in a). The envelope around the correlation-maximizing lag shows the range of lags which give more than 0.95 times this maximum correlation for each property. c) Ratio of the standard deviations of Labrador Sea and Line W lagged anomaly time series. Top x-axis corresponds to layer thickness ratios and bottom axis is for potential temperature and salinity ratios, which are two times larger. Horizontal black lines are neutral density boundaries between water masses, as labeled in $\mathbf{b}$ ). 


\subsection{Water mass class averages}

Having identified water mass classes by quantifying the coherence between water properties in the Labrador Sea and Line W in neutral density space, we now average within them, compare absolute quantities and include more measurement locations (see Figure 3-1) to assess the along-path evolution of water mass properties.

The additional measurement locations that we analyze include a time series at the exit of the Labrador Sea at $53^{\circ} \mathrm{N}^{2}$, where yearly shipboard hydrographic measurements have complemented a moored array across the DWBC since 1996 (Dengler et al., 2006; Fischer et al., 2010, 2015). Our focus is on the shipboard data because they include salinity measurements. We include all profiles between 52.9 and $53.4^{\circ} \mathrm{N}$, and west of $50^{\circ} \mathrm{W}$, which maximizes the consistency between sections and captures the DWBC. The second dataset that we analyze in the subpolar gyre is further downstream at the Flemish Cap and Flemish Pass, at $47^{\circ} \mathrm{N}$ 3 (Rhein et al., 2011; Mertens et al., 2014; Schneider et al., 2015). We define the DWBC at the Flemish Cap as lying west of $43^{\circ} \mathrm{W}$, which includes only the DWBC on the continental slope as defined in Mertens et al. (2014) and the Flemish Pass as everything in the shallow pass west of $46^{\circ} \mathrm{W}$, between the "nose" of the Grand Banks and the Flemish Cap. We also analyze 3 hydrographic sections off the Tail of the Grand Banks ${ }^{4}$, in each case considering data out to the $3500 \mathrm{~m}$ isobath. Finally, we analyze data at Bermuda Atlantic Time Series (BATS), $75 \mathrm{~km}$ southeast of Bermuda $\left(31^{\circ} 40^{\prime} \mathrm{N}, 64^{\circ} 10^{\prime} \mathrm{W}\right)$, and Hydrostation S, $25 \mathrm{~km}$ southeast of Bermuda $\left(32^{\circ} 10^{\prime} \mathrm{N}, 64^{\circ} 30^{\prime} \mathrm{W}\right)$ (Phillips and Joyce, 2007). Note that there are monthly measurements available at BATS and bi-weekly at Hydrostation S, but in Figure 3-8 we highlight annual mean values to compare directly with the other datasets.

\section{Water mass salinity}

The salinity in the uLSW layer (see Table 3-1 for water mass definitions) in the subpolar gyre is generally increasing throughout our record, as shown in Figure 3-8a. The salinities in the central Labrador Sea are only shown where the lighter uLSW boundary exists, which is only a few points near the beginning and end of the record. At $53^{\circ} \mathrm{N}$, the full uLSW layer is sampled throughout the record. This is consistent with the conclusion in Pickart et al. (1997) that uLSW is always present in the boundary current, and is often formed there. The salinities at the exit of the Labrador Sea, at the Flemish Cap and Flemish Pass are well-matched. At the Tail of the Grand Banks, though the patterns are similar, the uLSW is generally saltier, likely because of interaction with the North Atlantic Current, and the associated high eddy kinetic energy in the region (Rossby, 1996). The Line W shipboard

\footnotetext{
${ }^{2} 53^{\circ} \mathrm{N}$ data were provided by J.Fischer, M.Visbeck, J.Karstensen and P.Handmann, GEOMAR, Kiel

${ }^{3} 47^{\circ} \mathrm{N}$ data were provided by M.Rhein, C.Mertens, D.Kieke and L.Schneider, MARUM, Bremen University

${ }^{4}$ Tail of the Grand Banks data were extracted from the CCHDO database (cchdo.ucsd.edu)
} 
uLSW salinity time series looks similar to the Flemish Pass data, consistent with the idea that the shallow Flemish Pass is a shortcut for uLSW export to the subtropical gyre, as shown by Bower et al. (2009) and Schneider et al. (2015). However, the Line W mooring data, shown in Figure 3-9, illustrate a lack of coherence between mooring uLSW salinity across the array. The uLSW salinity at BATS and Hydrostation S, which agree very well with each other, as shown in Figure 3-8g, reveal no clear pattern and have a larger range because of the presence of Mediterranean Overflow Water.

In the dLSW layer, the primary signal in the Labrador Sea is the freshening in the early 1990s, with the freshest years in 1994 and 1995 (see Figure 3-8b). Through the 2000s, the dLSW becomes saltier, and this trend is apparent at $53^{\circ} \mathrm{N}$ and at the Flemish Cap as well. Similar patterns are observed at the Tail of the Grand Banks (see Figure 3-1 for track locations) and Line W several years later. At Line W, there is a freshening trend from 1994 to 1997 and a generally saltier period in the 2000s. There is a statistically significant trend in the dLSW recorded by the Line W moored array from 2004 to 2014 (w3 from 2001), as shown in Figure 3-9. This trend is found in moorings w1-w5 and is consistent with an increase of $0.012 \pm 0.002 /$ decade; the corresponding trend in temperature is $+0.014 \pm 0.02^{\circ} \mathrm{C} /$ year . Quoted uncertainties are 95\% confidence intervals based on a decorrelation time scale of 3 months, which gives about 100 degrees of freedom. All trends and uncertainties are listed in Table 3-2. Note that, unlike in Peña-Molino et al. (2010), warm core rings have not been removed from this record, and trends are significant despite their influence.

At BATS and Hydrostation S, the dLSW salinity spans much more of a range than at Line $\mathrm{W}$, but does show some indication of a minimum annual mean salinity at the end of 2003. This could be because of the slower and less consistent arrival of the cold-fresh dLSW formed in the central Labrador Sea in the early 1990s, but we cannot show this conclusively here. The influence of LSW in shaping the water mass properties at Hydrostation $\mathrm{S}$ has been shown in Curry et al. (1998), Joyce et al. (2000) and Phillips and Joyce (2007). The fact that the dLSW salinity minimum is earlier at Line $\mathrm{W}$, which is about the same distance from the Labrador Sea as Bermuda is, indicates that the DWBC is a faster, more coherent, equatorward path for dLSW than any interior pathway to Bermuda. 

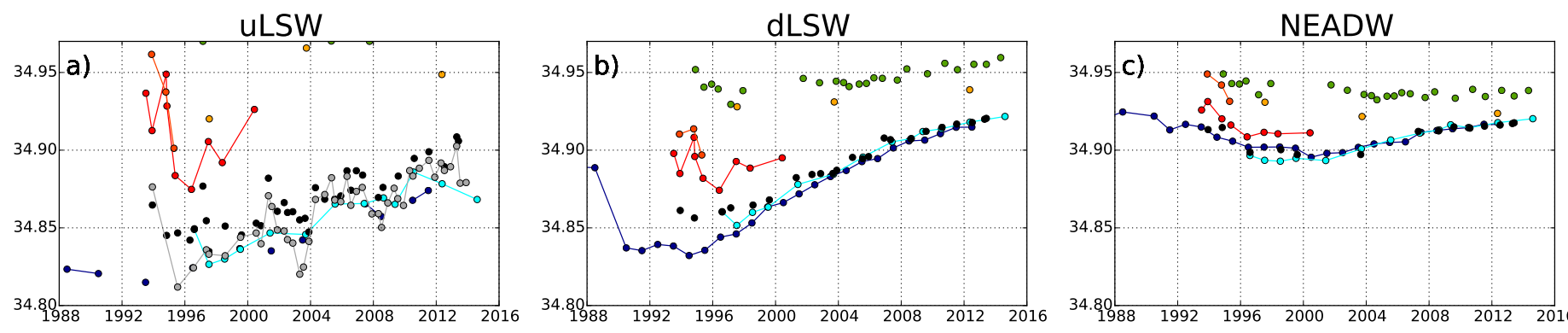

- Central Labrador Sea

- DWBC at $53 \mathrm{~N}$

- DWBC at the Flemish Cap

- DWBC at the Flemish Pass

- Tail of the Grand Banks (track 1)

- Tail of the Grand Banks (track 2)

- Tail of the Grand Banks (track 3)
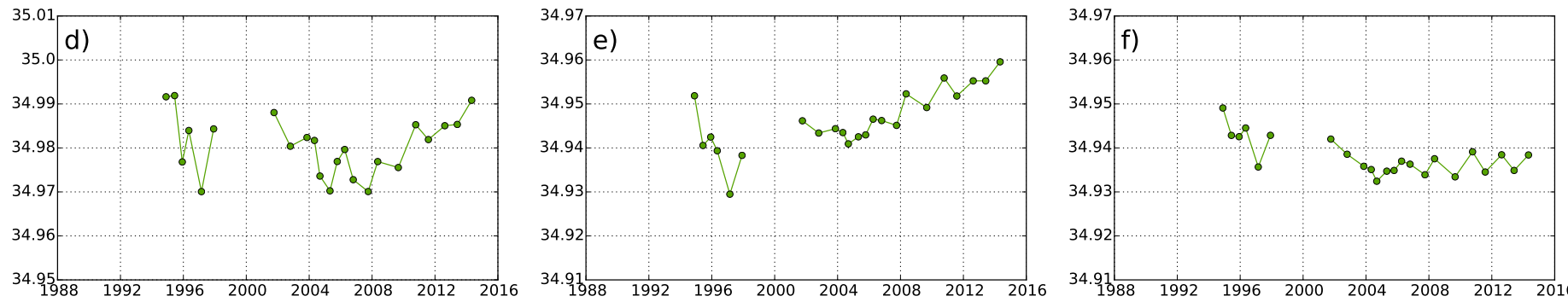

DWWC at Line W
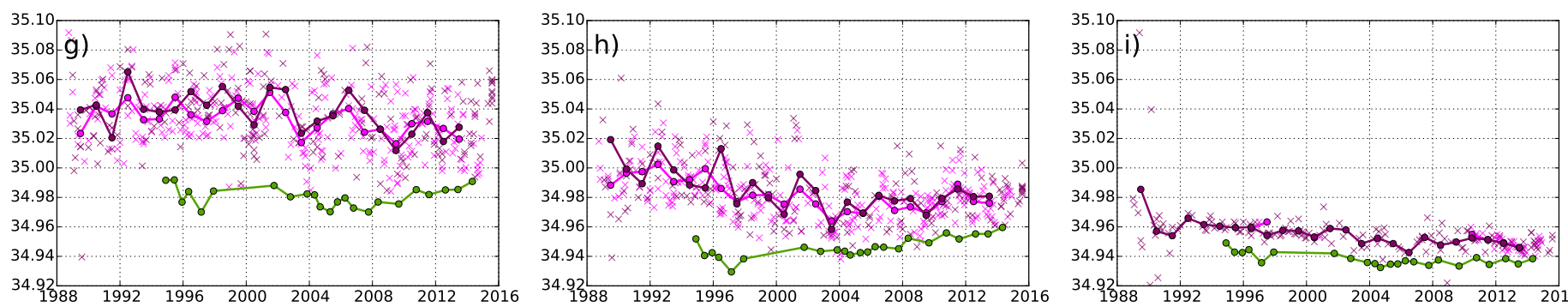

- Hydrostation S, Bermuda

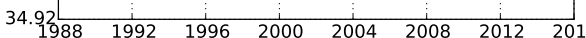

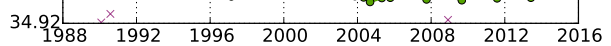

\section{$\longrightarrow$ BATS, Bermuda}

Figure 3-8: Mean salinity within water masses for datasets along the path of the DWBC. Panels are split into water masses: uLSW (left, a d g), dLSW (center, $\mathbf{b}$ e f) and NEADW (right, $\mathbf{c} \mathbf{f} \mathbf{i}$ ) and location: subpolar gyre (top, a b c), Line W (center, $\mathbf{d}$ e f), Hydrostation S and BATS (bottom, g h i). Subpolar gyre datasets are differentiated in the legend. Each row shows the same range of salinities to facilitate side-by-side comparison, though this range is shifted in the case of Line W uLSW (d) which is saltier than the underlying water masses. uLSW salinity is shown only when the full uLSW density range is present. Where it fits in the range chosen for each location, the Line W data is included. The salinity time series in the dLSW in the subpolar gyre and at Line $\mathrm{W}$ have similar shapes with a $\approx 5$ year lag, reflecting the propagation of the fresh anomaly associated with intense deep convection in the Labrador Sea of the early 1990s to the subtropical gyre. 


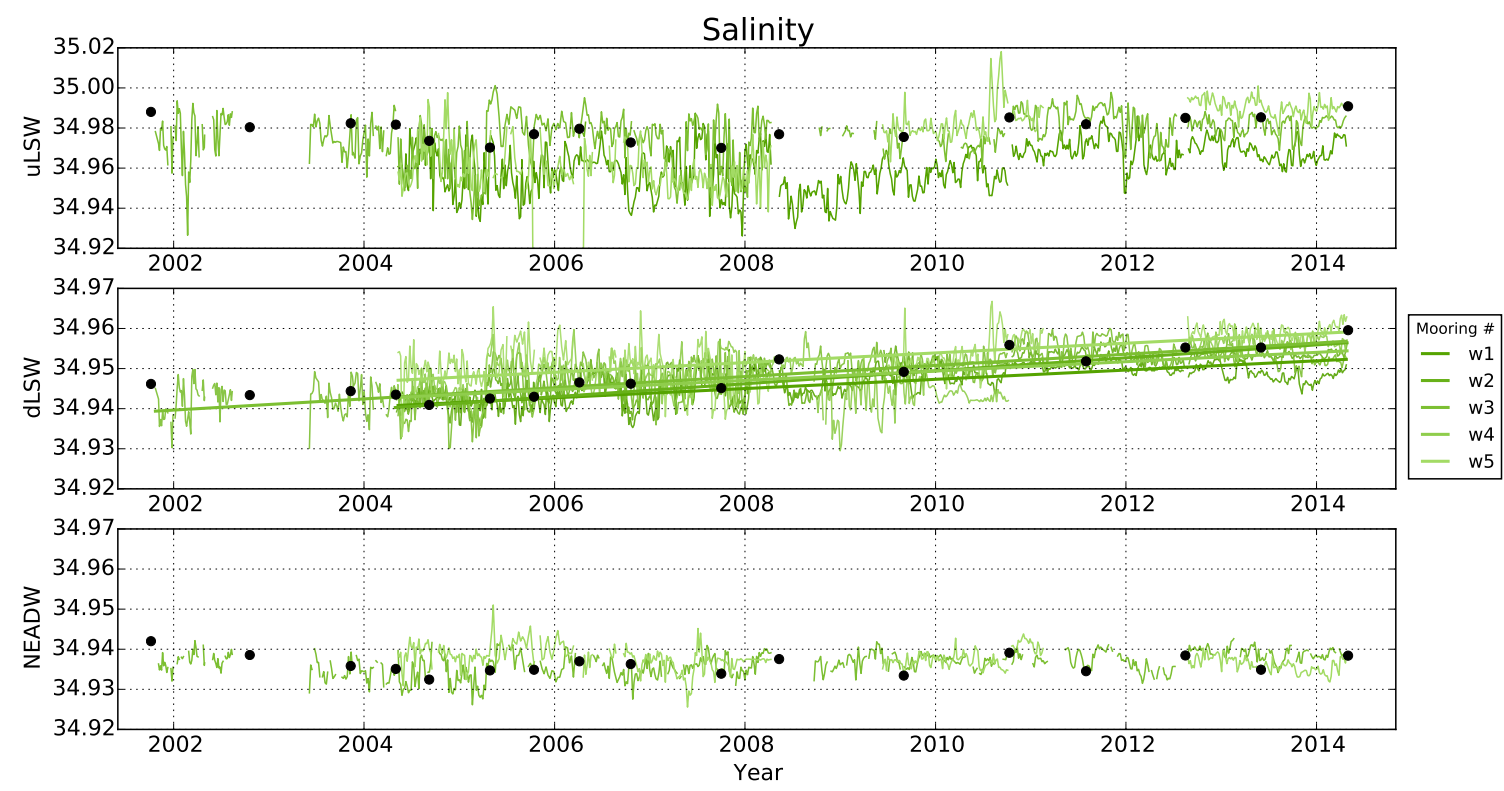

Figure 3-9: Mean salinity within water masses measured by Line W moorings. uLSW (top row) was measured by all but mooring w4, dLSW (middle row) by all 5 moorings and NEADW (bottom row) by moorings w3 and w5. The legend shows which shade of green corresponds to which mooring measurement. Linear fits to dLSW salinity are overlain, highlighting the statistically significant increase in salinity from 2002 to 2014 at all Line W moorings. The slope values and uncertainties are quoted in Table 3-2. Black dots are shipboard data for comparison. Shipboard salinity averages are weighted by layer thickness; the better correspondence with the thicker w1 than the thinner w 5 uLSW is expected.

Salinity changes in the subpolar NEADW layers are about three times smaller than those in either LSW layers, as shown in Figure 3-8. From the Labrador Sea to the Flemish Cap, the salinities are very similar, with a minimum about the year 2000. As in the other layers, the salinity is higher at the Tail of the Grand Banks and highest at Line W. The moored record, in Figure 3-9, shows that there is little variability in the NEADW layer compared to the shallower LSW layers. At BATS, there is a slight freshening in the NEADW layer throughout the record; Hydrostation S only measured deep enough to sample NEADW in the late 2000s. As explained in Section 3.5, and will be explored in detail in Section 3.7, because the variability in the salinity of NEADW in the Labrador Sea is small to begin with, the connectivity of this layer assessed from this analysis is more uncertain.

\section{Water mass layer thickness}

The evolution of water mass layer thickness along the path of the DWBC is less straightforward to interpret than water mass salinity. As explained in Section 3.5, salinity primarily acts as a passive tracer on neutral density surfaces, while layer thickness is constrained by the height of the water column.

The thickness of the uLSW layer decreases along the path of the DWBC, with the 
largest values in the Labrador Sea, followed by the exit of the Labrador Sea, the Flemish Cap, the Tail of the Grand Banks, and finally, Line W (see Figures 3-10a and d). The layer thickness of the uLSW increases after the intense deep convection of the early 1990 throughout the subpolar gyre. This increase in layer thickness is also seen at Line W. The moored array measured a large range of uLSW layer thicknesses, as shown in Figure 3-11, and the variability at each mooring appears to be independent of the others. There are no clear patterns in layer thickness at BATS and Hydrostation S, and there is a large range of layer thickness values measured during the monthly/bi-weekly hydrographic surveys. Note that in the Flemish Pass, using our water mass definitions, the lower boundary of uLSW layer is the $1200 \mathrm{~m}$ deep sill. Since the upper boundary of uLSW gets deeper with time in the Flemish Pass, this leads to a decrease in layer thickness of uLSW in the Flemish Pass.

The thickness of the dLSW layer in the central Labrador Sea reached values of over $2000 \mathrm{~m}$ in the early 1990s then decreased throughout the 2000s, as shown in Figure 3-10b. The other subpolar datasets similarly show this decreasing trend, but with smaller layer thicknesses; the layer thickness of dLSW decreases when it is exported to the boundary current as it competes for space in the water column with other water masses. This layer thickness difference between the central Labrador Sea and the boundary was documented in Palter et al. (2007), for example, using (profiling) autonomous Lagrangian circulation explorer floats. The mechanism by which this layer thickness gradient is maintained was explained dynamically in Straneo (2006). Using an idealized model, Straneo (2006) finds a balance between convection in the interior, which maintains the layer thickness gradient between the boundary and the interior, and eddies, which drive exchange between the boundary and the interior through their tendency to eliminate gradients in layer thickness. So, the layer thickness of convectively formed water is thinner in the boundary current in steady state and eddy exchanges lead to water mass properties in the boundary that are similar to those in the interior.

The dLSW layer thickness at Line W decreases past its peak value in 1996; the moored array measured a statistically significant decrease in layer thickness as reported in Table 3-2 and shown in Figure 3-11. Apart from a slight increase in layer thickness in the early 2000s, there are no evident changes in dLSW layer thickness at BATS and Hydrostation S and there is generally a large range of measured layer thicknesses on short time scales. 

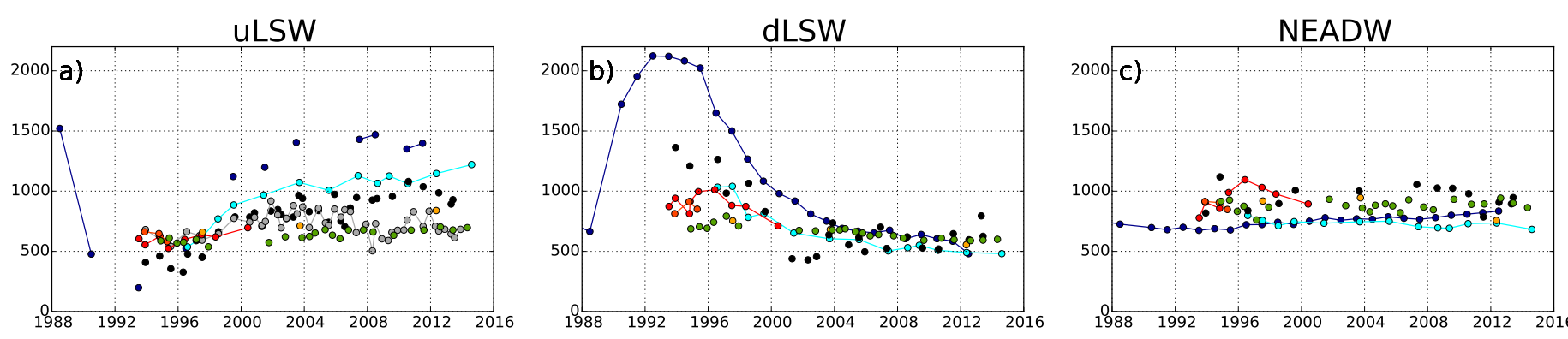

- Central Labrador Sea

- DWBC at 53N

- DWBC at the Flemish Cap

- DWBC at the Flemish Pass

- Tail of the Grand Banks (track 1)

- Tail of the Grand Banks (track 2)

- Tail of the Grand Banks (track 3)
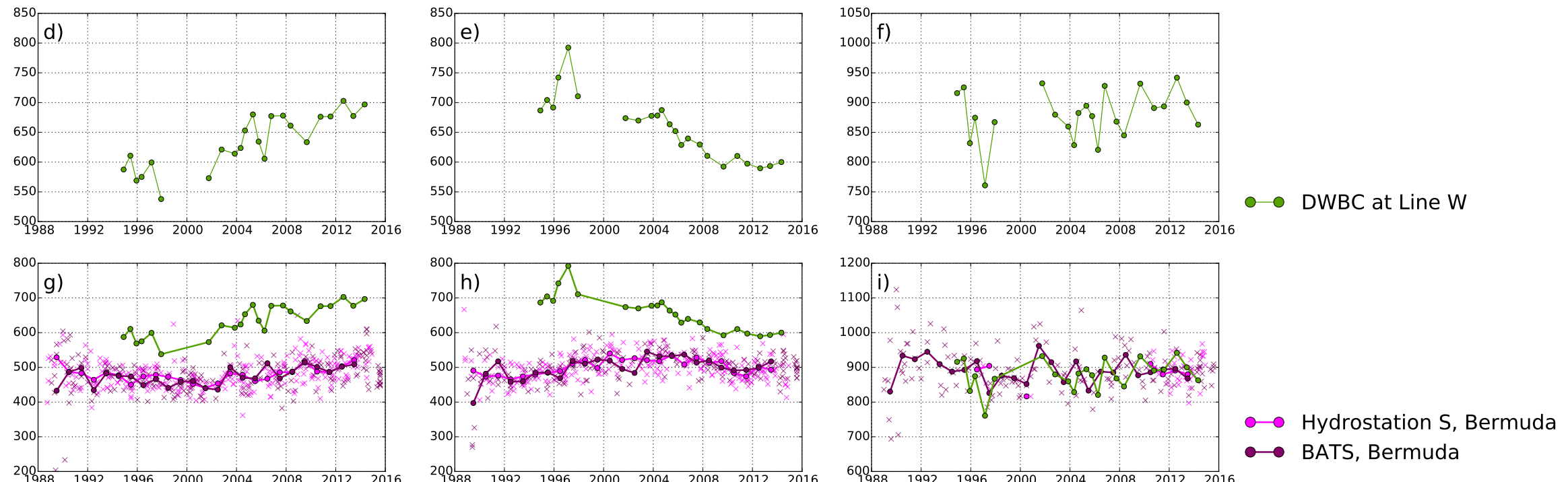

Figure 3-10: As in Figure 3-8 but for water mass layer thickness. Layer thickness ranges are consistent across rows for ease of comparison. uLSW layer thicknesses in the Labrador Sea are not shown when the surface water is denser than the upper boundary of the water mass. The dLSW is thickest in the early 1990s in the Labrador Sea when there was intense convection. Throughout the late 1990s and 2000s, this layer thins. A similar pattern is observed at Line W, with maximum thickness in 1997 and decreasing thickness throughout the 2000s. 


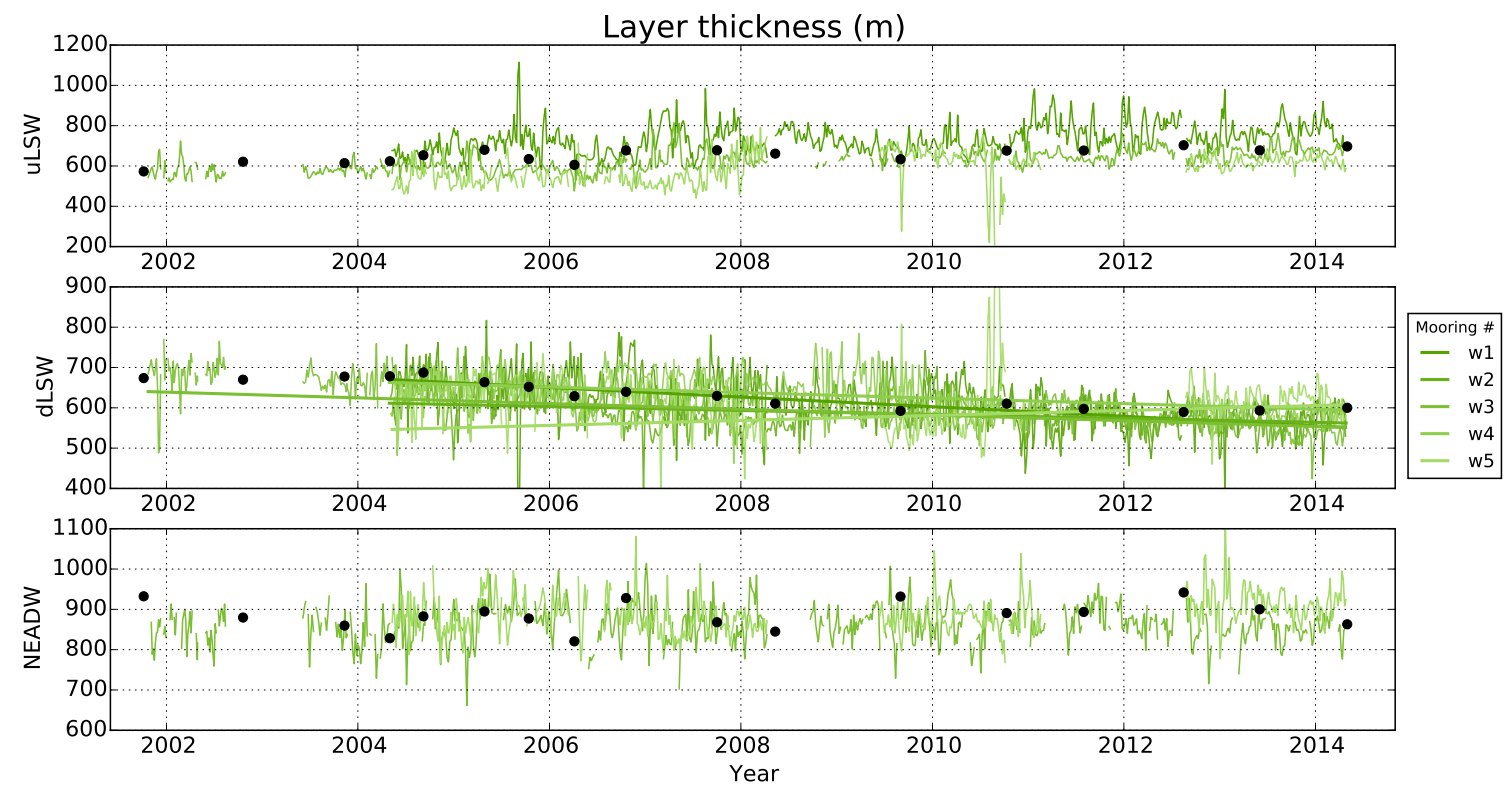

Figure 3-11: As in Figure 3-9 but water mass layer thickness as measured by Line W moorings. dLSW layer thickness decreases significantly at all moorings except w5, which is disrupted by a large event in late 2010. Layer thickness trends are quoted in Table 3-2.

The variability in the NEADW layer thickness in the subpolar gyre (see Figure 3-10c) is much smaller than layer thickness changes in either one of the LSW layers and there is no clear correspondence between layer thickness changes along the path of the DWBC. Because NEADW is not convectively formed, layer thickness changes in this layer are not as informative as in the dLSW layer. Further, NEADW layer thickness is dependent on how the width of the DWBC is defined. Because of the changing nature of the DWBC along its path, this is challenging to do consistently.

\section{Propagation of dLSW properties along the DWBC}

The dLSW layer in the Labrador Sea has a salinity minimum and layer thickness peak associated with the intense convection of the early 1990s. This event can be tracked throughout the subpolar gyre, appearing to arrive at Line W about 5 years later in 1997. While the dLSW salinity is very consistent from the central Labrador Sea to the Flemish Cap, it is altered by the time it reaches the Tail of the Grand Banks and Line W, indicating that significant stirring occurs south of the Flemish Cap, as suggested by Bower et al. (2009). 


\begin{tabular}{|l|c|c|c|c|c|}
\hline & $\mathrm{w} 1$ & $\mathrm{w} 2$ & $\mathrm{w} 3$ & $\mathrm{w} 4$ & $\mathrm{w} 5$ \\
\hline $\begin{array}{l}\text { Salinity trend } \\
(/ \text { decade })\end{array}$ & $0.012 \pm 0.002$ & $0.016 \pm 0.002$ & $0.014 \pm 0.002$ & $0.012 \pm 0.003$ & $0.012 \pm 0.002$ \\
\hline $\begin{array}{l}\theta \text { trend } \\
\left({ }^{\circ} \text { C/year }\right)\end{array}$ & $0.016 \pm 0.002$ & $0.018 \pm 0.002$ & $0.013 \pm 0.001$ & $0.010 \pm 0.002$ & $0.010 \pm 0.002$ \\
\hline $\begin{array}{l}\text { Layer thickness } \\
\text { trend }(\text { m/year })\end{array}$ & $-12 \pm 3$ & $-5 \pm 2$ & $-7 \pm 2$ & $-7 \pm 3$ & $\mathrm{n} / \mathrm{a}$ \\
\hline
\end{tabular}

Table 3-2: Trends and 95\% confidence intervals in dLSW water mass properties measured by Line W moorings from April 2004 - May 2014 (November 2001 - May 2014 for w3.)

\subsection{Quantification of water mass transit time distributions}

\subsubsection{Introduction to model framework}

In order to assess whether the Labrador Sea and Line $\mathrm{W}$ water mass property time series are consistent with realistic advective and mixing time scales, we use the Waugh and Hall (2005) boundary current model framework. In the Waugh and Hall (2005) model, tracers with concentration $\chi$ are advected along a boundary current with a constant speed, $u$, and are mixed with a stagnant interior at a constant rate with time scale, $t_{m i x}$. The simplified equations that describe their model are

$$
\begin{gathered}
\frac{\partial \chi_{b}}{\partial t}+u \frac{\partial \chi_{b}}{\partial x}+\frac{1}{t_{m i x}}\left(\chi_{b}-\chi_{i}\right)=S_{b} \\
\frac{\partial \chi_{i}}{\partial t}-\frac{\alpha}{t_{m i x}}\left(\chi_{b}-\chi_{i}\right)=S_{i}
\end{gathered}
$$

where the subscript $b$ denotes the property on the boundary and $i$ in the interior; $x$ is the along-boundary current coordinate; $\alpha$ is the ratio of the widths of the boundary and interior regions; $S$ indicates a source term. Both $\chi_{b}$ and $\chi_{i}$ are functions of $x$ and $t$.

A transit time distribution for a property propagating in the boundary can be associated with this model by finding the boundary Green's function, or solving equations 3.1 and 3.2 with a delta function boundary condition in the boundary current and no sources, i.e. $\chi_{b}(0, t)=\delta(t), S_{b}=S_{i}=0$, as described in Waugh and Hall (2005). Note that this transit time distribution is more precisely a measure of how the system dilutes a delta function signal at the origin, not a true expression of particle paths, but we use the term transit time distribution to connect with the literature and because it is a useful analogy. The ratio of the mixing time scale $t_{m i x}$ and the advective time scale $\left(t_{a d v}=L / u\right)$ shapes the transit 
time distribution; this ratio is the Peclet number, $P=t_{m i x} / t_{a d v}$. For $P \gg 1$, advection dominates over mixing and the transit time distribution approaches a delta function centered at $t_{a d v}$ as $P \rightarrow \infty$. For $P \ll 1$, mixing dominates and tends to broaden the transit time distribution i.e. more water parcels will appear to take longer to travel down the DWBC as they are mixed with the interior. The transit time distributions in these two limits are shown in Figure 3-12.

In the next two subsections, we will gauge the consistency of the Labrador Sea and Line W water mass property time series with the Waugh and Hall (2005) model and calculate transit time distributions in two different ways: we will use an analytical solution for periodic tracer signals and a forward model approach. These methods have complementary strengths and shortcomings and together show that the dLSW data are consistent with advective and mixing time scales which are on the same order.

\subsubsection{Analytical model}

One way to assess whether the Labrador Sea and Line W time series are consistent with the model framework is to use an analytical solution that Waugh and Hall (2005) derive for a tracer with periodic boundary conditions. In order to apply this analytical solution, we assume that the water mass temperature, salinity and layer thickness time series are periodic. We fit sinusoids to the data and assume that their entire time history are well represented by this fit. We can then use the analytical solution to find advective and mixing time scales between the Labrador Sea and Line $\mathrm{W}$ based on the relative amplitude and phase of the sinusoidal fits to each data set. As shown in Figure 3-13, the water mass temperature and salinity data do have a periodic signature to first order and there is evidence that they vary periodically on longer time scales (Hurrell and Deser, 2010).

The relative amplitude of two periodic time series separated by a set distance along the boundary current in this model is

$$
A_{\omega}=\exp \left(\frac{-t_{\omega}}{t_{\operatorname{mix}}} \times \frac{r_{\omega}^{2}}{\alpha(\alpha+1)+r_{\omega}^{2}}\right) \text { where } r_{\omega}=2 \pi t_{m i x} / T_{\omega}
$$

where $t_{\omega}$ is the phase lag between the two time series and $T_{\omega}$ is the period of oscillation of both time series. The upstream boundary conditions that lead to this solution are $\chi_{b}(0, t)=$ $\chi_{i}(0, t)=\operatorname{Re}\left(e^{i 2 \pi t / T_{\omega}}\right)$. This expression, which results from rearranging equations 10 and 11 in Waugh and Hall (2005), indicates that the amplitude of an oscillating time series will decrease more as the phase lag from the original signal increases. The dependence of the relative amplitude on $t_{m i x}$ and $T_{\omega}$ is more complicated, as illustrated in Waugh and Hall's Figure $6 \mathrm{~b}$. When $r_{\omega} \gg 1$ (or $t_{\text {mix }} \gg T_{\omega}$ ) the amplitude decays more slowly for larger $t_{m i x}$, 
i.e. less mixing. As can be deduced from Waugh and Hall's equation 10,

$$
t_{\omega}=t_{a d v} \frac{\alpha(\alpha+1)+r_{\omega}^{2}}{\alpha^{2}+r_{\omega}^{2}}
$$

in this limit, $t_{\omega} \approx t_{a d v}$. In other words, when the mixing time scale is much larger than the period of the oscillation, which we are assuming is larger or on the order of the advective time scale, then the signal's amplitude is preserved and phase shifted by the advective time scale, as illustrated in Figure 3-12a. The transit time distribution in this limit is the delta function centered at $t_{a d v}$.

The result in the opposite limit $\left(r_{\omega} \ll \alpha\right)$ is less intuitive: the amplitude decays more slowly for smaller $t_{m i x}$. This means that if mixing occurs quickly enough, the amplitude of a signal is preserved as it propagates. However, as can be derived from equation 3.4, the phase is shifted by $t_{\omega} \approx t_{a d v}(1+1 / \alpha)=\Gamma$, which, for $\alpha=0.1$, for example, is $t_{\omega} \approx 11 \times t_{a d v}$. In this limit, the transit time distribution is broad with a maximum at $\Gamma$, as shown in Figure 3-12b.

In between these two limits is the "intermediate mixing regime", in which the transit time distribution is a combination of a delta function with a decreased magnitude and a tail of longer transit times, as shown in Figure 3-12c. In this intermediate regime, the signal is attenuated as it propagates downstream and is phase shifted by a value between $t_{a d v}$ and $\Gamma$, depending on its period.

Rearranging Equation 3.3 gives a quadratic equation for $t_{m i x}$,

$$
t_{m i x}^{2}+\frac{t_{\omega}}{\ln \left(A_{\omega}\right)} t_{m i x}+\frac{\alpha(\alpha+1)}{\left(2 \pi / T_{\omega}\right)^{2}}=0
$$

Because $t_{m i x}$ is the solution to a quadratic equation, there are either zero, one, or two possible values of $t_{m i x}$ for a given set of parameters. Specifically, if $\left(\frac{t_{\omega}}{\ln \left(A_{\omega}\right)}\right)^{2}-\frac{\alpha(\alpha+1) T_{\omega}^{2}}{\pi^{2}}<0$, there is no solution for $t_{\text {mix }}$. This can be understood in the context of the two limits discussed above. Because $A_{\omega} \rightarrow 1$ for both $t_{m i x} \ll T_{\omega}$ and $t_{m i x} \gg T_{\omega}$, there is a minimum relative amplitude between the two limits; for any given combination of $T_{\omega}$ and $t_{\omega}$, there is a minimum $A_{\omega}$ that is possible.

To diagnose $t_{m i x}$ and $t_{a d v}$ and hence a transit time distribution between the Labrador Sea and Line W for dLSW and NEADW, we fit sinusoids to the central Labrador Sea potential temperature, salinity and layer thickness time series, shown from 1960 in Figure 3-13. As documented in Yashayaev (2007), there is a large change in water mass properties in the Labrador Sea from the late 1960s to the late 1990s. We also fit sinusoids to the time series from the start of deep convection in 1990 onward, as this event has a distinct signature and is the likely source of the signal we measured downstream at Line W. We then fit sinusoids with the periods that we found from fitting the Labrador Sea data to the Line W dLSW and NEADW potential temperature, salinity and layer thickness time series. From these 


\begin{tabular}{|c|c|c|c|c|c|c|}
\hline & \multicolumn{6}{|c|}{ dLSW } \\
\hline & $\theta_{\text {full }}$ & $S_{\text {full }}$ & $L T_{\text {full }}$ & $\theta_{1990-}$ & $S_{1990-}$ & $L T_{1990-}$ \\
\hline Fit parameters & & & & & & \\
$T_{\omega}$ : period (years) & 47 & 47 & 38 & 35 & 40 & 35 \\
$A_{\omega}$ : relative amplitude & 0.26 & 0.32 & 0.14 & 0.21 & 0.28 & 0.09 \\
$t_{\omega}$ : phase shift (years) & 4.2 & 4.8 & 2.4 & 5.8 & 7.3 & 5.9 \\
\hline Solutions & & & & & & \\
$t_{\text {mix }}$ (years) & $\mathrm{x}$ & $\mathrm{x}$ & $\mathrm{x}$ & $1.4 / 2.3$ & $0.9 / 4.8$ & $\mathrm{x}$ \\
$t_{\text {adv }}($ years $)$ & $\mathrm{x}$ & $\mathrm{x}$ & $\mathrm{x}$ & $2.4 / 3.8$ & $1.7 / 6.3$ & $\mathrm{x}$ \\
$u($ cm/s $)$ & $\mathrm{x}$ & $\mathrm{x}$ & $\mathrm{x}$ & $5.5 / 3.5$ & $8 / 2$ & $\mathrm{x}$ \\
$P=t_{\text {mix }} / t_{\text {adv }}$ & $\mathrm{x}$ & $\mathrm{x}$ & $\mathrm{x}$ & $0.58 / 0.62$ & $0.53 / 0.76$ & $\mathrm{x}$ \\
\hline
\end{tabular}

Table 3-3: Parameters of sinuisoidal fits to mean dLSW properties and resulting solutions with $\alpha=0.1$. $\theta$ refers to the dLSW potential temperature time series, $S$ to its salinity, and $L T$ to its layer thickness. The subscript full denotes fits to the full Labrador Sea time series and 1990- a fit to the data from 1990 onward.

fits we have estimates of $T_{\omega}$, the period of oscillation found from the Labrador Sea data fit, $A_{\omega}$, the ratio of the amplitude of the sinusoids fit to the Line $\mathrm{W}$ data and to the Labrador Sea data, and $t_{\omega}$, the phase shift between them. Note that because we are fitting a sinusoid with a set period to the data, the dLSW salinity minimum of the sinusoidal fit falls within the Line W data gap, unlike in Section 3.5 in which we linearly interpolated over the data gap.

Solving for $t_{m i x}$ and $t_{a d v}$ requires a choice of $\alpha$, the ratio of the width of the boundary current to the interior. Doney and Jenkins (1994) found a range of $\alpha$ from 0.03 to 0.13 using a numerical model to match measured tritium and ${ }^{3} \mathrm{He}$ propagation in the North Atlantic. Smith et al. (2016) estimate $\alpha=0.1$ from CFC-11 decay scales at Line W. For consistency with Smith et al. (2016) and Waugh and Hall (2005), we focus on $\alpha=0.1$.

The sinuisodal fits to the dLSW data have periods ranging from 35 to 47 years, relative amplitudes from 0.21 to 0.32 and phase shifts from 4.2 to 7.3 years, as detailed in Table 3-3 and shown graphically in Figure 3-14. We use equations 3.4 and 3.5 to solve for $t_{m i x}$ and $t_{a d v}$ for each set of parameters. 


$$
T_{\omega}=20 \text { years, } t_{a d v}=5 \text { years }
$$

a) Low mixing, $t_{m i x}=500$ years $\gg T_{\omega} / t_{a d v}, P \gg 1$
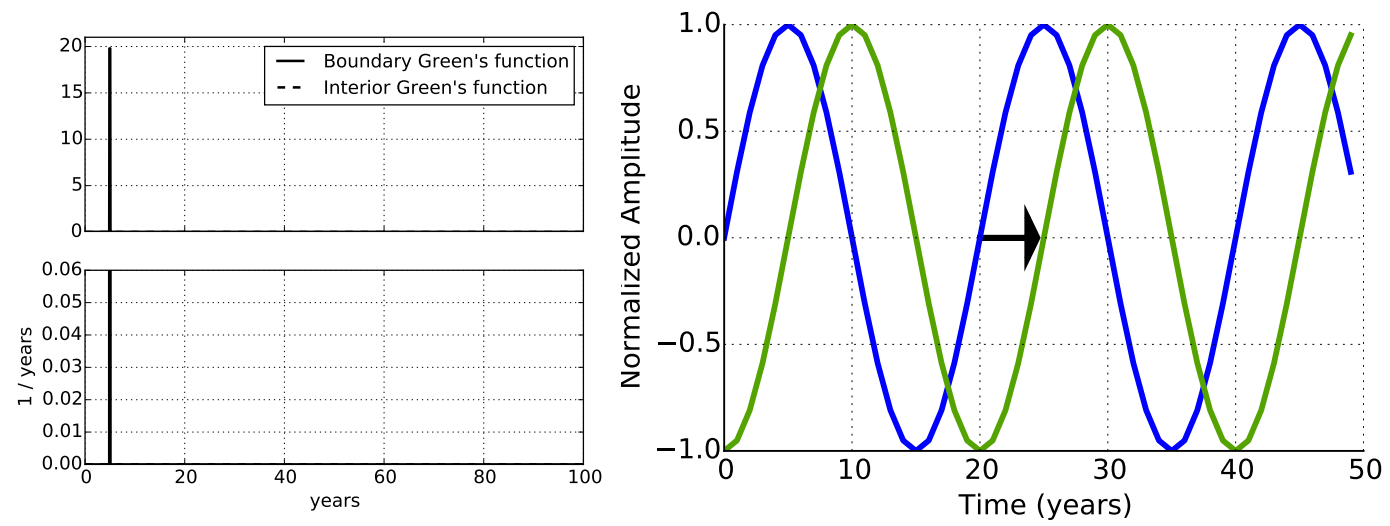

b) High mixing, $t_{m i x}=0.1$ years $\ll T_{\omega} / t_{a d v}, P \ll 1$
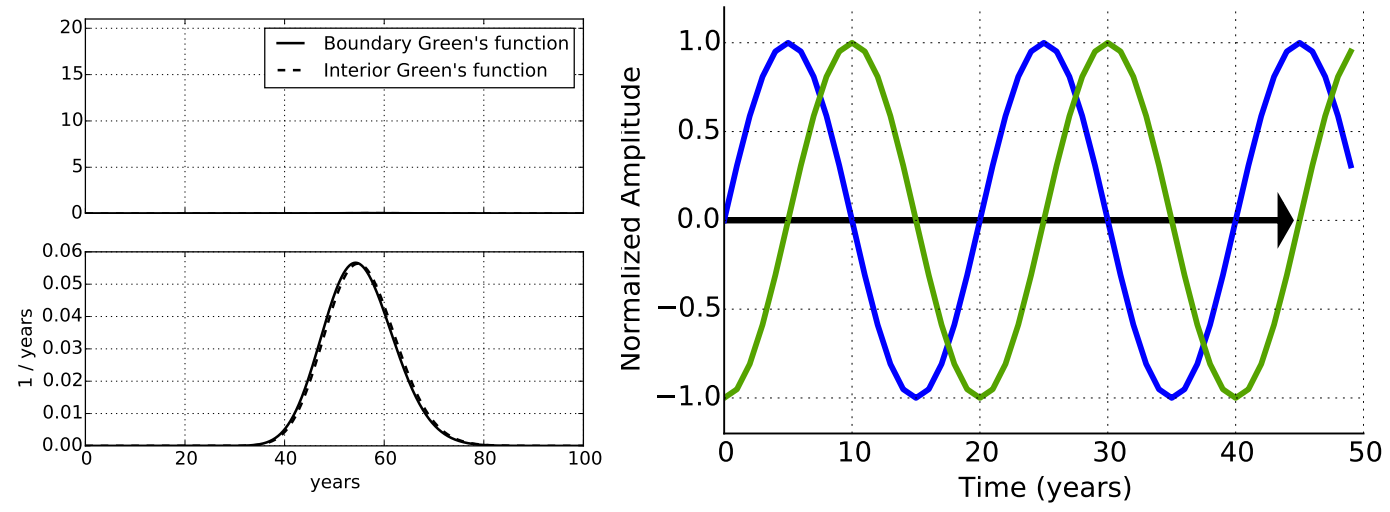

c) Intermediate mixing, $t_{m i x}=5$ years $=t_{a d v}, P \approx 1$
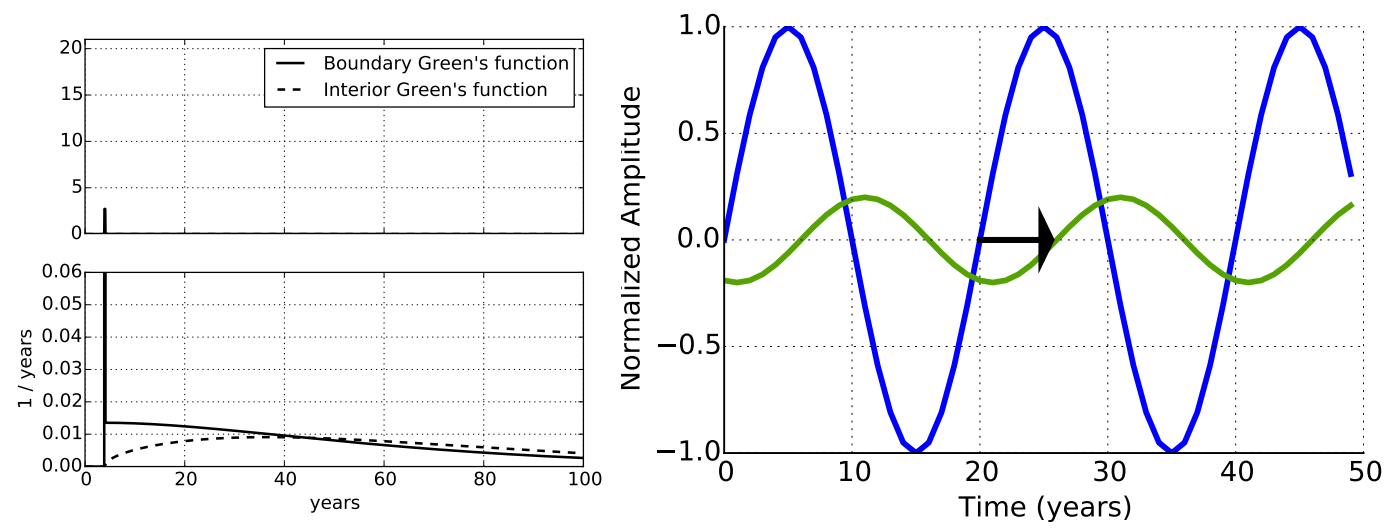

Figure 3-12: Transit time distribution and sample sinusoidal signals for different limits of the Waugh and Hall (2005) model; a) low mixing, b) high mixing and c) intermediate mixing. In each scenario, we consider an initial signal in the Labrador Sea with a period, $T_{\omega}$, of twenty years, depicted in blue, and a final signal, representative of Line $\mathrm{W}$ measurements, in green. The advective time scale between the two, $t_{a d v}$, is 5 years. The phase shift, $t_{\omega}$, is highlighted by the black arrow. In the low mixing limit, a) $t_{\omega}=t_{a d v}=5$ years. In the high mixing limit, b) $t_{\omega}=45$ years. Finally, in the intermediate limit, c) $t_{\omega}=6$ years, and the amplitude of the signal is most affected. 

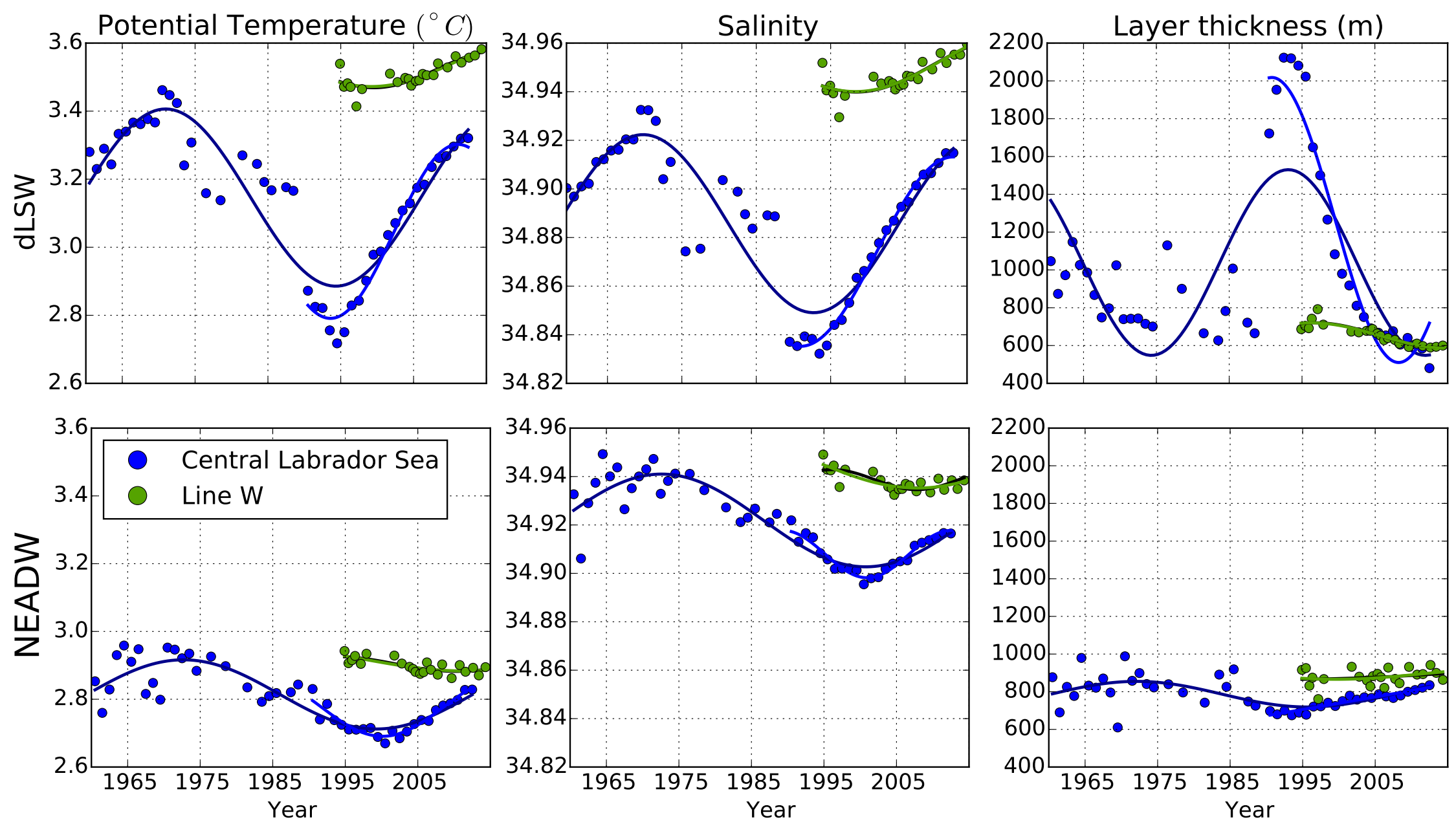

Figure 3-13: Sinusoidal fits to central Labrador Sea and Line W mean dLSW (top-row) and NEADW (bottom-row) potential temperature (left), salinity (center) and layer thickness (right). Blue dots are central Labrador Sea data points and green dots are Line W shipboard data points. Dark blue lines are sinuisoidal fits to all Labrador Sea data and light blue lines are sinusoidal fits from 1990 onward, when intense deep convection began in the Labrador Sea. Sinusoidal fits to Line W data are done by imposing the period from the Labrador Sea fits and the two results are indistinguishable here. 


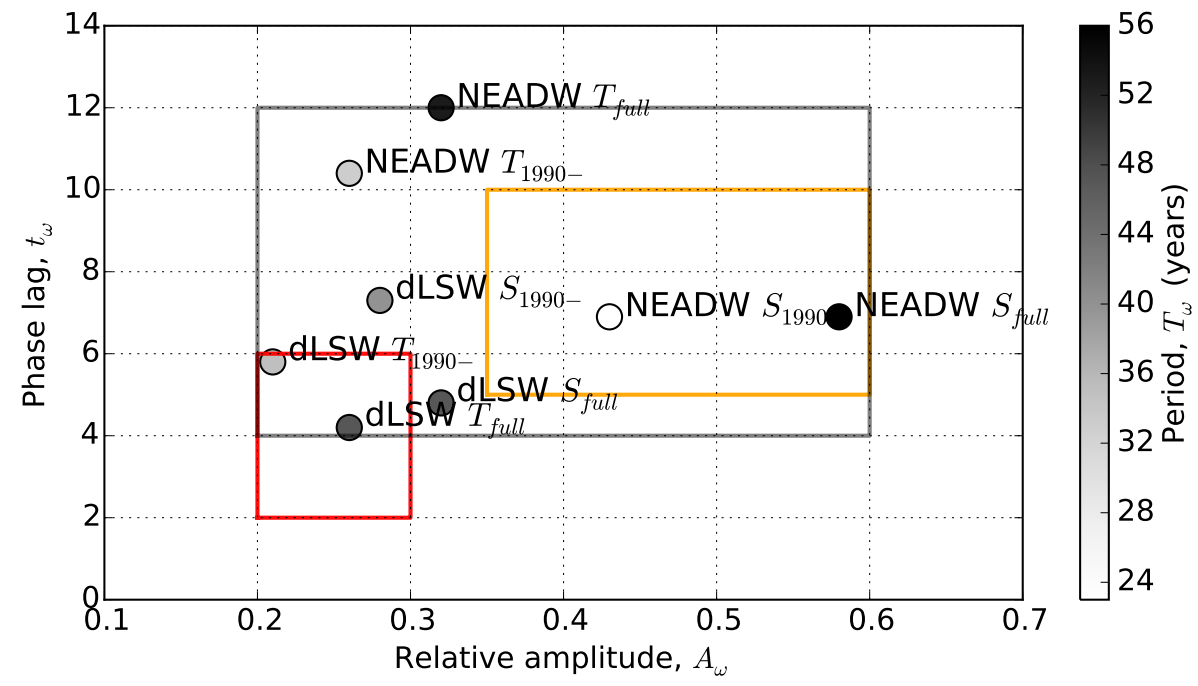

Figure 3-14: Parameter range of sinusoidal fits to Labrador Sea and Line $\mathrm{W}$ temperature and salinity data shown in relative amplitude and phase lag space. $T$ refers to a potential temperature time series and $S$ to a salinity time series. The subscript $f u l l$ denotes parameters from fits to the full Labrador Sea time series and 1990 - from a fit to the data from 1990 onward. Each point is shaded by the period of the sinusoidal fit as represented in the colorbar. The grey box highlights the range of parameters explored in the analysis shown in Figure 3-15; all periods in the colorbar were also included in this parameter space. The red box represents the spread of relative amplitude and phase lag found in the lagged correlation analysis shown in Figure 3-7 for dLSW and the orange box is the same for NEADW.

The choice of $\alpha$, the ratio of the width of the boundary current to the interior affects whether a solution can be found for a given set of parameters. For $\alpha=0.1$, only the fits to the dLSW potential temperature and salinity data from 1990 onward yield a consistent set of solutions; the combination of longer period and smaller phase lag in the fits to the full Labrador Sea data record act together to eliminate solutions with consistent $t_{m i x}$ and $t_{a d v}$. There are no consistent solutions for sinusoidal fits to either dLSW layer thickness time series. This is because the amplitude of the layer thickness signal decreases more between the central Labrador Sea and Line W than the potential temperature and salinity signals do. Smaller $\alpha$ can yield solutions for the full record, but none of the combinations of parameters yield consistent solutions for $\alpha=0.25$.

The sinusoidal fits to the NEADW data are less constrained than the dLSW fits, with periods ranging from 23 to 56 years, relative amplitudes from 0.26 to 0.58 and phase shifts from 6.9 to 12.0 years. All results are quoted in Table 3-4 and shown graphically in Figure 3 -14. There is a larger range of parameters in the NEADW layer than in the dLSW layer because the signals are smaller. Though the range of parameters is large and the solutions are more uncertain, because the relative amplitude and phase shifts for the NEADW fits are larger than the dLSW fits, there do exist solutions for all combinations of fit parameters to temperature and salinity time series for $\alpha=0.1$. 


\begin{tabular}{|c|c|c|c|c|c|c|}
\hline & \multicolumn{7}{|c|}{ NEADW } \\
\hline & $\theta_{\text {full }}$ & $S_{\text {full }}$ & LT $T_{\text {full }}$ & $\theta_{1990-}$ & $S_{1990-}$ & $L T_{1990-}$ \\
\hline Fit parameters & & & & & & \\
$T_{\omega}$ : period (years) & 53 & 56 & 47 & 33 & 23 & $\mathrm{x}$ \\
$A_{\omega}$ : relative amplitude & 0.32 & 0.58 & 0.42 & 0.26 & 0.43 & $\mathrm{x}$ \\
$t_{\omega}$ : phase shift (years) & 12.0 & 6.9 & 3.9 & 10.4 & 6.9 & $\mathrm{x}$ \\
\hline Solutions & & & & & & \\
$t_{\text {mix }}$ (years) & $0.82 / 9.7$ & $0.75 / 11.8$ & $\mathrm{x}$ & $0.43 / 7.2$ & $0.19 / 7.8$ & $\mathrm{x}$ \\
$t_{\text {adv }}($ years $)$ & $1.9 / 11.2$ & $1.0 / 6.5$ & $\mathrm{x}$ & $1.5 / 9.8$ & $0.78 / 6.76$ & $\mathrm{x}$ \\
$u(\mathrm{~cm} / \mathrm{s})$ & $1.2 / 6.9$ & $13.2 / 2.0$ & $\mathrm{x}$ & $9.0 / 1.4$ & $17.1 / 1.97$ & $\mathrm{x}$ \\
$P=t_{\text {mix }} / t_{\text {adv }}$ & $0.87 / 0.42$ & $0.75 / 1.80$ & $\mathrm{x}$ & $0.29 / 0.73$ & $0.24 / 1.16$ & $\mathrm{x}$ \\
\hline
\end{tabular}

Table 3-4: As in table 3-3 but for NEADW.

There is no solution to the sinusoidal fit to the full NEADW layer thickness time series as the relative amplitude and phase shift are smaller than the fits to the full potential temperature and salinity time series. The NEADW layer thickness time series from 1990 onward cannot be fit with a reasonable sinusoid. Indeed, the utility of this framework for the NEADW layer thickness is questionable as there is no layer thickness signature associated with NEADW.

To gauge the sensitivity of solutions for $t_{a d v}$ and $t_{m i x}$ to each of the parameters, we found solutions for the range of parameters relevant to the dLSW and NEADW potential temperature and salinity time series; this parameter range is shown in Figure 3-14. This sensitivity analysis, which is displayed graphically in Figure 3-15, shows that the period is not a significant control on the magnitude of the solutions for the parameter range we found, though it does affect whether a solution exists, with a smaller solution space for larger periods.

As explained above, there are typically two solutions for each combination of parameters, one which corresponds to fast advection and fast mixing and one for which advection and mixing are both slower. Because the directly measured speed of the DWBC is on the order of $5 \mathrm{~cm} \mathrm{~s}^{-1}$ (Toole et al., 2011; Schott et al., 2006; Fischer et al., 2015), and will only get slower with horizontal averaging, the slower advection and mixing solution is much more realistic. For these solutions, $t_{m i x}$ is most sensitive to the relative amplitudes of the Line $\mathrm{W}$ and Labrador Sea time series; smaller relative amplitudes indicate shorter $t_{m i x}$, or a larger role for mixing. $t_{a d v}$ is most sensitive to the phase lag between the time series, the longer the phase lag between oscillations, the longer the advective time scale. As shown in Figure 3-15, all of the solutions we consider realistic fall between $P=0.5$ and $P=2$.

The solutions to the set of NEADW parameters found here have a large spread and it is 
not possible to say anything conclusive about the transit time distribution of NEADW from the Labrador Sea and Line W, except that it is possible to find solutions and that there may be less of a role for mixing for this water mass, as hypothesized by Lozier et al. (2013). This uncertainty is unsurprising given the small amplitude of the NEADW water mass property variability in the Labrador Sea.

The dLSW parameters on the other hand, yield more tightly constrained solutions. The realistic solutions for the post 1990 dLSW potential temperature and salinity time series are $t_{a d v}=3.8$ and 6.3 years, $t_{m i x}=2.3$ and 4.8 years respectively, which give Peclet numbers $P=0.62$ and 0.77 . The transit time distributions that correspond to these parameters, shown in Figure 3-16, have a dominant peak at $t_{a d v}$ and a long tail of transit times that extends well into 100 years. As discussed in Waugh and Hall (2005), the height of the dominant peak determines the degree of attenuation of the signal as it propagates and the shape of the tail can shift its phase further than the advective time scale.

The analytical solution to the Waugh and Hall (2005) framework requires the assumption of regular periodicity of water mass properties. However, the water mass data are periodic to first order, and we are able to find consistent solutions for dLSW potential temperature and salinity evolution. The solutions indicate a first order role for both advection and diffusion in the DWBC between the Labrador Sea and Line W with an advective time scale on the order of 5 years. In the next subsection we describe an alternate approach to finding transit time distributions that does not require the assumption of regular periodicity. 

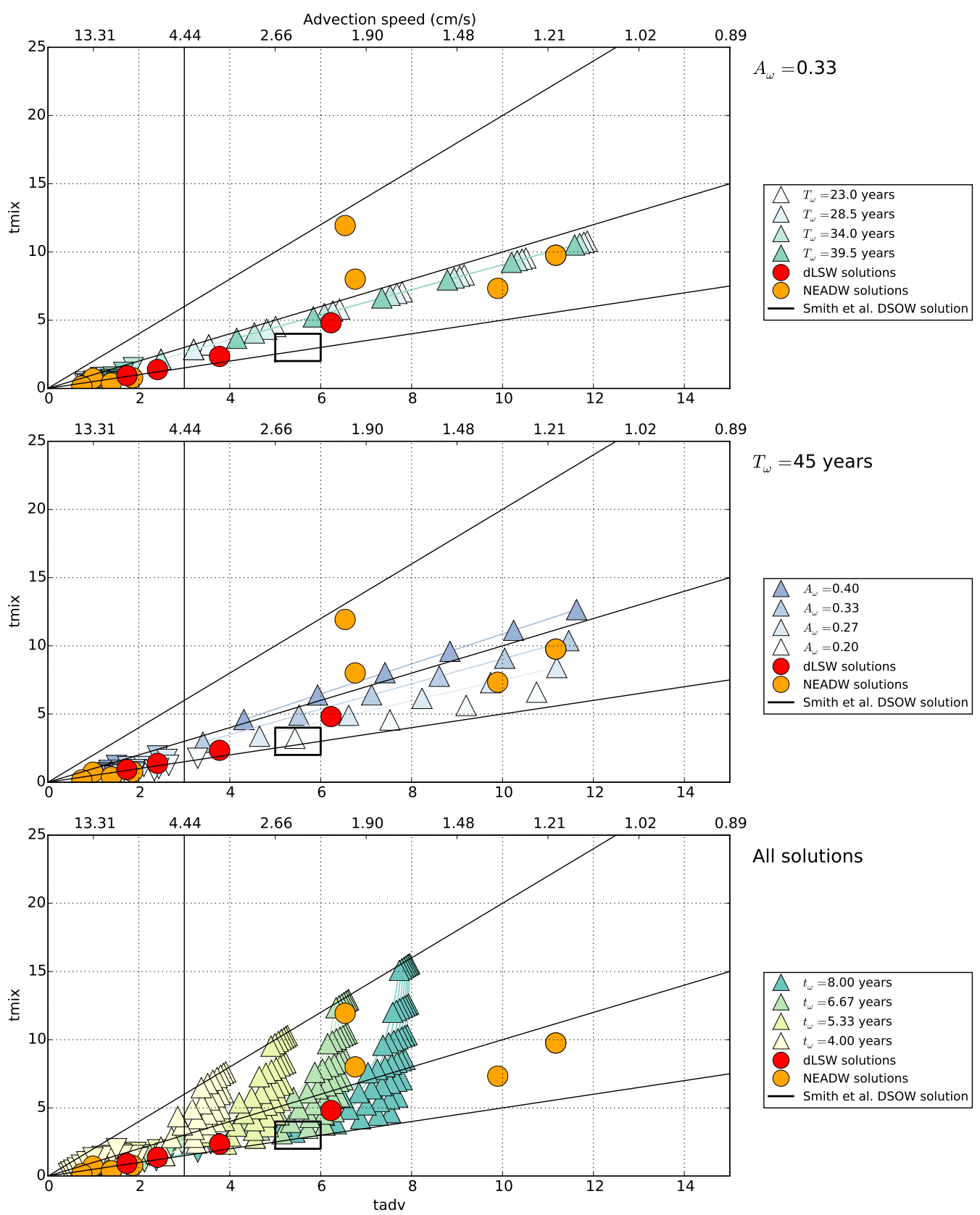

Figure 3-15: Solutions to parameter space sensitivity analysis. The diagonal black lines are the $P=0.5,1$ and 2 lines. In all panels, triangles pointing upwards are positive solutions and triangles pointing downwards are negative solutions. Vertical line at $t_{a d v}=3$ separates realistic (longer $t_{a d v}$ ) from unlikely solutions. The red points are solutions to the dLSW time series fit parameters and the orange are solutions to NEADW time series fit parameters. The black square indicates the range of solutions obtained by Smith et al. (2016) for DSOW from the Labrador Sea to Line W. Top panel: solutions for relative amplitude, $A_{\omega}=0.33$, with color representing different periods, showing that there is little sensitivity to period in this parameter range. Central panel: solutions for period, $T_{\omega}=45$ years, with color representing different relative amplitudes, which alters the mixing time scale solution significantly. Bottom panel: all solutions, with color representing different phase lags between sinusoidal fits; in general a longer phase lag between sinusoidal fits will yield a longer advective time scale. All solutions shown are for $\alpha=0.1$ 

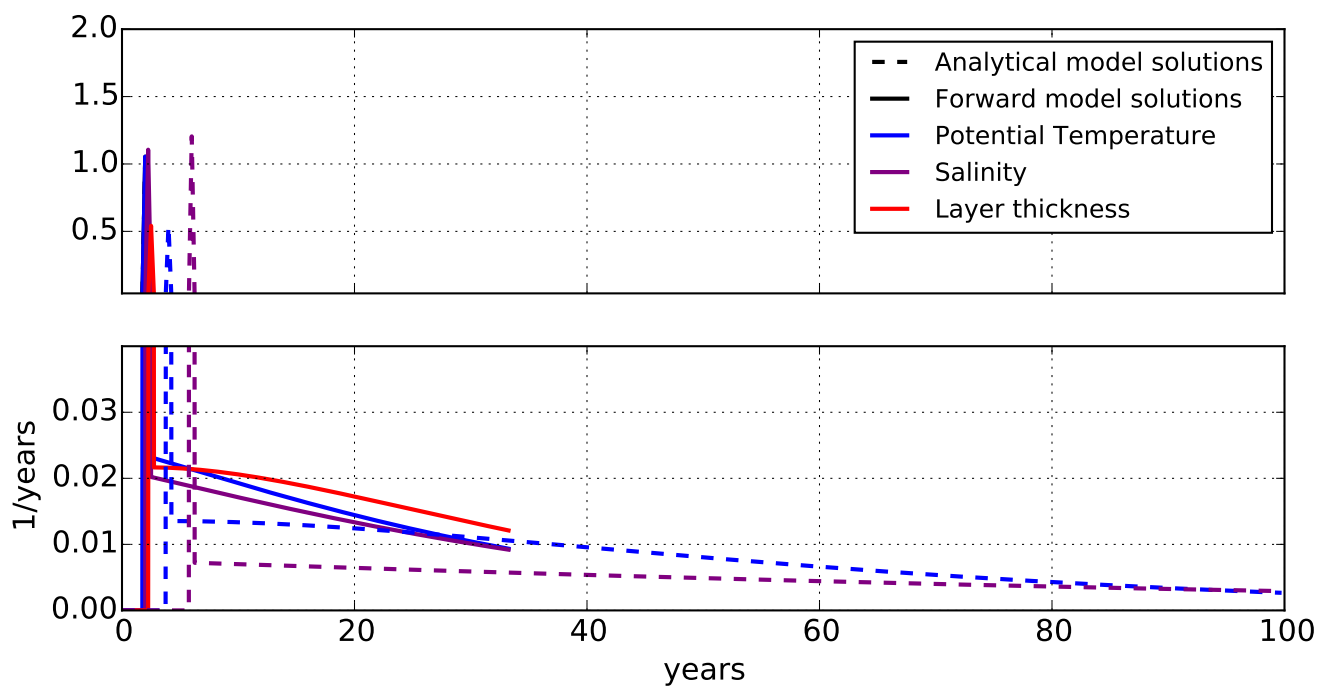

Figure 3-16: Range of potential transit time distributions solutions from the Labrador Sea to Line W for dLSW. Delta functions are normalized so that transit time distribution integrates to 1. Dashed lines correspond to analytical model solutions and solid lines to forward model solutions. Solutions for potential temperature, salinity and layer thickness time series are shown in blue, purple and red respectively. 


\subsubsection{Forward model}

An alternative approach to solving for the Green's function for the Labrador Sea and Line $\mathrm{W}$ is to apply a forward model to the Labrador Sea and assess how well it fits the Line W data. The advantage of this approach is that it does not require any assumptions about the shape of the data time series or its history before measurements were made. However, because the Labrador Sea dataset began 34 years before the Line W dataset, this limits the length of the transit time distribution solution to 34 years, as shown in Figure 3-16.

To find a modeled downstream property time series, we apply

$$
\chi_{b}(L, t)=\int_{0}^{T_{\max }} \chi_{b}(0, t-\xi) G(L, \xi) d \xi
$$

which is modified from Waugh and Hall (2005)'s equation 5 for a conserved tracer and a finite upstream time history. $\chi_{b}(0, t)$ is the upstream property time series, the central Labrador Sea in this case, and $\chi_{b}(L, t)$ is the downstream property time series, meant to model the property time series at Line W. $G(L, t)$ is the Green's function for properties at Line W, or transit time distribution between the Labrador Sea and Line W, which is the function we are solving for. $T_{\max }$ is the time over which the Green's function can be integrated, so in this case, it is the length of time that measurements were taken in the central Labrador Sea before the Line $\mathrm{W}$ measurements began, which is 34 years.

We apply equation 3.6 to the central Labrador Sea property time series with Green's functions solutions that correspond to a range of advective time scales and mixing time scales, $t_{a d v}$ and $t_{m i x}$. The functional form of the Green's function solution to equations 3.1 and 3.2 can be found in Waugh and Hall (2005). To assess how well the modeled downstream time series matches the Line $\mathrm{W}$ data, we calculate a cost function,

$$
J=\sum\left(\chi_{L W}(t)^{\prime}-\chi_{b}(L, t)^{\prime}\right)^{2}
$$

where $\chi_{L W}(t)^{\prime}$ is the property anomaly time series observed at Line $\mathrm{W}$ and $\chi_{b}(L, t)^{\prime}$ is the modeled anomaly time series. For both time series, the anomaly time series is the difference of the time series and its own time mean. We consider anomalies because there is an offset between the Labrador Sea and Line W potential temperature and salinity which we do not model. This is offset occurs because the interior is warmer and saltier than the boundary current. Because the values and distribution of interior properties are not well constrained, we focus on the evolution of the signal in the boundary current and how stirring affects the propagation of anomalies This amounts to neglecting how the mean properties are affected in finding our solution, but we do not hold the interior properties constant. Possible additions to the model to account for this offset are sources in the interior or an altered boundary condition for the interior at $x=0$, but this would add both complexity and uncertainty to 

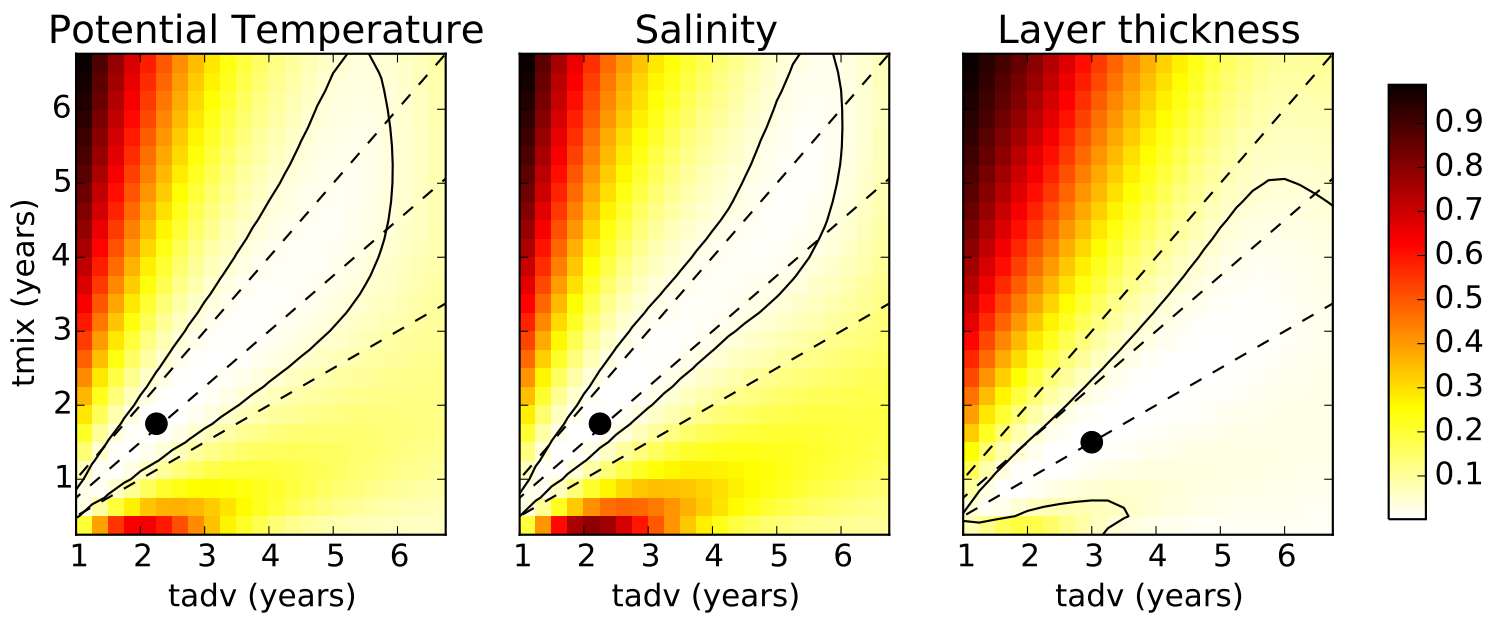

Figure 3-17: Normalized cost functions for forward model fits to Line W dLSW potential temperature, salinity and layer thickness time series, as a function of tadv and tmix. The black dot corresponds to the minimum cost function value for each property and the black contour highlights the $5 \%$ normalized cost function line. The dashed lines are the $\mathrm{P}=1, \mathrm{P}=0.75$ and $\mathrm{P}=0.5$ lines for reference.

our interpretation.

As in the analytical model approach, applying this method to NEADW properties does not yield consistent solutions, so we focus on dLSW properties. The cost functions for modeled dLSW potential temperature, salinity and layer thickness time series are consistently minimized for combinations of $t_{a d v}$ and $t_{m i x}$ that have Peclet numbers that are close to or less than one, as shown in Figure 3-17. Note that the cost functions have been normalized by the maximum cost function value that was found for the range of parameters explored for each property.

The advective and mixing time scales that minimize the cost function for dLSW temperature and salinity anomaly time series at Line $\mathrm{W}$ (with 0.25 year resolution) are $t_{a d v}=2$ and 2.25 years and $t_{m i x}=1.5$ and 1.75 years respectively, with Peclet numbers of 0.75 and 0.78. The Line $\mathrm{W}$ dLSW layer thickness cost function is minimized for $t_{a d v}=2.5$ years and $t_{m i x}=1.25$, or $P=0.5$. These results do not change significantly if a synthetic constant time history is added to extend the time over which the Green's function is integrated, or if single data points are removed from the Line $\mathrm{W}$ data set, i.e. no single data point is governing the fit. The result is most sensitive to removing the minimum dLSW potential temperature, salinity and maximum layer thickness measurement in February 1997. Even removing this point changes each of the time scales by less than one year, and all Peclet numbers are unchanged to within one decimal point.

The property anomaly time series modeled with the cost function minimizing parameters replicate the dLSW potential temperature and salinity minimum and layer thickness maximum observed at Line W in the late 1990's as well the slopes before and after this 


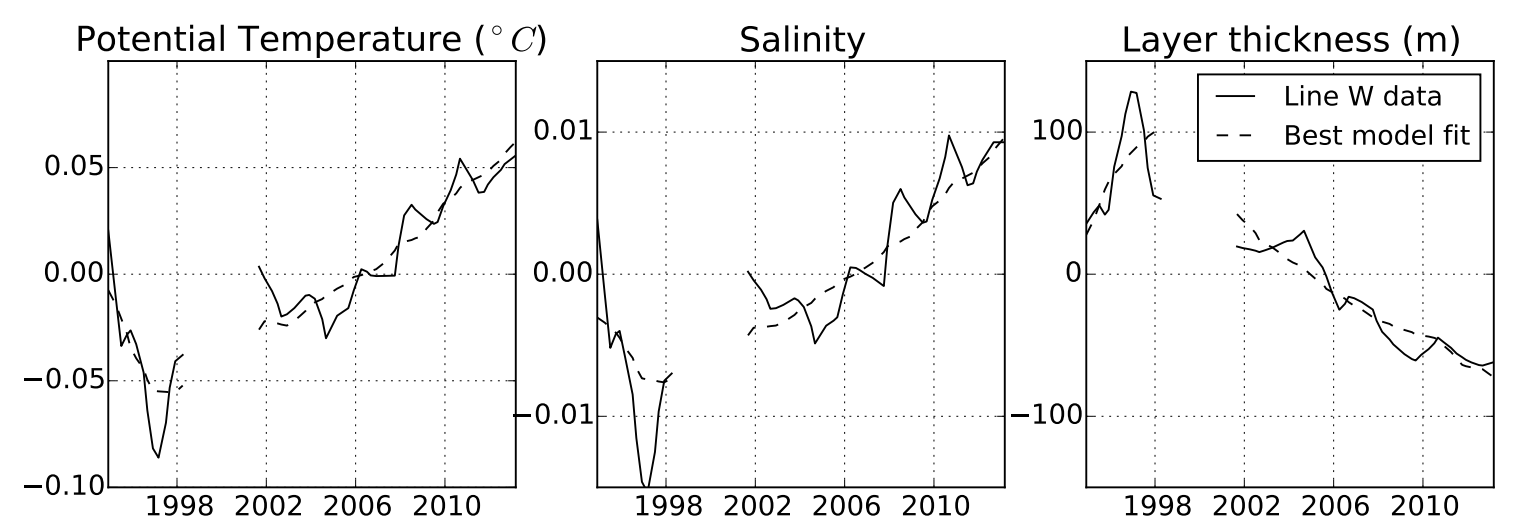

Figure 3-18: Best fits from forward model to Line W dLSW potential temperature, salinity and layer thickness anomaly times series. Solid lines are linearly interpolated Line W dLSW property anomaly time series and dashed lines are the property anomalies found by applying the best fit transit time distribution to Labrador Sea property time series. The best fit parameters, which minimize the cost function, are the black dots in Figure 3-17.

extreme deep convection signal, as shown in Figure 3-18. However, because there is a data gap between 1999 and 2001, there is uncertainty as to the timing of the arrival of the deep convection event, and these estimates of the advective time scale are likely biased short. Indeed, an advective time scale of 2 years corresponds to a speed of $7 \mathrm{~cm} \mathrm{~s}^{-1}$, which is faster than one would expect for a mean boundary current speed given direct velocity measurements of the DWBC. As can be seen in Figure 3-17, there is a range of combinations of $t_{a d v}$ and $t_{m i x}$ which minimize the cost function, and a combination with a longer $t_{a d v}$ is more realistic.

These results imply a greater role for mixing in the propagation of layer thickness anomalies than in the propagation of potential temperature or salinity anomalies. This is in line with our interpretation of neutral density layer thickness anomalies as less conservative than temperature and salinity anomalies on neutral density layers because layer thickness is limited by the depth of the water column.

The dLSW advective and mixing time scales between the Labrador Sea and Line W are likely underestimated by the forward model method because of the data gap from 1999 to 2001 at Line W. However, the form of the cost function confirms a similar order role for advection and mixing, as we found using the analytical solution. 


\subsection{Conclusions}

Observations of the DWBC at Line $\mathrm{W}$ reveal water mass changes that are consistent with changes upstream in the Labrador Sea, especially in the dLSW water mass associated with intense deep convection in the Labrador Sea in the early 1990s. We show shifts in potential temperature and salinity at the Line $\mathrm{W}$ moored array that are consistent with the cessation of intense deep convection in the Labrador Sea and quantify the coherence between water mass property time series in neutral density space. In this work, we define the dLSW layer as the portion of LSW whose salinity and layer thickness time series at Line W are highly lag-correlated with properties at the same neutral density in the Labrador Sea. We find that the dLSW defined in this way is representative of the water formed during the intense deep convection of the early 1990s. This strong correlation in water mass salinity and potential temperature time series also holds for the underlying NEADW layer, though with a longer lag time and higher uncertainty.

Further, we draw on additional datasets to explore the evolution of water mass properties along the path of the DWBC, using consistent neutral density water mass definitions throughout. We find that the dLSW layer salinity is very similar from the Labrador Sea to the Flemish Cap, but is increased at the Tail of the Grand Banks, and increased further at Line W. The salinity minimum associated with the intense deep convection of the early 1990s can be tracked throughout these datasets, though the magnitude of variability in the record decreases as the signal propagates downstream. These findings are consistent with Stramma et al. (2004), who track water mass properties traveling through the northern subpolar gyre coherently, as well with Bower et al. (2009), who find high amounts of stirring between the boundary and the interior at the Tail of the Grand Banks. We also show that the salinity minimum arrives at Line $\mathrm{W}$ before arriving at Bermuda. As Line $\mathrm{W}$ and Bermuda are both about $4500 \mathrm{~km}$ downstream from the Labrador Sea, this indicates that dLSW properties travel faster along the boundary than in the interior pathway to Bermuda, which is also consistent with other studies (Bower et al., 2009; Rhein et al., 2015; Smith et al., 2016).

Through a lagged correlation of dLSW potential temperature and salinity time series, we estimate a mean transit time between Line $\mathrm{W}$ and the Labrador Sea of 5 years, or a spreading rate of about $2.5 \mathrm{~cm} \mathrm{~s}^{-1}$ over a distance of $4500 \mathrm{~km}$ along the $3000 \mathrm{~m}$ isobath. There is significant uncertainty in this estimate because the shipboard measurements on which it is based are annual or semiannual. Further, because there is a three year data gap after the salinity minimum that we measure in 1997, this estimate may be biased fast; a fresher salinity minimum may have occurred during the 1999-2001 data gap.

Our estimate is faster than Peña-Molino et al. (2010)'s estimate of 9 years for dLSW, which rested primarily on the timing of PPV minima and was limited by the starting time of Line $\mathrm{W}$ mooring w3. Our estimate is consistent with other mean transit times along 
the boundary deduced from salinity anomalies on density surfaces; Molinari et al. (1998) and Van Sebille et al. (2011) both found a 10 year transit time for "classical" LSW from the Labrador Sea to the Abaco Line at $26.5^{\circ} \mathrm{N}$, corresponding a $2-2.5 \mathrm{~cm} \mathrm{~s}^{-1}$ spreading rate. However, Molinari et al. (1998) and Van Sebille et al. (2011) found ratios of water mass variability amplitude between the Abaco Line and the Labrador Sea of order 1 and 0.5 respectively. These amplitude ratios are larger than those we measure for dLSW salinity and temperature time series at Line $\mathrm{W}(\approx 0.2-0.3)$, which is upstream of Abaco. If the system can be modeled using an advective-diffusive model, it does not make sense that the signal would be larger at Abaco than at Line W. Van Sebille et al. (2011) suggest that the preservation could indicate that there is very high mixing between the boundary and the interior, i.e. that the DWBC is in the fast mixing, fast advection limit described in Section 3.7. However, this would require mean DWBC advection speeds that are faster than any DWBC velocities that have been recorded, on the order of $50 \mathrm{~cm} \mathrm{~s}^{-1}$. Strictly, our results cannot be compared directly because we have different water mass definitions. Further, it is unclear whether the Waugh and Hall (2005) model can be applied between the Labrador Sea and the Abaco line, because the DWBC has crossed underneath the Gulf Stream to get to Abaco. Some of the high salinity variance may be explainable through the influence of Mediterranean Overflow Water, and even Antarctic Intermediate Water (though it generally occupies lower densities). However, there does appear to be an inconsistency here that is not resolved.

Using the Waugh and Hall (2005) solution for the propagation of periodic signals in boundary currents, we are able to estimate the transit time distribution of water parcels in the dLSW density range arriving at Line W from the Labrador Sea. Using an analytical solution for periodic tracers, we find a range of mixing time scales of $t_{m i x} \approx 2.3-4.8$ years, and advective time scales of $t_{a d v} \approx 3.8-6.3$ years. However, estimating the transit time distribution using this analytical solution requires the assumption of a periodic time history, and only sinusoidal fits to the Labrador Sea data from 1990 onward yield physical results. Using a forward model applied to the Labrador Sea data yields $t_{m i x} \approx 1.25-1.75$ years and $t_{a d v} \approx 2-2.5$ years, which are likely underestimates due to the gap in the Line $\mathrm{W}$ data from 1999 to 2001. The dependence of the cost function on $t_{a d v}$ and $t_{m i x}$ (Figure 3-17) shows that longer advective and mixing time scales with similar Peclet numbers can also lead to solutions with small cost functions. These two methods yield consistent solutions with Peclet numbers between 0.6 and 0.8 for dLSW water mass properties, implying that advection and diffusion play leading order roles in the propagation of dLSW water mass property signals. These solutions imply less mixing than Waugh and Hall's $\left(t_{m i x} \approx 1\right.$ year) and hence a slower decay in amplitude along the path of the DWBC.

We can also make an estimate of the bulk diffusivity, $\kappa$, between the DWBC and the 
interior as it travels from the Labrador Sea to Line W,

$$
\kappa=\frac{l \times D}{t_{\operatorname{mix}}},
$$

where $l$ is the width of the DWBC and $D$ is the distance between the Labrador Sea and Line $\mathrm{W}$, as in Doney and Jenkins (1994). For $l=100 \mathrm{~km}, D=4500 \mathrm{~km}$ and $t_{m i x}=2.5$ years, $\kappa=5700 \mathrm{~m}^{2} \mathrm{~s}^{-1}$, which is elevated, but on the same order as the Ledwell et al. (1998) estimate of $\kappa=1000 \mathrm{~m}^{2} \mathrm{~s}^{-1}$ in the eastern North Atlantic, as we might expect, given the strong stirring along the western boundary of the North Atlantic.

Comparisons with transit times deduced from other tracers can be misleading, as Waugh and Hall (2005) explain. In the intermediate mixing regime, which the DWBC is likely in, the spreading rate inferred from lagged correlations of tracer time series depends on the tracer initial conditions. Anthropogenic tracers, which often have approximately exponential growth at the beginning of their time history, will be altered differently by a transit time distribution with a tail of long transit times than a tracer with a periodic time history, such as salinity on neutral density surfaces, which in the Labrador Sea tends to oscillate on (multi)decadal time scales in tune with the NAO. For a tracer that has exponential growth, the tail of long transit times will act to flatten out the exponential curve that you measure downstream and cause an overestimate of transit times if it is not analyzed carefully. Conversely, for an oscillatory salinity time series upstream, this tail of long transit times will bring anomalies of both signs, preserving the shape of the signal though potentially shifting the phase. The amplitude of the signal also decreases as it propagates downstream. Therefore, to measure transit times from salinity, a signal with a large amplitude is required upstream, such as the intense deep convection in the early 1990s. The advantage to diagnosing transit times from salinity when you can, is that measurements are more readily available and the effective propagation speeds are faster. The advantage to tracers with exponential time histories is that you can be confident of their high latitude source, as they have very low concentrations in the interior subtropical gyre. A full picture can be put together by considering results from both types of tracers.

Smethie (1993) used CFC measurements to predict an along-boundary spreading rate of $0.8 \mathrm{~cm} \mathrm{~s}^{-1}$ for the LSW CFC maximum from the Labrador Sea to Line W and $1-2 \mathrm{~cm} \mathrm{~s}$ for all DWBC water masses in a later study (Smethie et al., 2000). Doney and Jenkins (1994) estimated a DWBC Tritium and Helium spreading rate $0.75-1.5 \mathrm{~cm} \mathrm{~s}^{-1}$ from the Labrador Sea to the subtropical North Atlantic. These values are slower than our estimate likely because of stirring with water that has lower tracer concentration and because of recirculation, as these studies all recognize. As explained above, because these tracers have approximately exponential time histories at the source, spreading rate estimates are likely to be biased slow. 
Recently, Rhein et al. (2015) compiled 25 years of CFC data to make maps of age and fraction of young deep water. Their results imply a CFC transit time of 11 years for LSW to Line $\mathrm{W}$ along the boundary, which is, again, longer than our estimate. This is likely because their assumed transit time distribution does not have a tail of long transit times as we infer here. As they integrate many data sets, they are able to show that the spreading rate of LSW is not uniform throughout its path from the Labrador Sea to Line W. In fact, they show that spreading is slowest through the "transition zone" near the Flemish Cap, which is also indicated by our analysis of water mass properties along the path of the DWBC.

Smith et al. (2016) use the Waugh and Hall (2005) model to estimate $t_{a d v}$ and $t_{\text {mix }}$ for DSOW from the Labrador Sea to Line W using CFC and Iodine measurements. Their results, $t_{a d v} \approx 5-6$ years and $t_{m i x} \approx 2-4$ years are not directly comparable with ours, $t_{a d v} \approx 3.8-6.2$ years, $t_{m i x} \approx 2.3-4.8$ years, as our estimate is for dLSW and theirs is for DSOW, however our results agree well, indicating that there may not be much variation with depth in along-boundary water mass spreading rates of North Atlantic DWBC waters.

There are not many estimates of NEADW spreading into the subtropical gyre. Water mass property changes are smaller at these high densities and NEADW cannot be tracked using CFCs as there is a local CFC minimum with depth in the NEADW layer. In the high resolution FLAME model, Lozier et al. (2013) find that overflow waters in the DWBC experience less stirring than LSW, which is consistent with our findings. However, they also find that it propagates faster than LSW, which is contrary to our finding of a longer lag time in the NEADW layer. However, because the signals in the NEADW layer are smaller, there is higher uncertainty associated with our transit time estimates for NEADW.

Transit time distributions for LSW have been calculated using particle trajectories from several model products. Unlike the transit time distributions we infer, their transit time distributions are generally broad without an early advective peak. This is due to the difference in method used to find the transit time distribution. In assuming that the upstream and downstream signals have the same shape, we increase the likelihood of an advective peak emerging in our transit time distribution. Lozier et al. (2013) estimate a transit time distribution for LSW from the Labrador Sea to $25^{\circ} \mathrm{N}$ with a broad peak centered around 25 years, which is a slower spreading rate than inferred in this work. Zou and Lozier (2016) use the same model to examine the export of floats from deep convection areas in the central Labrador Sea. They find a mean age of floats that reach the subtropical boundary (close to Line $\mathrm{W}$ ) of $22 \pm 10$ years, which is also much longer than the transit times we estimate. Jackson et al. (2016) estimate transit time distributions of LSW from the Labrador Sea to $26.5^{\circ} \mathrm{N}$ in the GloSea5 reanalysis product and find a peak at 7 years, which is closer to the time scales found in this work.

It is inherently challenging to compare our results with those from Lagrangian model studies because of the differences in approach. While Lagrangian model studies tend to focus 
on the spreading of particles from one point upstream, and will expose any deviations from a straightforward path along the boundary, our focus on measurements along the boundary will highlight any connectivity that is present. Further, water mass properties represent an integrated result. Individual particles that encounter stirring along the boundary will likely be drawn off the boundary, but the water mass as a whole may persist with modified properties. The only stirring that is present in our estimate has occurred to water parcels that have remained or re-entered the boundary current.

We present evidence for an advective pathway for dLSW along the DWBC from the central Labrador Sea to the subtropical gyre boundary at Line W with a mean transit time of 5 years. As discussed, this is notably shorter than most modeled transit times. In the winter of 2014/2015 there was renewed deep convection in the central Labrador Sea (personal communication, Igor Yashayaev). This offers a rare opportunity in oceanography to conduct a real world experiment by awaiting its arrival at Line $\mathrm{W}$ and obtaining an independent measure of this transit time, potentially clarifying model and observation disagreements. 
Chapter 4

\begin{abstract}
A Model of the Interaction Between the Gulf Stream Northern Recirculation Gyre AND the DeEP Western Boundary CURRENT
\end{abstract}




\subsection{Abstract}

An idealized two-layer quasi-geostrophic model is used to investigate the adjustment of a recirculation gyre forced by an unstable jet to a bathymetric slope at depth. The recirculation gyre is constrained meridionally by the bathymetric slope when the slope is closer to the jet axis than the natural width of the recirculation gyre. We present an analytical prediction of the meridional extent of the gyre, and find that the recirculation gyre can homogenize PV into the slope. This adjustment process leads to an increase in eddy PV fluxes at the base of the slope, which can impact a DWBC traveling on the slope by stirring its water properties with those of the NRG. This effect becomes more pronounced when the instability of the jet is increased. Through variation of the distance between the jet and the slope, we find that proximity to the slope can damp eddy PV fluxes from the unstable jet, and lengthen the recirculation gyre in the along-isobath direction. These mechanisms may shape the circulation in the western North Atlantic, with potential feedbacks on the climate system. 


\subsection{Introduction}

In the western North Atlantic, the Gulf Stream brings warm, salty water of subtropical origin towards the high-latitudes, while the deep-reaching Deep Western Boundary Current (DWBC) brings cold, fresh water of high-latitude origin equatorward. The combined effect of these two currents is a poleward heat transport in the North Atlantic, aiding in the stabilization of the earth's climate (Wunsch, 2005). The Gulf Stream and DWBC come in close contact at Cape Hatteras, where the DWBC is forced under the Gulf Stream (Pickart and Smethie, 1993), and at the Tail of the Grand Banks, where the currents are adjacent (Mertens et al., 2014). The interaction between the Gulf Stream and DWBC at these two locations has been the focus of previous work (e.g. Hogg and Stommel, 1985; Spall, 1996a; Gary et al., 2011; Buckley and Marshall, 2015).

Between Cape Hatteras and the Tail of the Grand Banks, the Gulf Stream has a cyclonic recirculation cell to its north: the Northern Recirculation Gyre (NRG), shown schematically in Figure 4-1. The barotropic NRG coexists with the DWBC at depth (Worthington, 1976; Hogg, 1983), and there is evidence of tracer exchange between them (Hogg et al., 1986; Bower et al., 2009). In this study, we focus on the interaction between the NRG and the DWBC, which is of fundamental interest because of its potential feedbacks on the structure of the overturning circulation in the western North Atlantic and its associated poleward heat transport.

Large-scale ocean dynamics are often cast in terms of Potential Vorticity (PV), a dynamical tracer which is conserved barring forcing or dissipation. In a steady-state balance of PV, eddy motions on short time scales can drive flows across time mean PV contours (Holland and Rhines, 1980). Rhines and Holland (1979) explained that eddies tend to flux PV down-gradient and Rhines and Young (1982) further showed that eddies homogenize PV within closed circulation contours. Through connections to these theoretical developments, Hogg (1983) proposed that the NRG, as well as its anticyclonic counterpart to the south of the Gulf Stream, are driven by Gulf Stream eddies. PV homogenization in the NRG has been reported in climatological observations by McDowell et al. (1982), Bower et al. (1985) and Lozier (1997).

The theory of recirculation gyres developed in several directions, with some studies emphasizing that recirculation gyres can arise from the time-mean inertial terms alone (Fofonoff, 1954; Greatbatch, 1987) and others emphasizing eddy-mean and eddy-eddy interaction (Malanotte-Rizzoli et al., 1995; Berloff et al., 2007). Recent work has emphasized the role of both effects working in tandem (Jayne et al., 1996; Mizuta, 2009); eddies generated by an unstable jet act to smooth potential vorticity, creating homogeneous regions in which inertial recirculations can develop. Waterman and Jayne (2011) studied this phenomenon in a two-layer quasi-geostrophic model, emphasizing the along-stream dependence of eddy 


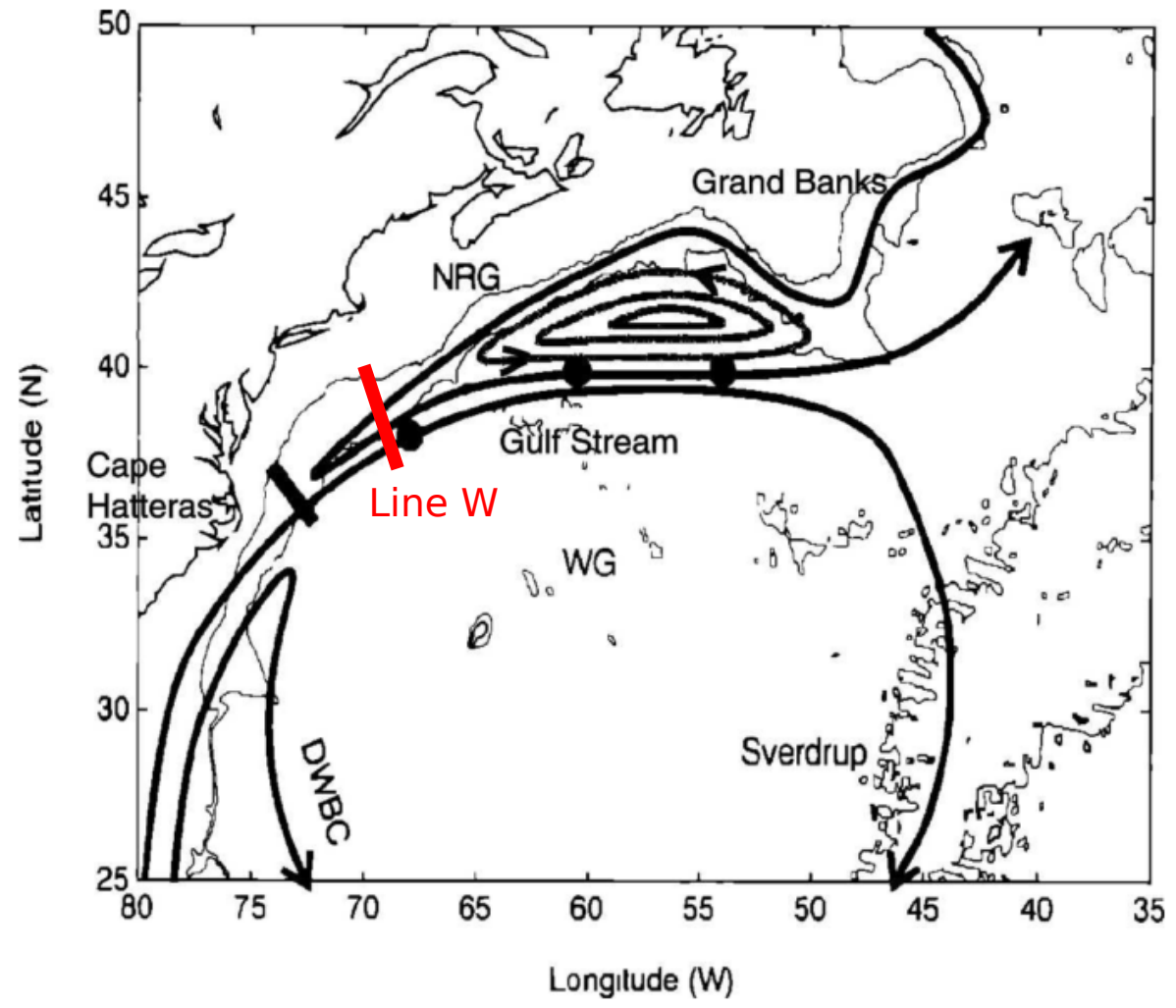

Figure 4-1: Schematic of the barotropic circulation in the western North Atlantic from Zhang and Vallis (2007), adapted from Hogg (1992). The Northern Recirculation Gyre (NRG) is bordered by the Grand Banks and Cape Hatteras to its northeast and southwest respectively, the DWBC to its northwest and the Gulf Stream to its southeast. The approximate position of the Line W moored array is depicted in red.

roles and highlighting that downstream enstrophy convergence results in up-gradient PV fluxes that drive recirculation gyres.

Here, we extend the model of Waterman and Jayne (2011) to include a continental slope to the north of the NRG, as well as a Deep Western Boundary Current (DWBC) on the slope. We investigate how the slope and DWBC affect NRG dynamics and how the NRG influences the DWBC.

Some early work on the NRG emphasized the role of bathymetry in creating closed PV contours for the deep ocean (Hogg and Stommel, 1985; Malanotte-Rizzoli et al., 1995). More recently, Zhang and Vallis (2007) stressed the role of the interaction between the Gulf Stream and the DWBC at both the Grand Banks and at Cape Hatteras in determining the extent of the NRG. In fact, many studies suggest that the DWBC impacts the position and strength of the Gulf Stream and NRG (Hogg and Stommel, 1985; Thompson and Schmitz, 1989; Spall, 1996b; Jackson et al., 2016). The interaction of the DWBC and the NRG between the Grand Banks and Cape Hatteras, however, has not been a focus of the literature.

Observations of the DWBC indicate that spreading rates measured using anthropogenic 
tracers and Lagrangian floats (Pickart et al., 1989; Smethie, 1993; Doney and Jenkins, 1994; Bower and Hunt, 2000) are several times slower than mean DWBC velocities in the western North Atlantic (Schott et al., 2006; Toole et al., 2011), indicating off-slope stirring or recirculation. The structure of this stirring at NRG latitudes was mapped by Hogg et al. (1986) and Pickart and Smethie (1993), who found tongue-like intrusions of salinity, oxygen, and CFC F-11 just north of Cape Hatteras. However, floats released in the DWBC between the Grand Banks and Cape Hatteras followed the DWBC until the crossover point at Cape Hatteras (Bower and Hunt, 2000).

More recent observations focus on the interaction between the DWBC and Gulf Stream extension at the Tail of the Grand Banks, where acoustically tracked neutrally-buoyant floats preferentially left the DWBC (Bower et al., 2009, 2011). These float trajectories were reproduced in high-resolution models and a southward interior pathway for water of subpolar origin emerged within the Gulf Stream's recirculation gyres (Gary et al., 2011). Probability maps of float position show a continuous probability distribution of e-floats from the slope to the off-shore edge of the recirculation gyres, indicating exchange between the DWBC and the NRG.

Observations of the NRG indicate that it is constrained by continental slope bathymetry, coexisting with the DWBC at depth (Hogg et al., 1986). Float observations and highresolution models suggest off-slope stirring of the DWBC into an interior pathway within the NRG; however the detailed dynamics of this interaction remain unclear (Gary et al., 2011). In this work, we use an idealized model setup in order to isolate the dynamics of this interaction, with the goal to inform the interpretation of future observations and realistic modeling efforts.

We first describe the model setup in Section 4.3, followed by a summary of the dynamics of the model jet in Section 4.4. In Sections 4.5 and 4.6 we report on the incremental addition of a bathymetric slope and DWBC to the model, using a conceptual framework for the meridional extent of recirculation gyres and PV budgets as our primary tools. We expand this analysis to an array of jet configurations in Section 4.7, and in Section 4.8, we discuss the implications of this study.

\subsection{Model setup}

Our model is based on the quasi-geostrophic setup introduced in Jayne et al. (1996) to study the dynamics of recirculation gyres. This setup was extended to the present twolayer configuration by Waterman and Jayne (2011). The governing equations for this twolayer quasi-geostrophic model are conservation equations for non-dimensional QGPV (Quasi- 
Geostrophic Potential Vorticity), $q_{n}$, which is given by

$$
q_{n}=\nabla^{2} \psi_{n}+\beta y+(-1)^{n} \frac{1}{S_{n}}\left(\psi_{1}-\psi_{2}\right)+\eta_{B}(n-1)
$$

The subscript $n$ denotes the layer, i.e. $n=1$ corresponds to the upper, surface layer and $n=2$ to the lower, deep layer. $\psi_{n}$ is the non-dimensional streamfunction in the $n$th layer. The QGPV in each layer is the sum of the relative vorticity in the layer, $\zeta_{n}=$ $\nabla^{2} \psi_{n}$; the planetary vorticity $\beta y$, where $\beta$ is a constant approximation to the slope of the Coriolis parameter, $f$; a thickness component proportional to the difference between layer streamfunctions, $(-1)^{n} \frac{1}{S_{n}}\left(\psi_{1}-\psi_{2}\right)$; and a contribution from bathymetry in the lower layer, $\eta_{B} . S_{n}$ is the $n$th layer Burger number,

$$
S_{n}=\left(\frac{N H_{n}}{f_{0} L}\right)^{2}=\frac{g^{\prime} H_{n}}{f_{0}^{2} L^{2}}=\left(\frac{L_{R O}}{L}\right)^{2},
$$

where $H_{n}$ is the layer depth, $f_{0}$ is the scale of the Coriolis parameter (about which $f$ is linearized), $N$ is the buoyancy frequency and $g^{\prime}$ is the reduced gravity, $g^{\prime}=g\left(\frac{\rho_{2}-\rho_{1}}{\rho_{0}}\right)=$ $N^{2} H_{n}$, where $g$ is gravitational acceleration, $\rho_{n}$ is the equivalent constant density of the $n$th layer, and $\rho_{0}$ is a reference density. The reduced gravity is the relevant indicator of stratification in a two-layer system. The Burger number is the squared ratio of the Rossby radius of deformation, $L_{R O}$ and the length scale of motion, $L$, in each layer; it can be thought of as setting the relative depths of the two layers.

The QGPV conservation equations for the upper and lower layers are

$$
\frac{\partial q_{n}}{\partial t}+J\left(\psi_{n}, q_{n}\right)-A \nabla^{4} \psi_{n}+\nabla \cdot\left(R_{n} \nabla \psi_{n}\right)=0
$$

where $J$ is the Jacobian operator, $J\left(\psi_{n}, q_{n}\right)=\frac{\partial \psi_{n}}{\partial x} \frac{\partial q_{n}}{\partial y}-\frac{\partial \psi_{n}}{\partial y} \frac{\partial q_{n}}{\partial x}=u_{n} \cdot \nabla q_{n}, A$ is Laplacian viscosity acting on the relative vorticity to suppress grid-scale noise, and $R_{n}$ is a linear friction coefficient. $R_{2}$ has a small background value for numerical stability throughout the domain and is large in the high-friction sponge layers (see below and Appendix for sponge layer details). $R_{1}$ is only non-zero in the high-friction sponge layer, where it has the same sponge friction value as $R_{2}$.

Non-dimensionalization of the model variables requires a choice of scales. Similar to Waterman and Jayne (2011) and Jayne et al. (1996), we choose the following scales:

$$
\begin{gathered}
L=40 \mathrm{~km} \\
U=0.64 \mathrm{~m} \mathrm{~s}^{-1} \\
H_{1}=800 \mathrm{~m} \\
f_{0}=1 \times 10^{-4} \mathrm{~s}^{-1} .
\end{gathered}
$$

These scales set the relationship between the non dimensional and dimensional values of the 


\begin{tabular}{|l|l|l|}
\hline Dimensionalizing equation & Nondimensional value $(\mathrm{s})$ & Corresponding dimensional value \\
\hline$q_{\text {dim }}=q U / L\left(s^{-1}\right)$ & $q=1$ & $q_{\text {dim }}=1.6 \times 10^{-5} \mathrm{~s}^{-1}$ \\
$\psi_{\text {dim }}=\psi U L\left(\mathrm{~m}^{2} \mathrm{~s}^{-1}\right)$ & $\psi=1$ & $\psi_{\text {dim }}=2.6 \times 10^{4} \mathrm{~m}^{s} \mathrm{~s}^{-1}$ \\
$t_{\text {dim }}=t L / U$ & $t=1$ & $t_{\text {dim }}=17$ hours \\
\hline$\beta_{\text {dim }}=\beta U / L^{2}(m s)^{-1}$ & $\beta=0.05$ & $\beta_{\text {dim }}=2 \times 10^{-11}(\mathrm{~ms})^{-1}$ \\
$R_{\text {dim }}=R U / L\left(s^{-1}\right)$ & $R_{\text {interior }}=10^{-6}$ & $R_{\text {interior dim }}=2000(\text { years })^{-1}$ \\
& $R_{\text {sponge }}(\max )=0.1$ & $R_{\text {sponge dim }}=7(\text { hours })^{-1}$ \\
$A_{\text {dim }}=A U L\left(m^{2} s^{-1}\right)$ & $A=3 \times 10^{-5}$ & $A_{\text {dim }}=1.3 \mathrm{~m}^{2} \mathrm{~s}^{-1}$ \\
$H_{2}=S_{2} H_{1} / S_{1}(m)$ & $S_{1}=1.0, S_{2}=4.0$ & $H_{2}=3200 \mathrm{~m}$ \\
$\eta_{B \operatorname{dim}}=\eta_{B} U H_{2} / f_{0} / L(m)$ & $\eta_{B}=4.0$ & $\eta_{B \text { dim }}=2050 \mathrm{~m}$ \\
\hline
\end{tabular}

Table 4-1: Dimensionalization of variables. Representative scales for variables $q, \psi$ and $t=1$ are shown as well as the fixed value of model parameters.

model variables, as reported in Table 4-1.

The model is forced by the prescribed flow at its boundaries. At the western, eastern and southern boundaries of the model domain, sponge layers with very high friction minimize the effect of the prescribed in- and outflows on the interior dynamics. Note, however, that the sponge layer at the western boundary does play a dynamically important role in closing the recirculation gyre momentum and PV budgets. The western sponge is $400 \mathrm{~km}$ wide, and the eastern and southern sponge layers are $800 \mathrm{~km}$ wide. At the northern boundary, there is a wall with a no normal flow and free-slip boundary condition that mimics the continental boundary.

The model domain is $5,000 \mathrm{~km}$ in the north-south direction and $16,000 \mathrm{~km}$ from west to east, in order to isolate the interior dynamics from the eastern boundary outflow condition and accommodate the zonal lengthening of the recirculation gyres when a slope is added. The model resolution is $4 \mathrm{~km}$, so that the minimum Rossby radius of deformation in the domain, $40 \mathrm{~km}$, is well-resolved. Figures will only display the western third and northern quarter of the model domain, often excluding the western sponge layer. The full model domain and sponge configuration are discussed further in the Appendix and illustrated in Figure 4-24.

In the upper layer, an unstable jet enters the rectangular model domain in the west and exits in the east. The inflowing zonal velocity structure, specified at the western boundary of the domain, is Gaussian in latitude. The PV associated with the jet changes sign in both the vertical and horizontal, as depicted in Figure 4-2 for the basic model setup, rendering it unstable to mixed barotropic, baroclinic instability. The meridional structure of PV in the upper layer is dominated by the large PV gradient associated with the jet. In this study, the jet represents the Gulf Stream extension, which has a primarily zonal orientation. As in Waterman and Jayne (2011), the outflow condition specified at the eastern boundary of the 

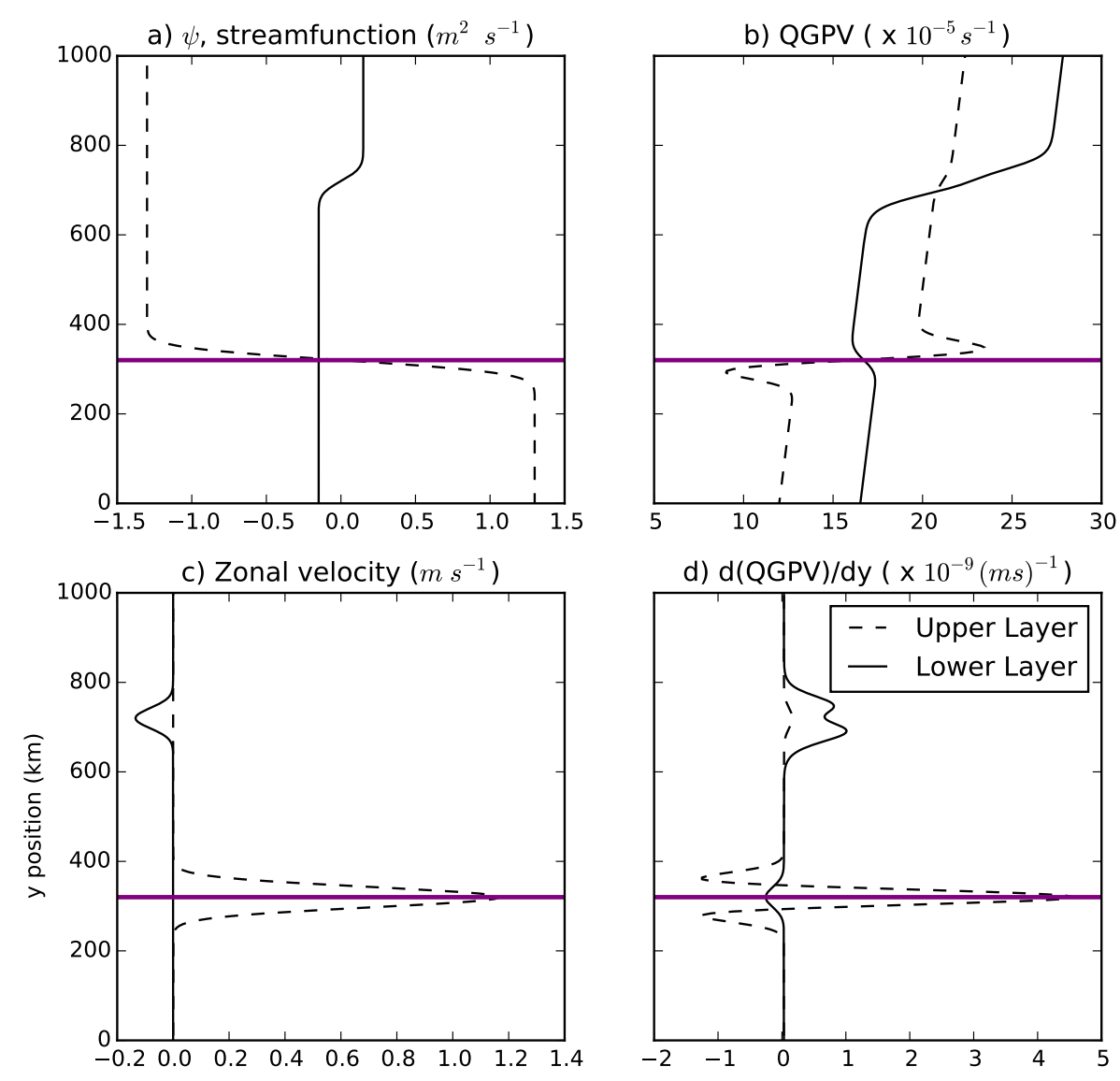

Figure 4-2: Meridional structure of the a) streamfunction, b) potential vorticity c) velocity, and d) meridional potential vorticity gradient at the model western boundary. Upper layer properties are dashed and lower layer properties are solid. A strong Gulf Stream jet flows eastward in the upper layer, and is unstable to mixed barotropic baroclinic instability as its associated PV gradients change sign in the horizontal and vertical. In the lower layer, a DWBC flows in the opposite direction. The DWBC is stabilized by a bathymetric gradient in the lower layer. The horizontal purple line denotes the meridional position of the jet, and will be used throughout as a reference point.

domain is a marginally stable jet profile. This difference in PV structure from west to east is a critical control on the strength of the recirculation gyre, as was shown in Waterman and Jayne (2011).

To the north of this jet, in the lower model layer, a DWBC flows in the opposite direction: entering the model domain in the east and exiting in the west with the same profile. As shown in Figure 4-2, the DWBC maximum velocity is 9 times smaller than the unstable jet, and travels on a bathymetric slope, which stabilizes the DWBC to barotropic and baroclinic instability. The meridional PV structure of this layer is dominated by the bathymetric slope. 

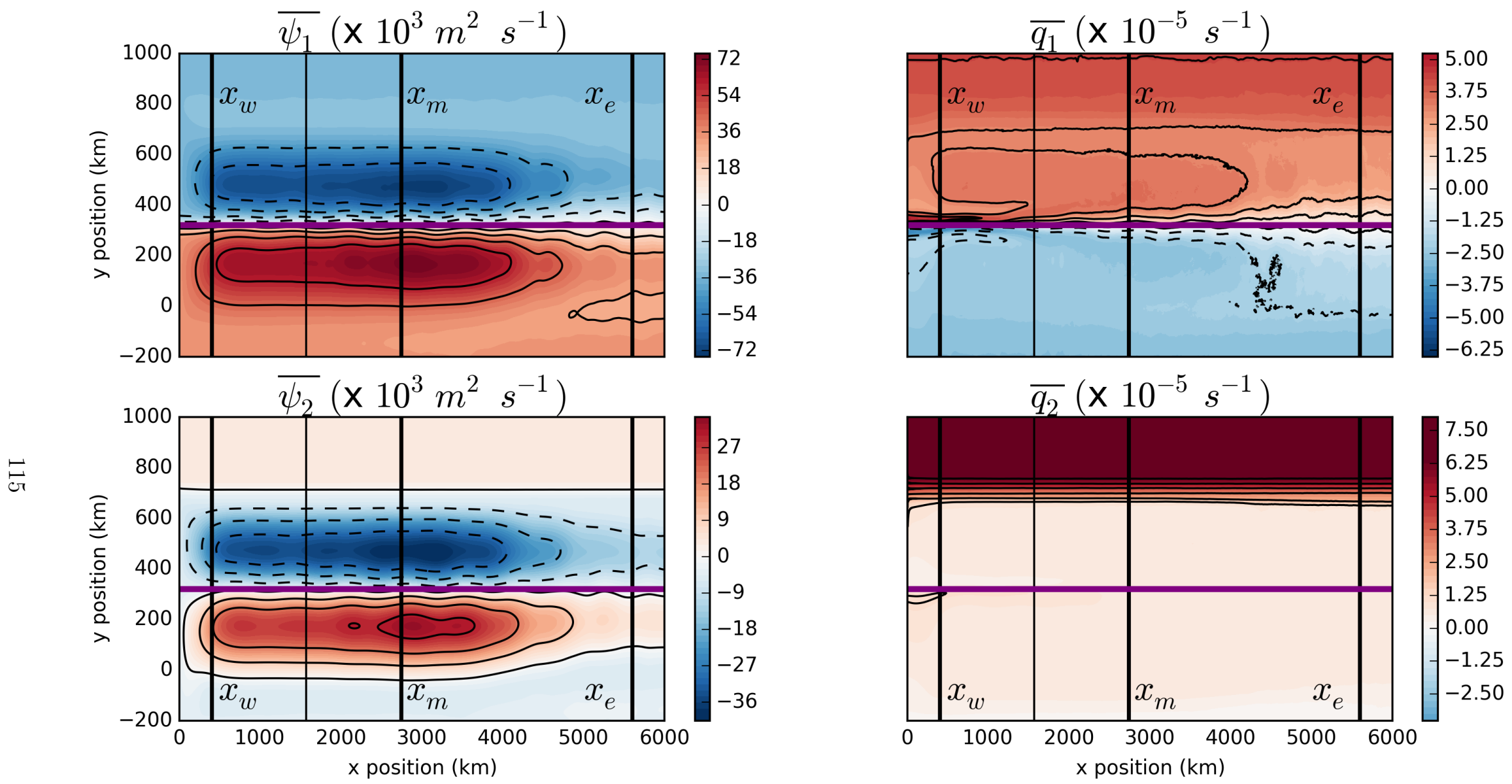

Figure 4-3: Mean streamfunction $(\bar{\psi})$ and PV $(\bar{q})$ in the upper and lower layers for the basic model setup. Thick vertical lines indicate the eastern boundary of the western sponge region, which is the western boundary of our recirculation gyre analyses, $x_{w}$, the zonal position of jet stabilization, $x_{m}$, and the eastern boundary of the recirculation gyre, $x_{e}$. Subsequent figures and analysis will refer to these zonal boundaries. The halfway point between $x_{w}$ and $x_{m}$ is also shown, which separates parts 1 and 2 referenced in Figure 4-5. The horizontal purple line denotes the meridional position of the jet. 
The imposed transport of the upper layer jet is 55 Sverdrups ( $\mathrm{Sv}, 10^{6} \mathrm{~m}^{3} \mathrm{~s}^{-1}$ ). In steady state, recirculation gyres form to the north and south of the jet in the upper layer, as depicted in Figure 4-3, increasing the transport to $120 \mathrm{~Sv}$ at the point of jet stabilization. This is in direct analogy to the increase in Gulf Stream transport from $30 \mathrm{~Sv}$ in the Florida Straits, to $65-95 \mathrm{~Sv}$ at Cape Hatteras, to $150 \mathrm{~Sv}$ by $60^{\circ} \mathrm{W}$ (Knauss, 1969; Worthington, 1976; Leaman et al., 1989; Hogg, 1992; Meinen and Luther, 2016). The speed of the recirculation gyres compares well with observations from Bower and Hogg (1996), in both the model and observations they are on the order of tens of $\mathrm{cm} \mathrm{s}^{-1}$.

Including transport in the lower layer increases the total jet transport by a further 200 Sv, which renders the total jet transport unrealistically large. It may be that the flow field in the deep ocean is too noisy to measure this contribution to the jet transport in the real ocean, but this large transport raises questions about the relevance of our parameter space. As this parameter space was found to be relevant to the Kuroshio (Waterman et al., 2011), and the dynamics of this parameter space is well understood, we proceed with this caveat in mind.

The DWBC is composed of water masses from several subpolar origins. Labrador Sea Waters, formed in the Labrador Sea, occupy the intermediate water depths $(\approx 500-1000 \mathrm{~m})$, and Overflow Waters formed in the Nordic Seas generally lie below a water depth of $2000 \mathrm{~m}$. In this two layer model, we focus only on the deep component of the DWBC, which can be thought of as the Overflow Water component of the DWBC. Spall (1996a) used a three layer model to simulate the DWBC, with the lowest layer representing Overflow Waters and the intermediate layer representing Labrador Sea Waters. This was not possible in this model, as the slope would have to extend into the intermediate layer and there cannot be vanishing layer thicknesses in QG. The two-layer system also keeps the problem tractable. The absence of a Labrador Sea Water-like water mass is a limitation of the model that will be discussed further in Section 4.8.

The DWBC we impose has a transport of $17 \mathrm{~Sv}$ in this lower layer, which is consistent with observations of deep DWBC transports at NRG latitudes (Schott et al., 2006; Toole et al., 2011). We use the structure of the DWBC measured at the Line $\mathrm{W}$ moored array to inform the structure of the model DWBC. Line $\mathrm{W}$ is on the continental slope at $39^{\circ} \mathrm{N}$, at the southern edge of the NRG, as depicted in Figures 4-1 and 4-4. The deep components of the DWBC lie between the 2500 and $4500 \mathrm{~m}$ isobaths at Line $\mathrm{W}$, in bottom intensified cores with mean maximum equatorward velocities of about $8 \mathrm{~cm} \mathrm{~s}^{-1}$. The model DWBC similarly has a maximum speed of $10 \mathrm{~cm} \mathrm{~s}^{-1}$ and lies between the 2000 and $4000 \mathrm{~m}$ isobaths.

The DWBC flows equatorward along the continental slope in the western North Atlantic. As shown in Figure 4-4, the seafloor deepens dramatically from the shelf break to $2000 \mathrm{~m}$ water depth and then more gradually until it reaches 4000m depth. The deep DWBC flows on this more gradual incline at depth, and we simulate this component of the continental 
slope in our model setup, as the QG approximation limits the steepness of the slopes we can simulate. The model continental slope has an error function profile, so that the steepness of the slope has a Gaussian profile in latitude.

The PV in the lower layer is dominated by a steep meridional gradient due to the bathymetric slope, as shown in Figure 4-3. Within the recirculation gyres to the south of the slope, PV is homogeneous. This is analogous to the PV structure of the deep ocean in the northern recirculation region as mapped by Talley and McCartney (1982), Bower et al. (1985) and Hogg et al. (1986). This analogy motivates the zonal model setup: the mean planetary PV contours are overcome by the strong bathymetry gradient and PV homogenization. However, in reality, additional effects are likely present due to the southward component of the DWBC and northward tilt of the Gulf Stream extension.

A drawback of the present model is that it manifests standing meanders at the jet axis, which are likely resonant Rossby wave modes. These standing waves are suppressed by the high friction sponge layers, but are still apparent, especially when there is weaker mean flow (see Figure 4-13). They do not appear to play an important role in our analysis, but they are an unrealistic component of the model.

We use a range of model configurations to gain understanding of the system dynamics. We first focus on a set of three model configurations to isolate the effects of a slope and DWBC in the lower layer. The first of these three configurations does not include a slope or DWBC in the lower layer, the second includes a slope $200 \mathrm{~km}$ wide and $2000 \mathrm{~m}$ high in the lower layer, with its center $400 \mathrm{~km}$ from the center of the jet, and the third, which is depicted in Figure 4-2, includes a DWBC on the slope.

We then vary the instability of the jet and the distance between the jet and the slope. These parameter variation runs include a slope and DWBC which have the same initial parameters as the basic setup. Our focus is on jet parameters because the DWBC is a largely passive component of the model configuration. Our interest in the distance between the jet and the slope is inspired by observations that the NRG is constrained by bathymetry, as displayed in Figure 4-1.

All model configurations are run for a total of 6,500 non-dimensional time units, which corresponds to 13 physical years. Our focus will be on averages and statistics between model years 3 and 8. Model configurations with a slope in the lower layer did not achieve statistical steady state over this time, as we will discuss further. The model development beyond model year 8 is clouded by unrealistic influences from the southern sponge. This issue is explained in the Appendix along with additional technical model details. 

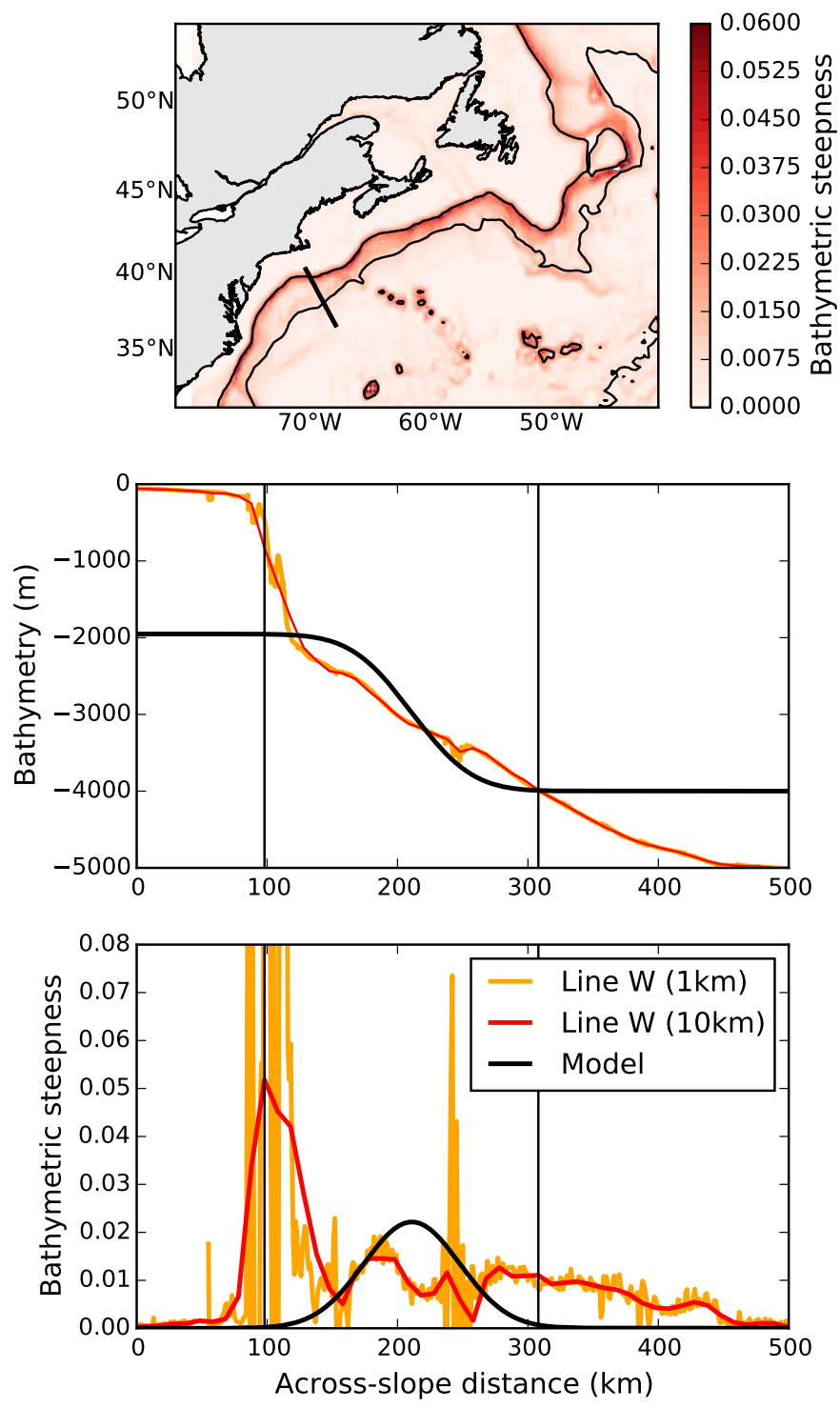

Figure 4-4: Steepness of bathymetry along the continental slope, and comparison of Line W and model bathymetry and steepness. a) Map of bathymetric steepness along continental slope. $1000 \mathrm{~m}$ and 4000m isobaths, as well as Line $\mathrm{W}$ cruise track are shown in black. b) Comparison of bathymetry along Line $\mathrm{W}$ cruise track with $1 \mathrm{~km}$ and $10 \mathrm{~km}$ resolution (in red and orange respectively) and in the primary model setup (in black). Distance is measured from the continental shelf for the Line $\mathrm{W}$ track, and the northern boundary in the model setup. Vertical black lines are $1000 \mathrm{~m}$ and $4000 \mathrm{~m}$ isobath locations on Line $\mathrm{W}$ track for reference. c) Comparison of bathymetric steepness along Line $\mathrm{W}$ cruise track (in red and orange) and in the primary model setup (in black). Distance and vertical lines as in b). 


\subsection{Jet evolution}

Waterman and Jayne (2011) describe the eddy-mean flow interactions that drive the inertial recirculation gyres in this idealized setup. They emphasize that the nature of the eddy effects depends critically on the along-stream position. As explained in Rhines and Holland (1979), the eddy flux of PV tends to be down the mean PV gradient. This emerges from the steady state equation for the conservation of enstrophy, the square of eddy PV. The down-gradient PV flux is a consequence of the dissipation of velocity and density necessary to achieve steady state in the enstrophy balance. However, if there is a significant local convergence of enstrophy, through eddy decay for example, eddies can flux PV up the mean gradient.

As the unstable jet enters the domain, it is stabilized by a down-gradient eddy PV flux. Downstream, where the jet has been stabilized, an eddy enstrophy convergence allows up-gradient eddy PV fluxes, which drive the recirculation gyres through nonlinear eddy rectification. This convergence of eddy enstrophy arises because of eddy decay: eddies are produced upstream in the unstable jet and grow and decay as they are advected by the jet. Once the jet is stabilized by these eddies, it no longer produces eddies, and it is this asymmetry that causes a convergence of enstrophy. PV is homogenized by the eddies in the recirculation gyres, so that inertial flow can also develop within them.

In the two layer case, which is the focus of this work, the same mechanism is at play, with some additional complications. In two layers, the jet must be stabilized with respect to both its horizontal and vertical shear. In this section, we describe the evolution of the jet in the basic model setup described in Section 4.3, which includes a slope in the lower layer and a DWBC. The addition of the slope and DWBC do not alter the jet evolution significantly in this model configuration, but as we describe in Section 4.7, this is not always the case.

In the western section of the gyre, the horizontal shear of the jet is dominant, though there are changes in the sign of the PV gradients in both the horizontal and vertical directions in both layers, as shown in the left panels of Figure 4-5. The jet is stabilized with respect to its horizontal shear first, by strong down-gradient eddy PV fluxes in the upper layer. Fluxes in the lower layer are also down-gradient, but increase along the jet axis, as displayed in the center panels of Figure 4-5. This occurs because variability has been transferred from the upper layer to the lower layer through thickness fluxes and the vertical shear becomes dominant over the horizontal shear.

The maximum recirculation occurs at the point when the jet has been stabilized to both its horizontal and vertical shear. Beyond the point of maximum recirculation the eddy fluxes in the upper layer are up-gradient, driving the recirculation gyres as in the barotropic case. As shown in the right panels of Figure 4-5, these fluxes are smaller than the previous stabilizing fluxes and are a residual of the eddy relative vorticity fluxes and eddy thickness 
a) West of recirculation max
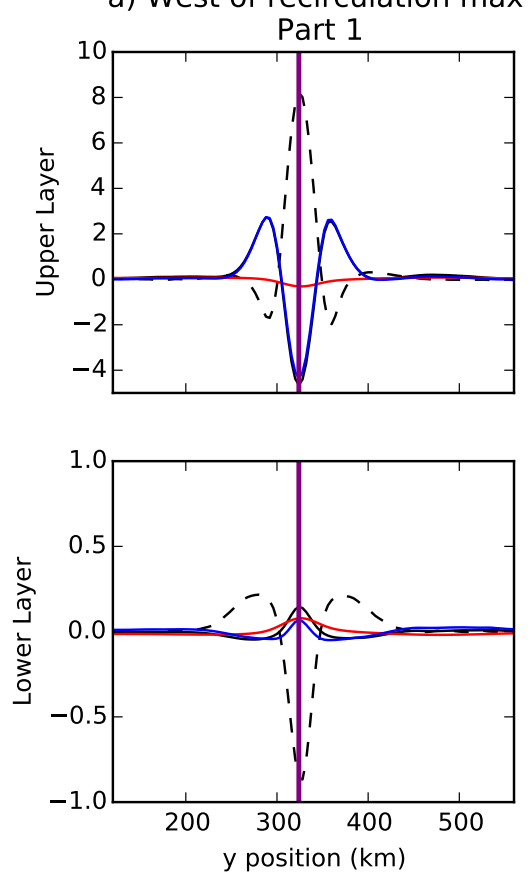

b) West of recirculation max Part 2
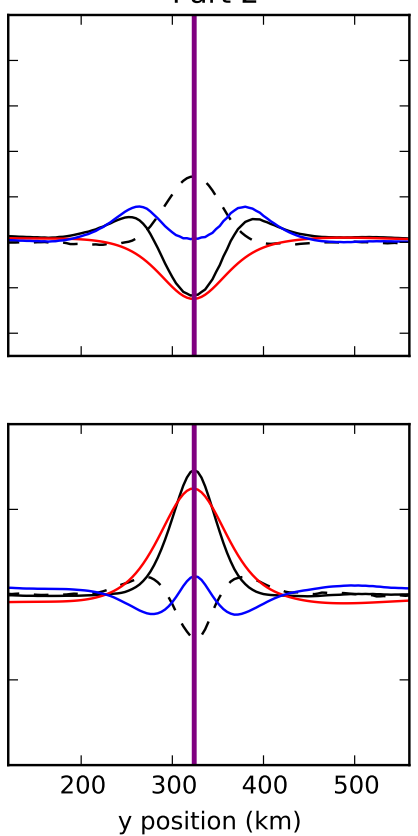

c) East of recirculation max
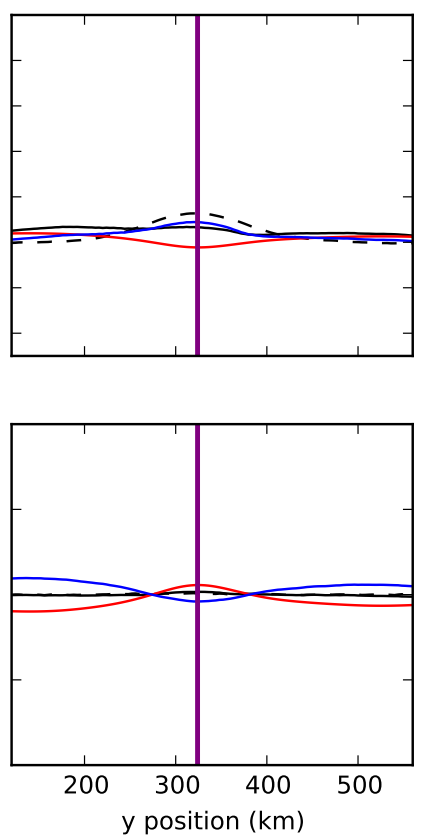

\begin{tabular}{|llll|}
\hline-- & meridional PV gradient & - & meridional eddy thickness flux \\
- & total meridional eddy PV flux & - & meridional eddy relative vorticity flux \\
\hline
\end{tabular}

Figure 4-5: Normalized meridional PV gradients and meridional eddy PV fluxes for averages in sections of the recirculation gyre depicted in Figure 4-3. a) Average between $x_{w}$ and $\left(x_{m}+x_{w}\right) / 2$. b) Average between $\left(x_{m}+x_{w}\right) / 2$ and $x_{m}$. c) Average between $x_{m}$ and $x_{e}$. Top row are upper layer properties, and bottom row are lower layer properties. PV gradients and eddy fluxes are normalized such that quantities of each can be compared between panels. Note the difference in scale between the upper and lower layer panels. Vertical purple lines denote the meridional position of the jet.

fluxes. As discussed in Waterman and Jayne (2011), the eddy relative vorticity fluxes in the upper layer act in the same sense as in the barotropic case. The eddy thickness fluxes act to make the system more barotropic: in the upper layer the thickness fluxes act against the relative vorticity fluxes to slow the stronger recirculation gyres and in the lower layer the thickness fluxes accelerate the weaker recirculation gyres.

\subsection{The meridional extent of recirculation gyres}

Jayne et al. (1996) and Jayne and Hogg (1999) constructed a conceptual model of the meridional distribution of PV in their barotropic QG model, the precursor to the model in this study. In the barotropic case, eddies stabilize the jet and form terraces of homogeneous PV to its north and south. This is the case in the upper layer of the two layer model as well. They use this known basic form, in conjunction with the conservation of mass and the assumption that the recirculation gyres are encompassed within the terraces of 
homogeneous PV, to predict the level and extent of the homogeneous recirculation gyre. Their model also relies on the ad hoc assumption that the meridionally integrated vorticity anomaly is conserved.

We can use a similar principle to derive an analytic solution for the PV distribution in the lower layer of the present model. The initial meridional structure of PV in the lower layer is a superposition of planetary beta and a vortex stretching component due to the overlying jet, as shown in Figure 4-6. The gradient of the vortex stretching component is opposite that of planetary beta and the jet is stabilized when this negative PV gradient is eliminated by homogenization.

PV is homogenized about the center of jet, to the average initial PV of the homogenized region. Because the PV beyond the center of the jet increases to the north and decreases to the south, and the eddies homogenize symmetrically about the center of the jet, the final $\mathrm{PV}$ in the homogenized region in the lower layer will be the initial PV at the center of the jet. Applying the assumption that the meridionally integrated PV is conserved, and that the PV structure beyond the homogenized region remains unchanged, we can find the point at which the initial and homogenized PV profiles will intersect, i.e. $y_{r}$, the meridional point at which

$$
\int_{y_{j}}^{y_{r}} q_{i n i t}(y) d y=q_{i n i t}\left(y_{j}\right) \times\left(y_{r}-y_{j}\right),
$$

where $y_{j}$ is the meridional position of the center of the jet and $q_{i n i t}(y)$ is the initial meridional profile of PV in the lower layer.

Note that in this conceptual model, the extent of PV homogenization and the intersection of the initial and steady state profiles are equivalent, and there is a discontinuity in PV at this point. However, in the QG model, there is a region with a finite gradient between the northernmost extent of PV homogenization and the intersection of the initial and homogenized PV profiles, as shown in Figure 4-6. This intermediate region plays an important role in the PV budget of the recirculation gyre and DWBC, as we will discuss in Section 4.6. Because of this difference, $y_{r}$ corresponds to a point between the extent of the region of homogenized PV and the intersection of the initial and final PV profiles.

This analytic expression has some skill in predicting the meridional extent in the QG model when the recirculation gyres do not intersect a slope in the lower layer, as shown in Figure 4-7 for model configurations with a range of inflowing jet velocities (from 0.4 to 1.3 $m s^{-1}$ ). Here, we define the meridional extent of the northern recirculation gyre in the QG model as the average of the extent of the region of homogenized PV and the intersection of the initial and mean PV profile.

As seen in Figure 4-6, the analytic framework also has some skill in predicting the extent of the recirculation gyre when there is a slope in the lower layer. Whether a slope or DWBC 

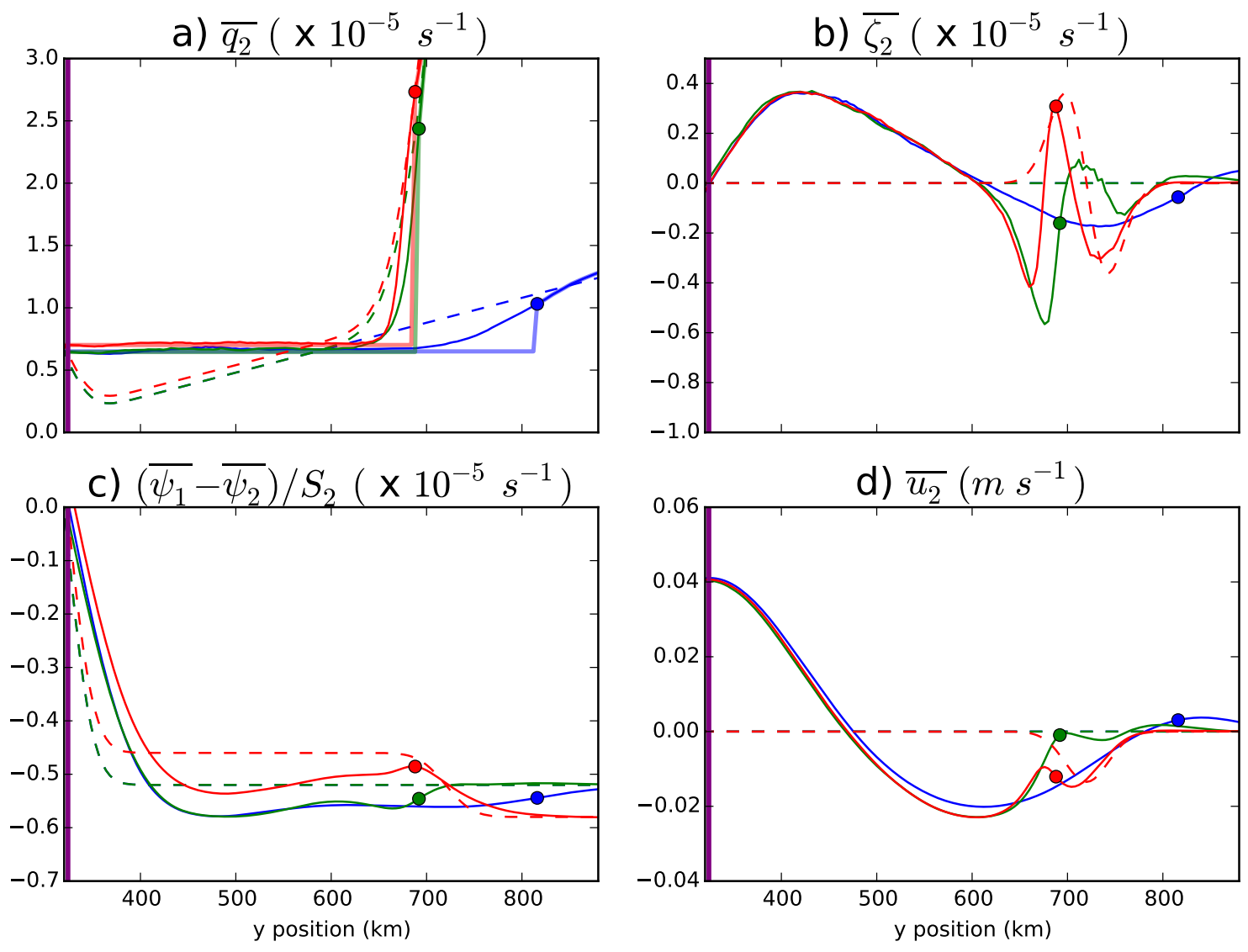

Figure 4-6: Time-mean profiles of lower layer a) $\mathrm{PV}, \overline{q_{2}}, \mathbf{b}$ ) relative vorticity, $\left.\overline{\zeta_{2}}, \mathbf{c}\right)$ thickness, $\left(\overline{\psi_{1}}-\overline{\psi_{2}}\right) / S_{2}$, and $\mathbf{d}$ ) zonal velocity, $\overline{u_{2}}$, in the NRG at the zonal position of jet stabilization for model configurations without a slope or DWBC (blue), with a slope, but no DWBC (green), and with both a slope and DWBC (red). We show properties north of the jet axis, as indicated by the vertical purple line at the left edge of each panel. Dotted lines indicate initial conditions and solid lines the mean in steady state. Lighter colored thick lines in a) are analytic predictions of recirculation gyre extent based on Equation 4.3. Colored points indicate the value of each property at the recirculation gyre boundary, $y_{r}$.

is included in the model, the QG model PV in the homogeneous region at the level of jet stabilization is equivalent to the initial model PV at the jet axis, as assumed in the analytical framework.

In general, bathymetry can constrain the meridional size of the recirculation gyre as it corresponds to a large PV gradient. In terms of Equation 4.3, the LHS reaches the same value as the RHS for a more southerly $y_{r}$ because this large PV gradient increases the LHS quickly. The slope's PV gradient has additional consequences, such as additional eddy PV fluxes at the base of the slope and we will discuss the applicability of this framework further as we present the model PV budgets and parameter variations in the following sections.

As shown in Figure 4-6, the region of homogenized PV is achieved through the relative vorticity of the recirculation gyres, which is counter to the vortex stretching term in the 


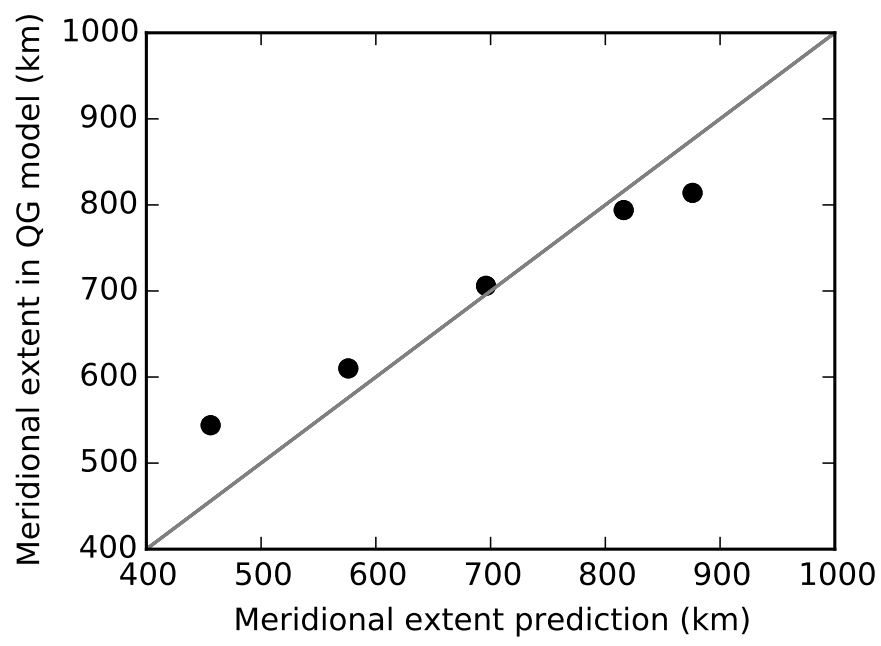

Figure 4-7: Analytic recirculation gyre meridional extent prediction plotted against measured meridional extent in the QG model (both in $\mathrm{km}$ ) for model configurations in which the recirculation gyre does not intersect a slope in the lower layer for model configurations with different inflowing jet velocities $\left(0.36,0.63,0.9,1.17\right.$ and $\left.1.3 \mathrm{~m} \mathrm{~s}^{-1}\right)$. The meridional extent in the QG model is defined as the average of the extent of the region of homogenized PV and the intersection of the initial and mean PV profile. The diagonal grey line is the $\mathrm{y}=\mathrm{x}$ line for reference.

center of the gyre and counter to beta beyond. When there is a bathymetric slope in the lower layer, the zonal return flow is constrained to a more southerly $y_{r}$, which results in a large negative relative vorticity at the base of the slope that homogenizes the PV into the slope.

The increase in the magnitude of velocity in the northern recirculation gyre does not compensate for its shorter meridional extent, and the transport of the recirculation gyre decreases (from 133 to $117 \mathrm{~Sv}$ ). The transport in the southern recirculation gyre stays constant when a slope is added to the model (119 Sv), but there is a consequent decrease in the lower layer jet-like transport (from 253 to $237 \mathrm{~Sv}$ ). The initial difference between the transport in the northern and southern recirculation gyres is due to the layer thickness change across the jet. As noted in Section 4.3, these transports are unrealistically large, but the dynamics of the system are in a relevant parameter space.

When a DWBC is added to the slope, there is positive relative vorticity at the base of the slope which increases the PV gradient at the base of the slope, as well as a vortex stretching contribution which decreases the overall PV gradient. The net effect of adding the DWBC is a decrease in the PV gradient at the slope, with a local increase at the relative vorticity maximum. In the base case shown in Figure 4-6, the recirculation gyre return flow does not have a zero crossing, but merges with the DWBC. Because of this, the relative vorticity minimum is not as large, and homogenization into the slope is inhibited. The redistribution of recirculation gyre transport in the lower layer is the same as that in the case with a slope but no DWBC. 


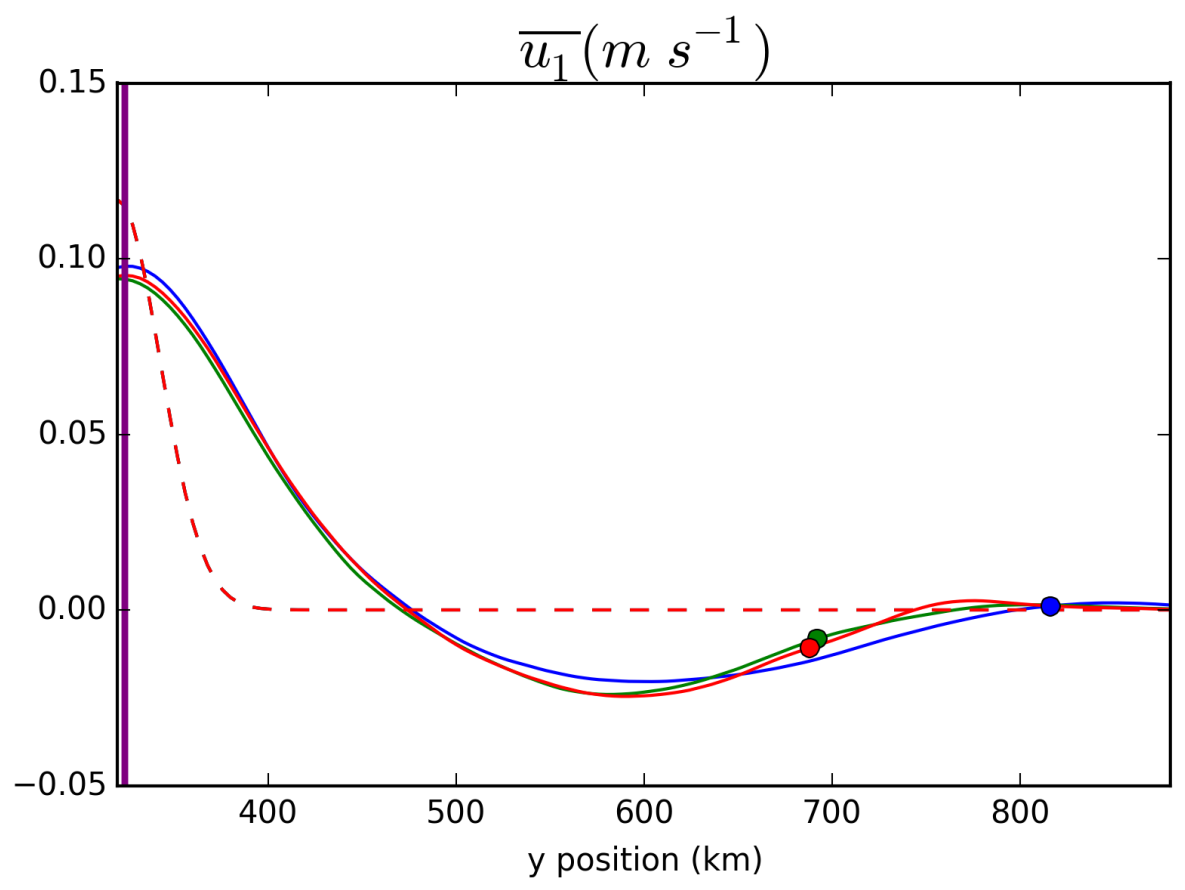

Figure 4-8: Time-mean zonal velocity profiles in the upper model layer at the zonal position of jet stabilization north of the jet axis, as indicated by the vertical purple line at the left edge of the figure. Colored circles denote the position of the predicted extent of the recirculation gyre in the lower layer.

The extent of the recirculation gyre in the upper layer is also affected by the presence of the slope in the lower layer. As shown in Figure 4-8, the meridional extent of the gyre is shortened when there is a bathymetric slope in the lower layer, though the zero crossing is farther north than in the lower layer. The only differences between these model configurations are in the lower layer; thickness fluxes from the lower layer into the upper layer are large enough to change the flow structure in the upper layer.

\subsection{PV budget analysis}

To gain insight into how these mean patterns are maintained as a slope and DWBC are added to the model, we construct PV budgets of the NRG-DWBC system.

The time-averaged steady-state PV equations are

$$
\overline{\frac{\partial q_{n}^{\prime}}{\partial t}}+J\left(\overline{\psi_{n}}, \overline{q_{n}}\right)+\overline{J\left(\psi_{n}^{\prime}, q_{n}^{\prime}\right)}-A \nabla^{4} \overline{\psi_{n}}+\nabla \cdot\left(R_{n} \nabla \overline{\psi_{n}}\right)=0
$$

where overlines indicate time-averaged values and primes are deviations from the time mean. To construct a PV budget for a certain region, we take the integral of these equations over the area of interest. By Gauss' theorem, divergences integrated over an area are equivalent to the contour integral of the fluxes out of the area, so that the integral of these equations 

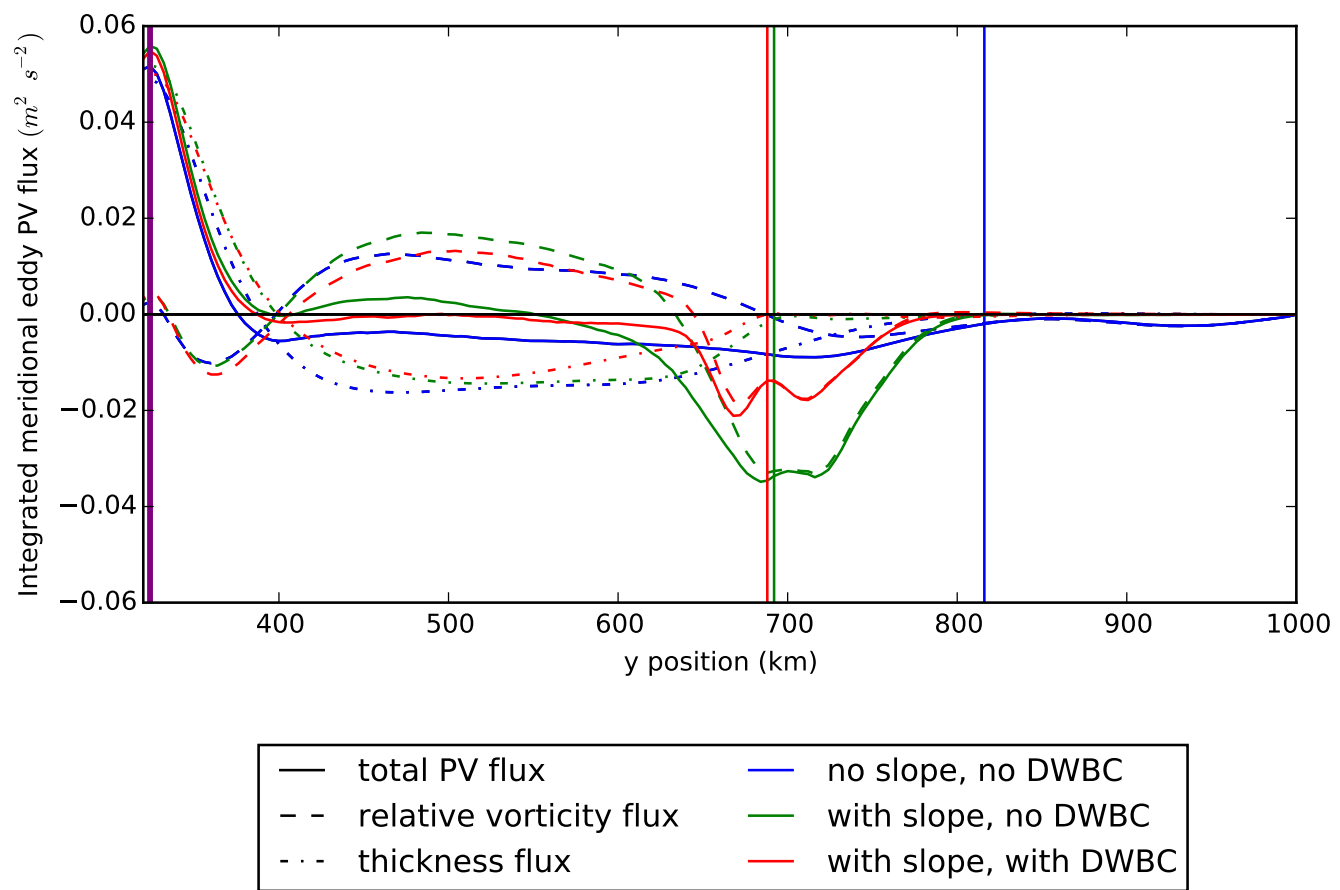

Figure 4-9: Profiles of meridional eddy PV flux in the lower layer integrated zonally across the recirculation gyre in $\mathrm{m}^{2} \mathrm{~s}^{-2}$ for model configurations without a slope or DWBC (blue), with a slope, but no DWBC (green), and both a slope and DWBC (red), i.e. $\int_{x_{w}}^{x_{e}} \overline{v_{2}^{\prime} q_{2}^{\prime}} d x$, where $x_{w}$ and $x_{e}$ are as shown in Figure 4-3. Dashed lines are the contributions from the relative vorticity flux component, $\overline{v_{2}^{\prime} \zeta_{2}^{\prime}}$ and dash-dotted lines are contributions from the thickness component, $\overline{v_{2}^{\prime}\left(\psi_{1}^{\prime}-\psi_{2}^{\prime}\right) / S_{2}}$. Vertical lines indicate the predicted meridional extent of the NRG for each case.

can be written:

$$
\iint \frac{\overline{\partial q_{n}^{\prime}}}{\partial t} d A=-\oint\left(\overline{\mathbf{u}_{\mathbf{n}}} \overline{q_{n}}+\overline{\mathbf{u}_{\mathbf{n}}^{\prime} q_{n}^{\prime}}-A \nabla\left(\nabla^{2} \overline{\psi_{n}}\right)+R_{n} \nabla \overline{\psi_{n}}\right) \cdot \mathbf{d n}
$$

where $\mathbf{u}_{\mathbf{n}}$ is the layer velocity vector, $\mathbf{u}_{\mathbf{n}}=\left(u_{n}, v_{n}\right)=\left(-\frac{\partial \psi_{n}}{\partial y}, \frac{\partial \psi_{n}}{\partial x}\right)$, and $\mathbf{d n}$ is the unit vector normal to the integration contour. The viscosity is a small term in the budget, and the linear drag is only significant in the high friction sponge layers. In the model interior, the primary balance is between the mean and eddy flux divergences of PV. We report fluxes as positive into the budget region, i.e. in the form $-\oint\left(\overline{\mathbf{u}_{\mathbf{n}}} \overline{q_{n}}\right) \cdot \mathbf{d n}$, as in Equation 4.5.

The primary PV balance from the jet center to the northern edge of the recirculation gyre is a balance between a convergence of eddy PV fluxes and a divergence of the mean PV fluxes, or down gradient eddy PV fluxes balanced by an up gradient mean flux of PV. The convergence of eddy PV flux is dominated by meridional eddy PV fluxes. As shown in Figure 4-9, the zonally integrated meridional eddy PV fluxes are positive at the jet center, down the mean negative PV gradient, and negative at the northern extent of the recirculation gyre, down the mean positive PV gradient there, leading to a convergence.

First, consider the PV balance for the NRG in a model configuration without a slope. 


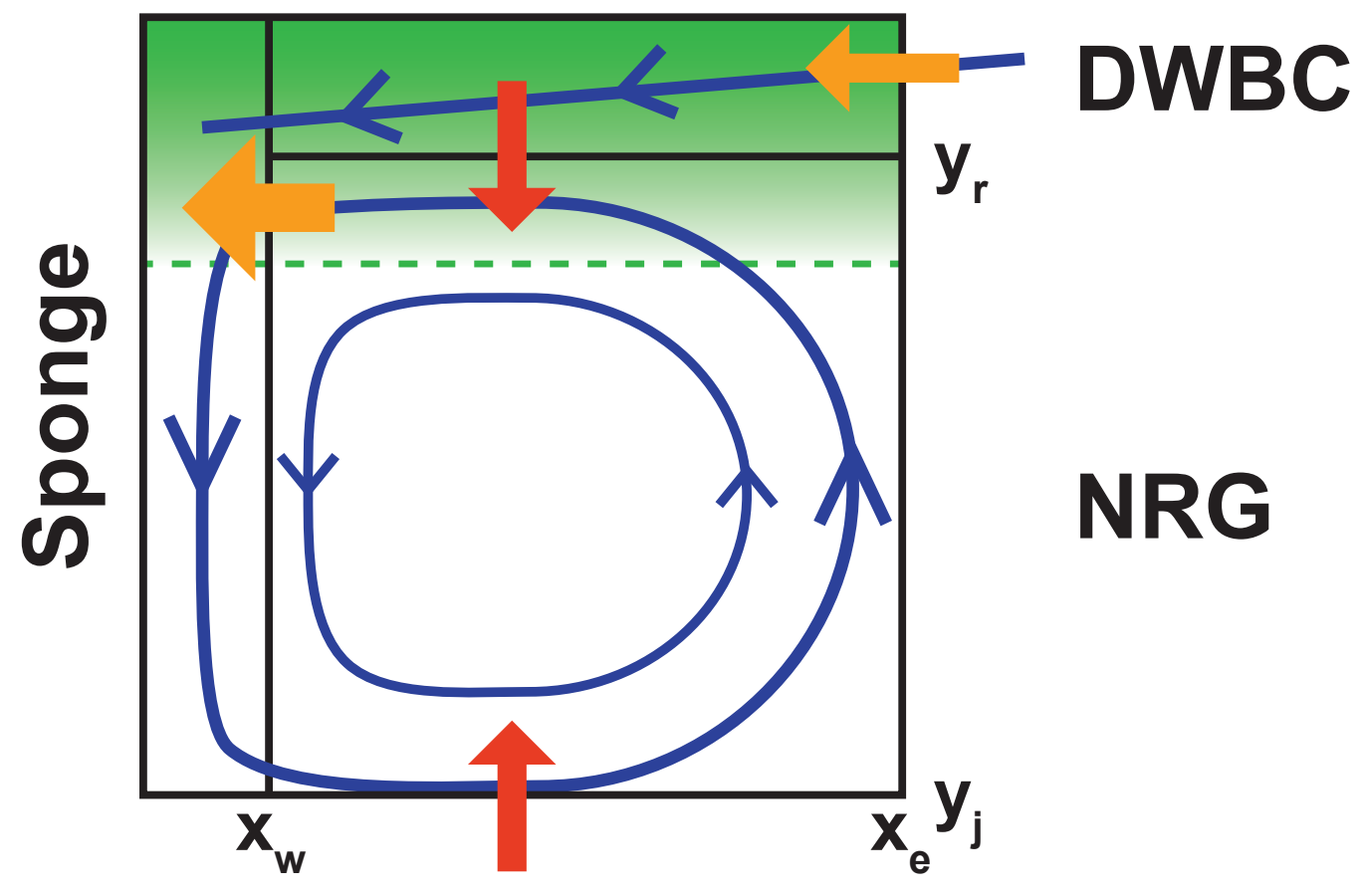

Figure 4-10: Schematic illustration of the system PV budget in the model lower layer. Black lines denote the boundaries between the NRG, DWBC and sponge regions, and green shading represent the PV gradient north of the recirculation gyre, with the dotted green line highlighting the beginning of a non-zero PV gradient. Blue lines are schematic streamlines. Red arrows represent the mean eddy PV fluxes across the boundaries, and orange arrows represent the sense of the mean PV fluxes. The mean PV flux divergence in the NRG region is a result of the outermost recirculation gyre streamline which crosses into higher PV. This streamline is returned to the jet axis in the high friction sponge region. The eddy PV flux divergence out of the DWBC region sustains a mean PV flux convergence: the DWBC enters the recirculation gyre region at higher PV than it exits with, as it flows from a PV gradient dominated by the bathymetric slope to lower PV as a result of homogenization at recirculation gyre longitudes.

The unstable jet sheds eddies that homogenize PV from the jet axis in the lower layer. These eddies first homogenize the meridional PV profile to the jet's negative PV gradient and then to the positive planetary PV gradient to the north of the jet. As highlighted in Section 4.5, there is a PV gradient within the recirculation gyre, between the region of homogenized PV and the intersection of the initial and steady state PV profiles. The PV gradient in this region becomes ever steeper as PV is homogenized farther north, creating an ever-larger barrier to the homogenizing eddies, which flux PV less effectively as they get farther from the jet. The northern edge of the westward return flow of the recirculation gyre falls within this region, and this corresponds to a mean up-gradient PV flux, since these water parcels originate from the homogenized region with lower PV. This mean up-gradient PV flux is compensated by the down-gradient eddy PV fluxes at the northern edge, which supply high PV from the north. In summary, the eddy PV flux convergence due to the 

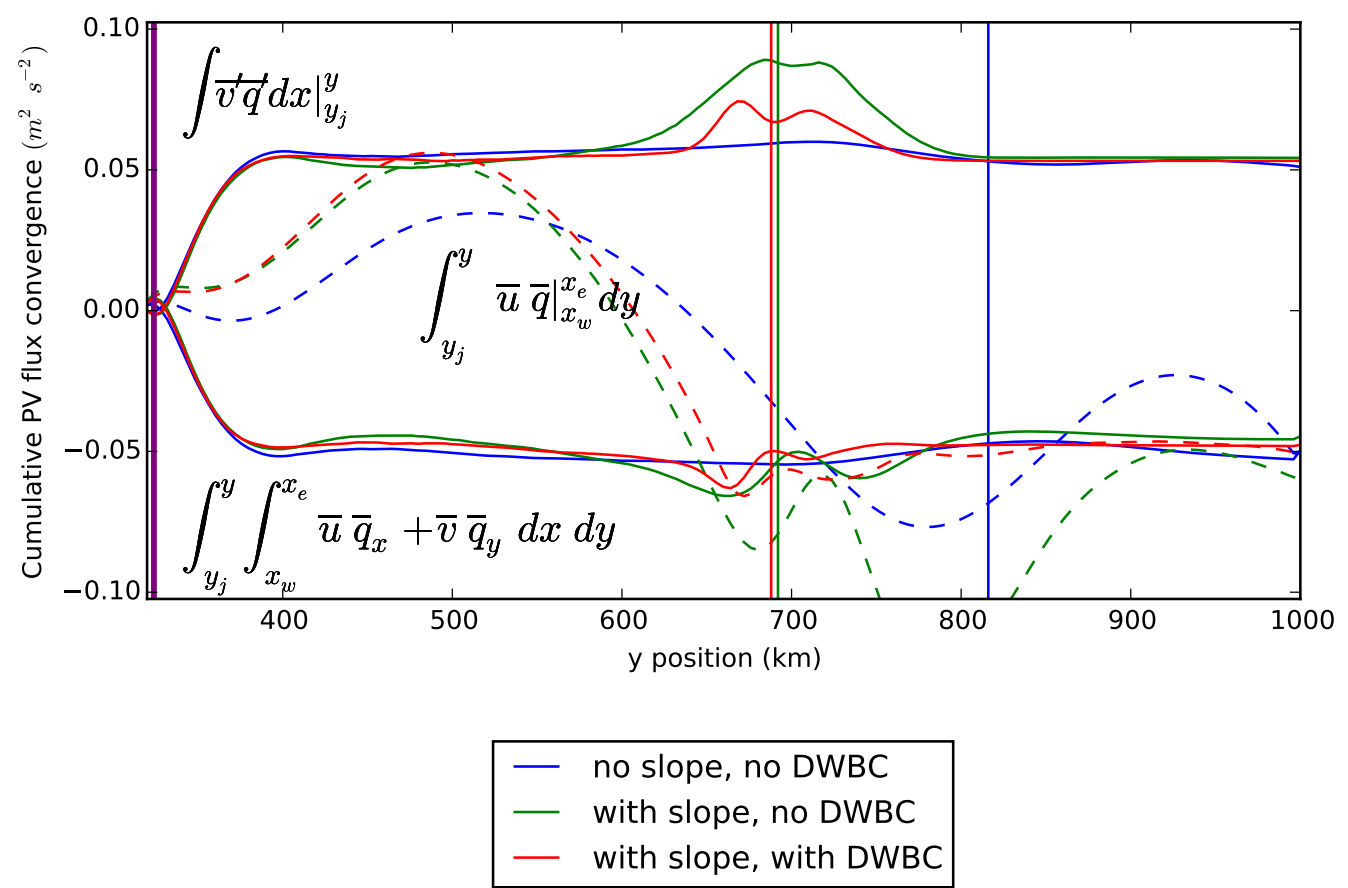

Figure 4-11: Cumulative eddy and mean PV flux convergences in the lower layer (in $m^{2} s^{-2}$ ), integrated northward from the jet axis, $y_{j}$. The eddy PV flux is equivalent to its meridional component, $\left.\int_{x_{w}}^{x_{e}} \overline{v_{2}^{\prime} q_{2}^{\prime}} d x\right|_{y_{j}} ^{y}$. This is balanced by the total mean PV flux convergence: $\int_{y_{j}}^{y} \int_{x_{w}}^{x_{e}} \overline{u_{2}} \cdot \nabla \overline{q_{2}} d x d y$. At the recirculation gyre boundary, depicted for each model configuration by vertical lines, the mean PV flux convergence is dominated by its zonal component, $\left.\int_{y_{j}}^{y} \overline{u_{2}} \overline{q_{2}}\right|_{x_{w}} ^{x_{e}} d y$, which is shown in dashed lines.

down-gradient PV fluxes at the jet center and at the gyre boundary is balanced by the mean PV flux divergence associated with the mean up-gradient PV flux within the NRG region, as illustrated schematically in Figure 4-10.

When a slope is added within the natural extent of the recirculation gyre, this balance is achieved at a more southerly position, where larger eddy PV fluxes down the bathymetric PV gradient support a larger mean PV flux up the large bathymetric PV gradient. This eddy PV flux is an eddy relative vorticity flux, as shown in Figure 4-9, leading to the large negative mean relative vorticity at the base of the slope that closes the recirculation gyre.

When a DWBC is added on the slope, the eddy PV flux down the bathymetric gradient is suppressed. This is likely because the overall PV gradient on the slope is smaller due to the thickness gradient of the DWBC. The extent of the recirculation gyre return flow is also constrained by the presence of the high PV DWBC flow, limiting the potential for up-gradient mean PV flux by the recirculation gyre.

The DWBC flows down the mean PV gradient: it enters the recirculation gyre region at higher PV than it exits with, as it flows from a PV gradient dominated by the bathymetric slope to lower PV as a result of homogenization at recirculation gyre longitudes. This 


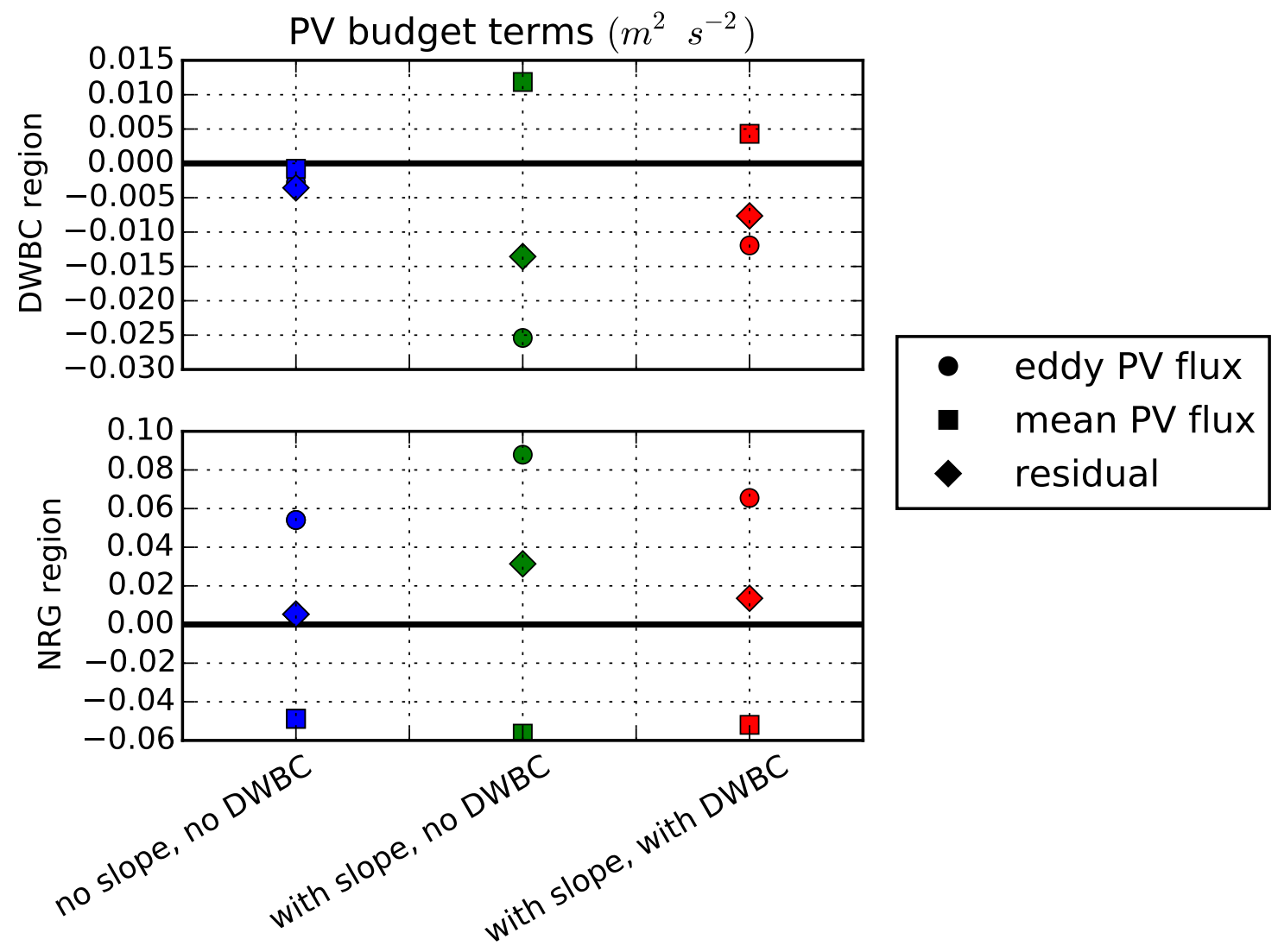

Figure 4-12: PV budget synthesis for model configurations without a slope or DWBC (blue), with a slope, but no DWBC (green), and both a slope and DWBC (red). Budget regions are between $x_{w}$ and $x_{e}$, the length of the recirculation gyre outside of the sponge region. The NRG region extends from the jet axis, $y_{j}$, to the predicted edge of the recirculation gyre, $y_{r}$ and the DWBC region extends from $y_{r}$ to the model's northern boundary. Region boundaries are shown in Figures 4-3 and $4-10$.

completes the full balance that is summarized schematically in Figure 4-10.

In summary, eddy PV fluxes at the DWBC-gyre boundary flux PV down-gradient, removing PV from the DWBC, which flows down the mean PV gradient. The southward eddy PV flux on the slope increases the eddy PV flux convergence in the NRG region, sustaining the mean PV flux divergence associated with the part of the recirculation gyre return flow that crosses onto the high PV slope.

This balance between meridional eddy PV flux convergence and mean PV flux divergence for the recirculation gyre region is shown quantitatively in Figures 4-11 and 4-12. As shown in Figure 4-11, at the gyre boundary the zonal mean PV flux convergence dominates the mean PV flux divergence at the gyre boundary, i.e. $\left.\int_{x_{w}}^{x_{e}} \overline{v_{2}^{\prime} q_{2}^{\prime}} d x\right|_{y_{j}} ^{y_{r}}+\left.\int_{y_{j}}^{y_{r}} \overline{u_{2}} \overline{q_{2}} d y\right|_{x_{w}} ^{x_{e}} \approx 0$. This balance holds particularly well in the case with a DWBC. This balance is more complicated in the case that includes a slope, but no DWBC as some zonal flow develops high on the slope, as can be seen in Figure 4-6. The large mean zonal divergence associated with this 
flow, visible in Figure 4-11, is compensated by mean meridional divergence.

There is a general balance between mean and eddy PV fluxes in the model interior. The magnitude of the mean and eddy terms in both of these regions increase when a slope is added in the lower layer, and decrease when a DWBC is added to the slope, though they are still larger than the case without a slope. However, there is an imbalance between these mean and eddy terms that is largest when the eddy fluxes at the edge of the gyre are largest, as shown in Figure 4-12. This non-negligible residual indicates that steady-state has not yet been reached: integrated $\mathrm{PV}$ is still decreasing in the DWBC region and decreasing in the NRG region.

Unfortunately, we did not include a diagnostic to calculate the rate of change of PV in the model directly, and are not able to infer it from the means and limited snapshots that were computationally feasible to save. To check whether the size of the residual is sensible, we can estimate the mean change in PV that would result from such residuals, $\overline{\Delta q_{2}^{\prime}}$, by

$$
\overline{\Delta q_{2}^{\prime}}=\frac{\text { residual } \times \Delta t}{A} .
$$

Using the orders of magnitude for the case with the largest residual, which is in the balance for the NRG region in the model configuration with a slope in the lower layer, but no DWBC, yields

$$
\overline{\Delta q_{2}^{\prime}}=\frac{0.03 \mathrm{~m}^{2} \mathrm{~s}^{-1} \times 5.6 \text { years }\left(\times 3.15 \times 10^{7} \text { seconds } / \text { year }\right)}{\left(5500 \times 10^{3} \mathrm{~m}\right) \times\left(700 \times 10^{3} \mathrm{~m}\right)}=0.1 \times 10^{-5} \mathrm{~s}^{-1} .
$$

This means that over the model averaging period, the PV has increased by $0.1 \times 10^{-5} s^{-1}$ on average throughout the NRG region. This magnitude is reasonable, given the mean $\mathrm{PV}$ profile shown in Figure 4-6. For reference, the change of PV across the slope is $7 \times$ $10^{-5} s^{-1}$. Notably, as discussed in the previous section, the PV of the homogenized region is not significantly different than the initial PV at the jet axis at the zonal location of jet stabilization. Further estimates of the time rate of change of $q_{2}$, for all model configurations, can be found in the Appendix.

\subsection{Varying model parameters}

As discussed in Waterman and Jayne (2011), increasing the instability of the jet increases the magnitude of its associated eddy PV fluxes and the strength of its recirculation gyres. In Waterman and Jayne (2011), the instability is altered by changing the non-dimensional $\beta$ of the system. Here we change the initial strength of the jet, so that the ratio between the planetary and bathymetric PV gradients remains constant. We vary the inflowing jet strength in the upper layer from 0.36 to $1.44 \mathrm{~m} \mathrm{~s}^{-1}$, which is similar to the range explored in 
the two-layer model in Waterman and Jayne (2011) $\left(0.01\right.$ to $\left.1 \mathrm{~m} \mathrm{~s}^{-1}\right)$. The Waterman and Jayne (2011) two-layer model configurations correspond to non-dimensional beta between 0.02 and 0.6, which overlaps with the Flierl (1987) estimate of the Gulf Stream's nondimensional beta between 0.02 and 0.13 .

The instability of the jet also changes the shape of the recirculation gyres. A more unstable jet creates a recirculation gyre with a larger meridional extent and shorter zonal extent, as illustrated in Figure 4-13a) and b). This is because the more energetic eddies created by a more unstable jet are more effective at homogenizing PV and are able to homogenize to higher PV in the north, faster. Waterman and Jayne (2011) show that the change in zonal extent of the recirculation gyre varies in the same manner as the growth rate of the most unstable mode, consistent with the idea that the zonal length of the recirculation gyre is determined by how quickly eddies grow to an efficient PV fluxing size as they are advected by the jet. As demonstrated in Waterman and Jayne (2011), there is a saturation of this effect as the eddies must also stabilize the system to a greater PV gradient for a more unstable jet.

When a bathymetric slope is added within the natural meridional extent of the recirculation gyre, the meridional extent of the recirculation gyre is limited and the gyre extends in the zonal direction. This effect was negligible in the preceding section, as the slope was placed towards the natural edge of the recirculation gyre, but in the cases displayed in the bottom row of Figure 4-13, the gyre shape and PV budget are changed appreciably.

The basic jet considered in the previous section has a maximum inflowing speed of $1.17 \mathrm{~m} \mathrm{~s}^{-1}$, and a natural recirculation gyre meridional extent of $500 \mathrm{~km}$. When a jet with the same inflowing profile is placed $250 \mathrm{~km}$ from the center of the slope, the recirculation gyre is stretched zonally, and weakened, so that standing meanders in the jet become apparent (see Figure 4-13c). Note that wavy jets tend to have eastwards Stokes's drift within their recirculation gyres, so that particles may not follow the mean recirculation gyre flow in this weakened, wavy state (Charney and Flierl, 1981).

The other case considered here is more unstable, with an inflowing jet speed of $1.3 \mathrm{~m} \mathrm{~s}^{-1}$ and natural recirculation gyre meridional extent of $560 \mathrm{~km}$. The position of jet stabilization moves $540 \mathrm{~km}$ downstream when a slope is added $400 \mathrm{~km}$ from the center of the jet, and the recirculation gyres are stretched zonally by almost $2000 \mathrm{~km}$ (see Figure 4-13d). In this case, the recirculation strength increases.

The zonal stretching of the recirculation gyres caused by the addition of a slope is accompanied by changes in the meridional eddy PV flux at the jet axis. In the cases with an inflowing jet velocity of $1.17 \mathrm{~m} \mathrm{~s}^{-1}$, the cumulative meridional eddy PV flux is suppressed when the jet is placed $250 \mathrm{~km}$ from the center of the slope, compared to the case without a slope, or with a slope $400 \mathrm{~km}$ away, as shown by the grey lines in Figure 4-14. This suppressed eddy PV flux cannot stabilize the jet, and the recirculation gyres do not close 
until the eastern sponge region.

In the more unstable case, with an inflowing jet velocity of $1.3 \mathrm{~m} \mathrm{~s}^{-1}$, the model configuration with a slope in the lower layer does reach the maximum cumulative eddy PV flux of the case without a slope, in fact the maximum is higher in the case with a slope (see black lines in Figure 4-14). In this case, the eddy PV flux at the jet axis does stabilize the jet to its PV gradient and the recirculation gyre is closed within the model interior. However, this maximum is reached farther downstream when the slope is present in the lower layer, leading to the zonal stretching that we observe.

The cumulative meridional eddy PV flux along the jet axis is comprised of three primary stages, as schematized in Figure 4-14. There is an initial gradual slope at the west of the domain, where the eddies are growing to finite amplitude as they are advected by the jet, and then a constant, steeper slope, where the eddies are fluxing PV at a constant rate. Finally, once the jet has been stabilized, the eddies flux PV upgradient at a very low rate, which is evident in the slight decrease in cumulative meridional PV flux after the maximum has been reached.

The discrepancy between model runs with and without a slope is primarily due to a difference in the initial slope of the cumulative meridional eddy PV flux, when the eddies are growing, which indicates that the eddy growth rate is suppressed by the addition of the bathymetric slope. The slope of the cumulative meridional eddy PV flux when the eddies are fluxing PV at an elevated, constant rate are much more similar than during this initial growth period.

Model snapshots of upper layer PV, $q_{1}$, also indicate that stability characteristics of the system are changed by the addition of the slope. As shown in Figure 4-15, when there is a slope in the model lower layer, the meander wavelength and eddy size appear to be smaller than in the comparable case without a slope in the lower layer. We show the upper layer PV field as the eddy characteristics are similar in the upper and lower layer, but the eddies in the vicinity of the jet are more visible in the upper layer PV field because of the PV gradient at the jet axis. 

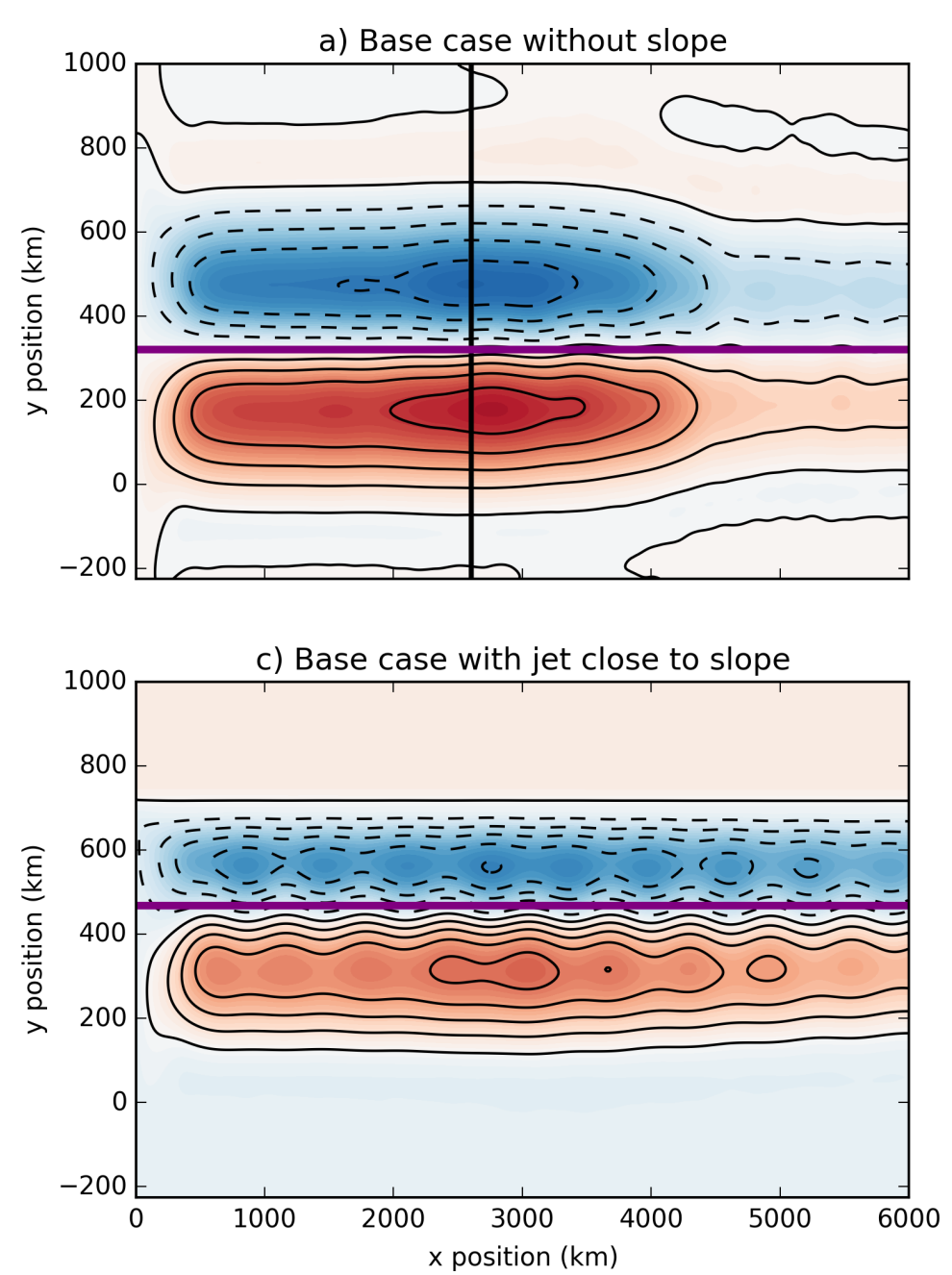

b) More unstable case without slope

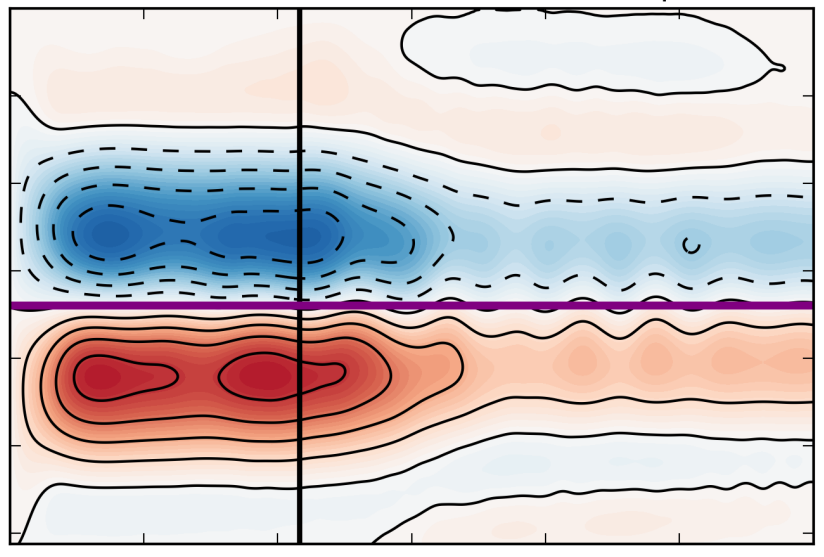

d) More unstable case with slope

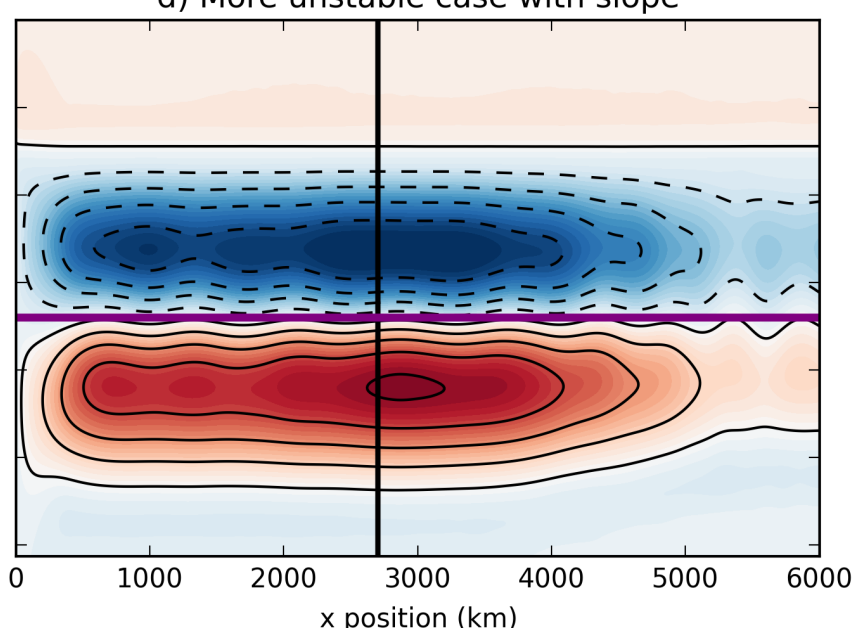

80

60

40

20

$\bigoplus_{0}$

$\mathfrak{3}_{N}$

cos

$-40$

$-60$

$-80$

Figure 4-13: The time mean streamfunction in the lower layer, $\overline{\psi_{2}}$, for model configurations with varying initial jet strength and proximity to bathymetric slope. Top row: no bathymetric slope in the lower layer. Bottom row: center of bathymetric slope is $250 \mathrm{~km}$ away from the jet in c) and $400 \mathrm{~km}$ away from the jet in d). Left column: Initial jet strength is $1.17 \mathrm{~m} \mathrm{~s}^{-1}$ in a) and c). Right column: Initial jet strength is $1.3 \mathrm{~ms}^{-1}$ in b) and d). Vertical black lines depict the zonal position of jet stabilization for each case. Horizontal purple lines indicate the meridional position of the jet axis in each case. 

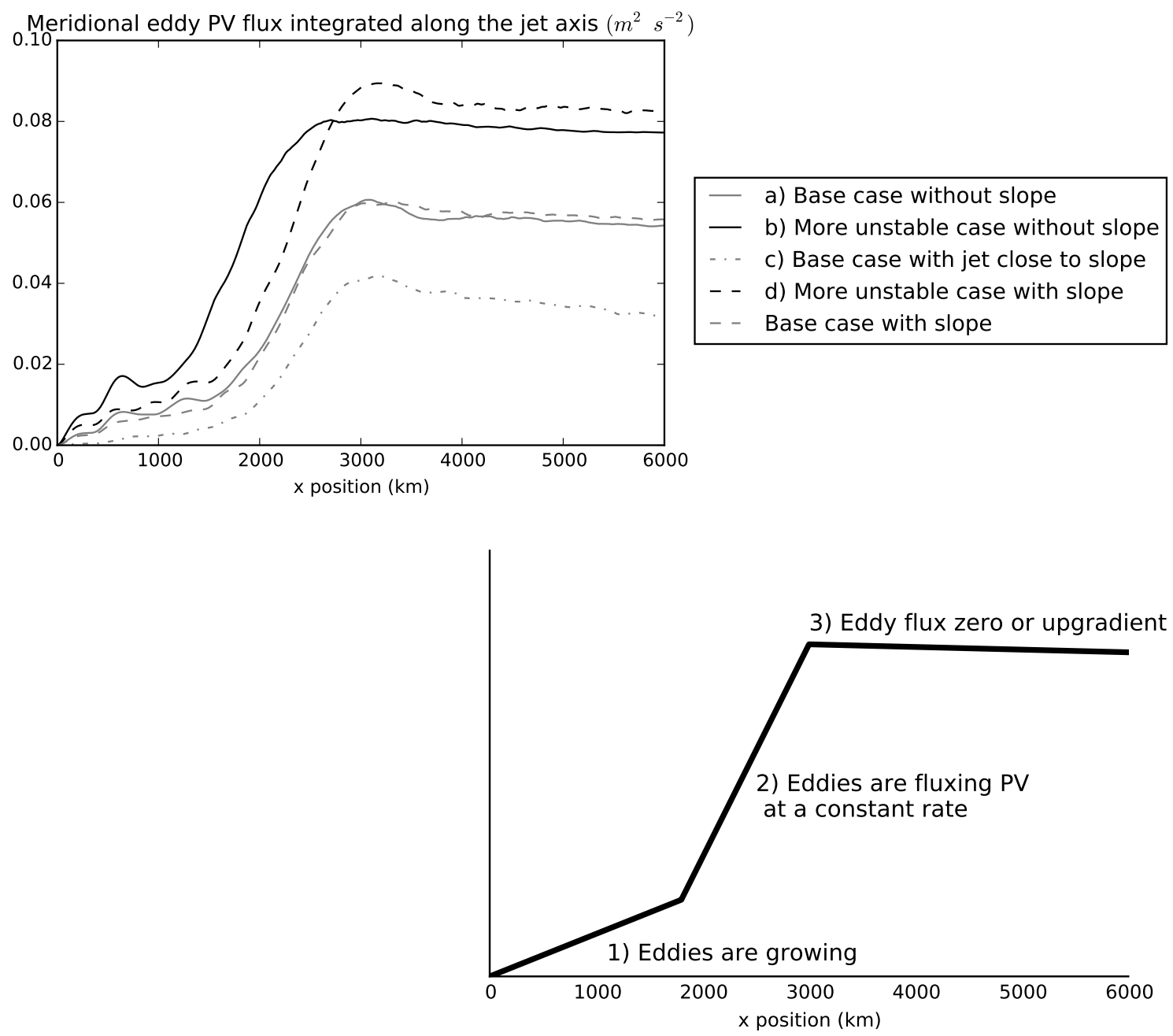

Figure 4-14: Top panel: Lower layer meridional eddy PV fluxes integrated zonally along the jet axis for model configurations shown in Figure 4-13, i.e. $\int_{0}^{x} \overline{v_{2}^{\prime} q_{2}^{\prime}}\left(y_{j}\right) d x$. The jet axis is highlighted by a horizontal purple line in each panel of Figure 4-13. Bottom panel: Schematic showing how the three primary stages of eddy behavior are reflected in the cumulative integral of meridional eddy $\mathrm{PV}$ fluxes along the jet axis.

To investigate this further, we calculated the linear stability characteristics of the model setup. We started from the linearized PV conservation equation

$$
\left(\overline{u_{i}}-c\right) \tilde{q}_{i}^{\prime}+\overline{q_{i y}} \tilde{\psi}_{i}^{\prime}=0
$$

where primes indicate small wave-like perturbations with the form $q_{i}^{\prime}=\tilde{q}_{i}^{\prime}(y) e^{i k(x-c t)}$, and overlines indicate the basic state that we linearize around. We used the definition of $q$ as a function of $\psi$ to form an eigenvalue problem for $\tilde{q}_{i}{ }^{\prime}$, where the eigenvalues correspond to $c$ 's, and the eigenvectors are the corresponding meridional structure of q perturbations, $\tilde{q}_{i}{ }^{\prime}(y)$. We applied this analysis to the jet structures described in this section (Figures 4-13-4-15), 
for the prescribed inflowing jet $u$ and $q$ profiles and for time mean profiles at 1000 and 1600 $\mathrm{km}$ downstream. We calculated the growth rates (i.e. the imaginary component of $c$ ) as a function of zonal wavenumber for the most unstable mode, shown in Figure 4-16, as well as the meridional structure of the perturbations.

We found that the suppressed eddy growth could not be explained using this linear stability analysis. This is primarily because the meridional structure of the most unstable mode is restricted to the meridional width of the jet, which does not intersect with the slope.

In fact, our analysis simply reinforces that the jet is stabilized more slowly when a slope is added to the domain. The inflowing jet profiles have equivalent stability characteristics with or without a slope in the lower layer, as shown in Figure 4-16. The most unstable modes have zonal wavelengths of $30 \mathrm{~km}$ and growth rates of $1 / 2.3$ and $1 / 2.1$ days $^{-1}$ for an inflowing velocity of 1.17 and $1.3 \mathrm{~m} \mathrm{~s}^{-1}$ respectively. The growth rates of the most unstable modes are smaller (and more realistic) for the mean profiles downstream of the jet inflow. The growth rates decrease fastest for the cases without a slope in the lower layer, as the jet is stabilized more quickly. The growth rates also decrease more rapidly for cases with inflowing jet velocity of $1.3 \mathrm{~m} \mathrm{~s}^{-1}$. Interestingly, in the analysis at $1600 \mathrm{~km}$ for cases with a slope in the lower layer, a secondary peak is emerges at smaller scales (higher wavenumber).

The zonal wavelength of the most unstable mode is larger for mean downstream jet profiles at $1600 \mathrm{~km}$. For example, in the case with inflowing velocity of $1.3 \mathrm{~m} \mathrm{~s}^{-1}$ and no slope in the lower layer, the zonal wavelength of the most unstable mode at $1600 \mathrm{~km}$ is 60 $\mathrm{km}$. The meridional structure of the most unstable mode is generally wider at $1600 \mathrm{~km}$ downstream than at $1000 \mathrm{~km}$, but does not intersect with the slope in any of the cases when it is present.

This analysis does not account for what occurs to the instabilities as they grow and become large enough to interact with the slope. This is probably why it does not explain the suppression of eddy growth when a slope is added to the domain. We are also assuming that it is sensible to linearize the jet around its time mean state, and do not take into account zonal changes in jet structure. This analysis indicates that the suppression of eddy growth is likely a non-linear process and investigating this further presents an exciting avenue for future work. 

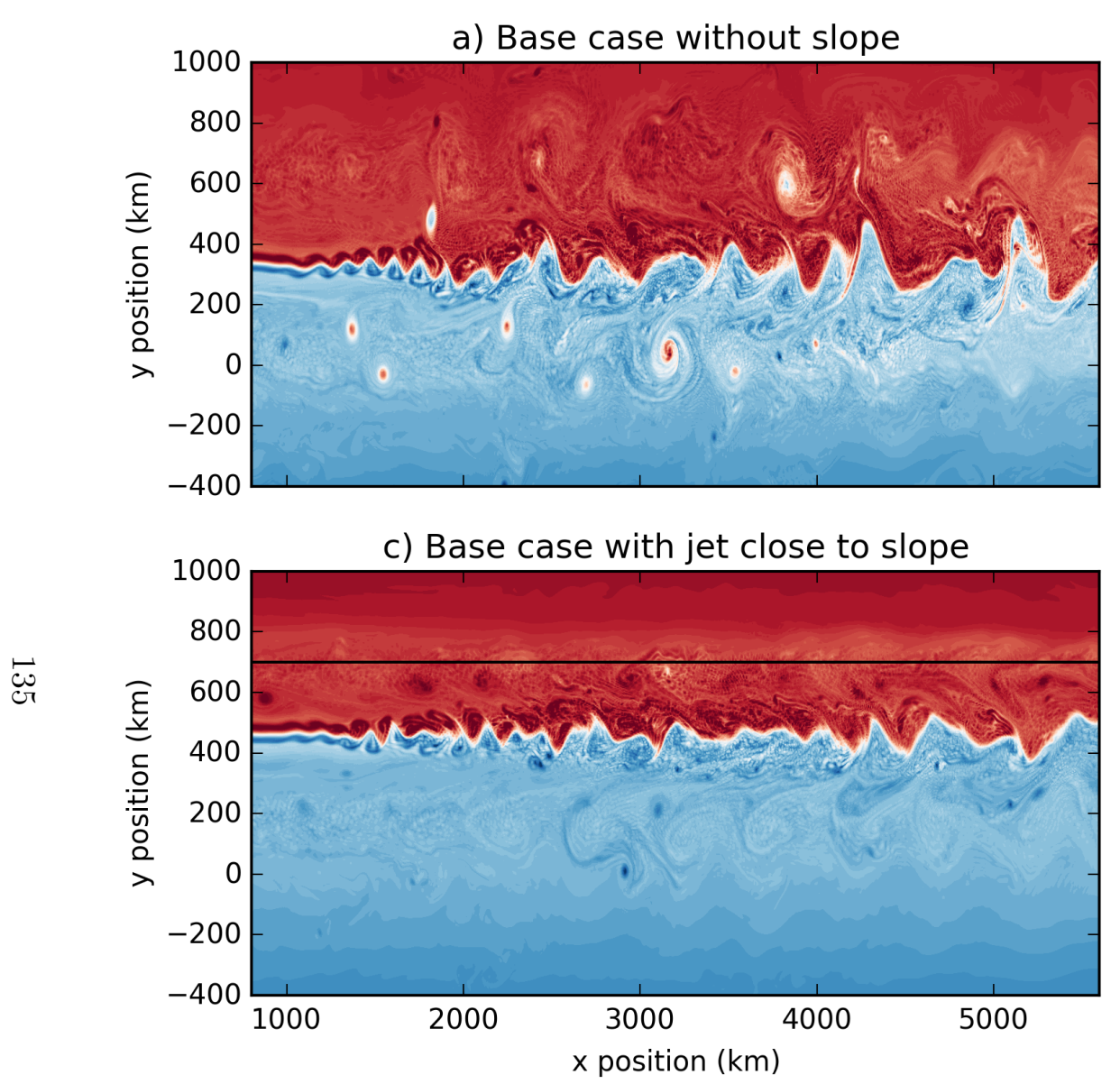

b) More unstable case without slope

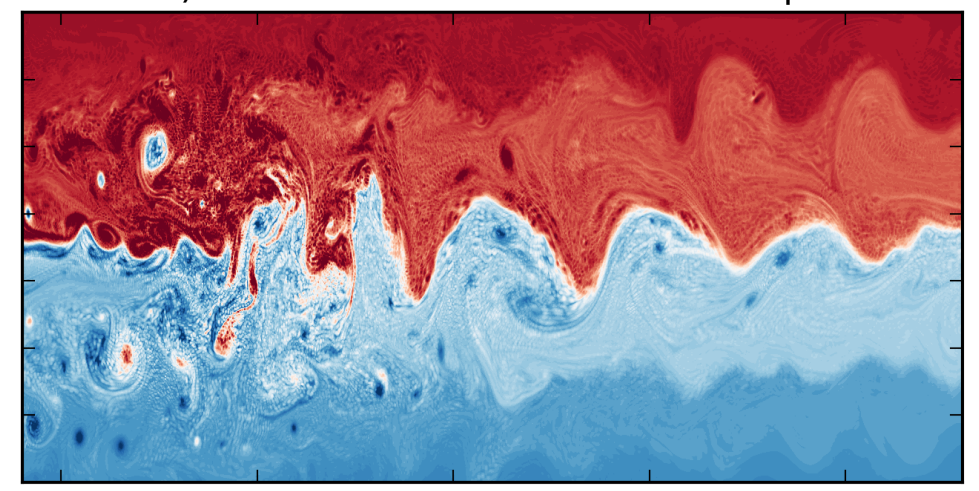

d) More unstable case with slope

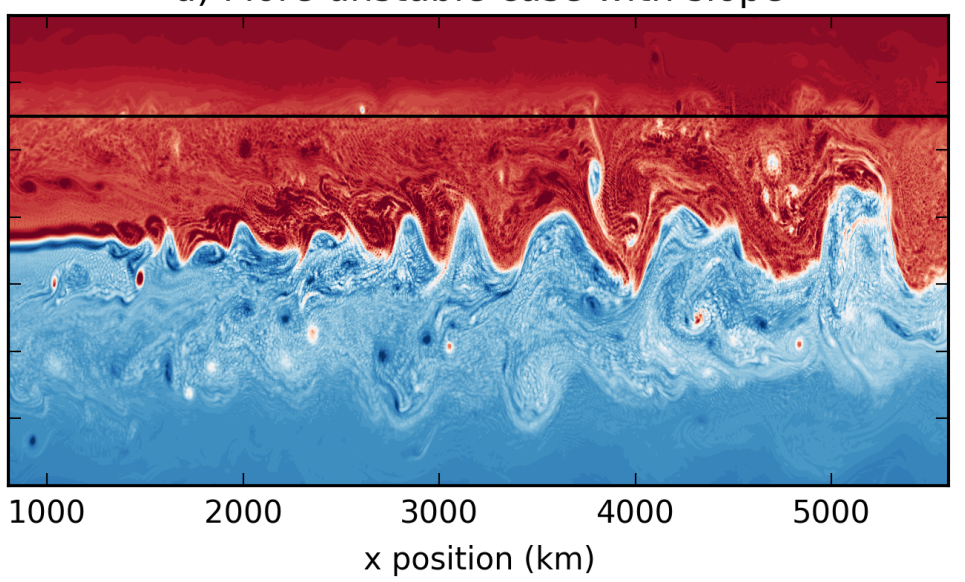

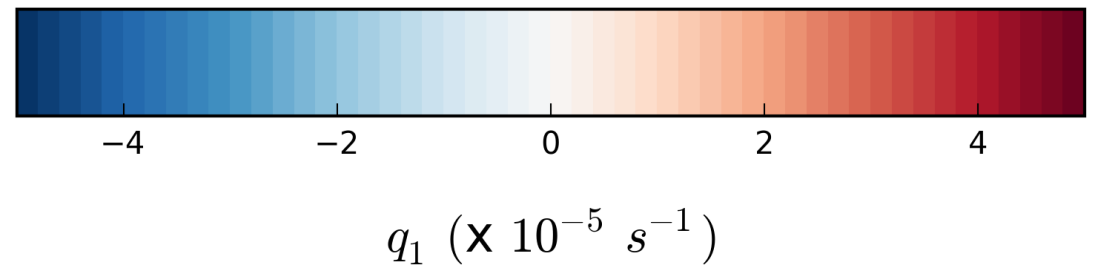

Figure 4-15: Upper layer PV $\left(q_{1}\right)$ snapshots for the model configurations shown in Figures 4-13 and 4-14. Horizontal black lines in c) and d) indicate the meridional position of the center of the slope in the lower layer. 

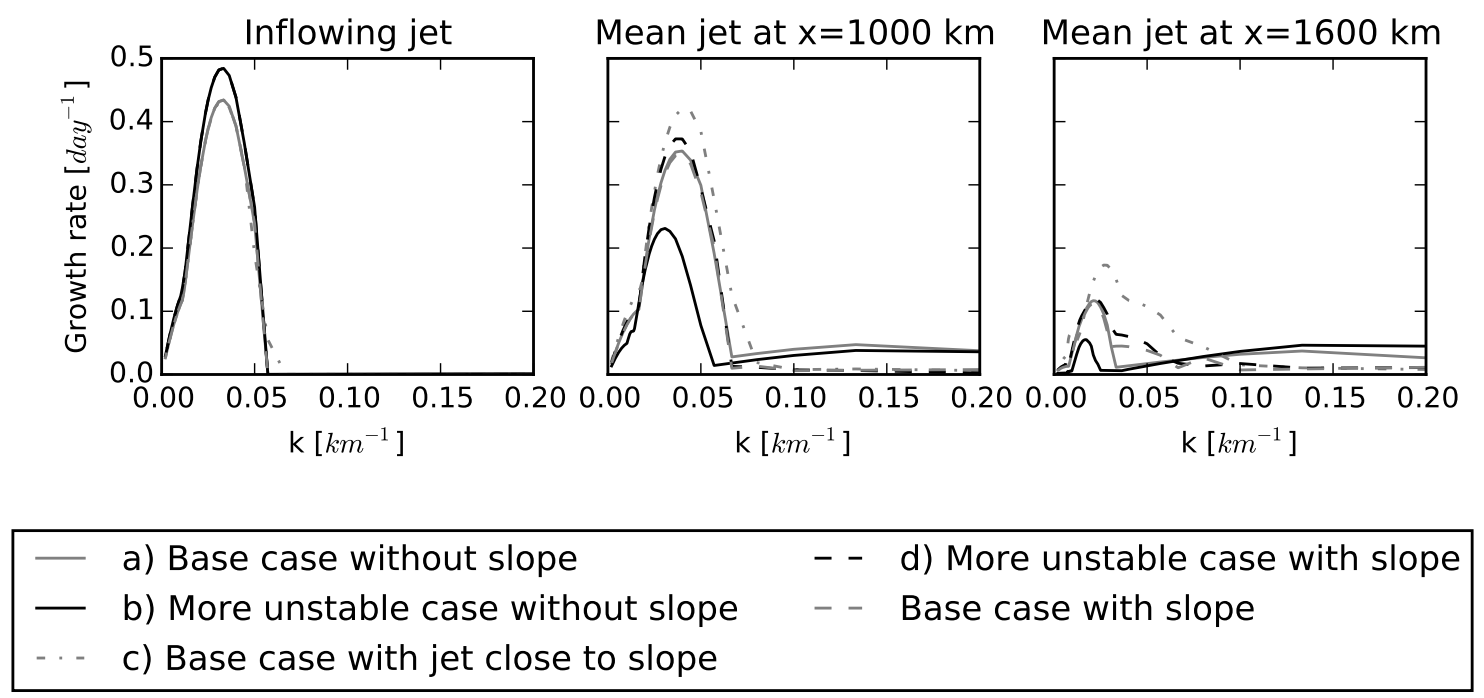

Figure 4-16: Growth rate as a function of zonal wavenumber for the model configurations shown in Figures 4-13 - 4-15 for the inflowing jet profile, and the time mean profiles at 1000 and $1600 \mathrm{~km}$ downstream.

\subsubsection{Varying jet instability}

When the instability of the jet is increased through an increase in the inflowing jet velocity, with the distance between the jet and DWBC held constant, there is a larger thickness gradient in the model lower layer, as shown in Figure 4-17. This results in a larger predicted recirculation gyre extent, since the LHS of Equation 4.3 has a larger initial decrease.

The time-mean profiles displayed in Figure 4-17 reflect that the eddies homogenize farther into the slope for the model configurations with more unstable inflowing jets. The homogenization into the slope is enabled by negative eddy relative vorticity fluxes at the base of the slope which decrease the relative vorticity. As shown in Figure 4-17, the resulting negative relative vorticity at the base of the slope has ever-larger magnitudes for more unstable model configurations. The reduction in relative vorticity by the eddy relative vorticity fluxes extends to the southern edge of the DWBC and can distort the zonal profile of the DWBC.

In Figure 4-17, it appears that the analytic recirculation extent has skill in predicting the maximum in relative vorticity, or the transition between the NRG and DWBC, for model runs in which the slope intersects the natural size of the recirculation gyre. However, as shown in Figure 4-18, the prediction tends to overestimate the position at which the NRG and DWBC velocity profiles merge. The most unstable model configuration presented in Figure 4-18, depicted in the darkest purple, does not follow the same pattern as the less unstable runs as the transition between the NRG and DWBC is less well-defined.

The energetic eddies created by more unstable jets flux more PV down the jet's PV 

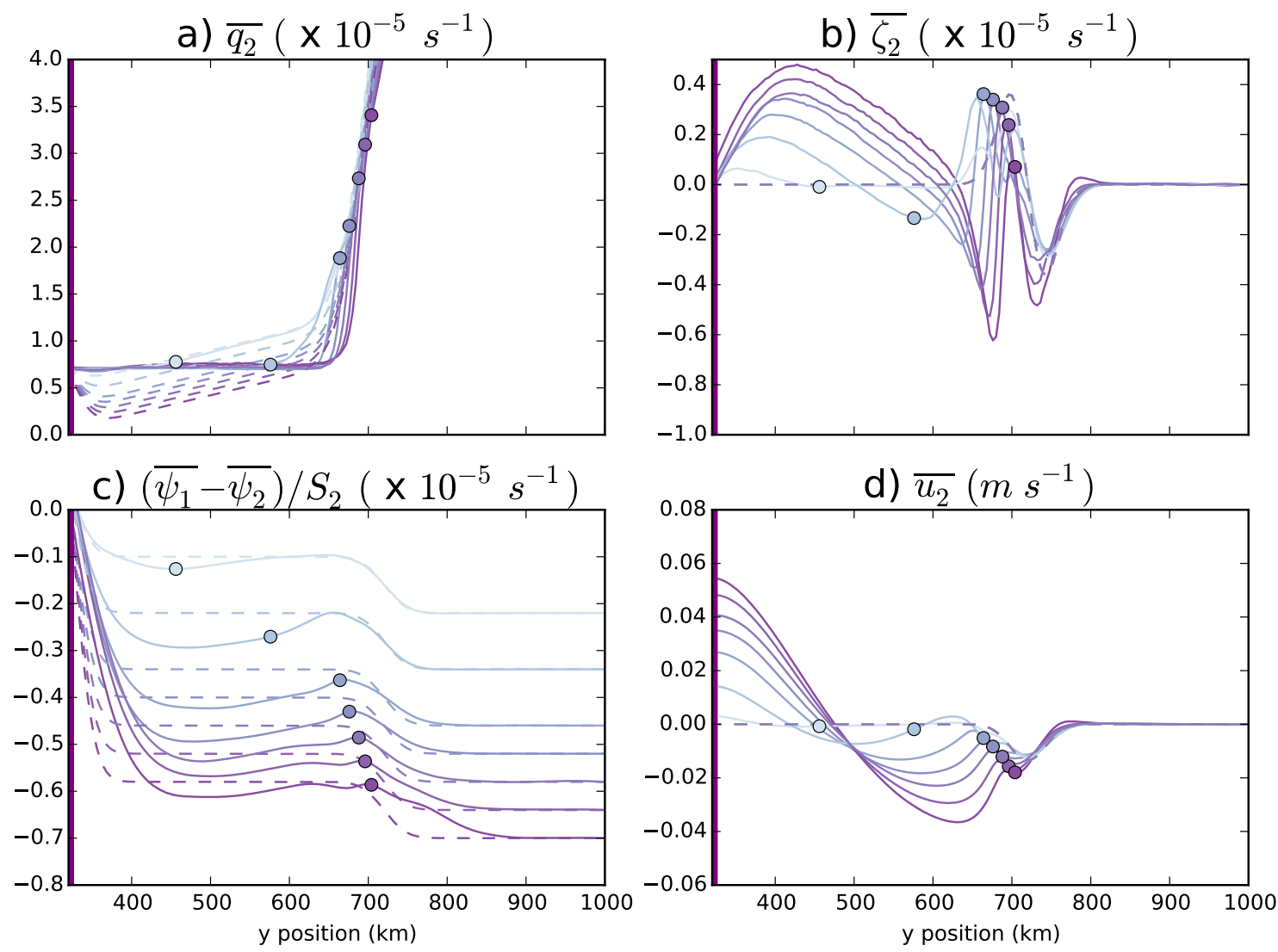

Figure 4-17: As in Figure 4-6, for the model configurations with varying inflowing jet velocities, and hence jet instability. Inflowing jet velocities specified in Figures 4-19 and 4-20 with the same color coding; velocities range from 0.36 to $1.44 \mathrm{~m} \mathrm{~s}^{-1}$ from light to dark. As before, we show properties north of the jet axis, as indicated by the vertical purple line at the left edge of each panel. Solid lines are steady state profiles at the location of jet stabilization, $x_{m}$, and dotted lines indicate initial conditions. Colored points denote the value of each property at the recirculation boundary, $y_{r}$, predicted by the analytic framework presented in Section 4.5.

gradient. By the time they reach the bathymetric slope, they are more energetic than in less unstable cases, causing a larger down-gradient meridional eddy PV flux at the edge of the recirculation gyre. This increased off-slope eddy PV flux is shown quantitatively in the DWBC region PV budget in Figure 4-19. As shown in Figure 4-14 and discussed in the previous section, the cumulative meridional eddy PV flux at the jet remains constant when a slope is added within the natural extent of the recirculation gyre, though the total value is reached farther east when the slope is present. Hence the convergence of eddy PV flux in the NRG region increases with jet instability and is further increased when a slope is added within the natural meridional extent of the recirculation gyre, as denoted by the comparison to the red points in Figure 4-19.

In conjunction with the increase in eddy PV flux in the NRG region, there is an increase in the mean PV flux divergence. This increase occurs as the NRG return flow exits the 


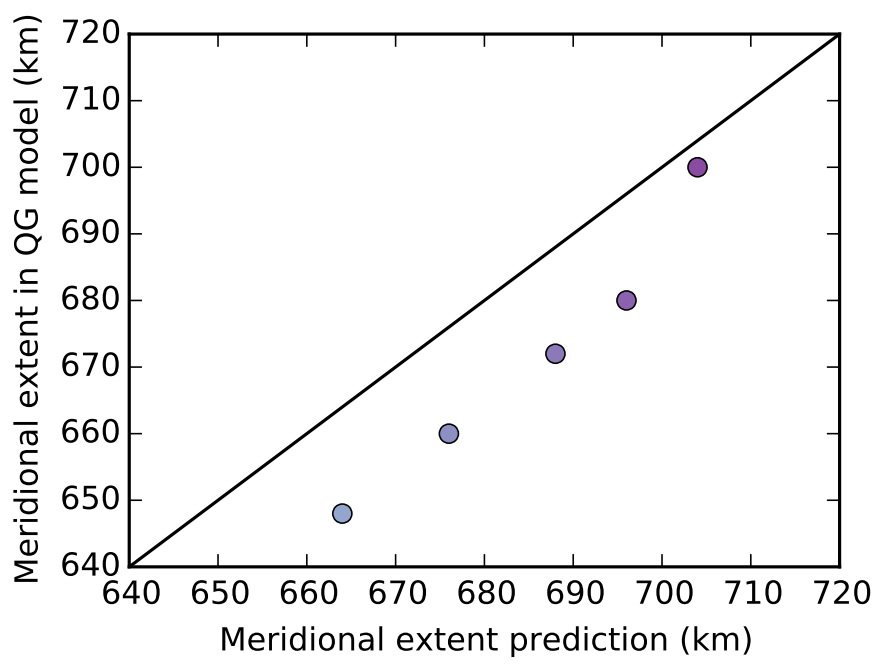

Figure 4-18: Analytic meridional extent prediction against meridional extent in the QG model (both in $\mathrm{km}$ ) for model configurations with varying jet velocities, with a slope and DWBC, in which the recirculation gyre extent intersects with the slope in the lower layer. Colors denote different inflowing jet velocities, as defined in Figure 4-19. Darker colors correspond to more unstable jets. The meridional extent in the QG model is defined as the velocity maximum between the NRG and the DWBC at $x_{m}$. The diagonal black line is the $\mathrm{y}=\mathrm{x}$ line for reference.

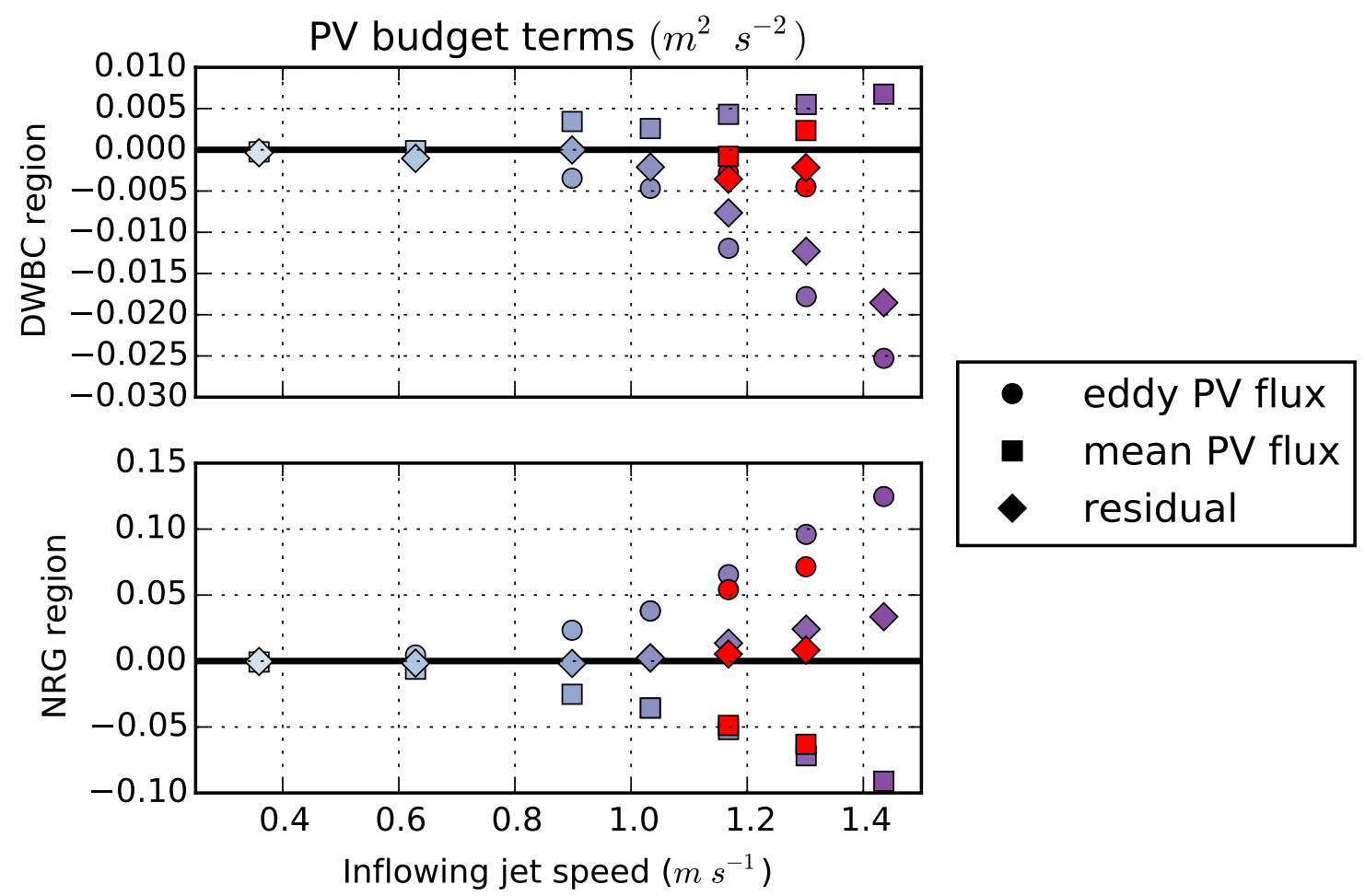

Figure 4-19: PV budget synthesis for model configurations with varying inflowing jet velocities, and hence jet instability. Budget regions are as defined in Figures 4-10 and 4-12. Red symbols denote model configurations without a bathymetric slope or DWBC in the lower layer. 

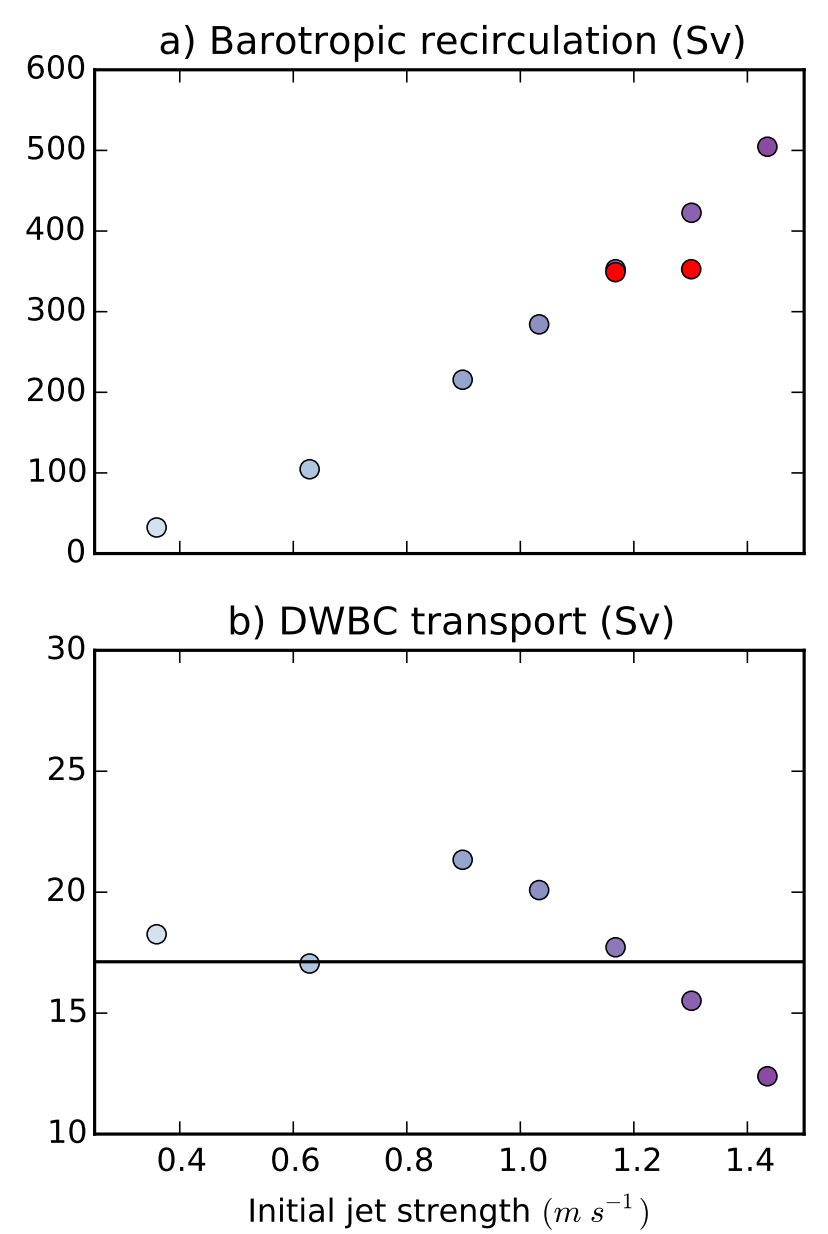

Figure 4-20: Recirculation gyre and DWBC transports for model configurations with varying inflowing jet velocities, and hence jet instability. Red symbols denote model configurations without a bathymetric slope or DWBC in the lower layer. a) Barotropic recirculation strength (sum of upper and lower layer recirculating transport) in Sv. b) DWBC transport in Sv, defined as the zonal transport north of the recirculation gyre extent, $y_{r}$. Horizontal black line indicates the initial DWBC transport.

budget domain at higher speeds with higher PV in more unstable cases. In the DWBC region, the increase in eddy $\mathrm{PV}$ flux divergence is also concurrent with an increase in mean PV flux convergence. However, the changes in mean PV flux are not large enough to balance the increases in eddy PV flux and there is a significant residual, particularly in the most unstable cases. These residuals signal that PV was increasing in the NRG region throughout the averaging period, and decreasing in the DWBC region. The residuals are contextualized further in the final subsection of the Appendix.

The potential consequences of adding a slope in the lower layer are particularly apparent for the case with inflowing jet velocity of $1.3 \mathrm{~m} \mathrm{~s}^{-1}$. When there is no slope in the lower layer, the PV budget does not include significant eddy PV fluxes at the edge of the recirculation gyre, and the residual of the PV budget is much smaller than the mean and eddy PV flux 
terms in the DWBC and NRG regions, as shown in red in Figure 4-19. The addition of the slope causes a large increase in the eddy PV flux at the base of the slope and the recirculation strength is increased by the presence of the slope (see Figure 4-20a), as the eddy PV fluxes make a significant contribution to the eddy PV flux convergence forcing of the gyre. This is in contrast with the base case described in previous sections, with an inflowing velocity of $1.17 \mathrm{~m} \mathrm{~s}^{-1}$, for which there is a smaller change in eddy PV flux and residual in the PV budget for the NRG region, and the strength of the recirculation is not significantly altered.

The eddy PV flux at the base of the slope has consequences for the DWBC. Larger eddy PV fluxes mean more stirring of DWBC origin water with the water south of the slope, and as the recirculation gyre homogenizes further into the slope the zonal extent of the identifiable DWBC decreases, as shown in Figure 4-17. If we define DWBC transport as the zonal transport north of the boundary with the NRG, the magnitude of the DWBC transport decreases as the instability of the gyre increases, as shown in Figure 4-20b. So, with increasing jet instability, the eddy PV fluxes at the interface between the NRG and the DWBC increase, and the DWBC transport is reduced.

\subsubsection{Varying the distance between the jet and the slope}

In this section, we describe the effects of bringing the jet closer to the bathymetric slope for a jet with the base case inflowing velocity of $1.17 \mathrm{~m} \mathrm{~s}^{-1}$. As discussed previously, and shown in Figure 4-14, if the jet is brought sufficiently close to the slope, the cumulative meridional eddy PV flux at the jet axis is suppressed and the recirculation gyre is stretched so far zonally that it is closed in the eastern sponge layer. The model configurations explored here are in this parameter regime. This parameter regime is potentially relevant to the observed NRG as the bathymetric slope at the Tail of the Grand Banks may play a similar role as the eastern sponge by providing closed PV contours to close the recirculation gyre.

The suppression of the jet's cumulative eddy PV fluxes by proximity to the slope renders the analytic framework for predicting the meridional extent of the recirculation gyre inapplicable. Hence, for this analysis, we define the boundary between the NRG and the DWBC as the velocity maximum between the NRG and the DWBC at $x_{m}$. As shown in Figure 4-21, when the jet is close to the slope, the eddies do homogenize farther into the slope. As before, this is facilitated by negative relative vorticity at the base of the slope. The magnitude of the relative vorticity minimum at the base of the slope increases as the currents are brought closer together. However, the change in meridional extent is small in comparison to the changes in meridional distance between the currents because the bathymetric slope's PV gradient is much larger than the PV gradient at the jet axis, and because eddy PV fluxes are suppressed by the proximity of the jet to the slope.

Both eddy PV fluxes at the jet axis and at the base of the bathymetric slope are suppressed when the jet is brought closer to the slope, as shown in Figure 4-22. As a consequence 

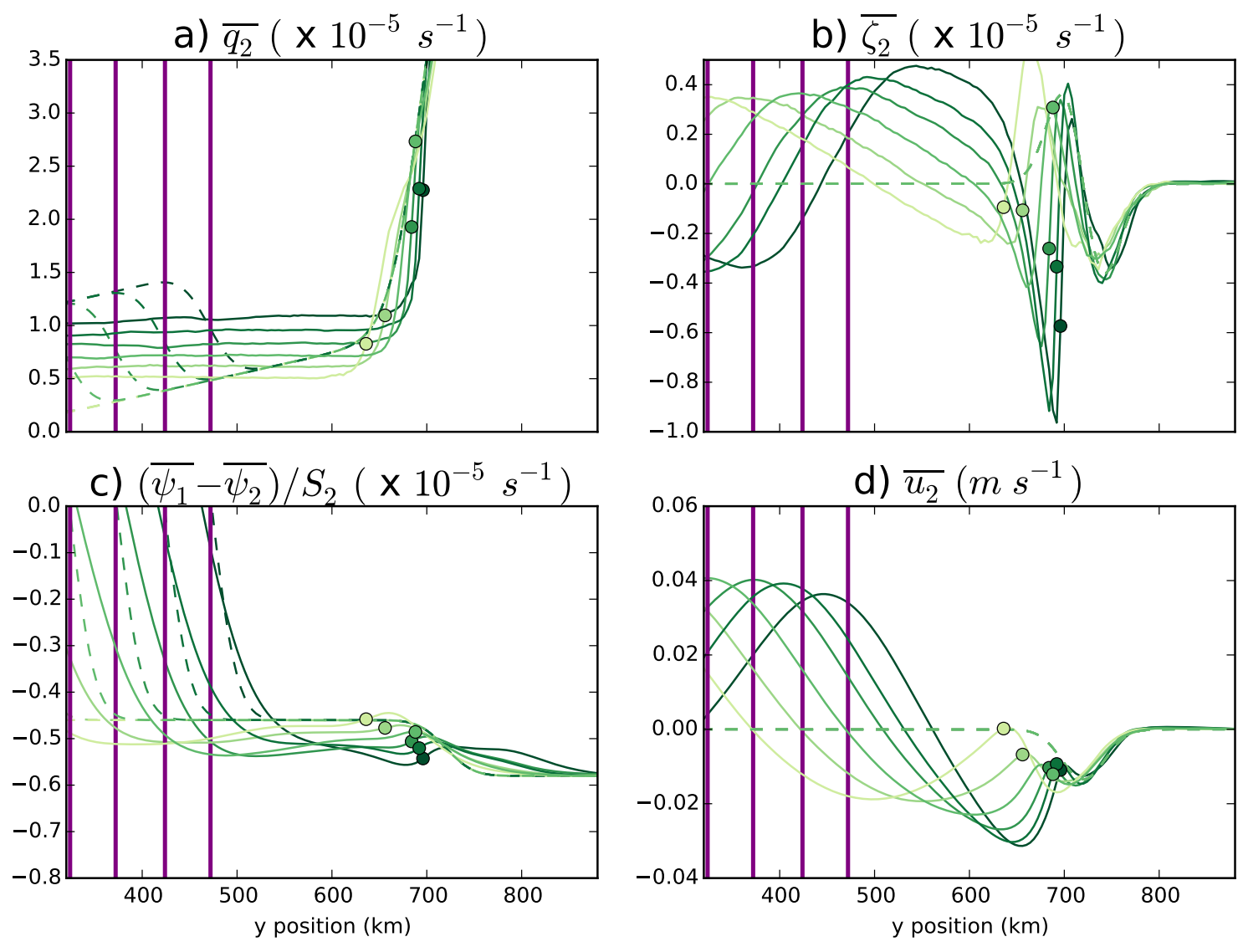

Figure 4-21: As in Figure 4-6, for model configurations with varying distances between the unstable jet and DWBC. Colors correspond to distances specified in Figures 4-22 and 4-23, ranging from 250 $\mathrm{km}$ to $450 \mathrm{~km}$ from dark to light. As before, solid lines are steady state profiles at the location of jet stabilization, $x_{m}$, and dotted lines indicate initial conditions. Colored points denote the value of each property at the recirculation boundary, $y_{r}$, which is defined here as the velocity maximum between the NRG and the DWBC at $x_{m}$. Vertical purple lines denote the meridional positions of the jet axis for each model run.

of decreased eddy PV flux convergence in the NRG region, the barotropic recirculation strength decreases with proximity to the jet, as shown in Figure 4-23a. Note that the differences in recirculation strength reported here are on the order of $50 \mathrm{~Sv}$, whereas the changes associated with changing the jet inflowing velocity are on the order of $500 \mathrm{~Sv}$. This decrease in recirculation strength is also reflected in the decrease of mean PV flux in the NRG region as the currents are brought closer together.

As in the previous sections, there is a non-negligible residual which increases with the magnitude of eddy PV fluxes. This residual is in the same sense as previously reported: the $\mathrm{PV}$ in the NRG is increasing and the PV in the DWBC region is decreasing.

The DWBC transport decreases with proximity to the jet, since we define the transport as all zonal transport north of the NRG-DWBC boundary. However, proximity between the jet and the DWBC can create a barrier to stirring between the NRG and DWBC, as there 


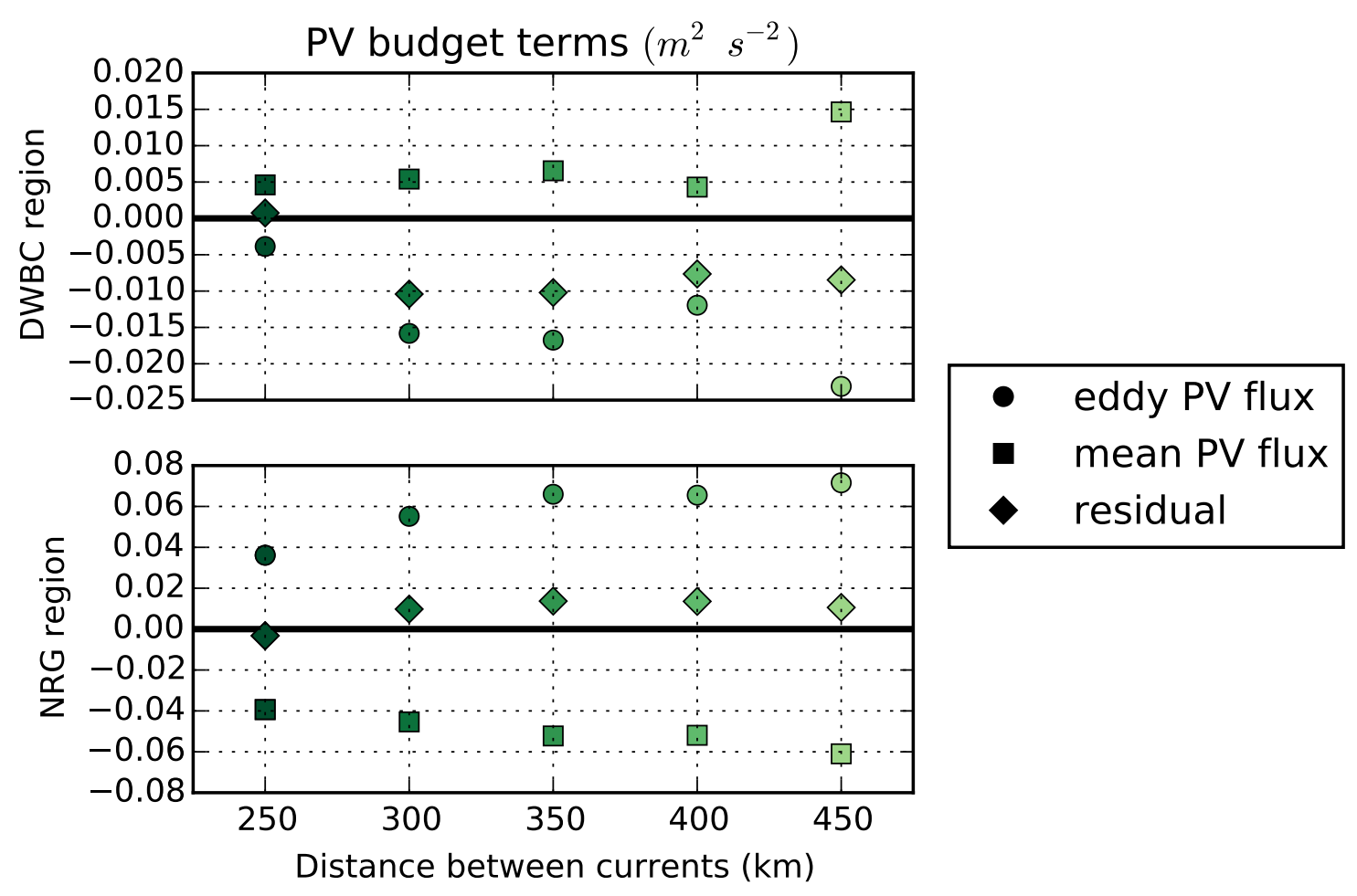

Figure 4-22: PV budget synthesis for model configurations with different distances between the unstable jet and DWBC. Budget regions are as defined in Figures 4-10 and 4-12.

is a steep PV gradient between them with suppressed eddy PV flux across it.

\subsection{Discussion}

We use an idealized two-layer quasi-geostrophic model to investigate the adjustment of a recirculation gyre to a bathymetric slope at depth. The recirculation gyre is constrained meridionally by a bathymetric slope when the slope is closer to the jet axis than the natural width of the recirculation gyre. We are able to predict the meridional extent of the gyre using the assumption that the meridional integral of PV is conserved, as in Jayne et al. (1996), and find that the recirculation gyre can homogenize PV into the slope.

The PV budget of the recirculation gyre is a balance between an eddy PV flux convergence and a mean PV flux divergence. When a slope is added in the lower layer, eddy PV fluxes at the base of the slope increase the eddy PV flux convergence in the recirculation gyre region, and the mean PV flux divergence also increases. However, in the model runs presented here, and particularly the higher energy ones, there is a non-negligible residual of the same sign as the eddy PV flux, indicating that steady state has not been reached.

The eddy PV fluxes at the base of the slope impact the DWBC which flows westward on the bathymetric slope by stirring its water properties with those of the NRG. This effect 

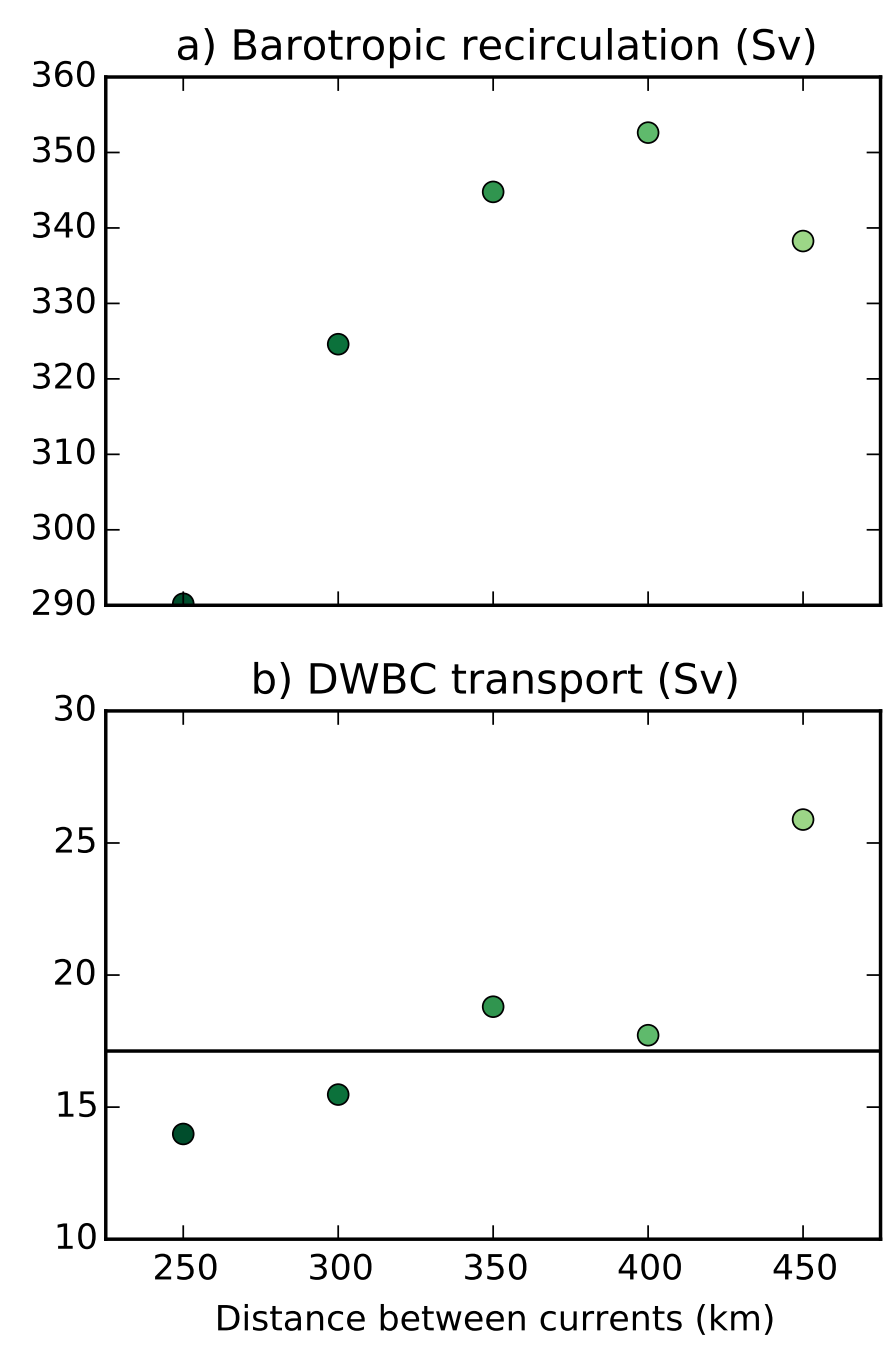

Figure 4-23: Recirculation gyre and DWBC transports for model configurations with different distances between the unstable jet and DWBC. Property definitions are as in Figure 4-20, and horizontal black line in $\mathbf{b}$ ) indicates the initial DWBC transport.

becomes more pronounced when the instability of the jet is increased. Additionally, the boundary between the NRG and DWBC is further north when the jet is more unstable, reducing the DWBC transport.

Our analysis focuses on the parameter space in which the size of NRG is limited by seafloor bathymetry, as indicated by previous studies (Hogg, 1992; Lozier, 1997; Zhang and Vallis, 2007). The co-existence of the NRG and DWBC at depth was observed by Hogg (1983) and Lagrangian studies suggest a mass flux between them (Bower et al., 2009; Gary et al., 2011). Further, Andres et al. (2015) showed that deep cyclones can stir tracer between the DWBC and the interior at Line W, just south of the NRG.

However, eddy PV fluxes have not been reported in observations or models at the edge of the NRG. Xu et al. (2015) found off-slope eddy tracer fluxes in a high-resolution model 
at the Flemish Cap, just north of the Tail of the Grand Banks. This indicates that off-slope eddy fluxes could be significant more generally where there are large eddy fields, and our model framework could be used to interpret the dynamics of this interaction further. Bower and Hogg (1992) and Bower and Hogg (2000) caution that model-data comparisons of eddy fluxes are particularly challenging as eddy fluxes are both difficult to measure and sensitive to model particularities.

It is unlikely that our model recreates the same broad swath of DWBC water travelling downstream observed in Gary et al. (2011), because of the sense of the circulation of the NRG. Once DWBC water is stirred into the interior, it circulates with the recirculation gyre. Though the properties are spread throughout the gyre, the mean velocity is in the direction of the Gulf Stream in the interior. This is similar to the effect in the simple model presented in Hogg et al. (1986) to explain the tongue of DWBC water in the interior due to stirring with the NRG.

For several model configurations, we find a change in DWBC transport as it reaches the longitude of the NRG. This suggests a rearrangement of mass that may also occur in reality as the DWBC rounds the Tail of the Grand Banks and reaches the NRG. Lagrangian studies implicate the Tail of the Grand Banks as a location at which there is heightened stirring between the DWBC and the interior (Bower et al., 2009; Gary et al., 2011), and it is receiving growing attention as a climatically significant transition point (Buckley and Marshall, 2015).

In many of our model configurations, there is no zero crossing of the zonal velocity between the DWBC and NRG. This potential merging of the DWBC and NRG has significant consequences for the interpretation of moored boundary arrays that monitor the DWBC, where the DWBC is hard to define because of its proximity to the Gulf Stream and NRG (e.g. Line W, Toole et al., 2011). At Line W, DWBC transport is reported as the maximal equatorward transport integrated off-shore from the inshore-most mooring at the $2200 \mathrm{~m}$ isobath. In 2008, the moored array was extended to the $4500 \mathrm{~m}$ isobath and the inclusion of this mooring in the analysis leads to an increase in DWBC transport over the calculation using the initial 5 moorings (John Toole, personal communication). This suggests that the DWBC and NRG transports may be indistinguishable at depth.

The reported consequences for the DWBC are generally dependent on the position, width and strength of the DWBC. For example, a DWBC that is positioned farther up-slope than in the configurations presented here may not merge with the NRG. Because we find that it responds passively to the recirculation gyre eddies, we hypothesize that regardless of its position on the slope, the DWBC will be exposed to eddy PV fluxes, which will cause stirring with nearby water masses. We have not explicitly explored the sensitivity to this variable due to the inherent complexity in creating controlled experiments when varying position of the DWBC on the slope, which affects its transport. 
Our model also has implications for the dynamics of the NRG. We found that proximity to the bathymetric slope can suppress eddy PV fluxes at the jet axis and stretch the recirculation gyre zonally. For a given inflowing jet velocity, the cumulative eddy PV flux will eventually reach the same value, as the eddy PV fluxes must stabilize the jet to the same PV gradient. However, if the recirculation gyre is stretched zonally sufficiently, it is closed in the eastern sponge region and there is less cumulative eddy PV flux in the model interior. This balance may be relevant to the real world NRG as the Tail of the Grand Banks could be acting to close mean PV contours, similar to the mechanism suggested by Zhang and Vallis (2007). In this parameter space, the DWBC is less affected by the jet when it is brought closer to the slope, as the eddy PV fluxes are suppressed and there is a larger PV gradient between them.

We also found a model case in which the off-slope eddy PV fluxes are large enough to cause an increase in recirculation strength, however there is no evidence that this is occurring in nature.

Our idealized quasi-geostrophic model framework allows us to isolate the dynamics of the system. However, this simplicity also limits the scope of our results. For example, small irregularities in bathymetry, submesoscales and bottom boundary layer dynamics may play an important role in reality and are not resolved in our model. The QG approximation is also limiting for this application because it does not allow vanishing layer thicknesses or very steep topography. This limits the model DWBC to one layer, which is analogous to the deep overflow water component of the DWBC.

Observations and past modeling work indicate that a two layer representation of the DWBC is preferable because of the differences in the water mass components of the DWBC (Pickart and Smethie, 1993; Spall, 1996a). In particular, the present model lacks a layer analogous to the intermediate Labrador Sea water, which would likely complicate the dynamics of the system because of its low PV signature. In Spall (1996a), the intermediate Labrador Sea water layer sheltered the deep layer from layer thickness changes when the DWBC flows under the Gulf Stream. In our model configuration, the interaction between the DWBC and the NRG would likely be more significant in such an intermediate layer, where the eddy energies are larger.

Our focus was on a field of eddies forced by an unstable jet, but the effect of each individual eddy on cross-slope exchange was not explored. Recent work by Cherian (2016) studied the effect of individual eddies on cross-shelf exchange, using the beta-effect to propel eddies into a slope. He emphasizes the differences in behavior for anticyclonic and cyclonic eddies and finds a cross-slope stirring mechanism that results in subsurface exchange. Our vertical resolution is limiting in that we cannot reproduce this mechanism and though we have not analyzed this carefully, we have not found differences in behavior between anticyclonic and cyclonic eddies. Our focus was on deep exchange affecting the DWBC forced by 
an unstable jet and his on cross-shelf exchange by individual eddies, so that there remain many interesting questions at the interface of these two studies.

In this work, we explored the dynamics of interaction between the Northern Recirculation Gyre of the Gulf Stream and the Deep Western Boundary Current. We found plausible mechanisms that constrain the extent of the NRG as well as eddy PV fluxes that cause the DWBC to stir with the NRG. These mechanisms may shape the meridional overturning circulation in the western North Atlantic, with potential feedbacks on the climate system, however their relevance in nature remains to be quantified. 


\subsection{Appendix}

\subsubsection{Numerical method}

As described in Waterman and Jayne (2011), the model solves the barotropic and baroclinic equations, which are equations for $\left(q_{1} / S_{1}+q_{2} / S_{2}\right)$ and $\left(q_{1}-q_{2}\right)$ respectively. Details on the numerical method can be found in Waterman and Jayne (2011) and Jayne and Hogg (1999). The time stepping is done using a third-order Adams-Bashforth scheme (Durran, 1991), integration in time and space is done using an Arakawa A-grid and advection terms are calculated using the Arakawa (1966) vorticity-conserving scheme. To solve for the streamfunction at each time step, the relative vorticity is inverted using the generalized Buneman algorithm (Adams et al., 1988). Eddy flux terms are accumulated while the model is running, so that there are contributions from each time step.

The sponge layers are as in Waterman and Jayne (2011), 100 grid points wide in the west, and 200 in the south and east of the domain. In this model configuration, there is no sponge layer in the north of the domain, mimicking the continental boundary. In each sponge layer, the linear friction coefficient, $R$, is ramped up linearly from the background value to its maximum at the domain boundary. The non-dimensional value of the friction coefficient in the sponge layers is shown in Figure 4-24.

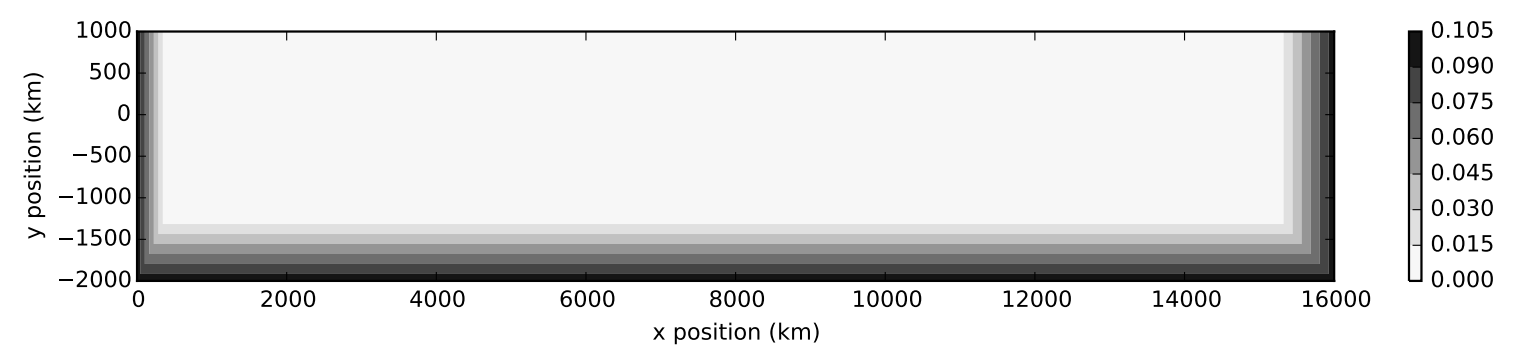

Figure 4-24: Nondimensional value of sponge region linear friction coefficient, $R_{\text {sponge }}$, in the model domain. These sponge regions are present in both model layers. Southern and western sponge region are each $800 \mathrm{~km}$ wide. The western sponge region is $400 \mathrm{~km}$ wide.

\subsubsection{Differences with Waterman and Jayne (2011) model setup}

There are several notable differences between our model setup and that used in Waterman and Jayne (2011) beyond those intrinsic to our scientific questions, the addition of a slope and DWBC in the model lower layer.

In our model framework, we have decreased the time step from $0.04 \mathrm{ndu}$ (non-dimensional units) to $0.025 \mathrm{ndu}$. We have added viscosity, as described in Section 4.3, to suppress gridscale noise and smooth time-averaged fields and fluxes. We also added dependence of the linear friction on gradients in the linear friction coefficient, $R$, i.e. we changed the term in 
equation 4.4 from $R \nabla^{2} \psi$ to $\nabla \cdot(R \nabla \psi)$.

The geometry of the model domain is also distinct. Our domain is longer in the zonal direction: $400 \mathrm{ndu}$ instead of $150 \mathrm{ndu}$. This is because the addition of the slope in the lower layer can stretch the recirculation gyres in the zonal direction, and we wanted to focus on cases where the recirculation gyres are closed within the model interior. The meridional length of our domain is shorter than in Waterman and Jayne (2011) (75 ndu instead of $150 \mathrm{ndu}$ ), since the slope restricts the meridional extent of the gyre. This shortening in the meridional direction is an attempt to save computational time and initially showed little qualitative change in behavior. However the final subsection in this appendix draws attention to likely contamination from the southern sponge because the meridional extent of the model is too small.

\subsubsection{Sensitivities to fixed model parameters}

Sensitivity of standing meanders in the model to various sponge parameters was examined in an effort to suppress them further. We found no significant dependence of the size of standing meanders on the presence of a sponge in the north of the model domain, a doubling of the western sponge layer strength, or the addition of the spatial dependence on the linear friction coefficient to the linear friction term. However, this testing was not exhaustive, and remains to be investigated further.

We found that the model behavior is sensitive to the value of the constant viscosity parameter, $A$, which determines the size of the diffusion intended to suppress grid-scale noise. Significantly, an increase in $A$ decreases the relative vorticity of the DWBC, which is not realistic. Large viscosity also suppressed homogenization into the slope, as sharp gradients in relative vorticity were smoothed out by the viscosity. We ran the model in the base case configuration with non-dimensional values of $A=3 \times 10^{-4}, 1 \times 10^{-4}, 3 \times 10^{-5}$ and $1 \times 10^{-5}$, which correspond to dimensional values of $13,4.3,1.3$ and $0.4 \mathrm{~m}^{2} \mathrm{~s}^{-1}$, respectively. Our chosen value of $A=1.3 \mathrm{~m}^{2} \mathrm{~s}^{-1}$ does not smooth out the DWBC but does suppress grid-scale noise.

We also found that the shape of the recirculation gyres is sensitive to the value of this parameter, with higher viscosity lengthening the recirculation gyres in the zonal direction, as shown in Figure 4-25. This is likely because the viscosity smooths the relative vorticity of the jet, decreasing the instability of the jet as well as the eddy fluxes. This change is analogous to the sensitivity of gyre shape to jet instability discussed in Section 4.7. Note that these averages are over the final 5 years of an 8 year model run, and the model is not in steady state, as discussed in the main text. 

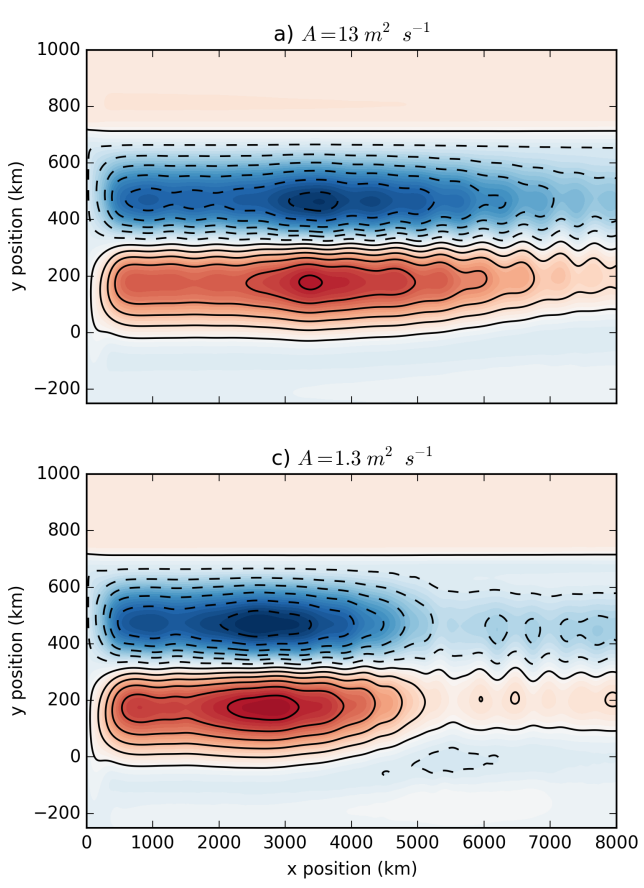

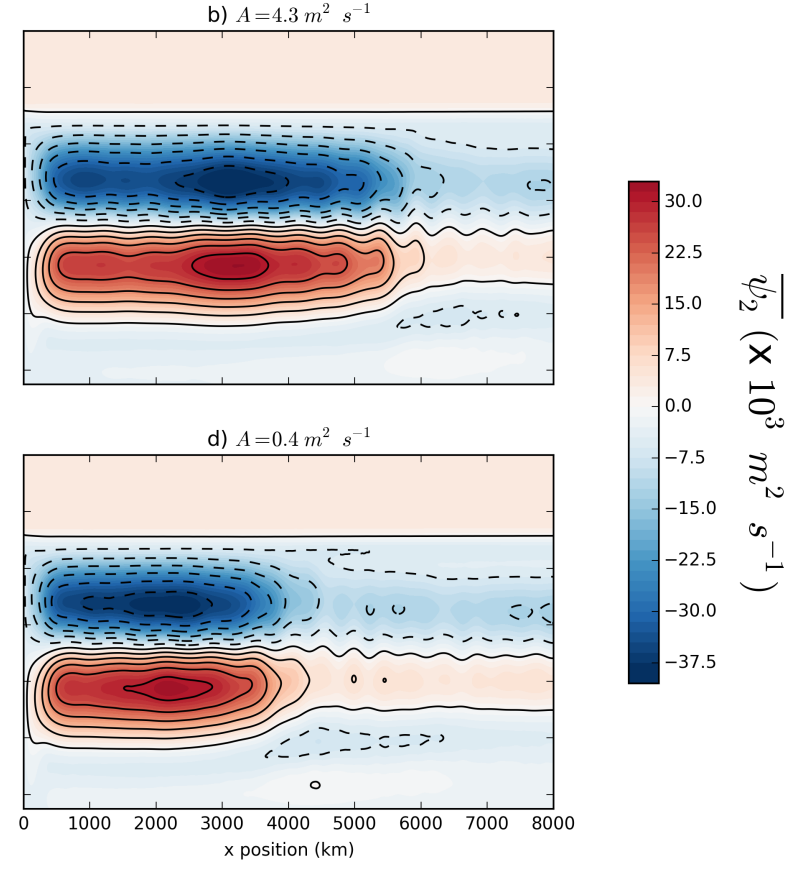

Figure 4-25: Mean lower layer streamfunction, $\overline{\psi_{2}}$, for different values of the viscosity coefficient, $A$. There is also a standard slope and DWBC in each of these model runs.

\subsubsection{Apparent interference from the southern sponge}

In the averages from model years 8 to 13 , we observe behavior that is inconsistent with an isolated interaction between the NRG and a bathymetric slope. In this subsection, we present some evidence that these inconsistencies are due to interactions with the southern sponge layer, which occupies the southern $800 \mathrm{~km}$ of the domain. To gauge the effect of the southern sponge, we consider the relative sizes of meridional eddy PV fluxes within the southern sponge in our model parameter variation experiments for two model time periods: years 3-8 and 8-13. The meridional eddy PV fluxes are integrated between $x_{w}$ and $x_{e}$, the zonal extent of the budget domain throughout this work. We also refer to the time series of domain integrated enstrophy and $\psi$ variance as they are a measure of the temporal dependence of model variability. Unfortunately, due to a server maintenance issue, enstrophy and $\psi$ variance fields were only output until year 11.4. As we will show, there are already indications of diverging behavior at this point. Time means are for the full year 8 through 13 range.

In the basic set of model runs, in which the bathymetric slope and DWBC are sequentially added, we observe no significant differences in the role of the southern sponge between model runs (see Figure 4-26). In the model runs with a slope, there is more eddy PV flux in the southern sponge in the time mean from years 8 to 13 than in the model run without a slope, but this is not as clear as the differences observed in the model parameter variations. 
When the inflowing velocity is varied and hence the jet stability, the more unstable runs are significantly more impacted by the southern sponge in the final 5 years of model time, likely because of their large meridional extent (see Figure 4-27). While from model years 3 to 8 the magnitude of eddy PV flux at the base of the slope and at the southern sponge increases monotonically with instability, from model years 8 to 13 , the eddy PV fluxes in model configurations with inflowing velocity greater or equal to $1.17 \mathrm{~m} \mathrm{~s}^{-1}$, whose recirculation gyres have larger natural recirculation gyre meridional extents, have increased, while the eddy PV fluxes of more stable runs, with smaller recirculation gyres, have decreased. This is accompanied by a reduction in $\psi$ variance in the most unstable configurations, while $\psi$ variance continues to increase in the more stable configurations, as highlighted in Figure 4-28. This difference is not as apparent in Figure 4-27 because of the large differences in total domain integrated fields between model runs.

This split in behavior as the model continues to spin-up is most apparent when the distance between the unstable jet and the slope is varied (see Figure 4-29). The $\psi$ variance of model runs with $400 \mathrm{~km}$ or more between the jet and the slope begins to fall at about year 8 , while in model runs with the jet and slope closer together it continues to rise gradually. This split is also evident in the evolution of the meridional eddy PV fluxes. From model years 3 to 8 , there was a continuous decrease in meridional eddy PV flux as the distance between the jet and the slope decreased, including a suppression of eddy PV fluxes at the jet axis. As noted in the main text, the model run with $450 \mathrm{~km}$ between the jet and the slope does not follow this pattern. From model years 8 to 13 the same model runs that had a large drop in psi variance showed an increase in magnitude of eddy PV flux at the southern sponge and at the base of the slope. The model runs with less distance between the jet and the slope have decreased eddy PV fluxes at the edges of the recirculation gyres, consistent with the convergence to steady state that is suggested by their $\psi$ variance and enstrophy time series. As seen when varying the model instability, this difference in evolving behavior is likely caused by the difference in proximity to the southern sponge layer. These spurious model evolutions point to the importance of considering the distance between the recirculation gyre and southern sponge in developing this work further. 

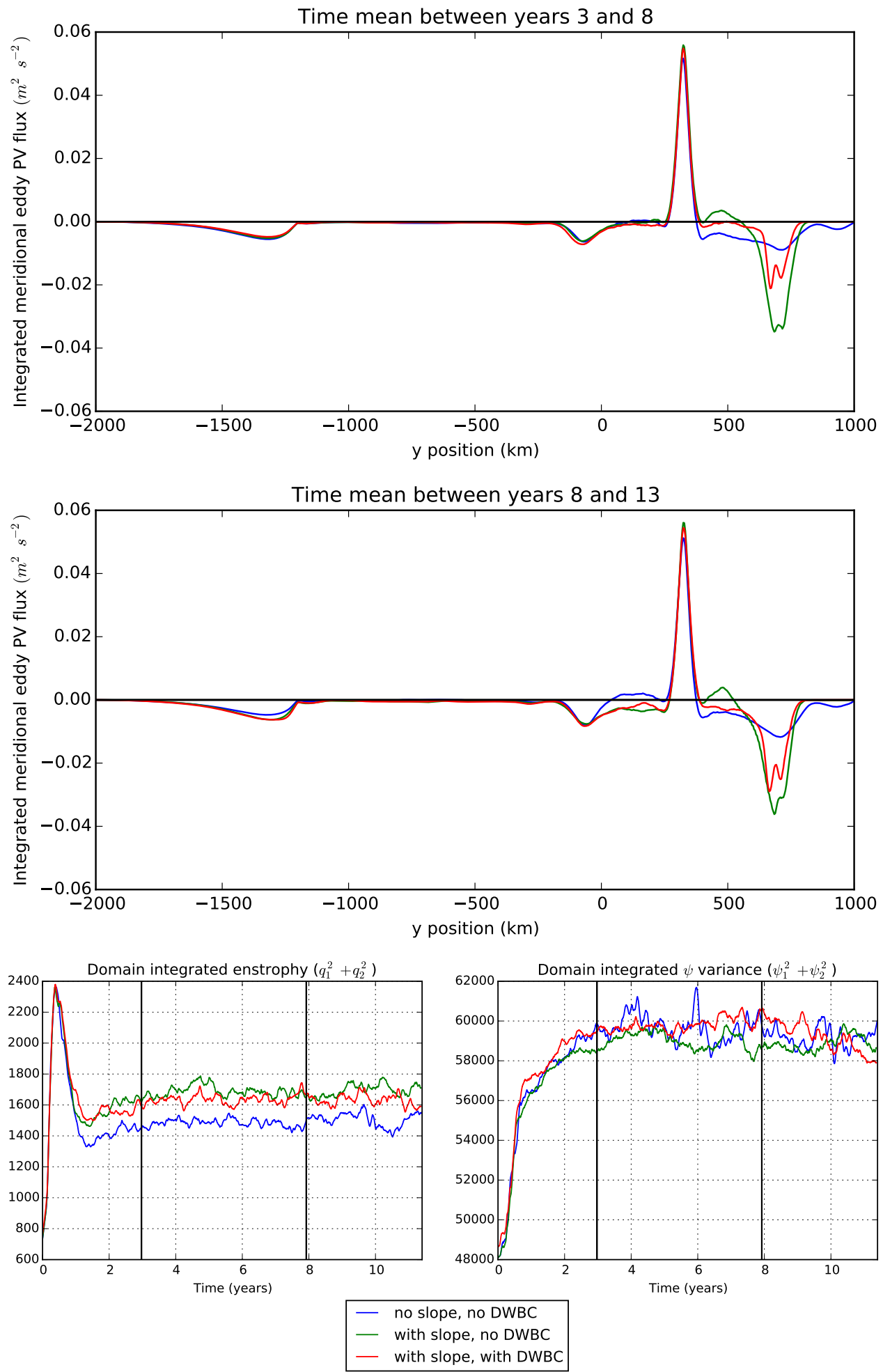

Figure 4-26: Top: Meridional profiles of meridional eddy PV flux, integrated from $x_{w}$ to $x_{e}$, i.e. $\int_{x_{e}}^{x_{w}} \overline{v^{\prime} q^{\prime}} d x$ for the time mean between model years 3 and 8. Center: Meridional profiles of meridional eddy PV flux for the time mean between model years 8 and 13 . Bottom left: Domain integrated enstrophy, $q_{1}^{2}+q_{2}^{2}$ (non-dimensional). Bottom right: Domain integrated $\psi$ variance, $\psi_{1}^{2}+\psi_{2}^{2}$ (non-dimensional). In each case properties for model runs without a slope or DWBC (blue), with a slope, but no DWBC (green), and with both a slope and DWBC (red) are shown. The inflowing jet velocity is $1.17 \mathrm{~m} \mathrm{~s}^{-1}$, and there is $400 \mathrm{~km}$ between the center of the jet and the slope when the slope is present. 
Time mean between years 3 and 8

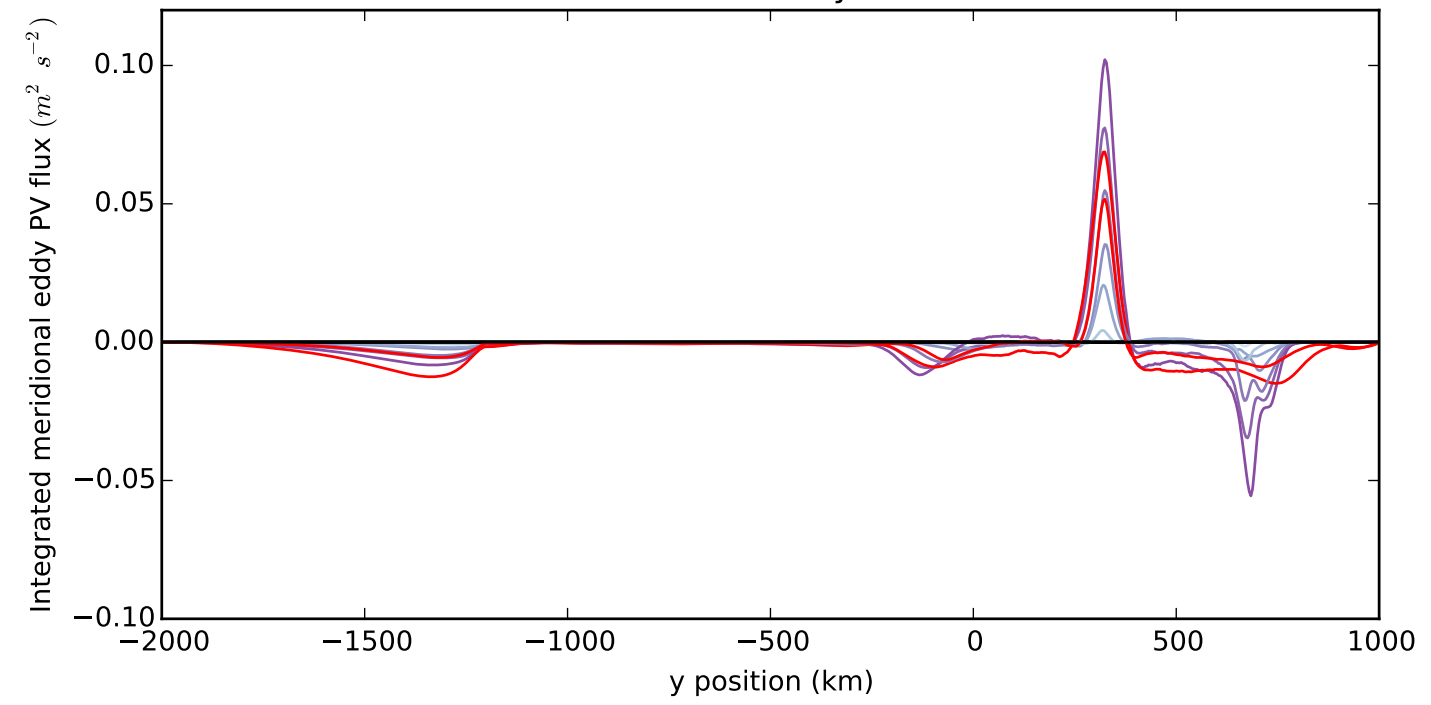

Time mean between years 8 and 13
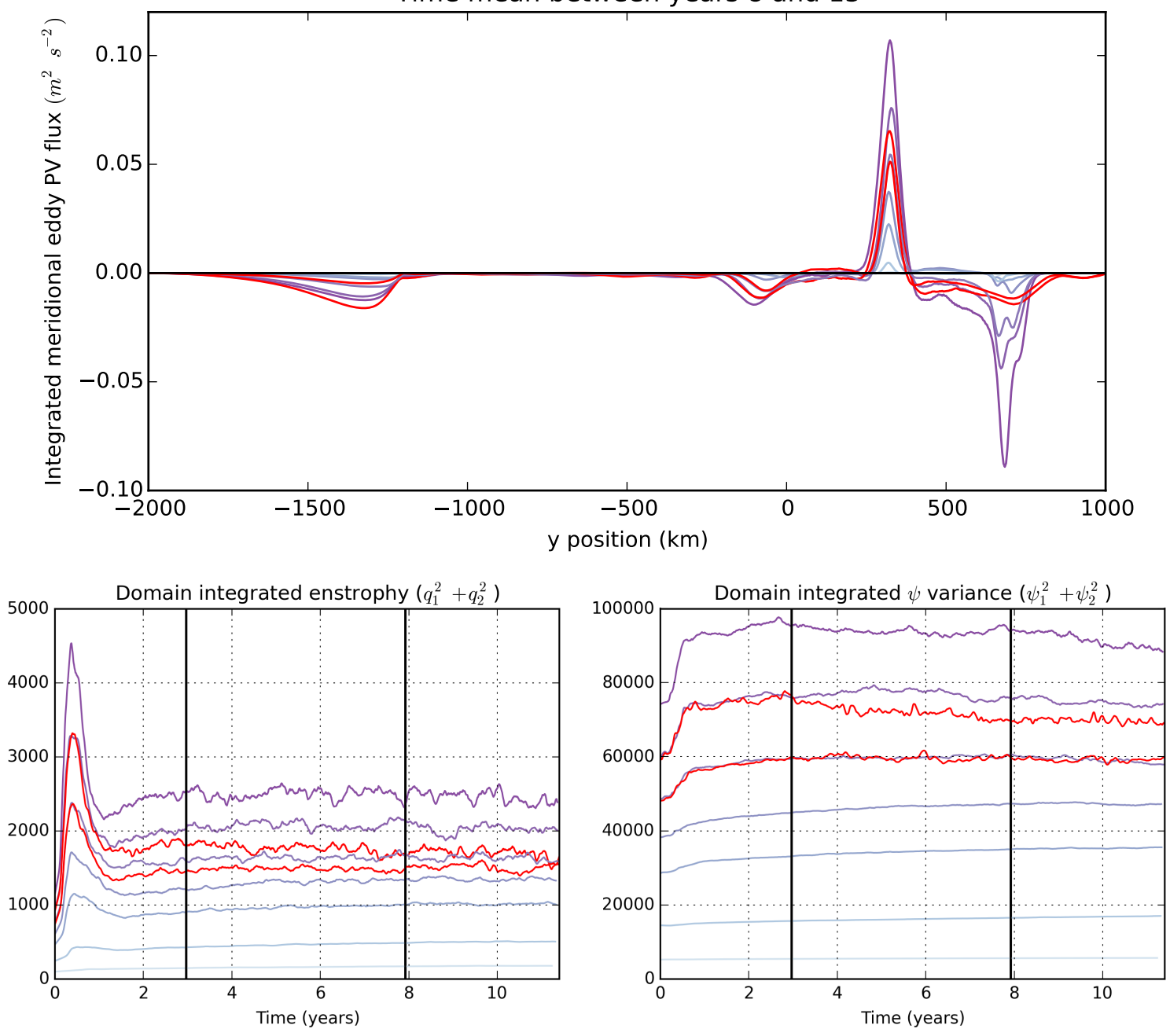

Figure 4-27: As in Figure 4-26, but for model configurations with different inflowing jet velocities. Color coding is as in Section 4.7.1; the range of velocities is 0.36 to $1.44 \mathrm{~m} \mathrm{~s}^{-1}$, from light to dark. Profiles in red has no slope or DWBC in the lower layer, and has inflowing jet velocity of 1.17 and $1.3 \mathrm{~ms}^{-1}$. 

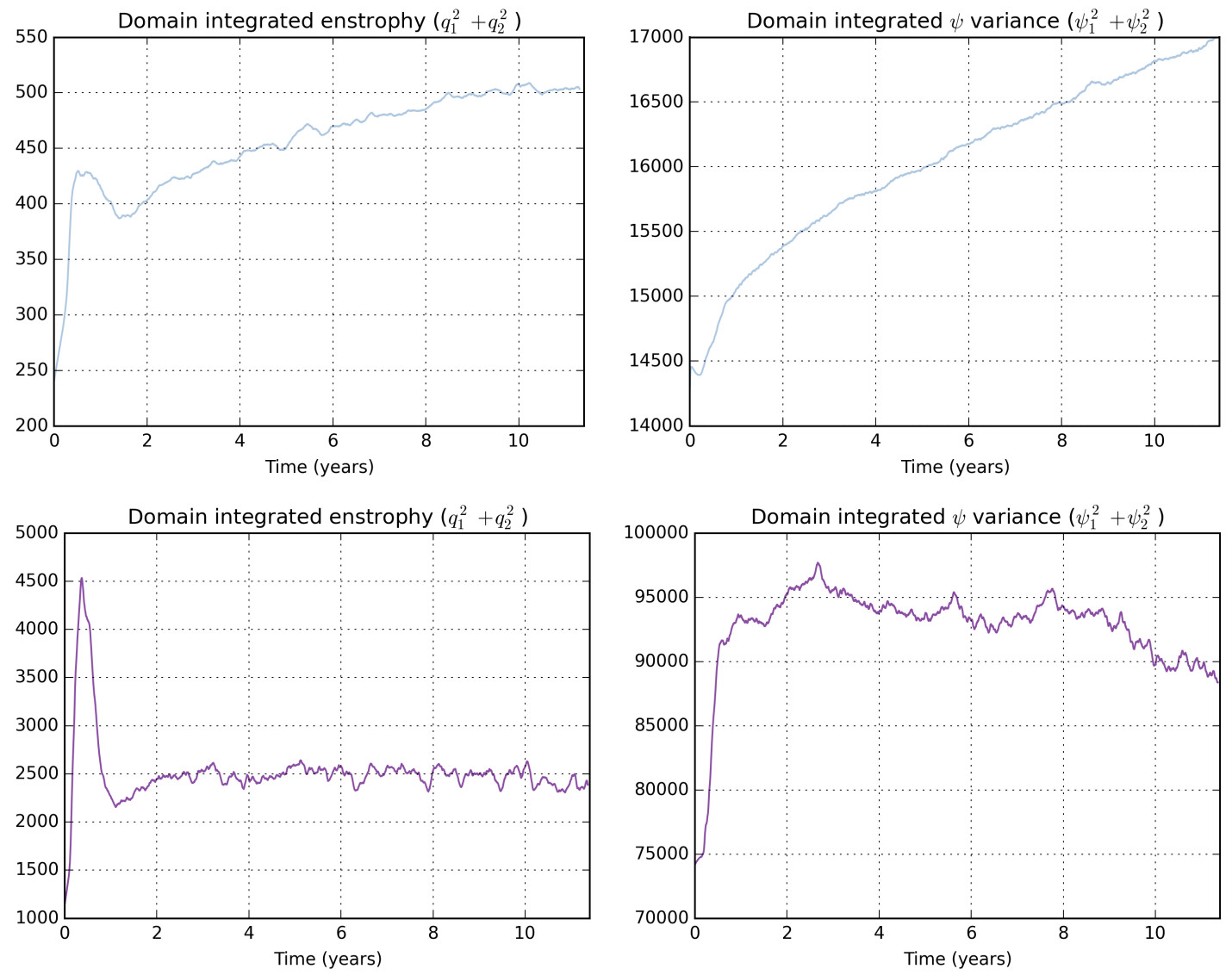

Figure 4-28: Time series of domain integrated enstrophy and $\psi$ for model configurations with inflowing jet velocity of $0.63 \mathrm{~m} \mathrm{~s}^{-1}$ (top panel) and $1.44 \mathrm{~m} \mathrm{~s}^{-1}$ (bottom panel). 
Time mean between years 3 and 8
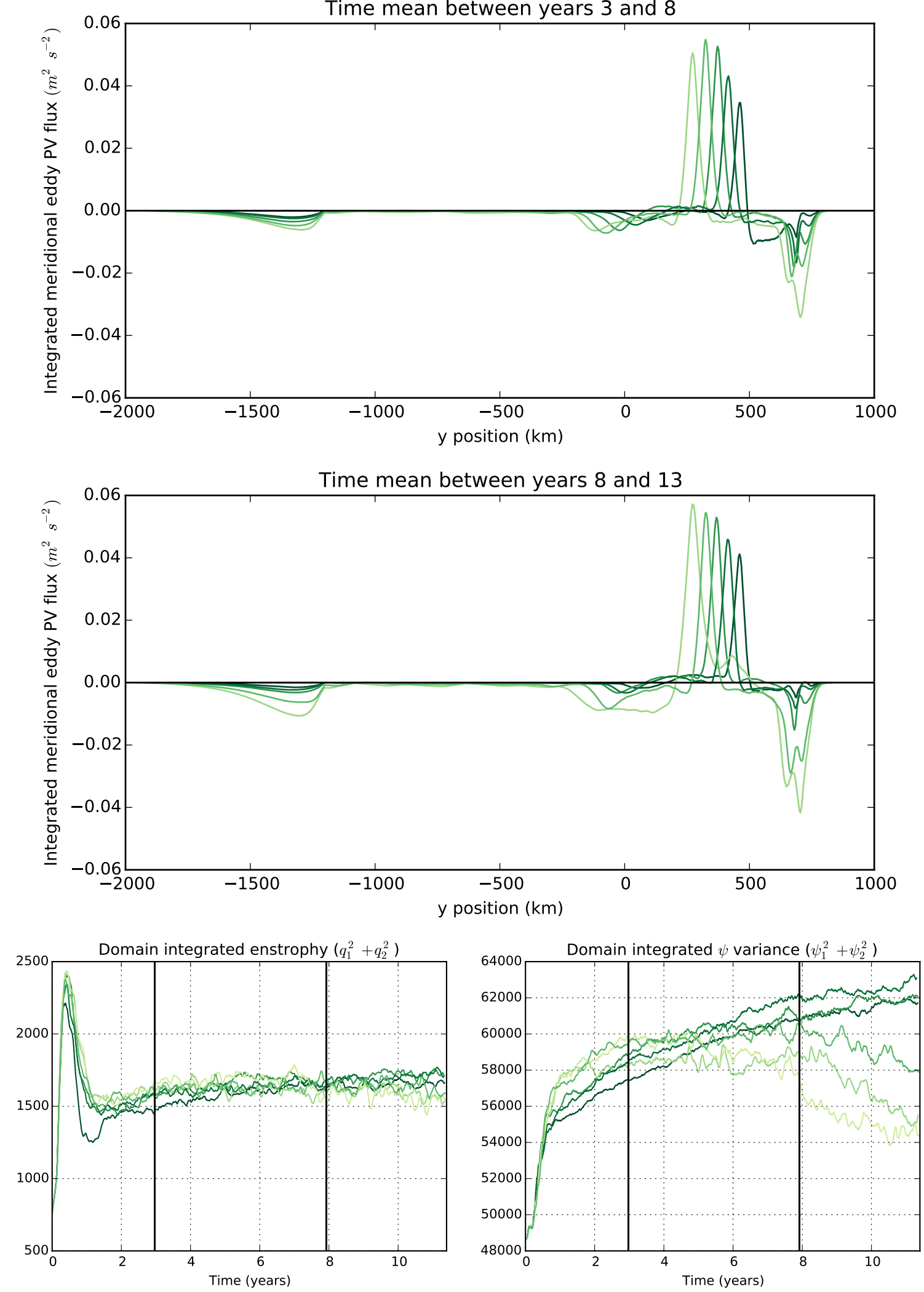

Figure 4-29: As in Figure 4-26, but for model configurations with different distances between the jet and the center of the bathymetric slope. Color coding is as in Section 4.7.2; the range of distances is from 250 to $450 \mathrm{~km}$, from dark to light. 


\subsubsection{Estimating the time rate of change of $q_{2}$}

Though we were unable to calculate the mean time rate of change of $q_{2}$ in the model directly, we can make some estimates of the changes in mean $q_{2}$ field to provide context and check whether the residuals in our PV budgets are reasonable. In Figures 4-30, 4-31 and 4-32, we show how the residuals from the PV budget (in black) compare to the mean change in $q_{2}$ from the initial model conditions (in red) and from an earlier model mean (in orange).

In general, $q_{2}$ shows an increase in the NRG region and decrease in the DWBC region from the initial conditions in a manner that is consistent with the changes inferred from the PV budget residuals, i.e. a larger change for more unstable model configurations (see Figure 4-31) and a smaller change when the jet is brought closer to the slope (see Figure 4-32). A notable exception to this consistency is the set of runs in which a slope and DWBC are added sequentially (Figure 4-30). The rate of change between the year 0-3 mean and year 8-13 mean is noisier as it highlights differences in the way model configurations spin up.

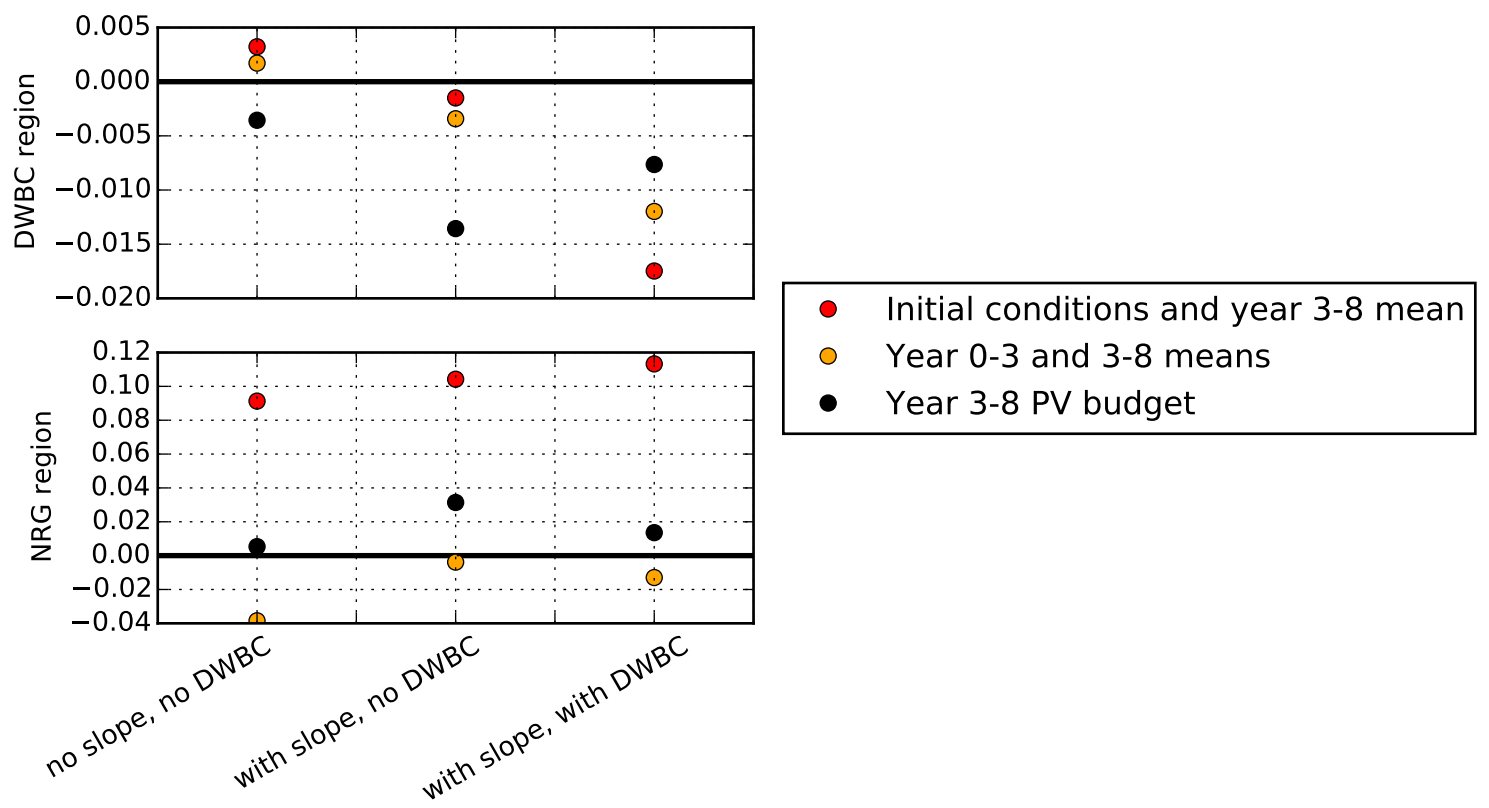

Figure 4-30: Estimates of $\int\left(\overline{d q_{2} / d t}\right) d A$ in the NRG and DWBC regions for base case runs in which a slope and DWBC are added sequentially. In red we show $\left(\overline{q_{2}}-q_{2 i n i t}\right) / 5.5$ years, and in orange $\left(\overline{q_{2}}-\hat{q_{2}}\right) / 4$ years, where $q_{2 i n i t}$ is the initial $q_{2}, \overline{q_{2}}$ is the time mean for model years $3-8$ and $\hat{q_{2}}$ is the time mean for model years $0-3$. We show the residual from the PV budgets presented in the main text for model years 3-8 in black for reference. 


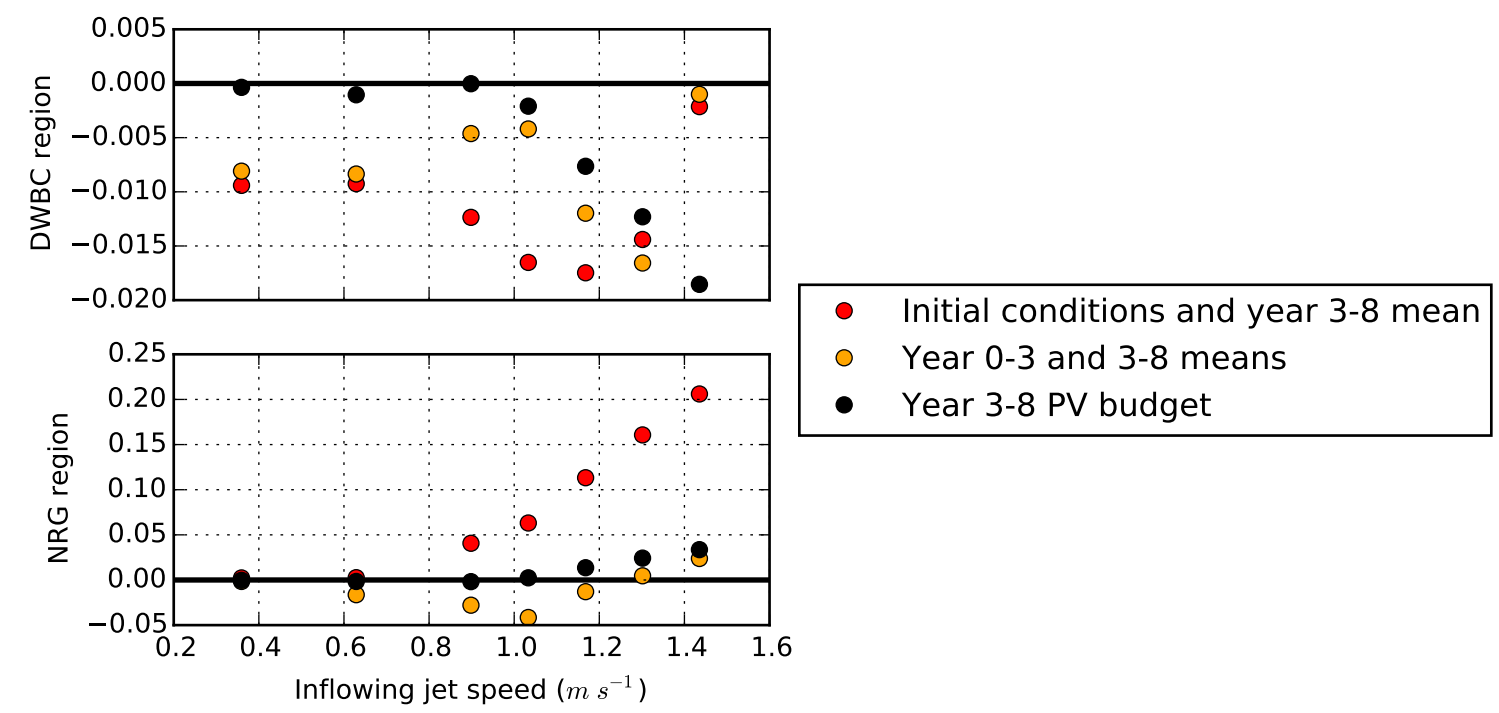

Figure 4-31: As in Figure 4-30 but for model configurations with different inflowing jet velocities.
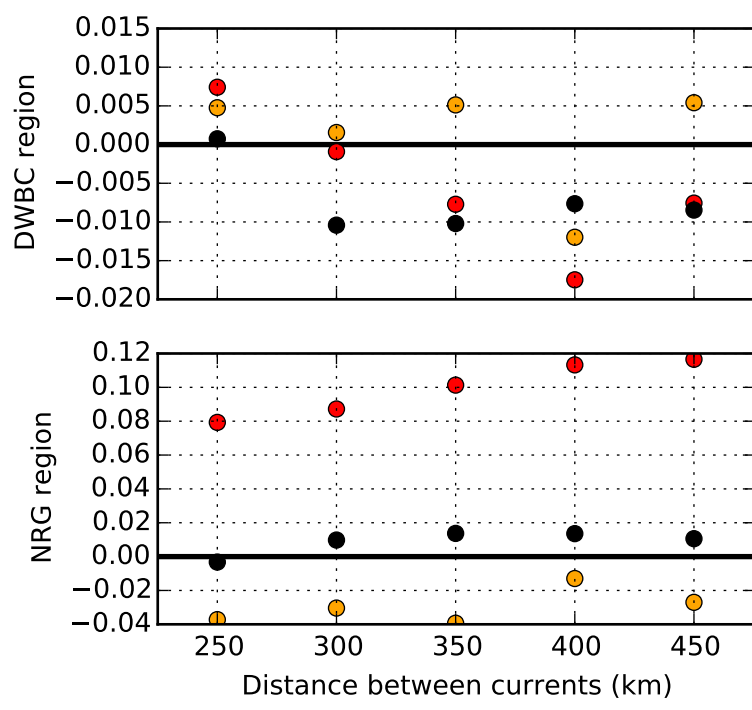
- Initial conditions and year 3-8 mean - Year 0-3 and 3-8 means
- Year 3-8 PV budget

Figure 4-32: As in Figure 4-30 but for model configurations with different distances between the jet and the center of the bathymetric slope. 
Chapter 5

Conclusions 
This thesis investigates the dynamics of two western boundary current systems in the North Atlantic, the Gulf Stream and the Deep Western Boundary Current (DWBC), from a combination of observations and models. This includes Chapter 2, devoted to the forcing mechanisms of the Gulf Stream; Chapter 3, focused on the propagation of water mass properties in the DWBC and Chapter 4, a model study describing the interaction between the Gulf Stream and DWBC. Though disparate in their approaches, each chapter addresses questions relating to the fundamental structure of the large-scale circulation. In this conclusion chapter, I will review the primary contributions of each thesis chapter and outline the general implications of this work, as well as potential future research directions.

\subsection{Contributions}

In Chapter 2, we derive and diagnose the size of terms in a vorticity budget for a surface layer of the western North Atlantic, with a focus on observations. The major contributions of Chapter 2 include:

1. Clarification of the connection between budgets for depth-averaged and depth-integrated vorticity budgets.

2. A way to place inertial effects in context of forcing and dissipation in a budget for depth-averaged vorticity.

3. Demonstration that the primary balance of vorticity is between wind stress forcing and a source of vorticity in the western North Atlantic, such as friction, in observations and a state estimate.

Overall, we found that the Stommel (1948) and Munk (1950) frameworks remain relevant to our understanding of western boundary current dynamics, as the primary balance of the vorticity is between wind stress forcing and friction in ECCO state estimate. At the same time, we showed that the Gulf Stream has a significant inertial component in this framework, in keeping with developments in western boundary current theory (Fofonoff, 1954; Charney, 1955; Cessi, 1990; Waterman and Jayne, 2011). In general, Chapter 2 offers an updated interpretation of Gulf Stream dynamics as they relate to classical theories for a barotropic, rectangular ocean with a flat seafloor.

Chapter 3 is a study of the along-path evolution of water mass properties in the DWBC. Our focus is on DWBC observations at Line W, which is at $39^{\circ} \mathrm{N}$ on the continental slope southeast of New England, and on placing these observations in the context of DWBC observations upstream. The primary findings of Chapter 3 are: 
1. A statistically significant change in deep Labrador Sea Water properties is observed at Line $\mathrm{W}$ moorings consistent with prior changes at its formation site in the Labrador Sea.

2. Coherent patterns of change along the path of the DWBC are documented, with some indication that most of the water mass property changes between the Labrador Sea and Line $\mathrm{W}$ occur in the Tail of the Grand Banks region.

3. Our observations are consistent with an advective-diffusive model. We find realistic mean travel times between the Labrador Sea and Line W of 4-6 years and stirring time scales for exchange between the DWBC and the interior of 2-5 years.

This work shows that water mass property changes can be detected along the DWBC when changes are large enough at the source and that this coherence can be used to quantify bulk properties of the DWBC. Our observations are extending just long enough now to make this kind of calculation based on decadal scale changes. Importantly, Chapter 3 weighs in on an ongoing debate over the degree of meridional connectivity of the DWBC (Rhein et al., 2015), much of which has been focused on model results (Gary et al., 2011; Zou and Lozier, 2016). The findings in Chapter 3 highlight that both advective and diffusive processes are significant in the DWBC.

In Chapter 4, we focused on the interaction between the Gulf Stream's northern recirculation gyre and the DWBC, using an idealized two-layer quasi-geostrophic model. This led to a number of insights, namely:

1. A description of the recirculation gyre adjustment to a bathymetric slope in the lower layer of the model: proximity to the slope can constrain the meridional extent of the recirculation gyre, damp eddy PV fluxes from the unstable jet, and lengthen the recirculation gyre in the along-isobath direction.

2. An appreciation for the potential effects of this adjustment on the DWBC, which travels along the slope in the lower layer: the recirculation gyre can homogenize PV into the slope and merge with the DWBC. The eddy PV fluxes associated with the gyre's adjustment can stir DWBC and recirculation gyre properties.

This model provides a framework for thinking about the interaction between the northern recirculation gyre and DWBC. We hope to connect this framework with realistic models and observations in the future. 


\subsection{Implications and Outlook}

Each of the chapters deals with a different fundamental question relating the large-scale circulation, but all fit within the same context. In this section, I will summarize the current understanding of the overturning circulation in the North Atlantic and how this thesis compliments and challenges this paradigm.

The Atlantic Meridional Overturning Circulation (AMOC), is frequently conceptualized as a conveyor belt. As a result, many studies describe the AMOC using one quantity: the zonal integral of the poleward upper-ocean mass transport across the basin, which is compensated by an equivalent zonally integrated equatorward flow at depth. In reality, this number is a combination of many physical processes, and varies significantly with latitude. Its utility as a measure of the ocean's role in climate is questionable (Lozier, 2010; Wunsch and Heimbach, 2013b), and in this thesis we have instead focused two of the components of the AMOC, rather than the system as a whole.

Despite its limitations, this characterization of the AMOC has been the focus of the climate modeling community. Most climate models predict that the overturning circulation will slow in response to global climate change, as reported in the 2014 IPCC report (Pachauri and Meyer, 2014). In these models, warming air temperatures and increasing freshwater input from melting ice caps both suppress deep water formation, leading to a slowing of the overturning circulation.

While there is historical evidence from the "Great Salinity Anomaly" of the 1970's that large inputs of freshwater can halt deep water formation (Dickson et al., 1996), the link between deep water formation and the strength of the overturning circulation remains unclear (Lozier, 2012). In fact, observations indicate that changes in deep water formation have no impact on the strength of the lower limb of the overturning circulation: the DWBC. For example, measurements of the DWBC at $53^{\circ} \mathrm{N}$ show that the DWBC transport exiting the Labrador Sea remained statistically constant between 1997 and 2009 despite large changes in the rate of Labrador Sea Water formation (Fischer et al., 2010). Similar conclusions were reached by Schott et al. (2006) from measurements of the DWBC east of the Grand Banks and Toole et al. (2011) from DWBC measurements at Line W.

However, recent observations and modeling work imply that the lower limb of the overturning circulation is not solely focused on the western boundary of the North Atlantic basin, but also travels equatorward in a broad swath of the subtropical North Atlantic, or an interior pathway (Bower et al., 2009; Gary et al., 2011). This implies that arrays focused on the DWBC are not measuring the full equatorward transport of the overturning circulation's lower limb, particularly at Line W.

At the same time, in Chapter 3, we showed that changes in water mass properties measured in the DWBC at Line $\mathrm{W}$ are consistent with changes in water mass properties 
at their source in the Labrador Sea. This implies some transfer of information between the subpolar and subtropical North Atlantic along the western boundary, which could have feedbacks on the overturning circulation. For example, Jackson et al. (2016) hypothesize that changing densities of LSW can result in changes in across-basin geostrophic transport as these density anomalies are advected equatorward, and use this mechanism to explain the decrease in AMOC measured by the across-basin Rapid array at $24^{\circ} \mathrm{N}$ from 2004 to 2014 . The MOVE array at $16^{\circ} \mathrm{N}$ measures the AMOC west of the mid-Atlantic ridge and report coherence in transport variability with the Rapid array (Send et al., 2011).

Especially as oceanographic data sets such as Line W, Rapid and the MOVE array pass the ten year mark, we need a better understanding of the different dynamical processes that shape the circulation of the North Atlantic on decadal timescales. This is particularly relevant in light of the predictions that the overturning circulation will slow over the next centuries; an understanding of the processes involved will help us distinguish between decadal variability and long term trends (Srokosz and Bryden, 2015). An understanding of the distribution of the equatorward transport at depth is a critical first step to understanding the processes that may be significant. In Chapter 3, we made bulk estimates of the advection and stirring which shape the distribution of the equatorward transport, shedding light on the spreading of deep water in the North Atlantic.

In Chapter 4, we investigated one dynamical process that could lead to the stirring we quantified in Chapter 3, namely the adjustment of the Gulf Stream's recirculation gyre to the continental slope. By isolating this process in a model, we showed that return flow of the northern recirculation gyre may be indistinguishable from the DWBC at depth, and that their interaction could induce PV and tracer fluxes between the DWBC and the northern recirculation gyre. One way to bring our findings in Chapter 3 and 4 together would be to calculate eddy PV fluxes in the DWBC from Line W moored measurements. Although Line $\mathrm{W}$ is at the southern end of the northern recirculation gyre, this could shed light on the relevance of the eddy PV fluxes we modeled.

Further, in our model, the majority of the DWBC transport change due to the interaction between the northern recirculation gyre and the DWBC occurs all at once as the DWBC reaches the northern recirculation gyre. In reality, this point is at the Tail of the Grand Banks, and this mechanism could explain the heightened exchange between the DWBC and the interior at the Tail of the Grand Banks inferred from float observations (Bower et al., 2009). An interesting extension to our model would be a "Tail of the Grand Banks" feature that could close the mean PV contours of the northern recirculation gyre and better mimic this transition.

The model in Chapter 4 demonstrates that the coupling between the upper and deep ocean can shape the circulation at depth. However, this simplified process model does not consider feedbacks between the Gulf Stream and DWBC, which could lead to natural decadal 
variability in the AMOC (Spall, 1996b; Katsman et al., 2001). In Chapter 2 we focus on the dynamics of the wind-driven circulation in isolation, but one of our primary contributions is a framework for understanding the vorticity balance of the depth-averaged circulation. We applied this framework to observations and a coarse-resolution state estimate. It would be interesting to extend this application to higher resolution models in order to elucidate the eddy processes which are parameterized as friction and diffusion in the low resolution state estimate. Another interesting extension would be to consider the vorticity balance of the deep circulation which was the focus of Chapters 3 and 4 , and how the vorticity balances of the surface and deep circulations fit together in the full overturning circulation.

Studying the role of the large-scale ocean circulation in climate necessitates longer and more complete datasets, especially in the deep ocean. Unfortunately, as pointed out in Wunsch and Heimbach (2013b), modern observations can only increase in duration at a rate

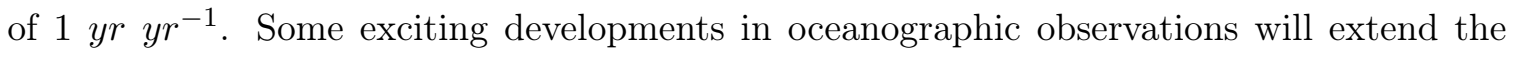
coverage of global observations to the deep ocean: the deep Argo program and deep glider. Yet it will likely be at least another decade before these data have global coverage, and so shipboard and moored observations of the deep ocean will remain critically important.

The Rapid array was the first moored array to measure the cross-basin overturning circulation. It has recently been complemented by an array across the subpolar North Atlantic (OSNAP, Overturning in the Subpolar North Atlantic Project) which will shed light on the relationship between variability in deep water mass properties and overturning circulation strength. The OSNAP measurements come at an exciting time as convection in the Labrador Sea has been deepening since 2012, forming a fresh and cold anomaly at depth similar in strength to the event in the mid 1990s we focus on in Chapter 3 (Igor Yashayaev, personal communication). Though there are no concrete plans for an extension of the Line W program, our hope is that opportunistic measurements in the region will allow us to test the deep Labrador Sea water transit time distributions we calculated in Chapter 3, as this new anomaly makes its way equatorward.

This thesis has explored multiple facets of the North Atlantic circulation, through a combination of models and observations. While models are useful in building a more complete understanding of the dynamics, observations are critical in assessing their relevance. Moving forward I hope to remain grounded in observations, and continue to work towards piecing together the complex structure of the global ocean circulation. 


\section{BIBLIOGRAPHY}

Adams, J., Swartztrauber, P., and Sweet, R. FISHPAK: A package of FORTRAN subprograms for the solution of separable elliptic partial differential equations. Version 3.2. 1988.

Andres, M., Toole, J. M., Torres, D. J., Smethie, W. M., Joyce, T. M., and Curry, R. G. Stirring by deep cyclones and the evolution of Denmark strait overflow water observed at line W. Deep Sea Research I, 109:10-26, 2015.

Arakawa, A. Computational design for long-term numerical integration of the equations of fluid motion: Two-dimensional incompressible flow. Part 1. Journal of Computational Physics, 1:119-143, 1966.

Berloff, P., Hogg, A. M., and Dewar, W. The turbulent oscillator: A mechanism of lowfrequency variability of the wind-driven ocean gyres. Journal of Physical Oceanography, $37: 2363-2386,2007$.

Bower, A. S. and Hogg, N. G. Structure of the Gulf Stream and its recirculations at $55^{\circ} \mathrm{W}$. Journal of Physical Oceanography, 26:1002-1022, 1996.

Bower, A. S. and Hogg, N. G. Low-frequency variability on the western flanks of the Grand Banks. Journal of Marine Research, 58:523-545, 2000.

Bower, A. S. and Hunt, H. D. Lagrangian observations of the Deep Western Boundary Current in the North Atlantic ocean. Part I: Large-scale pathways and spreading rates. Journal of Physical Oceanography, 30:764-783, 2000.

Bower, A. S., Rossby, H. T., and Lillibridge, J. L. The Gulf Stream - barrier or blender? Journal of Physical Oceanography, 15:24-32, 1985.

Bower, A. S., Lozier, M. S., Gary, S. F., and Böning, C. W. Interior pathways of the North Atlantic meridional overturning circulation. Nature, 459:243-247, 2009.

Bower, A. S. and Hogg, N. G. Evidence for barotropic wave radiation for the Gulf Stream. Journal of Physical Oceanography, 22:42-61, 1992.

Bower, A. S., Lozier, S., Gary, S. F., Lozier, M. S., and Gary, S. F. Export of Labrador Sea Water from the subpolar North Atlantic: A Lagrangian perspective. Deep Sea Research II, 58:1798-1818, 2011.

Bower, A. S., Hendry, R. M., Amrhein, D. E., and Lilly, J. M. Direct observations of formation and propagation of subpolar eddies into the Subtropical North Atlantic. Deep Sea Research II, 85:15-41, 2013.

Buckley, M. W. and Marshall, J. Observations, inferences, and mechanisms of the Atlantic Meridional Overturning Circulation: A review. Reviews of Geophysics, 54:1-59, 2015. 
Cane, M. A., Kamenkovich, V. M., and Krupitsky, A. On the utility and disutility of JEBAR. Journal of Physical Oceanography, 28:519-526, 1998.

Cessi, P. Recirculation and separation of boundary currents. Journal of Marine Research, 48:1-35, 1990.

Charney, J. G. The Gulf Stream as an inertial boundary layer. Proceedings of the National Academy of Sciences of the United States of America, 41:731-740, 1955.

Charney, J. G. and Flierl, G. R. Oceanic analogues of large-scale atmospheric motions. In Evolution of Physical Oceanography, Scientific Surveys in Honor of Henry Stommel, pages 504-548. MIT Press, Association for the Sciences of Limnology and Oceanography, Inc, 1981.

Cherian, D. A. When an eddy encounters shelf-slope topography. PhD thesis, 2016.

Curry, R. G., McCartney, M. S., and Joyce, T. M. Oceanic transport of subpolar climate signals to mid-depth subtropical waters. Nature, 391:575-577, 1998.

Czaja, A. and Hausmann, U. Observations of entry and exit of potential vorticity at the sea surface. Journal of Physical Oceanography, 39:2280-2294, 2009.

Deacon, G. E. R. Exploration of the deep sea. Journal of Navigation, 7:165 - 174, 1954.

Dee, D. P., Uppala, S. M., Simmons, a. J., Berrisford, P., Poli, P., Kobayashi, S., Andrae, U., Balmaseda, M. a., Balsamo, G., Bauer, P., Bechtold, P., Beljaars, a. C. M., van de Berg, L., Bidlot, J., Bormann, N., Delsol, C., Dragani, R., Fuentes, M., Geer, a. J., Haimberger, L., Healy, S. B., Hersbach, H., Hólm, E. V., Isaksen, L., Kållberg, P., Köhler, M., Matricardi, M., McNally, a. P., Monge-Sanz, B. M., Morcrette, J.-J., Park, B.-K., Peubey, C., de Rosnay, P., Tavolato, C., Thépaut, J.-N., and Vitart, F. The ERA-Interim reanalysis: configuration and performance of the data assimilation system. Quarterly Journal of the Royal Meteorological Society, 137:553-597, 2011.

Dengler, M., Schott, F. A., Eden, C., Brandt, P., Fischer, J., and Zantopp, R. J. Break-up of the Atlantic deep western boundary current into eddies at $8^{\circ} \mathrm{S}$. Nature, 432:1018-1020, 2004 .

Dengler, M., Fischer, J., Schott, F. A., and Zantopp, R. Deep Labrador Current and its variability in 1996âĂŞ2005. Geophysical Research Letters, 33:L21S06, 2006.

Deremble, B., Wienders, N., and Dewar, W. K. Potential vorticity budgets in the North Atlantic ocean. Journal of Physical Oceanography, 44:164-178, 2014.

Dickson, B., Yashayaev, I., Meincke, J., Turrell, B., Dye, S., Holfort, J., Dickson, R. R., and Brown, J. Rapid freshening of the deep North Atlantic Ocean over the past four decades. Nature, 99:832-837, 2002.

Dickson, R., Lazier, J., Meincke, J., Rhines, P., and Swift, J. Long-term coordinated changes in the convective activity of the North Atlantic. Progress in Oceanography, 38:241-295, 1996.

Doney, S. C. and Jenkins, W. J. Ventilation of the deep western boundary current and abyssal western North Atlantic: Estimates from tritium and 3He distributions. Journal of Physical Oceanography, 24:638-659, 1994. 
Dong, S., Baringer, M. O., Goni, G. J., Meinen, C. S., and Garzoli, S. L. Seasonal variations in the South Atlantic Meridional Overturning Circulation from observations and numerical models. Geophysical Research Letters, 41:4611-4618, 2014.

Durran, D. R. The third-order Adams-Bashforth method: An attractive alternative to leapfrog time differencing. Monthly Weather Review, 119:702-720, 1991.

ECMWF. ERA-Interm Project, Monthly Means. Research Data Archive at the National Center for Atmospheric Research, Computational and Information Systems http://rda.ucar.edu/datasets/ds627.1, 2012.

Efron, B. and Gong, G. A leisurely look at the bootstrap, the jackknife, and cross-validation. The American Statistician, 37:36-48, 1983.

Ertel, H. Ein neuer hydrodynamischer Erhaltungssatz. Die Naturwissenschaften, 30:543$544,1942$.

Fischer, J., Karstensen, J., Zantopp, R., Visbeck, M., Biastoch, A., Behrens, E., Böning, C. W., Quadfasel, D., Jochumsen, K., Valdimarsson, H., Jónsson, S., Bacon, S., Holliday, N. P., Dye, S., Rhein, M., and Mertens, C. Intra-seasonal variability of the DWBC in the western subpolar North Atlantic. Progress in Oceanography, 132:233-249, 2015.

Fischer, J., Visbeck, M., Zantopp, R., and Nunes, N. Interannual to decadal variability of outflow from the Labrador Sea. Geophysical Research Letters, 37:L24610, 2010.

Flierl, G. Isolated eddy models in geophysics. Annual Review of Fluid Mechanics, 19: 493-530, 1987.

Fofonoff, N. Steady flow in a frictionless homogeneous ocean. Journal of Marine Research, 13:254-262, 1954.

Fox-Kemper, B. and Pedlosky, J. Wind-driven barotropic gyre I: Circulation control by eddy vorticity fluxes to an enhanced removal region. Journal of Marine Research, 62:169-193, 2004 .

Gary, S. F., Lozier, M. S., Biastoch, A., and Böning, C. W. Reconciling tracer and float observations of the export pathways of Labrador Sea Water. Geophysical Research Letters, 39:L24606, 2012.

Gary, S. F., Lozier, M. S., Claus, W. B., and Biastoch, A. Deciphering the pathways for the deep limb of the Meridional Overturning Circulation. Deep Sea Research II, 2011.

Gouretski, V. V. and Koltermann, K. P. WOCE Global Hydrographic Climatology A Technical Report. Technical report, Bundesamt fuer Seeschifffahrt und Hydrographie, 2004.

Gray, A. R. and Riser, S. C. A Global Analysis of Sverdrup Balance Using Absolute Geostrophic Velocities from Argo. Journal of Physical Oceanography, 44:1213-1229, 2014.

Greatbatch, R. J. A model for the inertial recirculation of a gyre. Journal of Marine Research, 45:601-634, 1987.

Hansen, B. and Osterhus, S. North Atlantic - Nordic Seas exchanges. Progress in Oceanography, 45:109-208, 2000. 
Hautala, S. L., Roemmich, D. H., and Schmitz, W. J. Is the North Pacific in Sverdrup balance along $24^{\circ} \mathrm{N}$ ? Journal of Geophysical Research, 99:41-52, 1994.

Haynes, P. H. and McIntyre, M. E. On the evolution of potential vorticity in the presence of diabatic heating and frictional or other forces. Journal of Atmospheric Sciences, 44: 828-841, 1987.

Hogg, N. G. A note on the deep circulation of the western North Atlantic: Its nature and causes. Deep Sea Research, 30, 1983.

Hogg, N. G. On the transport of the Gulf Stream between Cape Hatteras and the Grand Banks. Deep Sea Research, 39:1231-1246, 1992.

Hogg, N. G., Pickart, R. S., Hendry, R. M., and Smethie, W. J. The northern recirculation gyre of the Gulf Stream. Deep Sea Research, 33:1139-1165, 1986.

Hogg, N. and Stommel, H. On the relation between the deep circulation and the Gulf Stream. Deep Sea Research, 32:1181-1193, 1985.

Holland, W. R., Keffer, T., and Rhines, P. B. Dynamics of the oceanic general circulation: The potential vorticity field. Nature, 308:698-705, 1984.

Holland, W. R. Baroclinic and topographic influences on the transport in western boundary currents. Geophysical Fluid Dynamics, 4:187-210, 1972.

Holland, W. R. The role of mesoscale eddies in the general circulation of the ocean Numerical experiments using a wind-driven quasi-geostrophic model. Journal of Physical Oceanography, 8:363-392, 1978.

Holland, W. R. and Rhines, P. B. An example of eddy-induced ocean circulation. Journal of Physical Oceanography, 10:1010-1031, 1980.

Holte, J. and Talley, L. A new algorithm for finding mixed layer depths with applications to Argo data and subantarctic mode water formation. Journal of Atmospheric and Oceanic Technology, 26:1920-1939, 2009.

Hughes, C. W. and de Cuevas, B. A. Why western boundary currents in realistic oceans are inviscid: A link between form stress and bottom pressure torques. Journal of Physical Oceanography, 31:2871-2885, 2001.

Hummels, R., Brandt, P., Dengler, M., Fischer, J., Araujo, M., Veleda, D., and Durgadoo, $\mathrm{J}$. V. Interannual to decadal changes in the western boundary circulation in the Atlantic at $11^{\circ} \mathrm{S}$. Geophysical Research Letters, 42:7615-7622, 2015.

Hurrell, J. W. Decadal trends in the North Atlantic Oscillation: Regional temperatures and precipitation. Science, 269:676-9, 1995.

Hurrell, J. W. and Deser, C. North Atlantic climate variability: The role of the North Atlantic Oscillation. Journal of Marine Systems, 79:231-244, 2010.

Jackett, D. R. and McDougall, T. J. A neutral density variable for the world's oceans. Journal of Physical Oceanography, 27:237-263, 1997. 
Jackson, L., Hughes, C. W., and Williams, R. G. The role of bottom pressure torques and friction in basin and channel flows. Journal of Physical Oceanography, 36:1786-1805, 2006.

Jackson, L. C., Peterson, K. A., Roberts, C. D., and Wood, R. A. Recent slowing of Atlantic overturning circulation as a recovery from earlier strengthening. Nature Geoscience, pages $1-6,2016$.

Jayne, S. R. and Hogg, N. G. On recirculation forced by an unstable jet. Journal of Physical Oceanography, 29:2711-2718, 1999.

Jayne, S. R., Hogg, N. G., and Malanotte-Rizzoli, P. Recirculation gyres forced by a betaplane jet. Journal of Physical Oceanography, 26:492-504, 1996.

Joyce, T. M., Deser, C., and Spall, M. A. The relation between decadal variability of subtropical mode water and the North Atlantic Oscillation. Journal of Climate, 13:25502569, 2000.

Joyce, T. M., Dunworth-Baker, J., Pickart, R. S., Torres, D., and Waterman, S. On the Deep Western Boundary Current south of Cape Cod. Deep Sea Research II, 52:615-625, 2005 .

Kanzow, T., Send, U., and McCartney, M. On the variability of the deep meridional transports in the tropical North Atlantic. Deep Sea Research I, 55:1601-1623, 2008.

Kanzow, T., Send, U., Zenk, W., Chave, A. D., and Rhein, M. Monitoring the integrated deep meridional flow in the tropical North Atlantic: Long-term performance of a geostrophic array. Deep Sea Research I, 53:528-546, 2006.

Katsman, C. A., Drijfhout, S. S., and Dijkstra, H. A. The interaction of a deep western boundary current and the wind-driven gyres as a cause for low-frequency variability. Journal of Physical Oceanography, 31:2321-2339, 2001.

Kieke, D., Rhein, M., Stramma, L., Smethie, W. M., LeBel, D. A., and Zenk, W. Changes in the CFC inventories and formation rates of Upper Labrador Sea Water, 1997âĂ 2001. Journal of Physical Oceanography, 36:64-86, 2006.

Kieke, D., Klein, B., Stramma, L., Rhein, M., and Peter, K. Variability and propagation of Labrador Sea Water in the southern subpolar North Atlantic. Deep Sea Research I, 56: 1656-1674, 2009.

Knauss, J. A. A note on the transport of the Gulf Stream. Narraganset Marine Laboratory, University of Rhode Island, 1969.

Köhler, J., Mertens, C., Walter, M., Stöber, U., Rhein, M., and Kanzow, T. Variability in the internal wave field induced by the Atlantic Deep Western Boundary Current at $16^{\circ} \mathrm{N}$. Journal of Physical Oceanography, 44:492-516, 2014.

Kwon, Y. O., Alexander, M. A., Bond, N. A., Frankignoul, C., Nakamura, H., Qiu, B., and Thompson, L. Role of the Gulf Stream and Kuroshio-Oyashio systems in large-scale atmosphere-ocean interaction: A review. Journal of Climate, 23:3249-3281, 2010.

Lavender, K. L., Davis, R. E., and Owens, W. B. Mid-depth recirculation observed in the interior Labrador and Irminger seas by direct velocity measurements. Nature, 407, 2000. 
Lazier, J. R. N. Deep convection. In Ocean Circulation and Climate: Observing and Modelling the Global Ocean, pages 634-643. 2001.

Leaman, K., Johns, E., and Rossby, T. The average distribution of volume transport and potential vorticity with temperature at three sections across the Gulf Stream. Journal of Physical Oceanography, 19:36-51, 1989.

LeBel, D. A., Smethie, W. M., Rhein, M., Kieke, D., Fine, R. a., Bullister, J. L., Min, D. H., Roether, W., Weiss, R. F., Andrié, C., Smythe-Wright, D., and Peter Jones, E. The formation rate of North Atlantic Deep Water and Eighteen Degree Water calculated from CFC-11 inventories observed during WOCE. Deep Sea Research, 55:891-910, 2008.

Ledwell, J., Watson, A., and Law, C. Mixing of a tracer in the pycnocline. Journal of Geophysical Research: Oceans, 103:21,499-21,529, 1998.

Leetmaa, A., Niiler, P., and Stommel, H. Does the Sverdrup relation account for the midAtlantic circulation. Journal of Marine Research, 35:1-10, 1977.

Lozier, M. S. Evidence for large-scale eddy-driven gyres in the North Atlantic. Science, 277: 361-364, 1997.

Lozier, M. S. Deconstructing the conveyor belt. Science, 328:1507-11, 2010.

Lozier, M. S. Overturning in the North Atlantic. Annual Review of Marine Science, 4: 291-315, 2012.

Lozier, M. S., Gary, S. F., and Bower, A. S. Simulated pathways of the overflow waters in the North Atlantic: Subpolar to subtropical export. Deep Sea Research II, 85:147-153, 2013.

Lu, Y. and Stammer, D. Vorticity balance in coarse-resolution global ocean simulations. Journal of Physical Oceanography, 34:605-622, 2004.

Luyten, J. R., Pedlosky, J., and Stommel, H. The ventilated thermocline. Journal of Physical Oceanography, 13:292-309, 1983.

Lynn, R. J. and Reid, J. L. Characteristics and circulation of deep and abyssal waters. Deep Sea Research, 15:577-598, 1968.

MacLeish, W. H. The Gulf Stream. Houghton Mifflin Company, 1989.

Malanotte-Rizzoli, P., Hogg, N. G., and Young, R. E. Stochastic wave radiation by the Gulf Stream: Numerical experiments. Deep Sea Research I, 42:389-423, 1995.

Marshall, J. and Nurser, G. Steady, free circulation in a stratified quasi-geostrophic ocean. Journal of Physical Oceanography, 16:1799-1813, 1986.

Marshall, J., Jamous, D., and Nilsson, J. Entry, flux, and exit of potential vorticity in ocean circulation. Journal of Physical Oceanography, 31:777-789, 2001 .

Marshall, J., Johnson, H., and Goodman, J. A study of the interaction of the North Atlantic Oscillation with ocean circulation. Journal of Climate, 14:1399-1421, 2001b. 
McCartney, M. Recirculating components to the deep boundary current of the northern North Atlantic. Progress in Oceanography, 29:283-383, 1992.

McDowell, S., Rhines, P., and Keffer, T. North Atlantic potential vorticity and its relation to the general circulation. Journal of Physical Oceanography, 12:1417-1435, 1982.

Meinen, C. S. and Luther, D. S. Structure, transport, and vertical coherence of the Gulf Stream from the Straits of Florida to the Southeast Newfoundland Ridge. Deep Sea Research I, 112:137-154, 2016.

Meinen, C. S., Johns, W. E., Garzoli, S. L., van Sebille, E., Rayner, D., Kanzow, T., and Baringer, M. O. Variability of the Deep Western Boundary Current at $26.5^{\circ} \mathrm{N}$ during 2004-2009. Deep Sea Research II, 85:154-168, 2013a.

Meinen, C. S., Speich, S., Perez, R. C., Dong, S., Piola, A. R., Garzoli, S. L., Baringer, M. O., Gladyshev, S., and Campos, E. J. D. Temporal variability of the meridional overturning circulation at $34.5^{\circ} \mathrm{S}$ : Results from two pilot boundary arrays in the South Atlantic. Journal of Geophysical Research: Oceans, 118:6461-6478, $2013 \mathrm{~b}$.

Mertens, C., Rhein, M., Walter, M., Boning, C. W., Behrens, E., Kieke, D., Steinfeldt, R., and Stober, U. Circulation and transports in the Newfoundland Basin, western subpolar North Atlantic. Journal of Geophysical Research, pages 7772-7793, 2014.

Mertz, G. and Wright, D. G. Interpretations of the JEBAR Term. Journal of Physical Oceanography, 22:301-305, 1992.

Mizuta, G. Rossby wave radiation from an eastward jet and its recirculations. Journal of Marine Research, 67:185-212, 2009.

Molinari, R. L., Fine, R. a., Wilson, W. D., Curry, R. G., Abell, J., and McCartney, M. S. The arrival of recently formed Labrador sea water in the Deep Western Boundary Current at $26.5^{\circ}$ N. Geophysical Research Letters, 25:2249, 1998.

Munk, W. H. On the wind driven ocean circulation. Journal of Meteorology, 5:36-43, 1950.

Pachauri, R. and Meyer, L. Climate Change 2014: Synthesis Report. Contribution of Working Groups I, II and III to the Fifth Assessment Report of the Intergovernmental Panel on Climate Change (Core Writing Team). IPCC, Geneva, Switzerland, 2014.

Palter, J. B., Lozier, M. S., and Lavender, K. L. How does Labrador Sea Water enter the deep western boundary current? Journal of Physical Oceanography, 38:968-983, 2007.

Peña-Molino, B., Joyce, T. M., and Toole, J. M. Variability in the Deep Western Boundary Current: Local versus remote forcing. Journal of Geophysical Research, 117:C12022, 2012.

Peña-Molino, B., Joyce, T. M., and Toole, J. M. Recent changes in the Labrador Sea Water within the Deep Western Boundary Current southeast of Cape Cod. Deep Sea Research I, 58:1019-1030, 2010.

Pérez-Brunius, P., Rossby, T., and Watts, D. R. Absolute transports of mass and temperature for the North Atlantic Current-Subpolar Front system. Journal of Physical Oceanography, 34:1870-1883, 2004. 
Phillips, H. E. and Joyce, T. M. Bermuda's tale of two time series: Hydrostation S and BATS. Journal of Physical Oceanography, 37:554-571, 2007.

Pickart, R. S., Watts, D. R., Pickart, S., and Watts, D. R. Deep Western Boundary Current variability at Cape Hatteras. Journal of Marine Research, 48:765-791, 1990.

Pickart, R. S. and Smethie, W. M. J. How does the Deep Western Boundary Current cross the Gulf Stream? Journal of Physical Oceanography, 23:2602-2616, 1993.

Pickart, R. S., Hogg, N. G., and Smethie, W. M. Determining the strength of the Deep Western Boundary Current using the Chlorofluoromethane ratio. Journal of Physical Oceanography, 19:940-951, 1989.

Pickart, R. S., Spall, M. A., and Lazier, J. R. N. Mid-depth ventilation in the western boundary current system of the sub-polar gyre. Deep Sea Research I, 44:1025-1054, 1997.

Polton, J. A. and Marshall, D. P. Understanding the structure of the subtropical thermocline. Journal of Physical Oceanography, 33:1240-1249, 2003.

Rhein, M., Fischer, J., Smethie, W. M., Smythe-Wright, D., Weiss, R. F., Mertens, C., Min, D.-H., Fleischmann, U., and Putzka, A. Labrador Sea Water: Pathways, CFC inventory and formation rates. Journal of Physical Oceanography, 32:648-665, 2002.

Rhein, M. Drifters reveal deep circulation. Nature, 407:30-31, 2000.

Rhein, M., Kieke, D., and Steinfeldt, R. Ventilation of the upper Labrador Sea Water, 2003-2005. Geophysical Research Letters, 34:2003-2005, 2007.

Rhein, M., Kieke, D., Hüttl-Kabus, S., Roessler, A., Mertens, C., Meissner, R., Klein, B., Böning, C. W., and Yashayaev, I. Deep water formation, the subpolar gyre, and the meridional overturning circulation in the subpolar North Atlantic. Deep Sea Research II, 58:1819-1832, 2011.

Rhein, M., Kieke, D., and Steinfeldt, R. Advection of North Atlantic Deep Water from the Labrador Sea to the southern hemisphere. Journal of Geophysical Research: Oceans, 120, 2015 .

Rhines, P. B. and Holland, W. R. A theoretical discussion of eddy-driven mean flows. Dynamics of Atmospheres and Oceans, 3:289-325, 1979.

Rhines, P. B. and Young, W. R. Homogenization of potential vorticity in planetary gyres. Journal of Fluid Mechanics, 122:347-367, 1982.

Richardson, P. L. On the history of meridional overturning circulation schematic diagrams. Progress in Oceanography, 76:466-486, 2008.

Rienecker, M. M., Suarez, M. J., Gelaro, R., Todling, R., Bacmeister, J., Liu, E., Bosilovich, M. G., Schubert, S. D., Takacs, L., Kim, G.-K., Bloom, S., Chen, J., Collins, D., Conaty, A., da Silva, A., Gu, W., Joiner, J., Koster, R. D., Lucchesi, R., Molod, A., Owens, T., Pawson, S., Pegion, P., Redder, C. R., Reichle, R., Robertson, F. R., Ruddick, A. G., Sienkiewicz, M., and Woollen, J. MERRA: NASA's Modern-Era Retrospective Analysis for Research and Applications. Journal of Climate, 24:3624-3648, 2011. 
Risien, C. M. and Chelton, D. B. A Global Climatology of Surface Wind and Wind Stress Fields from Eight Years of QuikSCAT Scatterometer Data. Journal of Physical Oceanography, 38:2379-2413, 2008.

Rossby, C.-G. Dynamics of steady ocean currents the light of experimental fluid mechanics. Papers in Physical Oceanography and Meteorelogy, Published by Massachusetts Institute of Technology and Woods Hole Oceanographic Institution, V:Contribution No. 115, 1936.

Rossby, T. The North Atlantic Current and surrounding waters: At the crossroads. Reviews of Geophysics, 34:463, 1996.

Rossby, T. On gyre interactions. Deep Sea Research II, 46:139-164, 1999.

Rössler, A., Rhein, M., Kieke, D., and Mertens, C. Long-term observations of North Atlantic Current transport at the gateway between western and eastern Atlantic. Journal of Geophysical Research: Oceans, 120, 2015.

Rumford, C. Essay VII, The propagation of heat in fluids. Essays, Political, Economical, and Philosophical, A New Edition 2, London:197-386, 1800.

Sarkisyan, A. and Ivanov, V. Joint effect of baroclinicity and bottom relief as an important factor in the dynamics of sea currents. Izvestiya Akademii Nauk SSSR Fizika Atmosfery i Okeana, 7:173 - 188, 1971.

Saunders, P. M. The flux of overflow water through the Charlie-Gibbs Fracture Zone. Journal of Geophysical Research, 99:12343, 1994.

Schmittner, A. Decline of the marine ecosystem caused by a reduction in the Atlantic overturning circulation. Nature, 434:628-633, 2005.

Schmitz, W. J., Thompson, J. D., and Luyten, J. R. The Sverdrup Circulation for the Atlantic Along 24N. Journal of Geophysical Research, 97:7251-7256, 1992.

Schneider, L., Kieke, D., Jochumsen, K., Colbourne, E., Yashayaev, I., Steinfeldt, R., Varotsou, E., Serra, N., and Rhein, M. Variability of Labrador Sea Water transported through Flemish Pass during 1993-2013. Journal of Geophysical Research: Oceans, 120:5514-5533, 2015 .

Schoonover, J., Dewar, W., Wienders, N., Gula, J., Mcwilliams, J., Molemaker, J., Bates, S., Danabasoglu, G., and Yeager, S. North Atlantic barotropic vorticity balances in numerical models. Journal of Physical Oceanography, 1:289-303, 2015.

Schott, F., Stramma, L., and Fischer, J. Interaction of the North Atlantic Current with the deep Charlie Gibbs Fracture Zone throughflow. Geophysical Research Letters, 26:369-372, 1999.

Schott, F. A., Zantopp, R., Dengler, M., Fischer, J., and Wibaux, M. Circulation and deep-water export at the western exit of the subpolar North Atlantic. Journal of Physical Oceanography, 34:817-843, 2004.

Schott, F. A., Fischer, J., Dengler, M., and Zantopp, R. Variability of the Deep Western Boundary Current east of the Grand Banks. Geophysical Research Letters, 33:L21S07, 2006 . 
Send, U., Lankhorst, M., and Kanzow, T. Observation of decadal change in the Atlantic meridional overturning circulation using 10 years of continuous transport data. Geophysical Research Letters, 38:L24606, 2011.

Silverthorne, K. E. and Toole, J. M. Seasonal kinetic energy variability of near-inertial motions. Journal of Physical Oceanography, 39:1035-1049, 2009.

Smethie, W. M., Fine, R. A., Putzka, A., and Peter, E. Tracing the flow of North Atlantic Deep Water using chlorofluorocarbons. Journal of Geophysical Research, 105:14,297 14,323, 2000.

Smethie, W. M. J. Tracing the thermohaline circulation in the western North Atlantic using chlorofluorocarbons. Progress in Oceanography, 31:51-99, 1993.

Smethie, W. M. J. and Fine, R. A. Rates of North Atlantic Deep Water formation calculated from chlorofluorocarbon inventories. Deep Sea Research I, 48:189-215, 2001.

Smith, J. N., Smethie, W. M. J., Yashayaev, I., Curry, R., and Azetsu-Scott, K. Time series measurements of transient tracers and tracer-derived transport in the Deep Western Boundary Current between the Labrador Sea and the subtropical Atlantic Ocean at Line W. Journal of Geophysical Research: Oceans, 121, 2016.

Spall, M. A. Dynamics of the Gulf Stream/Deep Western Boundary Currrent crossover. Part 1: Entrainment and recirculation. Journal of Physical Oceanography, 26:2152-2168, 1996a.

Spall, M. A. Dynamics of the Gulf Stream/Deep Western Boundary Current crossover. Part 2: Low-frequency internal oscillations. Journal of Physical Oceanography, 26:2169-2182, 1996b.

Srokosz, M. A. and Bryden, H. L. Observing the Atlantic Meridional Overturning Circulation yields a decade of inevitable surprises. Science, 348:1255575, 2015.

Stommel, H. The westward intensification of wind-driven ocean currents. Trans Amer Geophys Union, 29:202-206, 1948.

Stommel, H. A survey of ocean current theory. Deep Sea Research (1953), 4:149-184, 1957.

Stommel, H., Arons, A. B., and Faller, A. J. Some Examples of Stationary Planetary Flow Patterns in Bounded Basins. Tellus, 10:179-187, 1958.

Stramma, L., Kieke, D., Rhein, M., Schott, F., Yashayaev, I., and Koltermann, K. P. Deep water changes at the western boundary of the subpolar North Atlantic during 1996 to 2001. Deep Sea Research I, 51:1033-1056, 2004.

Straneo, F. On the connection between dense water formation, overturning, and poleward heat transport in a convective basin. Journal of Physical Oceanography, 36:1822-1840, 2006 .

Sverdrup, H. U. Wind-driven currents in a baroclinic ocean; with application to the equatorial currents of the eastern Pacific. Proceedings of the National Academy of Sciences, 33: 318-326, 1947. 
Swallow, J. and Worthington, L. An observation of a deep countercurrent in the Western North Atlantic. Deep Sea Research (1953), 8:1-19, 1961.

Swift, J. H. The circulation of the Denmark Strait and Iceland-Scotland overflow waters in the North Atlantic. Deep Sea Research, 31:1339-1355, 1984.

Talley, L. D. and McCartney, M. S. Distribution and Circulation of Labrador Sea Water. Journal of Physical Oceanography, 12:1189-1205, 1982.

Thomas, M. D., De Boer, A. M., Johnson, H. L., and Stevens, D. P. Spatial and temporal scales of Sverdrup balance. Journal of Physical Oceanography, 44:2644-2660, 2014.

Thompson, J. D. and Schmitz, W. J. A limited-area model of the Gulf Stream: Design, initial experiments, and model-data intercomparison. Journal of Physical Oceanography, 19:791-813, 1989.

Thompson, R. O. R. Y. and Luyten, J. R. Evidence for bottom-trapped topographic Rossby waves from single moorings. Deep Sea Research, 23:629-635, 1976.

Toole, J. M., Curry, R. G., Joyce, T. M., McCartney, M., and Peña-Molino, B. Transport of the North Atlantic Deep Western Boundary Current about $39^{\circ} \mathrm{N}, 70^{\circ} \mathrm{W}: 2004-2008$. Deep Sea Research II, 58:1768-1780, 2011.

Vage, K., Pickart, R. S., Thierry, V., Reverdin, G., Lee, C. M., Petrie, B., Agnew, T. A., Wong, A., and Ribergaard, M. H. Surprising return of deep convection to the subpolar North Atlantic Ocean in winter 2007-2008. Nature Geoscience, 2:67-72, 2008.

Vallis, G. K. Atmospheric and Oceanic Fluid Dynamics. Cambridge University Press, 2006.

Van Aken, H. M. and De Boer, C. J. On the synoptic hydrography of intermediate and deep water masses in the Iceland Basin. Deep-Sea Research Part I, 42:165-189, 1995.

Van Sebille, E., Baringer, M. O., Johns, W. E., Meinen, C. S., Beal, L. M., De Jong, M. F., and Van Aken, H. M. Propagation pathways of classical Labrador Sea water from its source region to $26^{\circ}$ N. Journal of Geophysical Research: Oceans, 116:C12027, 2011.

Vellinga, M. and Wood, R. A. Impacts of thermohaline circulation shutdown in the twentyfirst century. Climatic Change, 91:43-63, 2008.

Waterman, S. and Jayne, S. R. Eddy-mean flow interactions in the along-stream development of a western boundary current jet: An idealized model study. Journal of Physical Oceanography, pages 682-707, 2011.

Waterman, S., Hogg, N. G., and Jayne, S. R. Eddy-Mean flow interaction in the Kuroshio extension region. Journal of Physical Oceanography, 41:1182-1208, 2011.

Waugh, D. W. and Hall, T. M. Propagation of tracer signals in boundary currents. Journal of Physical Oceanography, 35:1538-1552, 2005.

Worthington, L. V. On the North Atlantic circulation, volume 6. Johns Hopkins University Press, 1976.

Wunsch, C. The total meridional heat flux and its oceanic and atmospheric partition. Journal of Climate, 18:4374-4380, 2005. 
Wunsch, C. The decadal mean ocean circulation and Sverdrup balance. Journal of Marine Research, 69:417-434, 2011.

Wunsch, C. and Heimbach, P. Dynamically and kinematically consistent global ocean circulation and ice state estimates. In International Geophysics Series Volume 103: Ocean Circulation and Climate, pages 553-580. Academic Press, Elsevier, 2013a.

Wunsch, C. and Heimbach, P. Two decades of the atlantic meridional overturning circulation: Anatomy, variations, extremes, prediction, and overcoming its limitations. Journal of Climate, 26:7167-7186, 2013b.

Wunsch, C. and Roemmich, D. Is the North Atlantic in Sverdrup balance? Journal of Physical Oceanography, 15:1876-1880, 1985.

Wüst, G. Schichtung und Zirkulation des Atlantischen Ozeans. Die Stratosphaere. Wissenschaftliche Ergebnisse der Deutschen Atlantischen Expedition auf dem Forschungs- und Vermessungsschiff "Meteor" 1925 - 1927, 6, 1935.

Xu, X., Rhines, P. B., Chassignet, E. P., Schmitz, W. J., Jr, W. J. S., and Schmitz, W. J. Spreading of Denmark Strait overflow water in the western subpolar North Atlantic: Insights from eddy-resolving simulations with a passive tracer. Journal of Physical Oceanography, 45:2913-2932, 2015.

Yashayaev, I. Hydrographic changes in the Labrador Sea, 1960-2005. Progress in Oceanography, 73:242-276, 2007.

Yashayaev, I. and Loder, J. W. Enhanced production of Labrador Sea Water in 2008. Geophysical Research Letters, 36, 2009.

Yashayaev, I., van Aken, H. M., Holliday, N. P., and Bersch, M. Transformation of the Labrador Sea Water in the subpolar North Atlantic. Geophysical Research Letters, 34: L22605, 2007.

Yeager, S. Topographic coupling of the Atlantic overturning and gyre circulations. Journal of Physical Oceanography, 45:1258-1284, 2015.

$\mathrm{Yu}, \mathrm{L}$. and Jin, X. Buoy perspective of a high-resolution global ocean vector wind analysis constructed from passive radiometers and active scatterometers (1987 to present). Journal of Geophysical Research, 117:1-24, 2012.

Zhang, R. and Vallis, G. K. The role of bottom vortex stretching on the path of the North Atlantic western boundary current and on the northern recirculation gyre. Journal of Physical Oceanography, 37:2053-2080, 2007.

Zou, S. and Lozier, M. S. Breaking the linkage between Labrador Sea Water production and its advective export to the subtropical gyre. Journal of Physical Oceanography, 46: 2169-2182, 2016. 